- Aldivanced photemic sigunall processing

\title{
cannd
}

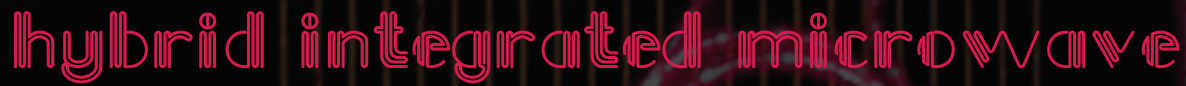
$C^{b} \cdot a^{2}$

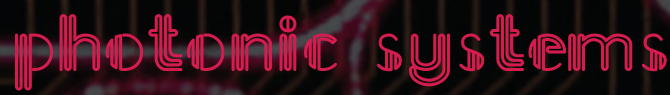

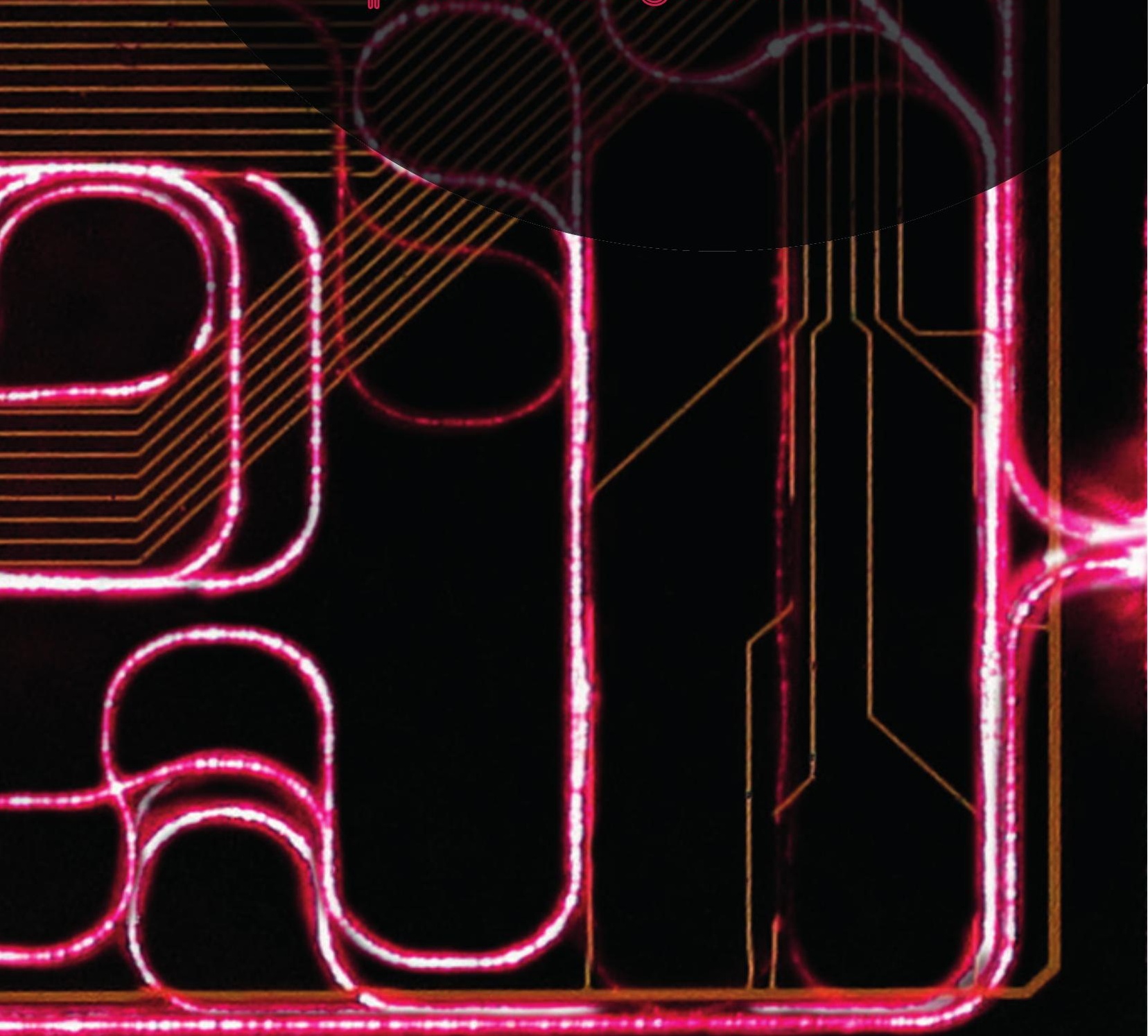




\section{ADVANCED PHOTONIC SIGNAL PROCESSING AND \\ HYBRID INTEGRATED MICROWAVE PHOTONIC SYSTEMS}

Caterina Taddei 


\title{
ADVANCED PHOTONIC SIGNAL PROCESSING AND \\ HYBRID INTEGRATED MICROWAVE PHOTONIC SYSTEMS
}

\author{
DISSERTATION
}

\author{
to obtain \\ the degree of doctor at the University of Twente, \\ on the authority of the rector magnificus, \\ prof. dr. T.T.M. Palstra \\ on account of the decision of the Doctorate Board, \\ to be publicly defended \\ on Friday the $7^{\text {th }}$ of December 2018 at 10:45
}

\section{by Caterina Taddei}

born on the $18^{\text {th }}$ of January 1984

in Castiglione del Lago (Perugia), Italy 
This dissertation has been approved by:

Supervisor: Prof. dr. K.-J. Boller

Co-supervisor: Dr. ir. C.G.H. Roeloffzen

Cover design: 8-ring resonators filter chip realized in $\mathrm{Si}_{3} \mathrm{~N}_{4} / \mathrm{SiO}_{2}$ waveguide technology

Printed by: Gildeprint

Lay-out: Caterina Taddei

ISBN: 978-94-6323-431-3

(C)2018 Caterina Taddei, The Netherlands. All rights reserved. No parts of this thesis may be reproduced, stored in a retrieval system or transmitted in any form or by any means without permission of the author.

The research presented in this thesis was carried out at the Laser Physics and Nonlinear Optics group, Department of Science and Technology, MESA+ Institute for Nanotechnology, University of Twente, P.O. Box 217, $7500 \mathrm{AE}$ Enschede, The Netherlands. This research is supported by IOP Photonic Devices program of Rijksdienst voor Ondernemend Nederland, part of the Netherlands Ministry of Economic Aairs (project number IPD12009) and partly supported by the Dutch Technology Foundation STW, which is part of the Netherlands Organization for Scientic Research (NWO). 


\section{Graduation committee:}

\section{Chairman and Secretary:}

Prof. dr. ir. J.W.M. Hilgenkamp

\section{Supervisor:}

Prof. dr. Klaus-J. Boller

\section{Co-supervisor:}

Dr. ir. Chris G.H. Roeloffzen

\section{Members:}

Prof. dr. Antonella Bogoni

Prof. dr. ir. Andreas Sthör

Prof. dr. ir. Frank Van Vliet

Prof. dr. Pepijn Pinkse

Dr. ir. Maurizio Burla

Dr. ir. David Marpaung
University of Twente, TNW

University of Twente, TNW

LioniX International

Scuola Superiore Sant' Anna, Pisa University of Duisburg-Essen

University of Twente, EEMCS/ICD University of Twente ETH Zürich Unversity of Twente, TNW 


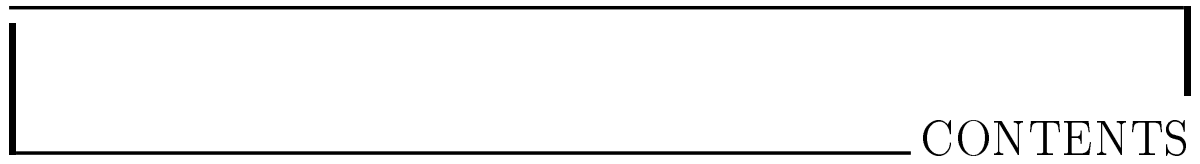

Summary $\quad$ xi

$\begin{array}{ll}\text { Nederlandse Samenvatting } & \text { xv }\end{array}$

1 Introduction $\quad 1$

1.1 Introduction . . . . . . . . . . . . . . 1

1.2 Outline of the thesis ............... 6

2 Performance metrics of an analog photonic link $\quad 7$

2.1 Fundamentals of analog photonic links . . . . . . . . . 7

2.1.1 Light generation .............. 8

2.1.2 Optical modulation .............. 9

2.1.3 Photodetection ................... 13

2.2 Analog photonic links and performance . . . . . . . . 15

2.2 .1 Link gain ................. 16

2.2.2 Noise in an analog photonic links . . . . . . . . 16

2.2.3 Non-linearities ................ 19

3 Modelling of microwave photonic filters 25

3.1 Introduction .......................... 25

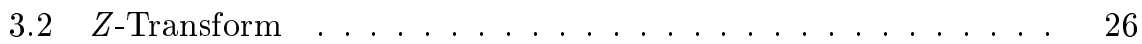


3.2.1 Normalized group delay . . . . . . . . . . . 28

3.3 Modelling of photonic building blocks and filters . . . . . . . 28

4 Antenna array theory $\quad 45$

4.1 Introduction . . . . . . . . . . . . . . 45

4.2 Radiation pattern of array antennas ............ 49

4.3 Phased array antennas . . . . . . . . . . . 51

4.4 Timed array antennas ................ 52

5 Coupled ring resonators waveguide filter $\mathbf{5 5}$

5.1 Introduction . . . . . . . . . . . . . . 55

5.2 Functional design of the coupled resonators optical waveguide filter ................... 56

5.3 Fabrication and demonstration of the coupled resonators waveguide filter . . . . . . . . . . . . . . 59

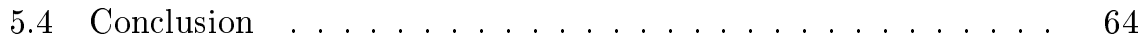

6 Hybrid integrated $4 \times 1$ receiver equipped with ring resonatorsbased optical beamforming network

6.1 Introduction . . . . . . . . . . . . . . 65

6.2 Hybrid integrated laser . . . . . . . . . . . . 66

6.3 Optical single-sideband modulation and single-sideband filter . $\quad 69$

6.4 Optical beamforming networks ............ 70

6.5 Photodetection ...................... 72

6.5.1 Balanced detection . . . . . . . . . . . 76

6.6 Functional design of the $4 \mathrm{x} 1$ optical beamforming network receiver 77

6.6.1 System requirements . . . . . . . . . 78

6.6.2 Design and measurements of the hybrid integrated laser 81

6.6.3 Design and measurement of optical sideband filter . . . 83

6.6.4 Design and measurements of the optical beamforming network .................. 88

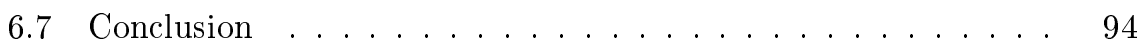

$7 \quad$ Hybrid integrated 1x4 transmitter equipped with switched delay lines-based optical beamforming network 
7.1 Introduction . . . . . . . . . . . . . . . . 97

7.2 OBFN based on switched delay line . . . . . . . . . . . 98

7.3 Functional design and layouts . . . . . . . . . . . . . . 98

7.3.1 Delay line chip . . . . . . . . . . . . . 100

7.3.2 Modulator and photodiodes chips . . . . . . . 101

7.3 .3 InP gain chip . . . . . . . . . . . . . . . . . . 101

7.4 Fabrication and measurements of the hybrid integrated microwave photonic system . . . . . . . . . . . 102

7.4 .1 Laser ...................... 104

7.4 .2 Link gain ................... 104

7.4 .3 Delay generation ............... 107

7.4.4 Modulators ................. 109

7.4.5 Noise and non-linear distorsions . . . . . . . . . . . . 111

7.5 Discussion and conclusion ............... 114

8 Conclusion and Outlook $\quad 117$

8.1 Conclusion ..................... 117

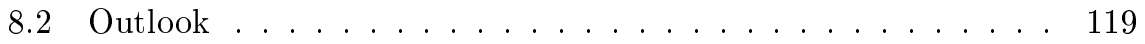

$\begin{array}{lr}\text { Bibliography } & \mathbf{1 2 1}\end{array}$

$\begin{array}{lr}\text { Publications } & 127\end{array}$

$\begin{array}{ll}\text { Acronyms } & \mathbf{1 3 1}\end{array}$

$\begin{array}{ll}\text { Acknowledgements } & \mathbf{1 3 5}\end{array}$ 

Ai miei genitori

Enrica e Antonio 

In the past ten years, the interdisciplinary research field known as "microwave photonics" (MWP) has attracted considerable interest in scientific and industrial communities. In brief, microwave photonics explores and develops methods and technologies to generate, process and distribute microwaves, millimeter waves and terahertz radiation via a photonic approach, i.e. in the optical domain. The main motivation for this approach is that systems based on microwave photonic technology can benefit from several advantages that are inherent to optical systems, such as high speed, low and frequency-independent propagation loss and reduced electromagnetic interference, applicable across the entire microwave (tens of $\mathrm{GHz}$ ) and millimeter range (hundreds of $\mathrm{GHz}$ ) with potentially smaller size and lower weight than the well-established electronic systems.

Microwave photonic technology is extremely flexible and therefore its possible applications are very diverse, ranging from broadband wireless networks, to radar and satellite communications, sensing, to ranging in autonomous traffic. However, although there is an indisputable and significant potential of microwave photonics, it has not yet been applied in real contexts. The main reason is that microwave photonics so far had to rely mostly on discrete components, which render according microwave photonic systems bulky, unstable and fragile. In order to overcome these limitations, while still taking advantage of the named, great opportunities, research and technology have begun targeting integration of microwave photonic systems, with the goal to enable the processing of microwave and millimeter waves via photonic chips.

In this thesis, we investigate how integrated photonic technologies can be exploited to realize integrated microwave photonic systems. In particular, our interest has been the design, fabrication, integration and testing of state-ofthe-art integrated microwave photonic optical beamforming networks for $\mathrm{K}_{\mathrm{u}^{-}}$, 
and $\mathrm{K}_{\mathrm{a}}$-band satellite communications, based on hybrid integration of III-V semiconductor (indium phosphide) components with low-loss and high index contrast dielectric waveguide circuits (silicon nitride).

After the introduction, Chapter 2 presents the basic working principles and properties of analogue photonic links, also called microwave photonic link. The parameters discussed form the basis to describe quantitatively and qualitatively the performance of the hybrid integrated microwave photonic demonstrators presented in this thesis.

For a quantitative description of most essential photonic building blocks, Chapter 3 describes the modelling of microwave photonic filters via the $\mathrm{z}$ transform, specifically integrated Mach-Zehnder interferometers, microring resonators, Mach-Zehnder interferometers loaded with ring resonators, and other combinations.

To present also an overview on the design of the beamforming system beyond its photonic core components, Chapter 4 presents the essential properties of the microwave array antennas that are to be photonically controlled. In particular, the difference between array antennas based on phase shifters and array antennas based on true-time delays is pointed out, since the latter option enables to provide a squint-free transmission or reception. The basic delays units realized and presented here are based on switched optical delay lines and tunable optical ring resonators.

After these section that provide merely theoretical overview and details, a first complex reconfigurable photonic filter realized during the course of the project, based on silicon nitride, is presented in Chapter 5. The filter consists of a combination of eight mutually coupled ring resonators in order to have a highly selective on-chip passband filter with a bandwidth of $72 \mathrm{MHz}$ and an out-of-band rejection of at least $51 \mathrm{~dB}$. Such a filter can be potentially used in a satellite payload to increase the capacity and enhance the flexibility of high throughput satellites.

Chapter 6 and Chapter 7 presents hybrid integrated microwave photonic systems forming again the state of the art. The processing cores of these demonstrators are formed by true-time delay optical beam forming networks. Both the demonstrators are fully integrated, meaning that the inputs and outputs are signals in the microwave domain, while the processing is done in the optical domain. Both systems are equipped with an internal hybrid laser, consisting of indium phosphide semiconductor optical amplifiers (SOAs) coupled to an external cavity arm fabricated on the silicon nitride platform, to provide an on-chip optical carrier.

Several different modulation schemes were tested to explore the efficiencies that can be reached. The demonstrator presented in Chapter 6 is based on phase modulation combined with optical filtering to achieve single-sideband modulation, while the demonstrator in Chapter 7 is based on intensity modulation to achieve double-sideband modulation. Both systems are based on 
a true-time delay optical beamforming network, though the basic delay units are different. Namely, the unit described in Chapter 6 is based on a number of cascaded ring resonators, in order to have continuously tunable delay, while the unit delay given in Chapter 7 is based on switched delay lines, which provides a stepwise variable true-time delay. Both systems comprise hybrid integrated photodetectors for optical-to-electrical conversion.

Together with the testing of the different functionalities, such as filters, optical beamforming networks, splitters and combiners, which were all successfully demonstrated, the other main goal of the research was to explore the viability of the concept of hybrid integration, in particular, integration of indium phosphide and silicon nitride platforms. Chapter 7 shows a fully successful hybrid integration, thereby having reached a highly important milestone in integrated microwave photonics. This demonstration can be considered a crucial and fundamental benchmark for the next generation of hybrid integrated photonic systems. 
In de afgelopen tien jaar heeft het interdisciplinaire onderzoeksveld, dat microgolf fotonica wordt genoemd, veel aandacht gekregen vanuit de wetenschap en industrie. Hierbij ligt de focus met name op de mogelijkheid om mircogolven, millimetergolven en terahertz signalen in het optische domein op te wekken, te verwerken, te analyseren en te distribueren. Systemen die op microgolf fotonica technologie gebaseerd zijn bieden verschillende intrinsieke voordelen vanuit de optica, namelijk: hoge transmissie snelheden en grote bandbreedte, een verlaagde gevoeligheid voor elektromagnetische interferentie, een breed werkbereid over de volledige microgolf (tientallen $\mathrm{GHz}$ ) en millimetergolf (honderden $\mathrm{GHz}$ ) spectra, lage en frequentie-onafhankelijke propagatieverliezen en ten slotte kleine afmetingen en een laag gewicht.

Microgolf fotonica technologie is toepasbaar in een groot, zeer divers, toepassingsgebied dat onder andere de velden van breedband draadloze netwerken, radar/satelliet communicatie, sensoren, en afstandmetingen omspant zoals in autonome verkeerssystemen. Echter, hoewel de potentie van microgolf fotonica niet te ontkennen valt, is het nog niet toegepast in een commercieel product. De voornaamste reden is dat bij huidige implementaties van microgolf fotonica voornamelijk discrete componenten gebruikt worden, waardoor de systemen groot, zwaar, instabiel en onbetrouwbaar worden. Om deze tekortkomingen te overkomen, terwijl de bovengemoede voordelen behouden blijven, richt het veld van geïntegreerde microgolf fotonica zich op het creëren van geïntegreerde fotonische systemen die in staat zijn om micro- en millimetergolf signalen te verwerken door middel van fotonische chips.

In dit proefschrift beschrijf ik mijn onderzoek naar hoe geïntegreerde fotonische technologie toegepast kan worden in geïntegreerde microgolf fotonische systemen. Hierbij heb ik me vooral gericht op het ontwerp, de fabricage, de integratie en het testen van innovatief geïntegreerde netwerken voor microgolf 
fotonische optische bundelvorming voor $\mathrm{K}_{\mathrm{u}^{-}}$, en $\mathrm{K}_{\mathrm{a}}$-band satellietcommunicatie op basis van de hybride integratie van III-V halfgeleidermaterialen op siliciumnitride.

Er wordt eerst een introductie gegeven over de belangrijkste prestatieindicatoren voor een analoog optische verbinding, ook wel microgolf fotonische verbinding genoemd. De geïntroduceerde parameters worden later gebruikt om de prestaties van de microgolf fotonische modules zowel quantitatief als kwalitatief te beschrijven.

Daarna wordt een methode voor het modelleren van microgolf fotonische filters, op basis van de $z$-transformatie, beschreven samen met de modellen van de meest gangbare fotonische bouwblokken - waaronder Mach-Zehnder interferometers, ringresonatoren, Mach-Zehnder interferometers die gekoppeld zijn met ringresonatoren en andere combinaties.

De belangrijkste eigenschappen van een microgolf array antenne worden beschreven in hoofdstuck 4. Er wordt specifiek aandacht besteed aan het verschil tussen antenne-arrays die gebaseerd zijn op faseverschuifers en antennearrays die gebaseerd zijn op de ware tijdsvertragingen, omdat de laatste van deze twee de garantie van een kwispel vrij antenne-array biedt. De tijdsvertragingseenheden die gebruikt worden in dit werk zijn gebaseerd op geschakelde vertragings lijnen of optische ringresonatoren.

$\mathrm{Na}$ de introductie van de theoretische aspecten, wordt in hoofdstuk 5 het eerste complexe configureerbare fotonische filter in siliciumnitride beschreven. Het filter bestaat uit een combinatie van acht gekoppelde ringresonatoren om tot een hoog-selectieve on-chip band-doorlaat filter met een bandbreedte van $72 \mathrm{MHz}$ en een onderdrukking van ten minste $51 \mathrm{~dB}$ te komen. Het filter is ontworpen om toegepast te worden in satelliettechniek om daar de capaciteit en flexibiliteit van satellieten te verhogen.

In hoofdstuk 6 en 7 worden de innovatief hybride geïntegreerde microgolf fotonische systemen gepresenteerd, waar de verwerkingskern gebaseerd is op bundelvormingsnetwerken op basis van ware tijdvertraging. Beide modules zijn volledig geïntegreerd, wat betekent dat de in- en uitgangen in het microgolf domein liggen terwijl de signaalverwerking in het optische domein gedaan wordt. Om dit te bereiken zijn beide systemen uitgerust met interne hybride lasers - welke bestaan uit een indiumfosfide diode versterker die gekoppeld is aan een externe holtearm die op het siliciumnitride platform is bevestigd - die de optische draaggolf verzorgt.

Verschillende modulatieschemas zijn getest om de efficiëntie te evalueren. De module die in hoofdstuk 6 wordt beschreven is gebaseerd op fasemodulatie gecombineerd met filteren, om een enkelzijband modulatie te bereiken, terwijl de module uit hoofdstuk 7 gebaseerd is op intensiteitsmodulatie met als doel een dubbelzijband modulatie te bereiken. Beide systemen zijn op optische bundelvormingsnetwerken gebaseerd op ware tijdvertragingen, echter de basis vertragingseenheden voor beide systemen verschillend. De basis vertragingseen- 
heid van het systeem in hoofdstuk 6 is gebaseerd op een aantal geschakelde ringresonatoren om een continue varieerbare delay te bereiken, terwijl die van het systeem in hoofdstuk 7 gebaseerd is op geschakelde vertraginslijnen, welke stapsgewijze tijdsvertragingen oplevert. Beide systemen hebben hybride geïntegreerde fotodetectoren voor de optisch-naar-electrisch conversie.

Samen met het testen van de verschillende functionaliteiten, zoals filters, optische bundelvormingsnetwerken, verdelers en combineerders - welke allemaal succesvol gedemonstreerd zijn -, was het belangrijkste doel van dit onderzoek het verifieren van het hybride integratieconcept van met name indiumfosfide en siliciumnitride optische golfgeleiders. In hoofdstuk 7 wordt een volledig succesvolle hybride integratie besproken. Dit is een mijlpaal voor het veld van geïntegreerde microgolf fotonica en kan bovenal beschouwd worden als een benchmark voor de volgende generatie van hybride geïntegreerde fotonische systemen. 


\section{CHAPTER 1}

INTRODUCTION

\subsection{Introduction}

Human beings have always felt the need to communicate, to exchange pieces of information. The further the distance, the more creative the solutions for communicating have been. Smoke signals during the day whereas fires during the night were the tools used to set a communication link in the olden days. Nowadays, tools are definitely different but the aim of a communication link remains the same.

A communication network, the way it is intended in the modern time, performs two main functionalities: the transfer of information from one point in the network to another and the dynamic assignment of the available transmission channels, upon users connection requests. At first those two functionalities have been performed using analog technologies, relying mostly on copper cables as transmission media. Later on, with the digital revolution [1], i.e., the adoption and proliferation of digital technologies, which began from the late 1950s to the late 1970s, those activities became faster and more efficient and, as a consequence, the transmission networks became capable of transfering a higher amount of information. In the 1970s and 1980s other important technological achievements allowed to develop even further the world of telecommunications. The first geostationary satellites were launched extending the distances that telecommunication links could cover. However, it is only with the advent of the "wireless revolution", which began in the 1980s, that the way of communicating, as it is known now, took off.

Starting in the 1980s, the first generation (1G) of wireless communications, relying on the microwave and radio frequency $(\mathrm{RF})$ domain, had started to 
spread and changed the life of almost everyone in the world. Each next generation of wireless communications added new features which would change the way of living. This journey through evolution has been, at the same time, cause and consequence of an incredible technological growth, in order to find the "perfect ingredients" for what is going to be the new paradigm for the next generation of communications, the fifth generation, 5G. The need of developing and defining a fifth generation of networks is due to the explosive increase in demand for wireless broadband services needing faster, higher-capacity and ultra-reliable networks. A complete standardization of the $5 \mathrm{G}$ generation of wireless communications does not exist yet, because standards usually tend to be defined quite late in the evolution of a new generation. Many big players in the telecommunication market are still evaluating which aspects of a telecommunication link have to be addressed and enhanced and how to do so. As an example, some areas, such as network architectures, coding schemes, multiplexing schemes, antennas and control of antennas are at the focus of the current research to address the $5 \mathrm{G}$ performance requirements. The existing electronic solutions have to be extended in order to fulfill the challenging specifications.

Parallel to the evolution of wireless communications, the field of optical communications went through its own evolution, especially from the 1970s, when high-quality, low-loss optical fibers could be manufactured at a speed of over 50 meters per second [2], together with the availability of semiconductor lasers and fast photodetectors. Since the loss in optical fibers is much lower and the available bandwidth is much wider than in electrical signal transport, optical telecommunications became widespread. Primarily, the interest was driven by the possibility to use optical fibers for transporting digital information. Only afterward, it was pointed out that optical fibers could be used as well for the transmission of analogue microwave and RF signals. An increased awareness of the potential of what could be done in the optical domain made optical fibers and optical techniques attractive not only as a mere transmission medium, but also as signal processing platform.

The novel field that brings together the worlds of radio-frequency engineering and optics is now being called microwave photonics (MWP) [3], [4]. Processing data with methods and technologies from MWP becomes particularly suitable for high-performance broadband communication systems, because of its most attractive features of low loss, low weight, large bandwidth, and immunity to electromagnetic interference.

Despite the high potential of the MWP field, so far it did not spread in real-life applications. The main cause is the high cost, the bulkiness, complexity and power consumption in the presently available systems [5]. Another important limitation is an often insufficient reliability of the system because of the interconnection between discrete components. In addition to being a problem itself in terms of size, using discrete components entails timing and phase instabilities, and the system's sturdiness can be compromised through environmental perturbations, such as temperature or mechanical variations. 
Table 1.1: Comparison among common photonic platforms

\begin{tabular}{||c|c|c|c||}
\hline Platform & InP & SOI & $\mathbf{S i}_{3} \mathbf{N}_{\mathbf{4}} / \mathbf{S i O}_{\mathbf{2}} \mathbf{P L C s}$ \\
\hline \hline Index Contrast & $5-10 \%$ & $40-45 \%$ & $20-30 \%$ \\
\hline Attenuation $(\mathrm{dB} / \mathrm{cm})$ & 2.5 & $<2$ & 0.1 \\
\hline Light Generation & $\sqrt{ }$ & $\boldsymbol{X}$ & $\boldsymbol{X}$ \\
\hline Modulation & $\sqrt{ }$ & $\boldsymbol{X}$ & $\boldsymbol{X}$ \\
\hline Amplification & $\sqrt{ }$ & $\boldsymbol{X}$ & $\boldsymbol{X}$ \\
\hline Detection & $\sqrt{ }$ & $\boldsymbol{X}$ &
\end{tabular}

Therefore, to overcome the aforementioned limitations and still take advantage of the incredible fetaures provided by MWP, the common consensus that has emerged is that MWP systems need to exploit integrated photonic technologies to develop their great impact. In fact, it is not surprising that the "key" lies in the integration. This process resembles a similar trajectory as with the electronic integrated circuits, when in 1958 Jack Kilby tried to solve the problem known as "The Tyranny of Numbers" proposing the concept of integration into monolithic electronic systems [6]. Just like the idea of Kilby, the aim of the novel field of integrated microwave photonics (IMWP) is to incorporate different MWP components and sub-systems in monolithic or hybrid integrated photonic devices. There is a difference, though. In IMWP it is not possible to be bound to a monolithic approach because there is no single platform that addresses efficiently all the IMWP performance requirements, without sacrificing overall system performance [7]. It has turned out that only three platforms are mainly used in IMWP systems. These are Indium Phosphide (InP), silicon-oninsulator (SOI) and planar light wave circuits (PLCs) based on silicon nitride $\left(\mathrm{Si}_{3} \mathrm{~N}_{4}\right)$. Each of these exhibits its strength in performing one or more specific tasks best. A short comparison among the three technologies for some key parameters and functionalities is given in Table 1.1 [5].

InP is the only material that can be potentially used with a monolithic approach since light generation, modulation, detection and processing can be performed. However, the attenuation which the light experiences in propagation through InP waveguides is relatively high when compared for instance to the waveguides made of silicon nitride.

SOI and silicon nitride are both silicon-based. The first platform uses silicon as core of the waveguides, while the second one is based on silicon nitride as core surrounded by silicon oxide. Both platforms are optically passive, in the sense that functionalities, such as light generation, modulation, and detection cannot be efficiently performed. In SOI light is more confined (index contrast 40-45\%) and the SOI chips can thus have a smaller footprint. However, SOI suffers from higher loss compared to PLCs using $\mathrm{Si}_{3} \mathrm{~N}_{4} / \mathrm{SiO}_{2}$. Moreover, the power which can be handled in SOI waveguides has an upper limit, wich is in the order of tens of milli-Watts. 
PLCs based on $\mathrm{Si}_{3} \mathrm{~N}_{4} / \mathrm{SiO}_{2}$ are characterized by extremely low propagation loss, as low as $0.1 \mathrm{~dB} / \mathrm{cm}[8]$, and in straight waveguides with weak guiding even less than $0.001 \mathrm{~dB} / \mathrm{cm}[9]$. These properties make this platform particularly suitable when, for instance, long delay lines are needed. Moreover, it is possible to shape (taper) the waveguides in order to have an optimum chip-to-chip coupling to waveguides made of other materials, such as InP, to add active functionalities. Another aspect to take into account is the capability of siliconnitride/silicon-oxide to handle relatively high power, in the order of Watts, which is particularly important for MWP applications.

An attempt of monolithic integration of an IMWP filter in the InP platform is presented in [10]. Such a system is indeed very compact, but suffers from high losses and severe RF interference, due to the close proximity of active components within a compact area.

An alternative approach is heterogeneous integration of $\mathrm{InP}$ with SOI. Here, a monolithic silicon photonic process is used as a base and active devices based on III-V materials are wafer-bound to it, i.e., the alignment with the SOI waveguides is achieved lithographically [11].

The third possible approach is hybrid integration, meaning that two or more separate chips are brought together after fabrication to produce a multichip system. This approach allows to combine rather different material platforms, thereby taking better advantage of the individual strengths. It is important to point out that the hybrid integrated systems are playing a big role in the field of IMWP and that, at this point, assembling and packaging processes are governing the further development of the hybrid integration concept. The importance of the assembling and packaging is confirmed also by the joint effort of academic groups, industry and international organizations with an excellent know-how in the field of IMWP. Sharing, on a global scale, the expertise to develop and consolidate the according IMWP ecosystem is a must. Recently, within the COST framework [12], the European Network for High Performance Integrated Microwave Photonics (EUIMWP) Action has been established to support the coordination among the relevant IMWP communities around Europe. Another noteworthy European activity is the Photonic Hybrid Assembly Through Flexible Waveguides (PHASTFlex) project [13], whose aim is oriented towards the development of a fully automated and reliable assembly technology for the next generation of hybrid integrated photonic systems.

Figure 1.1 shows an example of how a processing core based on IMWP technologies can be part of a telecommunication front-end. With regard to its function, an IMWP system can be seen as a black box where all inputs and outputs are signals in the microwave or RF regime. This means that the according wavelengths are in the order of centimeters, while internally the processing is done in the optical domain, where the wavelengths are in the order of a micrometer. Typical components of such IMWP systems are lasers, modulators, photodetectors and also passive photonic components such as waveguide 
couplers, splitters and delay lines.

Integrated microwave photonic systems are highly promising candidates to be part of the new generation $(5 \mathrm{G})$ of wireless and satellite communications, to enhance the performance of communication links, as will be described in the next chapters. In this thesis, in order to explore the feasibility of hy-

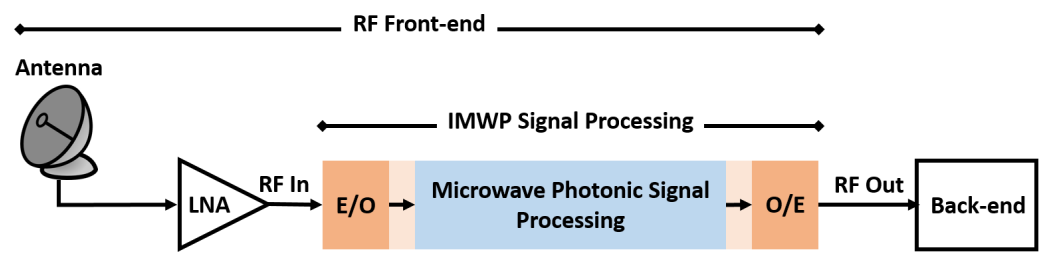

Figure 1.1: Front-end based on IMWP processing core

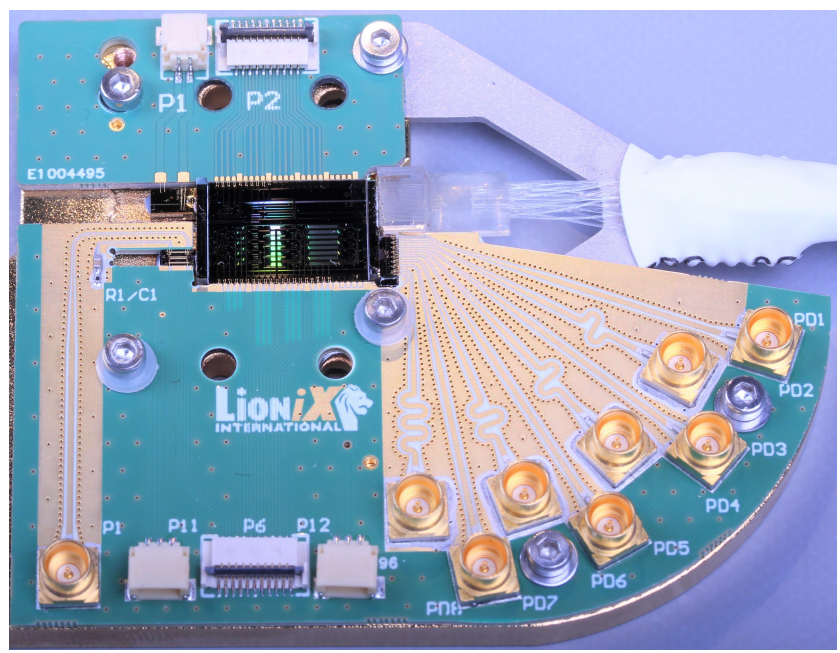

Figure 1.2: Photograph of a state-of-art hybrid IMWP system as investigated in chapter 6 of this thesis

brid IMWP systems, we have fabricated and experimentally demonstrated two IMWP multi-chip systems equipped with the most advanced photonic building blocks for complex signal processing. A photo of the most recent hybrid IMWP system is shown in Figure 1.2, and a more detailed description is given in Chapter 7.

Among the other approaches and waveguides platforms that have been previously presented for building an IMWP system for telecom applications, we have chosen the hybrid approach, by combining InP chips for active functionalities and PLCs based on $\mathrm{Si}_{3} \mathrm{~N}_{4} / \mathrm{SiO}_{2}$ for passive signal processing in the optical domain. The main motivation for this choice is that both these waveguide 
platforms have gained an appreciable maturity, i.e., that the fabrication of even rather complex circuits is comparably reliable and reproducible. Furthermore, the signal processing on $\mathrm{Si}_{3} \mathrm{~N}_{4} / \mathrm{SiO}_{2}$ guarantees ultra-low propagation loss, such as $0.1 \mathrm{~dB} / \mathrm{cm}$ for asymmetric double-stripe, i.e., double-core waveguide cross sections, and it is therefore capable to provide record-long waveguides configured in complex architectures in a compact chip. Such $\mathrm{Si}_{3} \mathrm{~N}_{4} / \mathrm{SiO}_{2}$ chips contain optical elements that can be controlled externally by thermo-optic control and recently also piezo-control [14], making the chip programmable and reconfigurable.

\subsection{Outline of the thesis}

After this introduction, the thesis begins in Chapter 2 with a review of the relevant theoretical concepts which have been used to design the fabricated IMWP systems. First the concept of an analog photonic link is addressed, which is the basic type of link within an MWP system, and we present the metrics commonly used to describe its performance. Chapter 3 is focused on the modelling of the basic building blocks that are present in the $\mathrm{Si}_{3} \mathrm{~N}_{4} / \mathrm{SiO}_{2}$ chips and that can be combined to achieve complex functionalities. Chapter 4 gives a review of the basic concepts of array antennas, since these can be considered the "natural environment" for the IMWP systems investigated here. Chapter 5 introduces and analyses an $8^{\text {th }}$-order MWP filter fabricated on the $\mathrm{Si}_{3} \mathrm{~N}_{4} / \mathrm{SiO}_{2}$ waveguide platform, demonstrating the central advantage of ultra-low loss in the silicon nitride platform. Chapter 6 and Chapter 7 describe the system overview, the functional design, the system integration and test of two stateof-the-art IMWP systems, whose aim is to be used as processing core of an array antenna. In the end, the thesis provides conclusions and recommendations for future developments and optimization of IMWP systems. 
CHAPTER 2

PERFORMANCE METRICS OF AN ANALOG PHOTONIC LINK

\subsection{Fundamentals of analog photonic links}

This chapter introduces the concept of an analog photonic link (APL), which is at the core of the emerging field of MWP, and we introduce the main constituting building blocks. After a description of the main components, the most relevant figures of merit of an APL will be described.

In its most basic form, an APL, as shown in Figure 2.1, consists of a laser, a modulation device and a photodetector, interconnected with, e.g., an optical fiber, in order to convey an electrical signal over an optical carrier, transfer information through optical fibers and convert back the signal from optical domain to electrical domain. Ultimately, an APL can be seen as a blackbox, where inputs and outputs are in the microwave domain, while instead

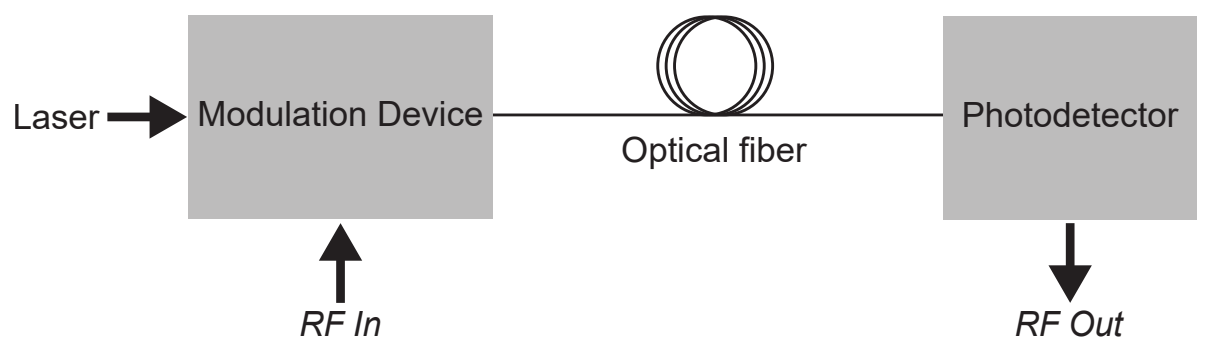

Figure 2.1: Schematic for an analog photonic link 
Performance metrics of an analog photonic link

any processing, which can be transfer or manipulation of the amplitude or the phase of signals, is happening in the optical domain.

In this section we will analyze the basic elements of the APL and their available structures, to have an overview of what is needed in and what can be achieved by an APL.

\subsubsection{Light generation}

There is a variety of lasers, that can be utilized for MWP applications. Ranging from semiconductor lasers to solid-state lasers, each kind of laser has its specific properties as advantages. However, in the MWP context, semiconductor lasers are preferred over the solid state ones, because of their property of generating light directly from an injected current, while in the second case light has to be injected to generate light (optical pumping of the solid state laser). Focusing on the semiconductor lasers, once again, there are different possibilities, such as distributed-feedback (DFB) lasers, distributed Bragg reflector (DBR) lasers, vertical-cavity surface-emitting lasers (VCSELs), and external cavity semiconductor lasers. Among the other kinds, the DFB lasers are the more commonly used in MWP systems. In a DFB laser the active region of the device is structured as a diffraction grating, that acts as the wavelength selective feedback element, in order to obtain single-frequency emission. The gain is based on a semiconductor junction, and the wavelength ranges that can be covered match the optical communication wavelengths, specifically, the O-band (1260 nm - $1360 \mathrm{~nm})$, E-band (1360 nm - $1460 \mathrm{~nm})$, S-band (1460 nm - $1530 \mathrm{~nm}$ ), C-band (1530 nm - $1565 \mathrm{~nm}$ ) and the L-band (1565 nm - 1625 $\mathrm{nm})$. In the past years, however, there has been a growing interest towards the external cavity semiconductor lasers. Because of the opportunity to integrate them in more complex systems, external cavity semiconductor lasers seems to be particularly promising as sources for IMWP, as discussed in Chapter 6 and Chapter 7 .

The output of the laser is used as optical carrier in an MWP system and regardless the type of employed laser, the optical carrier should have high power, low intensity noise and a small spatial linewidth. As it will be described through the next chapters, parameters such as output power, intensity noise and linewidth of the laser strongly influence the RF performance of the entire MWP system. In general, low intensity fluctuations are highly desired since photodetection converts intensity fluctuations into RF photocurrent fluctuations, which can distort the RF information to be transmitted or processed. The standard parameter that quantifies how stable the laser output power is vs. time, is the relative intensity noise (RIN). This parameter is defined as

$$
R I N=\frac{\left\langle\delta P_{o p t}(t)^{2}\right\rangle}{P_{0}^{2}},
$$

where $\delta P_{o p t}(t)$ is the optical intensity fluctuations, the \langle\rangle denotes the time 
average, and $P_{0}$ is the average optical power. It is common to express the RIN in $\mathrm{dB} / \mathrm{Hz}$ as

$$
R I N_{d B}=10 \log _{10}(R I N)[d B / H z] .
$$

Nevertheless, also phase-noise is an important parameter in MWP applications, especially, if information is encoded in the phase of the optical carrier or whenever one makes use of conversion between phase and intensity, such as in any interferometric effect.

In this thesis, for the theoretical description, the optical carrier field is taken to be a continuous wave $(\mathrm{CW})$ and is written as the time-varying complex valued quantity

$$
E_{i}(t)=E_{0}(t) e^{j\left(\omega_{0} t+\phi_{0}(t)\right)} \doteq \gamma \sqrt{2 P_{i}} e^{j\left(\omega_{0} t+\phi_{0}(t)\right)},
$$

where $\gamma$ is a constant relating the optical field and the average optical power such that $P_{i}=\left(E_{i}(t) \cdot E_{i}(t)^{*}\right) /\left(2 \gamma^{2}\right) . E_{0}(t), \omega_{0}$ and $\phi_{0}(t)$ are the amplitude, angular frequency and phase of the optical carrier, respectively.

\subsubsection{Optical modulation}

The RF information has to be up-converted from $\mathrm{MHz} / \mathrm{GHz}$ to hundreds of $\mathrm{THz}$ to be processed in optical domain. The up-conversion is obtained by the modulation of the light beam of the optical carrier by the RF signals which carry the information. The information can be encoded in different parameters of the optical carrier, such as for example the intensity, frequency, phase or polarization. Depending on which parameter is used as information-conveyor, modulators may be categorized into intensity modulators, phase modulators, polarization modulators and others. In this work, we focus on mainly two kinds of modulators, namely intensity modulators and phase modulators.

Often the easiest way to obtain modulation of the intensity of the optical carrier seems to be via direct modulation of the laser output power, by modulating its injection current. This sort of modulation is called direct modulation. However, despite the apparent practicality of such approach, there is a major downside, which is related to the strong frequency chirp caused by such modulation, that is the time dependence of the laser instantaneous frequency, $\omega_{0}(t)$. Having a chirped-carrier is undesired since it would have a spectral broadening with respect to a chirp-free optical carrier, which may negatively influence the RF performances of the MWP system. As it will be described in the next chapters, the modulated carrier will be processed by filters and having a spread spectrum because of the chirped-carrier would lead to the presence of unwanted frequency components at the photodetection.

As opposed to the direct modulation, external modulation is performed by an independent device, the external light modulator. In the simplest case, if the optical phase is to be modulated, one uses an external electro-optic waveguide modulator, whose refractive index changes according to the applied RF 


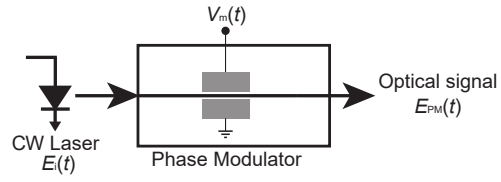

(a)

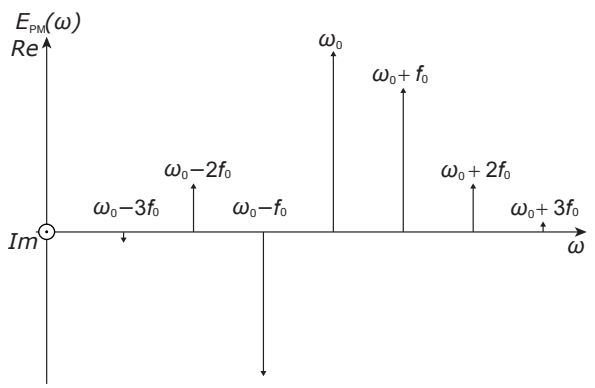

(b)

Figure 2.2: (a) Schematic for phase modulated optical carrier, (b) Phasor diagram for a phase modulator driven by a single-tone with a peak amplitude of $0.4 V_{\pi}$, an optical carrier frequency $\omega_{0}$ and modulation with an RF frequency $f_{0}$. The real-imaginary plane is normal to the page, with the positive imaginary axes coming out from the page.

field when made of suitable material. Instead, when the intensity or amplitude of the optical carrier is used to encode information, typically an interferometric approach is used to convert phase modulation into amplitude modulation (e.g., a Mach-Zehnder interferometer (MZI)). Intensity modulation can also be achieved by using another waveguide-based modulator, the electro-absorption modulator. In this case the applied RF field changes the absorption spectrum. In the next sections, we will focus on phase modulation and intensity modulation obtained by using electro-optic waveguide modulators since those are the types of modulations used in Chapter 6 and Chapter 7, respectively.

\subsubsection{Phase modulation}

In this section, phase modulation will be presented. Using phase modulation is convenient, compared to intensity modulation, since a constant amplitude phase-modulated signal is less susceptible to optical or electronic non-linearities in subsequent system components. Furthermore, there is usually no need to bias the modulator, as instead is required in the case of intensity modulation obtained using a Mach-Zehnder modulator (MZM) (see section 2.1.2.2). In order to illustrate how phase modulation modifies the optical spectrum of the carrier, in the following, we recall the calculation of the so-called single-tone response, where the RF input is a single-frequency (single-tone) oscillation,

$$
V_{m}(t)=V_{R F} \sin \left(2 \pi f_{R F} t\right)=V_{R F} \sin \left(\omega_{R F} t\right) .
$$

Here $V_{R F}$ is the peak amplitude of the RF modulating signal and $\omega_{R F}$ is the modulation angular frequency. A typical schematic for such modulation is depicted in Figure 2.2(a). The optical field at the output of the phase modulator 


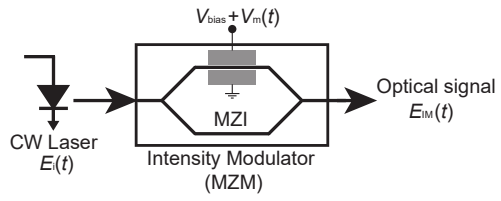

(a)

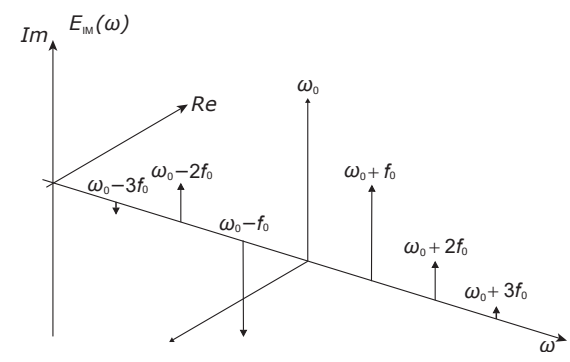

(b)

Figure 2.3: a) Schematic for intensity modulation of an optical carrier, b) Phasor diagram for an intensity modulator biased at quadrature point, driven by a single-tone with a peak amplitude of $0.4 V_{\pi, R F}$, an optical carrier frequency $\omega_{0}$ and modulation with an RF frequency $f_{0}$.

is then obtained as

$$
E_{P M}(t)=\beta e^{j\left(\omega_{0} t+\frac{\pi V_{R F}}{V_{\pi, R F}} \sin \left(\omega_{R F} t\right)\right)},
$$

where $\beta=\left(\gamma \sqrt{2 P_{i} / L_{P M, l i n}}\right), L_{P M, \text { lin }}$ is the power insertion loss of the modulator expressed on a linear scale and $V_{\pi, R F}$ is the frequency-dependent voltage required to produce a $\pi$ phase shift, known as half-wave voltage. The latter is one of the most important parameters of a modulator, which influences the overall RF performance of the APL, as we will discuss later in the next chapters. According to the Jacobi-Anger expansion, the modulated optical field contains an infinite number of side-frequencies and can be expressed as follows

$$
E_{P M}(t)=\beta \sum_{n=-\infty}^{\infty} J_{n}\left(\frac{\pi V_{R F}}{V_{\pi, R F}}\right) e^{j\left(\omega_{0} t+n \omega_{R F} t\right)} .
$$

Here, $J_{n}$ denotes the $n$-th Bessel function of the first kind. Figure 2.2(b) shows the typical shape of the spectrum described by (2.6).

\subsubsection{Intensity modulation}

The most common intensity modulators, as shown in Figure 2.3(a), are constructed as an MZI in which one of the optical arms is phase modulated. In this specific configuration, a bias voltage, $V_{\text {bias }}$, is applied in addition to the modulating voltage, $V_{m}(t)$. If the voltages are applied to a single branch, the arrangement is called single-drive configuration. The spectrum of an intensity modulated optical carrier can be expressed as follows

$$
E_{I M}(t)=\frac{\beta}{2} e^{j\left(\omega_{0} t\right)}\left(e^{j\left(\frac{\pi V_{b i a s}}{V_{\pi, D C}}+\frac{\pi V_{R F}}{V_{\pi, R F}} \sin \left(\omega_{R F} t\right)\right)}-1\right),
$$




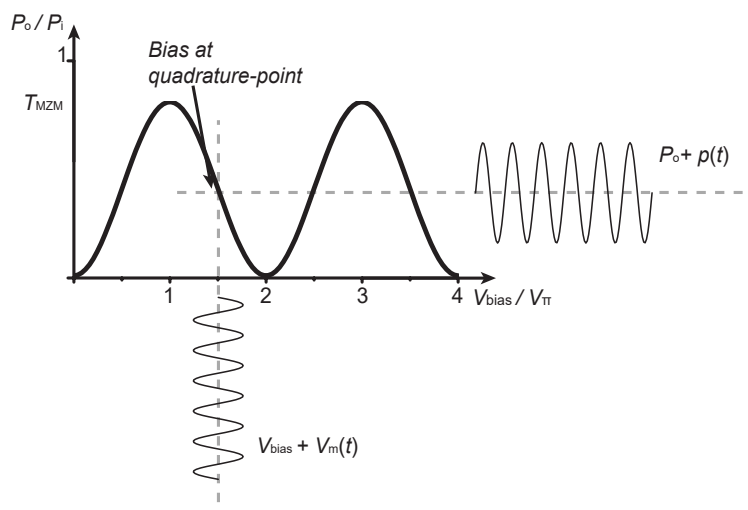

Figure 2.4: MZM transfer characteristic

where $\beta=\left(\gamma \sqrt{2 P_{i} / L_{M o d, l i n}}\right), L_{M o d, l i n}$ is the insertion loss of the modulator, $V_{\pi, D C}$ is the half-wave voltage specified in the stationary case, while $V_{\pi, R F}$ is the half-wave voltage specified at RF. The half-wave voltage is frequencydependent and the two notations used in (2.7), namely $V_{\pi, D C}$ and $V_{\pi, R F}$, are actually referring to the same parameter $V_{\pi}$ and the dependence on the frequency has been explicitly highlighted since especially $V_{\pi, R F}$ influences the overall RF performances of the MWP systems. According to the Jacobi-Anger expansion, the modulated optical field, when the modulator is biased at quadrature point, i.e., $V_{\text {bias }}=n V_{\pi, D C} / 2$ ( $n$ being an odd integer number), can be expressed as follows

$$
\begin{aligned}
E_{I M}(t) & =\frac{\beta}{2}\left[-\sum_{n=-\infty}^{\infty} J_{n}\left(\frac{\pi V_{R F}}{V_{\pi, R F}}\right) \sin \left(\omega_{0} t+n \omega_{R F} t\right)-\cos \left(\omega_{0} t\right)\right. \\
& \left.+j\left(\sum_{n=-\infty}^{\infty} J_{n}\left(\frac{\pi V_{R F}}{V_{\pi, R F}}\right) \cos \left(\omega_{0} t+n \omega_{R F} t\right)-\sin \left(\omega_{0} t\right)\right)\right]
\end{aligned}
$$

The spectrum of the intensity-modulated optical carrier is represented in Figure 2.3(b). The typical static power transmission characteristics of an MZM (i.e., with only a DC voltage applied) is given by:

$$
\frac{P_{o}}{P_{i}}=\frac{T_{M Z M, l i n}}{2}\left[1-\cos \left(\frac{\pi V_{\text {bias }}}{V_{\pi, D C}}\right)\right],
$$

and it is shown in Figure 2.4. In (2.9), $P_{o}$ and $P_{i}$ represent the output average optical power and the input average optical power, respectively. $T_{M Z M \text {,lin }}$ is the transmission coefficient of the modulator on a linear scale when it is biased for maximum transmission, which can also be expressed in terms of insertion loss, $L_{M Z M, l i n}=1 / T_{M Z M, l i n}$, or alternatively in $\mathrm{dB}$ as $T_{M Z M}=-L_{M Z M}$. It can be seen from (2.9) that, in addition to $L_{M Z M}$, an extra loss of $3 \mathrm{~dB}$ is present if the modulator is biased at the so-called quadrature-point. At this 
specific point, highlighted in Figure 2.4, the modulator works approximately in a quasi-linear regime, meaning that the modulating electric field and the optical output intensity are approximately linearly related to each other, if the modulating amplitude is small, i.e. $V_{R F}<<V_{\pi, R F}$. In order to keep the number of significant sidebands and therefore the bandwidth small, it is important to keep the modulation within the quasi-linear regime.

\subsubsection{Photodetection}

Any optical-to-electrical conversion, to bring optically encoded information back into the $\mathrm{RF}$ regime, relies on photodetection. A most convenient way of photodetection, which can also be integrated in photonic circuits, is using semiconductor photodiodes. A photodiode can only detect changes of the incoming optical power and it converts them into variations of a photocurrent. The most important parameters which describe the quality of a photodiode are the responsivity, $R_{P D}$ in $[\mathrm{A} / \mathrm{W}]$, the linearity, and the bandwidth. Another important property is the capability of handling high optical powers with a linear response. This is becoming increasingly important since at high optical power densities, non-linearities from the photodiode will negatively affect the APL performance.

Three different kinds of detection are available and might be used in MWP systems, namely direct detection, balanced detection and coherent detection. Direct detection is the simplest way to convert information back to RF, but is only possible in a system where intensity is not constant, for example when intensity modulation is used. Balanced detection is often used in systems where a larger amount of intensity noise is present because it is based on subtraction of correlated noise. Coherent detection is needed when information is encoded in the phase or frequency of the optical carrier. Balanced and coherent detection come at the cost of more complexity. Therefore, after the evaluation of the system, the proper way of detecting must be chosen. Here, direct detection and balanced detection will be analyzed, since those are the ones used in the systems presented in Chapter 6 and Chapter 7 .

\subsubsection{Direct detection}

A typical link based on intensity modulation (IM), where direct detection (DD) is used, known as IM-DD link, is shown in Figure 2.5(a). Here, a CW laser is modulated by the single-drive MZM, described in section 2.1.2.2. The output optical field $E_{I M}(t)$ illuminates the photodiode for direct detection. At the photodetector an impedance matching circuitry is used for connection with the load. For clarity of presentation, we limit the present section to low frequencies, to permit us to neglect the reactive components of the impedances, leaving only the resistive components. If the two resistances, $R_{M A T C H}$ and $R_{L A O D}$, are equal, the photocurrent is equally split in the two branches and the current 


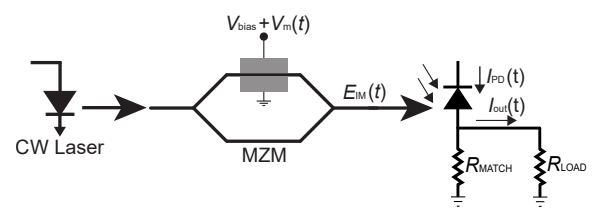

(a)

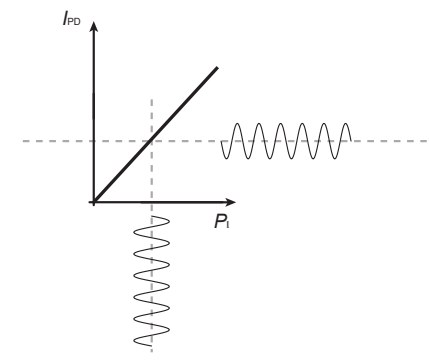

(b)

Figure 2.5: (a) Schematic for intensity modulated optical carrier and direct detection (IM-DD link), (b) Photodetector static transfer characteristic

on the load is half of the total photocurrent, which means that such matching circuitry is lossy since half of the current is drawn by the matched resistor. The ideal behavior in terms of transfer characteristic of a photodiode is plotted in Figure 2.5(b), where $I_{P D}$ is the photocurrent, $P_{I}$ is the input optical power and the slope is given by the responsivity, $R_{P D}=I_{P D} / P_{I}$. The incident optical power is proportional to the absolute square of the optical field amplitude, $E_{I M} \cdot E_{I M}^{\star}$, as expressed by

$$
I_{P D}=R_{P D} P_{I}=R_{P D} \frac{E_{I M}(t) \cdot E_{I M}^{*}(t)}{2 \gamma^{2}},
$$

where $\gamma$ is a constant relating the field and the average power. The current in the load, due to the impedance match, is given as follows

$$
I_{\text {out }}(t)=\frac{1}{2} I_{P D}(t)
$$

In (2.10), from the cancellation of the complex exponential factors, it can be seen that direct photodetection results in loss of phase information. Therefore direct detection cannot detect any phase modulation, but only intensity modulation.

\subsubsection{Balanced detection}

Balanced detection is achieved using a so-called balanced photodetector, which consists of a pair of photodiodes as shown in Figure 2.6(a). The output photocurrent of the balanced detector, beacuse it is picked up between the photodiodes, is simply the difference of the currents generated by each photodiode, which are $I_{P D 1} \propto E_{1}(t) \cdot E_{1}^{\star}(t)$ and $I_{P D 2} \propto E_{2}(t) \cdot E_{2}^{\star}(t)$. Assuming that the detection is perfectly balanced, i.e., that the two photodiodes have identical responsivity, $R_{P D}$, and are illuminated with equal power fractions, the output 


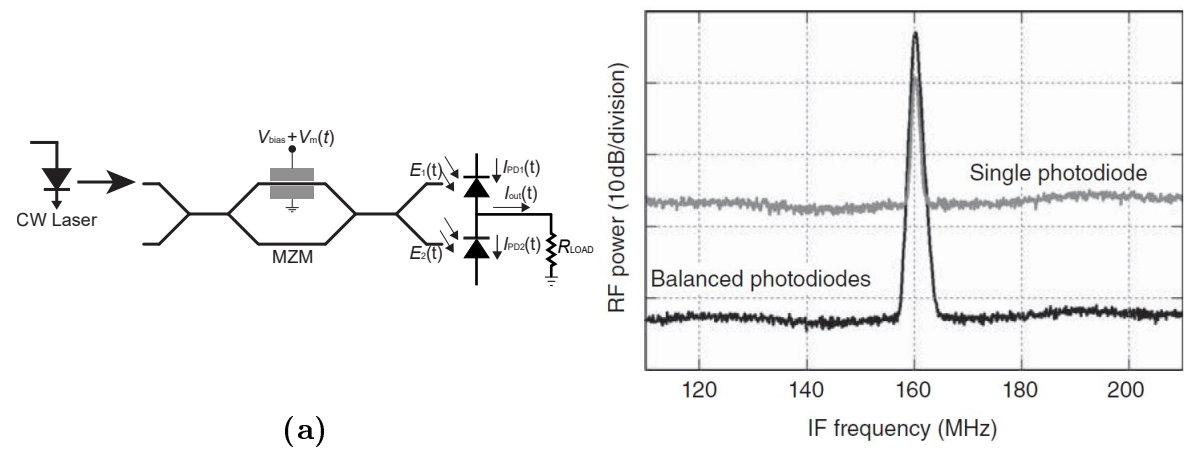

(b)

Figure 2.6: (a) Analog photonic link based on intensity modulation and balanced detection, (b) Measured outputs of a $12 \mathrm{GHz}$ down-converting link based on intensity modulation and using a single photodiode (gray) and balanced photodiodes (black) (picture edited from [15])

photocurrent, $I_{\text {out }}$, can be expressed as

$$
I_{\text {out }}=I_{P D 1}-I_{P D 2} \text {. }
$$

Substracting the two currents will lead to the rejection of what is called common mode noise, i.e., intensity noise that follows the same fluctuations in both detector branches. Therefore the noise floor in balanced detection can be much reduced compared to the case of direct detection with a single detector, as shown in Figure 2.6(b), where the RF output power using a single photodiode is compared to the RF output power when using balanced detection. Thus, using balanced detection can further improve the APL performance [16], [17]. Also here, as already explained in section 2.1.3.1, a lossy impedance matching circuitry is used in most of the cases, which means that the current to the load is half of the current given by the sutraction of the two photocurrents of the photodiodes.

\subsection{Analog photonic links and performance}

In this section the definitions of the performance parameters of an APL are given. The most important parameters, which will be addressed belowe are the link gain, the noise figure, the non-linearities and the spurious-free dynamic range (SFDR). These parameters will be extensively used in the next chapters, when the implemented MWP systems will be characterized. For those who are familiar with RF parameters used to characterize amplifier or other devices, these parameters are known. The use of the same concepts as the ones used in RF domain is licit since both the inputs and outputs of an APL are in the RF 


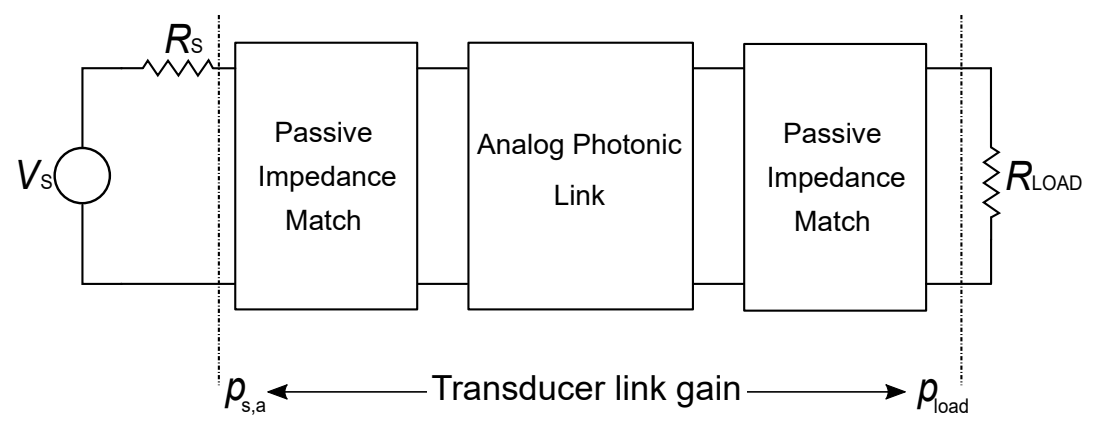

Figure 2.7: APL connected with a source and a load

domain.

\subsubsection{Link gain}

Any APL can be characterized using the link gain, $g$, a fundamental parameter that describes how efficiently the power of the RF signal is transferred through the system. A block diagram of an APL is shown in Figure 2.7, where also the circuitry for transfering the available power at the source to the APL, or more precisley to the modulator, and the circuitry for transfering the photocurrent to the load resistor are depicted. When a system is based on an APL, the most applicable definition of link gain is the transducer power gain, which is defined as the ratio of the power delivered to the load, $p_{l o a d}$, to the power available from the source, $p_{s, a}$, therefore

$$
g=\frac{p_{l o a d}}{p_{s, a}}
$$

In Figure 2.7, the reference planes, at which the powers mentioned before are referring at, are shown. From now on, since we will only use the transducer power gain definition for the systems presented in this thesis, we will omit the words transducer and power, and we will refer to this quantity as link gain, and often, especially in the measurements, the link gain will be given in $\mathrm{dB}$ as

$$
G=10 \log (g)
$$

\subsubsection{Noise in an analog photonic links}

One of the most important aspect that affects the quality of a communication system and hence an APL as well depends on how accurately the received signal reproduces the message sent by the source. In a real system though, the message (information) is always disturbed by noise, which limits the performance because noise is combined with the desired signal such that the behavior in time 


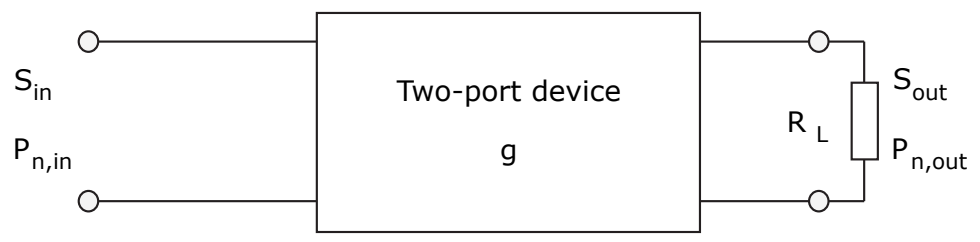

Figure 2.8: Circuit representation for noise figure evaluation

changes and therefore the received signal will differ from the original one. Let us consider Figure 2.8. Here, we have a circuit representation of a two-port device, characterized by a cartain gain, $g$. What has to be quantified is how much noise is added to the transmitted signal by the noisy two-port device. This is usually done via the ratio of the output signal power, $S_{\text {out }}$, to the output noise power, $P_{n, \text { out }}$, a quantity known as signal-to-noise ratio (SNR),

$$
S N R_{\text {out }}=\frac{S_{\text {out }}}{P_{n, \text { out }}} .
$$

The output noise power can be calculated as the product of the power spectral density (PSD) of the output noise and the bandwith over which the noise is measured, $B_{n}$, as $P_{n, \text { out }}=N_{\text {out }} B_{n}$. In order to quantify the degradation of the signal when it is transmitted by the two-port device, the input SNR must be compared to the output SNR. Let us consider the case graphically described by Figure 2.8, where the two-port device is first assumed to be noiseless. In this case the output SNR is as follows

$$
\frac{S_{\text {out }}}{P_{n, \text { out }}}=\frac{g S_{\text {in }}}{g P_{n, \text { in }}}=\frac{S_{\text {in }}}{P_{n, \text { in }}},
$$

where $S_{i n}$ is the input signal power and $P_{n, i n}$ is the input noise power, when the input termination is at standard noise temperature. In the noiseless ideal scenario the output SNR is the same as the input SNR, since there is no degradation. Instead, for the noisy case, the output SNR becomes

$$
\frac{S_{\text {out }}}{P_{n, \text { out }}}=\frac{g S_{\text {in }}}{g\left(P_{n, \text { in }}+P_{n, a}\right)}=\frac{S_{\text {in }}}{P_{n, \text { in }}+P_{n, a}},
$$

where $P_{n, a}$ is the additional noise power generated by the two-port device referred to the input. Practically, an easily measurable quantity which describes the reduction of the output SNR when compared to the input SNR, is the noise factor, $F$, defined as follows

$$
F=\frac{S_{\text {in }} / P_{n, \text { in }}}{S_{\text {out }} / P_{n, \text { out }}}=\frac{S N R_{\text {in }}}{S N R_{\text {out }}}=\frac{S_{\text {in }} /\left(N_{\text {in }} B_{n}\right)}{S_{\text {out }} /\left(N_{\text {out }} B_{n}\right)} .
$$




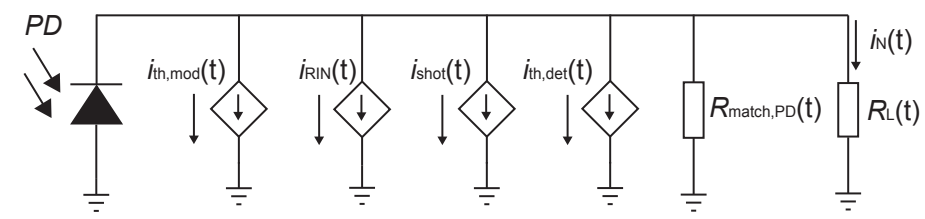

Figure 2.9: Circuit representation of noise source as current sources

The noise factor is commonly converted into decibels and it is referred to as the noise figure, $N F$, as follows

$$
N F=10 \log _{10} F .
$$

The APL can be inserted in Figure 2.8 as the two-port noisy device.

Noise can be categorised in a number of ways. However the three main types, which arise in an APL and will be part of the output noise power, $P_{n \text {,out }}$, of the APL are thermal noise, shot noise and RIN. First, we will mathematically describe the three types of noise, and later on we will introduce the most important parameters that are used to quantify the quality of the APL in terms of noise.

A circuit representation of noise sources as current sources is shown in Figure 2.9. Here, the noise terms are modeled as current sources and a lossy impedance matching has been used to maximize the power transfer to the load $\left(R_{\text {match }, P D}=R_{L}\right)$. The noise currents are assumed to be wide-sense stationary, ergodic and statistically independent of each other [18]. Therefore, the total noise power can be calculated as the sum of noise powers generated by the individual sources. Furthermore, because of the stationarity and ergodicity, the statistical properties can be calculated from the temporal average. Considering the lossy impedance matching as in Figure 2.9, the total noise current through the load can be written as

$$
i_{N}(t)=\frac{1}{2}\left(i_{t h, \bmod }(t)+i_{I N}(t)+i_{\text {shot }}(t)+i_{\text {th, det }}(t)\right) .
$$

From [19], the variances of the individual current terms are expressed as follows

$$
\begin{aligned}
\left\langle i_{\text {th }}^{2}\right\rangle & =\frac{4 k_{B} T B_{n}}{R}, \\
\left\langle i_{\text {shot }}^{2}\right\rangle & =2 q I_{a v} B_{n}, \\
\left\langle i_{\text {rin }}^{2}\right\rangle & =10^{\frac{R I N}{10}} I_{a v}^{2} B_{n},
\end{aligned}
$$

where in $(2.21) k_{B}=1.38 \cdot 10^{-23} \mathrm{~J} / \mathrm{K}$ is the Boltzmann constant, $T$ is the absolute temperature. In (2.22) $q$ is the electron charge, while RIN in (2.23) has been defined in (2.2) and it is assumed constant over the bandwidth of 
interest. $I_{a v}$ is the average photocurrent and $B_{n}$ the bandwidth in Hertz over which the noise is measured. The output noise PSD can be written as

$$
N_{\text {out }}=\left(N_{t h, \text { det }}+g N_{t h, \text { mod }}+N_{\text {shot }}+N_{R I N}\right) .
$$

where $N_{\text {shot }}, N_{R I N}$ and $N_{t h, d e t / m o d}$, expressed in $\mathrm{dB} / \mathrm{Hz}($ or $\mathrm{dBm} / \mathrm{Hz})$, represent respectively the PSD of the shot noise, the PSD of the relative intensity noise and the PSD of the thermal noise. It has to be noticed that there are two terms related to the thermal noise, namely, $N_{t h \text {,det }}$ is originating from the photodetector, while $N_{t h, \text { mod }}$ arises from the modulator and it is multiplied by the link gain $g$ because it is originally at the link input while the noise power is evaluated at the output of the link. The expression for the PSDs are evaluated considering the impedance matching, therefore using $N_{x}=\frac{1}{4} \frac{1}{B_{n}}\left\langle i_{x}^{2}\right\rangle R_{L}$, and are as follows

$$
\begin{aligned}
N_{t h, d e t} & =k_{B} T, \\
N_{t h, \text { mod }} & =k_{B} T, \\
N_{\text {shot }} & =\frac{1}{2} q I_{a v} R_{L}, \\
N_{R I N} & =\frac{1}{4} 10^{\frac{R I N}{10}} I_{a v}^{2} R_{L} .
\end{aligned}
$$

Inserting in (2.18) the input noise PSD, $N_{i n}$, which is due to the thermal noise from a matched resistive load, $N_{i n}=k_{B} T$, the output signal power, $S_{\text {out }}$, which is given by $S_{\text {out }}=g S_{\text {in }}$, and the total output noise PSD, $N_{\text {out }}$, given in (2.24), the noise factor can be calculated as

$$
F=\frac{S_{\text {in }} /\left(k_{B} T B_{n}\right)}{g S_{\text {in }} /\left(N_{\text {out }} B_{n}\right)}=\frac{N_{\text {out }}}{g k_{B} T} .
$$

The noise figure is then

$$
N F=N_{\text {out }}[d B m / H z]-G+174[d B m / H z] .
$$

\subsubsection{Non-linearities}

Earlier in this chapter some assumptions have been made considering that both the modulator and the photodetector are working in the linear or quasi-linear region of their trans-characteristics and therefore a small-signal analysis can be carried out. However, modulation devices are intrinsically non-linear and in order to have a complete view over the APL link performance, non-linearities have to be investigated. For the purpose of this work, we will treat static weak non-linearities [20], which means non-linearities that have no memory effect and that the amplitudes of the generated distorsion products depend only on the amplitude of the input signal and not on the frequency. With this 


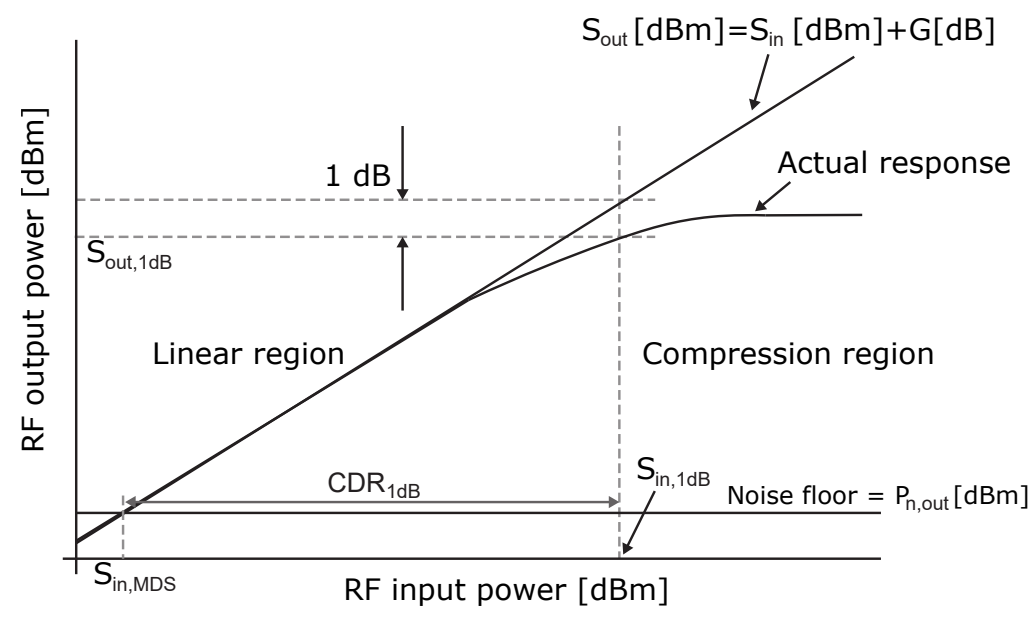

Figure 2.10: Representation of compression dynamic range on a log-log scale. The compression point at $1 \mathrm{~dB}$ is highlighted, where the actual response of the APL link deviates $1 \mathrm{~dB}$ from the linear response

assumptions, some figures of merit (as amplitudes or amplitude ratios) can be calculated using a Taylor series of the nonlinearity

$$
y(x)=\sum_{n=0}^{\infty} a_{n}\left(x-x_{0}\right)^{n}
$$

where $\mathrm{x}$ is the input signal and $a_{n}$ are the expansion coefficients given by

$$
a_{n}=\left.\frac{1}{n !}\left(\frac{d^{n} y(x)}{d x^{n}}\right)\right|_{x=x_{0}}
$$

There are two main tests for evaluating the performance of an APL link in terms of non-linear behavior: single-tone and two-tone test; the analysis can go up to multi-tone analysis, but for the purpose of this work, we will keep our analysis to the two-tone test.

\subsubsection{Single-tone test: harmonic distortions and compression dy- namic range}

Through the single-tone test, some important conclusions can be drawn about harmonic distorsion (HD) and gain compression. A single-tone input is written as

$$
x(t)=x_{0}+A \cos (\omega t)
$$

where $\omega=2 \pi f$ is the angular fundamental frequency, $A$ is the amplitude of the signal and $x_{0}$ is the bias current or voltage. Having harmonic distortion means 
the presence of multiples of a fundamental frequency of interest. Substituting (2.33) in (2.31) and evaluating up to $n=3$ yields

$$
\begin{aligned}
y(t) & =a_{0}+\frac{1}{2} a_{2} A^{2}+\left(a_{1} A+\frac{3}{4} a_{3} A^{3}\right) \cos (\omega t) \\
& +\frac{1}{2} a_{2} A^{2} \cos (2 \omega t)+\frac{1}{4} a_{3} A^{3} \cos (3 \omega t) .
\end{aligned}
$$

The spurious components with frequencies of integer multiple of the fundamental frequency are addressed as harmonic distortions, where the $n$-th harmonic describes the $n$-order harmonic distortion. The disadvantages of having HDs are basically two, namely waste of power in harmonics and interference from harmonics. The HDs closest in frequency to the fundamental are the HDs of the second-order. Usually they are neglected because they still fall relatively far from the fundamental frequency, and thus often out of the operational bandwidth. Nevertheless, this is only true for narrow-band systems with suboctave operational bandwidth (where the highest frequency is less than twice the lowest frequency). But when wide-band systems are used, $\mathrm{HD}_{2}$ becomes significant and therefore has to be taken into account. This last consideration is extremely important for APLs since the operational band can span in principle from few $\mathrm{MHz}$ to hundreds of GHz. However, in our systems, presented in the next chapters, we still use bandwidths where the HDs of the second order can be neglected.

Another important parameter that can be calculated by the single-tone test is the compression dynamic range (CDR). A graphical representation is given in Figure 2.10, where the output power is plotted against the input power. The $1-\mathrm{dB} C D R\left(\mathrm{CDR}_{1 \mathrm{~dB}}\right)$ is the range of input powers which produces an output signal above the noise floor until the output power is compressed by $1 \mathrm{~dB}$ with respect to a linear response. The so-called noise floor can be retrieved from (2.30) as

$$
\begin{aligned}
P_{n, \text { out }}[d B m] & =N_{\text {out }}[d B m]+10 \log _{10} B_{n} \\
& =N F[d B]+G[d B]-174[d B m / H z]+10 \log _{10} B_{n} .
\end{aligned}
$$

A signal weaker than this will be lost in the noise. The minimum detectable signal is generally taken as $3 \mathrm{~dB}$ higher than the value given by (2.35) and the corresponding input power, $S_{i n, M D S}$, is calculated to be

$$
\begin{aligned}
S_{i n, M D S}[d B m] & =P_{n, \text { out }}[d B m]+3-G[d B] \\
& =N F[d B]-171[d B m / H z]+10 \log _{10} B_{n} .
\end{aligned}
$$

From inspection of Figure 2.10, the input power at the 1-dB compression point is given by

$$
S_{\text {in }, 1 d B}[d B m]=S_{\text {out }, 1 d B}[d B m]-G[d B]+1 .
$$

The difference between the input power at the 1-dB compression point and the 
minimum detectable signal defines the 1-dB compression dynamic range as

$$
\begin{aligned}
C D R_{1 d B}[d B m] & =S_{\text {in, } 1 d B}[d B m]-S_{\text {in }, M D S}[d B m] \\
& =S_{\text {out }, 1 d B}[d B m]-G[d B]-N F[d B]+172[d B m / H z] \\
& -10 \log _{10} B_{n}
\end{aligned}
$$

\subsubsection{Two-tone test: intermodulation distortions and spurious- free dynamic range}

A two-tone test can be used to characterize the intermodulation distorsion (IMD) and the SFDR. A signal composed of two sine waves with equal amplitudes and fundamental angular frequencies $\omega_{1}$ and $\omega_{2}$ is expressed as

$$
x(t)=x_{0}+A \cos \left(\omega_{1} t\right)+A \cos \left(\omega_{2} t\right) .
$$

Once again the same process as for the single-tone test is done, substituting (2.39) in (2.31) and evaluating up to $n=3$ yields

$$
\begin{aligned}
y(t) & =a_{0}+a_{2} A^{2}+\left(a_{1} A+\frac{9}{4} a_{3} A^{3}\right)\left(\cos \left(\omega_{1} t\right)+\cos \left(\omega_{2} t\right)\right) \\
& +\frac{1}{2} a_{2} A^{2}\left(\cos \left(2 \omega_{1} t\right)+\cos \left(2 \omega_{2} t\right)\right)+\frac{1}{4} a_{3} A^{3}\left(\cos \left(3 \omega_{1} t\right)+\cos \left(3 \omega_{2} t\right)\right) \\
& +a_{2} A^{2}\left(\cos \left(\left(\omega_{1}-\omega_{2}\right) t\right)+\cos \left(\left(\omega_{1}+\omega_{2}\right) t\right)\right) \\
& +\frac{3}{4} a_{3} A^{3}\left[\cos \left(\left(2 \omega_{1}-\omega_{2}\right) t\right)+\cos \left(\left(2 \omega_{2}-\omega_{1}\right) t\right)\right. \\
& \left.+\cos \left(\left(2 \omega_{1}+\omega_{2}\right) t\right)+\cos \left(\left(2 \omega_{2}+\omega_{1}\right) t\right)\right] .
\end{aligned}
$$

The result of a two-tone test is not just the harmonics of each sine wave, but also components at the sums and differences of the harmonics and the fundamental frequencies. A typical power spectrum for a two-tone test is shown in Figure 2.11, where one can recognize HDs and IMDs, which are the terms given by the sums and differences of the harmonics and the fundamental frequencies. Two of the most challenging distortion products are the signal content due to third-order distortion that occurs directly adjacent to the two input tones at $\left(2 f_{1}-f_{2}\right)$ and $\left(2 f_{2}-f_{1}\right)$, where they cannot be filtered out.

We note that in an APL, especially when used as processing core in an RF telecommunications system, as in our case, IMDs of the third order are extremely important for a range of reasons. In modulated signals, third-order distortion creates additional frequency content in bands adjacent to the modulated signal. In a transmitter, the additional frequency components resulting from poor linearity can interfere with other adjacent channels. In a receiver, intermodulation distorsions can cause out-of-band signals that might obscure the signal of interest. In most of the cases the performance of an APL in terms of linearity is given by the $\mathrm{SFDR}_{n}$, which is defined as the range of input 


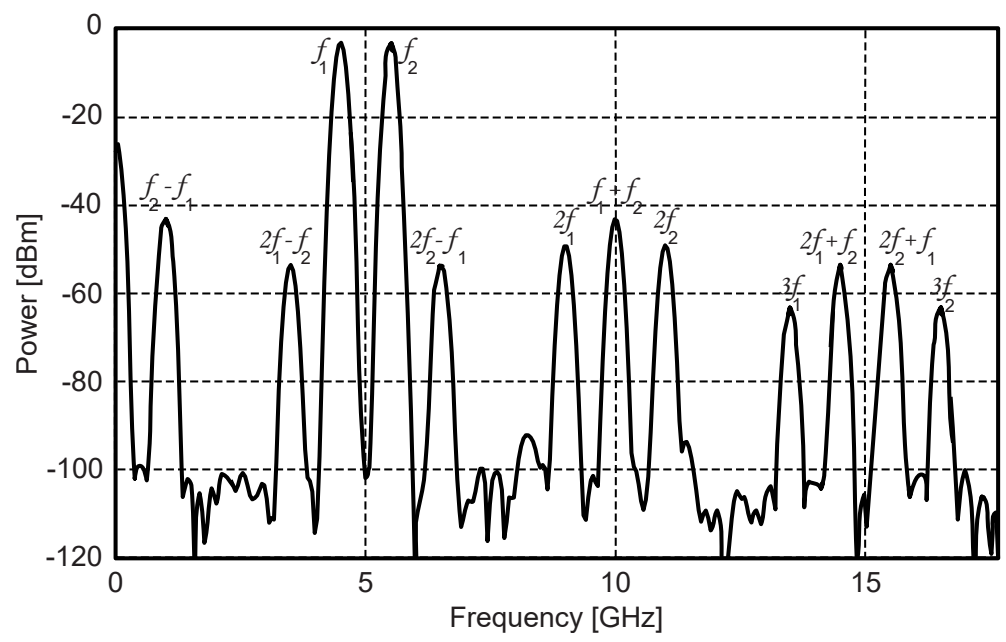

Figure 2.11: Output power spectrum resulting from a two-tone test simulation

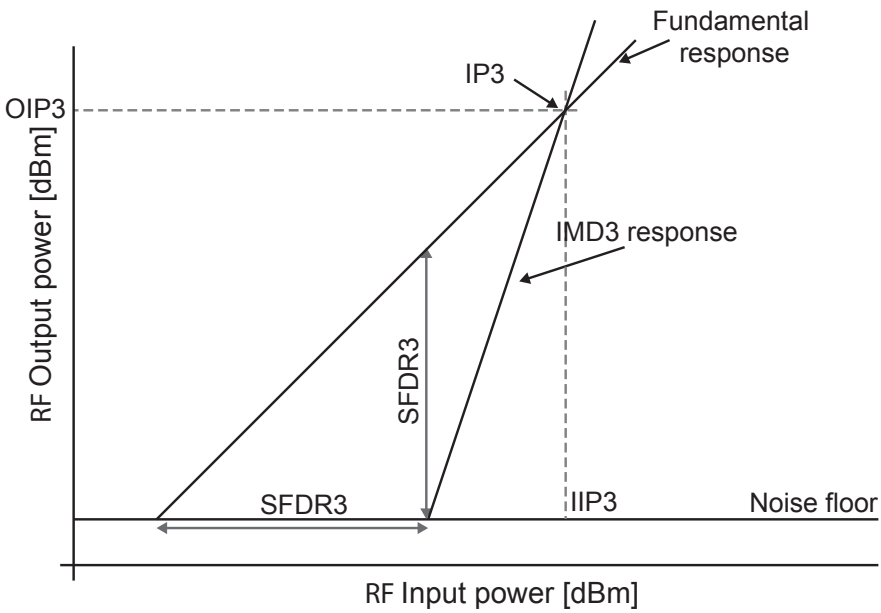

Figure 2.12: Representation of spurious-free dynamic range in a log-log scale. As example the linear fundamental response with slope equal to $1 \mathrm{~dB} / \mathrm{dB}$ and the $\mathrm{IMD}_{3}$ response with slope equal to $3 \mathrm{~dB} / \mathrm{dB}$ are plotted. The output intercept point of the third order $\left(\mathrm{IP}_{3}\right)$ is shown. The $\mathrm{x}$ and $\mathrm{y}$ coordinate of the $\mathrm{IP}_{3}$ are respectively the input intercept point, $\mathrm{IIP}_{3}$, and the output intercept point, $\mathrm{OIP}_{3}$. 
powers over which the output signal is above the output noise floor and all the spurious signals are less than the output noise floor. A graphical representation is given in Figure 2.12, where the response of the fundamental signal and the intermodulation products of the third order are plotted in a dB-scale graph. Here, the two curves will meet at a point where the intermodulation distorsion of the third-order equals in amplitude the first-order signal. This is the thirdorder intercept point (IP3). It is a theoretical point that is never achieved in practice, because of the saturation of the non linear devices. However, it is useful in quantifying the limits to linearity. The IP3 value can be retrieved with reference to the input or the output. If one reads the value from the output axis, it is called output intercept point $(\mathrm{OIP})_{3}$. The value read from the input axis, instead, is called input intercept point (IIP) $)_{3}$. It is interesting to define the $\mathrm{SFDR}_{3}$ in terms of $\mathrm{OIP}_{3}$ or $\mathrm{IIP}_{3}$, such that practically, with only one measurement, the SFDR can be determined. The expression for the $\mathrm{SFDR}_{3}$ in terms of $\mathrm{OIP}_{3}$ and $\mathrm{IIP}_{3}$ is as follows

$$
\begin{aligned}
S F D R_{3}[d B] & =\frac{2}{3}\left[O I P_{3}[d B m]-N_{\text {out }}[d B m / H z]-10 \log _{10} B_{n}\right] \\
& =\frac{2}{3}\left[I I P_{3}[d B m]+G[d B]-N_{\text {out }}[d B m / H z]-10 \log _{10} B_{n}\right] .
\end{aligned}
$$

where $N_{\text {out }}$ is the noise PSD calculated in (2.24), $B_{n}$ is the measurement bandwidth and $\mathrm{G}$ is the link gain. Substituting $N_{\text {out }}$ from (2.30) in (2.41), the $S F D R_{3}$ can be expressed as a function of the noise figure, as

$$
\begin{aligned}
S F D R_{3}[d B] & =\frac{2}{3}\left[O I P_{3}[d B m]-N F-G+174[d B m / H z]-10 \log _{10} B_{n}\right] \\
& =\frac{2}{3}\left[I I P_{3}[d B m]-N F+174[d B m / H z]-10 \log _{10} B_{n}\right] .
\end{aligned}
$$

To sum up, IIP3 tells how large a signal can be before IMD occurs. The higher the output at the intercept point, the better the linearity and the lower the IMD. 


\section{CHAPTER 3}

MODELLING OF MICROWAVE PHOTONIC FILTERS

\subsection{Introduction}

In this section we recall how to use a well-known tool, developed for the description of time-periodic electronic signals [21], the $z$-transform, for describing optical signal transfer through passive optical components, such as optical filters. The usage is licit since all the passive optical filters are linear and mostly time-invariant systems. Therefore, linear system theory can be applied for analyzing optical filters in time and frequency domains. It has to be noticed that in the case of optical filters, the input signals are continuous in time, but still the optical filters can be modelled as discrete time filters, as it will be described in the next sections.

The $z$-transform concept can be applied to any photonic signal processing

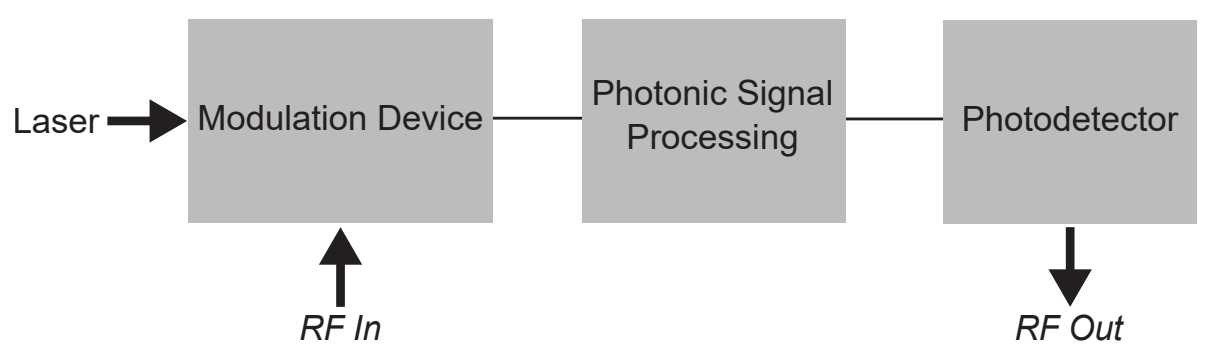

Figure 3.1: Schematic of an APL comprising the photonic signal processing core 
core of an APL, as shown in Figure 3.1. Here, we address several optical components and filters with the aid of the $z$-transform, presented in growing order of complexity. Per each filter, the transfer response (for single-input single-output) and the transfer matrix (for multiple inputs and outputs) in terms of $z$-transform will be given.

\section{$3.2 \quad Z$-Transform}

Optical filters can be described with the aid of the $z$-transform, since they can be convienently modelled as discrete system. In fact, each optical filter is characterized by a basic delay time, $T_{\text {delay }}$, such that any other delay present in the system containing the filter, is an integer multiple of this basic delay time. Specifically in their description, the impulse response of a general system is a series of output impulses which are equally spaced in time. This is valid for coherent interference or in other words when the longest delay time is much shorter than the coherence time of the source. Each optical filter can be characterized by the free spectral range (FSR), which is one period of the optical frequency reponse and is defined as

$$
F S R=\frac{1}{T_{\text {delay }}}=\frac{c}{n_{g} L_{\text {delay }}},
$$

where $c$ is the speed of light in vacuum, $L_{\text {delay }}$ is the corresponding length to the unit delay, $T_{\text {delay }}$, and $n_{g}$ is the group index , defined as

$$
n_{g}=n_{e f f}\left(f_{0}\right)+\left.f_{0} \frac{d n_{e f f}}{d f}\right|_{f_{0}},
$$

where $n_{\text {eff }}$ is the effective index of the material of which the optical components are made.

The $z$-transform for a discrete-time system, which is described by the impulsive response $h[n]$, is defined as

$$
H(z)=\sum_{n=-\infty}^{\infty} h[n] z^{-n},
$$

where $z$ is a complex variable. The $z$-transform is an extension of the well known discrete-time Fourier transform (DTFT), $H(f)$, which is related to the former as

$$
H(f)=\sum_{n=-\infty}^{\infty} h[n] e^{-j 2 \pi n f T_{\text {delay }}}
$$

It is easy to see that

$$
H(f)=\left.H(z)\right|_{z=e^{j 2 \pi f T_{\text {delay }}}}
$$




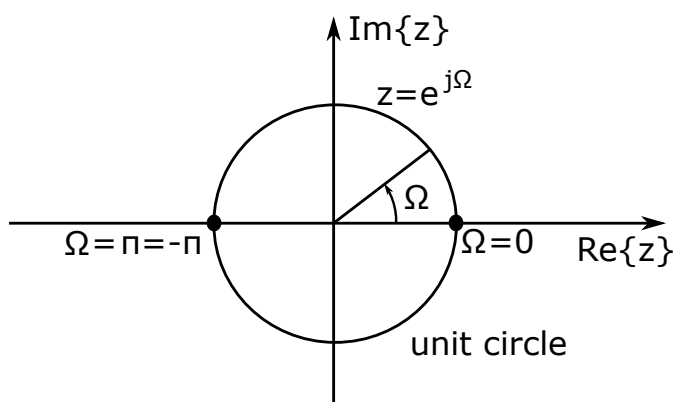

Figure 3.2: Representation of the unit circle in the $z$ complex plane. The vector $e^{j \Omega}$, pointing at an angle with regard to the horizontal axis, rotates with an angular velocity equal to $\Omega$.

The discrete-time Fourier transform (DTFT) corresponds to the $z$-transform when it is evaluated along the unit circle, which is plotted in Figure 3.2 in the complex plane. The unit circle is the locus described by the rotating vector, which has a modulus equal to one and a rotation angle equal to the normalized angular frequency, $\Omega$, defined as

$$
\Omega=2 \pi f T_{\text {delay }}
$$

The normalized frequency, or angular frequency will be extensively used in the next sections. Furthermore, it is common to use the power transfer response, which is the square of the absolute of $(3.4),|H(\Omega)|^{2}$, in order to describe the building blocks presented next. It must be kept in mind that (3.5) is valid only if the unit circle is included in the region of convergence (ROC) of the $z$-transform, which is defined as the region in the complex $z$-plane where the $z$-transform converges [21].

Another way of representing a linear and time-invariant system is by using a differential equation as

$$
y[n]+\sum_{k=1}^{N-1} a_{k} y[n-1]=\sum_{m=0}^{M-1} b_{m} x[n-m],
$$

where $y[n]$ is the output and $x[n]$ is the input of the system, while $a_{k}$ and $b_{m}$ are coefficients which depend on the system physical properties. Using the shifting property of the $z$-transform (i.e., if $\mathcal{Z}\{y[n]\}=Y(z)$, then $\mathcal{Z}\{a y[n-1]\}=$ $\left.a z^{-1} Y(z)\right)$, the $z$-transform of (3.7) becomes

$$
H(z)=\frac{Y(z)}{X(z)}=\frac{\sum_{m=0}^{M-1} b_{m} z^{-m}}{1+\sum_{k=1}^{N-1} a_{k} z^{-k}} .
$$

(3.8) can be expressed as the ratio of products of individual zero factors for the 
numerator and the denominator and therefore can be rewritten as

$$
H(z)=b_{0} z^{N-M} \frac{\prod_{m=1}^{M-1}\left(z-z_{m}\right)}{\prod_{k=1}^{N-1}\left(z-p_{k}\right)}
$$

where $z_{m}$ and $p_{k}$ are the zeros and poles of the $z$-transform, respectively. The zeros on the unit circle indicate that the amplitude response at that normalized frequency has zero transmission, while the location of poles determines whether the system is stable or not. The poles of a transfer function must all have a magnitude less than 1 in order for the system to have a stable, bounded response to a bounded input signal. Therefore, for a stable system all the poles lie inside the unit circle.

\subsubsection{Normalized group delay}

The frequency response is in general expressed as a complex function. This has the advantage that, along with the power transfer response, also the phase response, $\Phi(\Omega)=\arg (H(z))$, is taken into account. From the phase response, one can define the normalized group delay, $\tau_{g}^{\prime}$, which is the negative derivative of the phase response with respect to the normalized frequency, $\Omega$. The absolute group delay, $\tau^{\prime}$, is then found by multiplying the normalized group delay by the unit delay, $T_{\text {delay }}$. The absolute group delay is given in seconds, while the normalized group delay is given in number of roundtrips, as

$$
\tau_{g}^{\prime}=-\frac{d}{d \Omega} \Phi(\Omega)=-\left.\frac{d}{d \Omega} \arg (H(z))\right|_{z=e^{j \Omega}}
$$

\subsection{Modelling of photonic building blocks and filters}

All the optical components that are treated in this section comprise waveguide delays and directional couplers for splitting and combining the optical signals. Therefore, starting with the directional couplers, the optical filters that will be used later on in more complex systems will be analyzed using the $z$-transform in order to verify their frequency behavior.

\subsubsection{Directional coupler}

A schematic of a waveguide directional coupler is shown in Figure 3.3, where $\kappa$ is the power coupling coefficient associated with the directional coupler, and $c=\sqrt{1-\kappa}$ and $-j s=-j \sqrt{\kappa}$ are the field coefficients for the through- (from $E_{i, 1}$ to $E_{\text {out }, 1}$ and from $E_{i, 2}$ to $E_{\text {out }, 2}$ ), and cross- (from $E_{i, 1}$ to $E_{\text {out }, 2}$ and from $E_{i, 2}$ to $\left.E_{\text {out }, 1}\right)$ ports, respectively. The transfer matrix for a lossless directional 


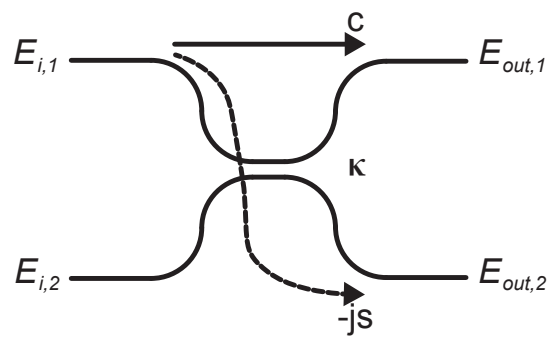

Figure 3.3: Schematic representation of a directional coupler with power coupling coefficient $\kappa$

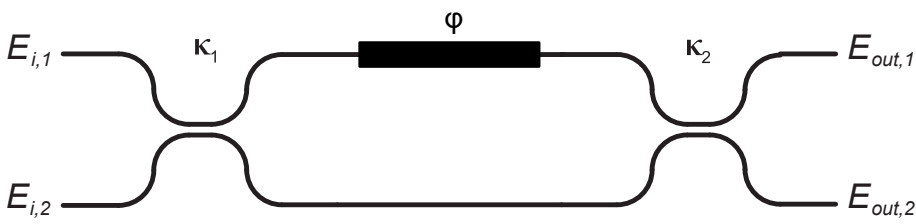

Figure 3.4: Schematic representation of a directional coupler with power coupling coefficient $\kappa$

coupler relates the outputs optical fields, $E_{\text {out }, 1}$ and $E_{\text {out }, 2}$ to the inputs optical fields, $E_{i n, 1}$ and $E_{i n, 2}$ and it is expressed as

$$
\left[\begin{array}{l}
E_{\text {out }, 1}(z) \\
E_{\text {out }, 2}(z)
\end{array}\right]=\left[\begin{array}{cc}
c & -j s \\
-j s & c
\end{array}\right]\left[\begin{array}{l}
E_{i, 1}(z) \\
E_{i, 2}(z)
\end{array}\right]
$$

It has been assumed that there is no wavelength dependency for the power coupling coefficient, therefore the elements of the transfer matrix are constant.

\subsubsection{Tunable coupler}

In many practical cases, it is necessary to make use of a tunable coupler (as a combiner or as a splitter), meaning that it should be possible to adjust the power coupling coefficient ideally from 0 to 1 . A tunable coupler can be obtained by using a symmetric MZI structure, as depicted in Figure 3.4, which comprises two cascaded directional coupler, described in section 3.3.0.1 connected by two optical waveguides having the same physical length, with one arm equipped with a tunable phase shifter, $\varphi$. The response of such a device does not depend on $z$, since there is no difference in length between the arms of the MZI. However, we can still define a transfer matrix for such a building 


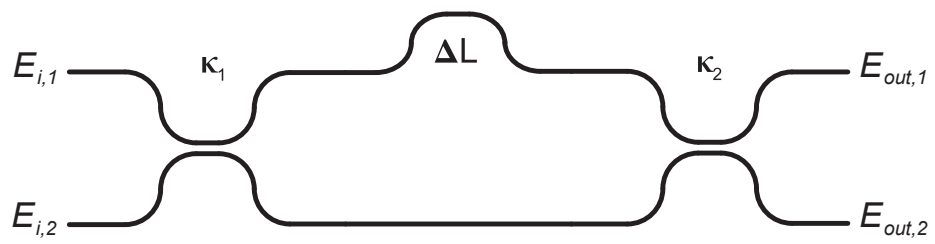

Figure 3.5: Schematic representation of an asymmetric Mach-Zehnder with $\Delta L$ length difference between the upper and lower branches

block, as

$$
\begin{aligned}
{\left[\begin{array}{l}
E_{\text {out }, 1}(z) \\
E_{\text {out }, 2}(z)
\end{array}\right] } & =\gamma\left[\begin{array}{cc}
c_{2} & -j s_{2} \\
-j s_{2} & c_{2}
\end{array}\right]\left[\begin{array}{cc}
e^{-j \phi} & 0 \\
0 & 1
\end{array}\right]\left[\begin{array}{cc}
c_{1} & -j s_{1} \\
-j s_{1} & c_{1}
\end{array}\right]\left[\begin{array}{l}
E_{i, 1}(z) \\
E_{i, 2}(z)
\end{array}\right] \\
& =\gamma\left[\begin{array}{cc}
\left(-s_{1} s_{2}+c_{1} c_{2} e^{-j \varphi}\right) & -j\left(s_{1} c_{2}+c_{1} s_{2} e^{-j \varphi}\right) \\
-j\left(s_{2} c_{1}+s_{1} c_{2} e^{-j \varphi}\right) & \left(c_{1} c_{2}-s_{1} s_{2} e^{-j \varphi}\right)
\end{array}\right]
\end{aligned}
$$

where $\gamma$ is the coefficient taking into account the waveguide loss and the phase contribution of the common path. This building block is extensively used in the optical beamforming networks that will be presented in the next chapters.

\subsubsection{Asymmetric Mach-Zehnder interferometer}

One of the simplest optical filters based on waveguides using a combination of directional or tunable couplers is the asymmetric Mach-Zehnder interferometer (AMZI), shown in Figure 3.5. It consists of two tunable couplers with power coupling coefficients equal to $\kappa_{1}$ and $\kappa_{2}$ and two waveguides connecting the two couplers ${ }^{1}$. The length different between the two waveguides branches is $\Delta L$ and is set to be the unit delay in time, $T_{\text {delay }}$, of the AMZI component. A $2 \times 2$ transfer matrix can be written as the product of the matrices of the first directional coupler, the basic unit delay and the second directional coupler as expressed next

$$
\begin{aligned}
{\left[\begin{array}{l}
E_{\text {out }, 1}(z) \\
E_{\text {out }, 2}(z)
\end{array}\right] } & =\gamma\left[\begin{array}{cc}
c_{2} & -j s_{2} \\
-j s_{2} & c_{2}
\end{array}\right]\left[\begin{array}{cc}
\alpha z^{-1} & 0 \\
0 & 1
\end{array}\right]\left[\begin{array}{cc}
c_{1} & -j s_{1} \\
-j s_{1} & c_{1}
\end{array}\right]\left[\begin{array}{l}
E_{i, 1}(z) \\
E_{i, 2}(z)
\end{array}\right] \\
& =\gamma\left[\begin{array}{cc}
\left(-s_{1} s_{2}+c_{1} c_{2} \alpha z^{-1}\right) & -j\left(s_{1} c_{2}+c_{1} s_{2} \alpha z^{-1}\right) \\
-j\left(s_{2} c_{1}+s_{1} c_{2} \alpha z^{-1}\right) & \left(c_{1} c_{2}-s_{1} s_{2} \alpha z^{-1}\right)
\end{array}\right],
\end{aligned}
$$

where $\gamma$ is the coefficient taking into account the waveguide loss and the phase contribution of the common path and $\alpha$ is the transmission coefficient considering the loss along the differential path. The transfer matrix contains four

\footnotetext{
${ }^{1}$ Note that in this case the couplers can be either directional or tunable. In most of the fabricated systems, we use tunable instead of directional couplers because of the possibility to reconfigure the filter.
} 
$z$-transform functions:

$$
\left[\begin{array}{l}
E_{\text {out }, 1}(z) \\
E_{\text {out }, 2}(z)
\end{array}\right]=\left[\begin{array}{ll}
H_{11}(z) & H_{12}(z) \\
H_{21}(z) & H_{22}(z)
\end{array}\right]\left[\begin{array}{l}
E_{i, 1}(z) \\
E_{i, 2}(z)
\end{array}\right],
$$

where $H_{11}(z)$ is the through-port response and $H_{22}(z)$ is its reverse polynomial, and $H_{12}(z)$ is the cross-port response and $H_{21}(z)$ is its reverse polynomial. The coefficients of the $z$-transform functions depend on the power coupling coefficients of the two directional couplers. Let us analyze the $z$-transform evaluated on the unit circle for the $H_{11}(z)$ and $H_{12}(z)$, neglecting the common path contribution $(\gamma=$ constant $)$ with $\kappa_{1}=\kappa_{2}=\kappa$. The two frequency responses are as follows

$$
\begin{aligned}
& H_{11}(\Omega)=-s^{2}+\alpha c^{2} e^{-j \Omega}, \\
& H_{12}(\Omega)=-j c s\left(1+\alpha e^{-j \Omega}\right) .
\end{aligned}
$$

Both the responses have a pole, referred to as trivial pole $e^{2}$ at the center of the complex plane. The corresponding phase responses are expressed as

$$
\begin{aligned}
& \Phi\left(H_{11}(\Omega)\right)=\arctan \left[\frac{-\alpha c^{2} \sin (\Omega)}{-s^{2}+\alpha c^{2} \cos (\Omega)}\right], \\
& \Phi\left(H_{12}(\Omega)\right)=\arctan \left[-\frac{c s+\alpha c s \cos (\Omega)}{\alpha c s \sin (\Omega)}\right] .
\end{aligned}
$$

The power transmission responses, i.e., the square of the absolute value of (3.15) and (3.16), are shown in Figure 3.6(a), respectively, while (3.17) and (3.18) are shown in Figure 3.6(b) together with the zero-pole diagram for both responses in Figure 3.6(c). It can be noticed that when the power coupling coefficients are equal to $0.5\left(\kappa_{1}=\kappa_{2}=0.5\right)$, both the $z$-transforms have a zero on the unit circle, meaning that they have a zero transmission at the corresponding normalized frequency. This is not always the case. The location of the zeros can vary depending on the values of the power coupling coefficients. As example, the power transmission and the corresponding phase response for three different combinations of $\kappa_{1}$ and $\kappa_{2}$ are depicted in Figure 3.7(a) and Figure 3.7(b), respectively, together with the zero-pole diagram in Figure 3.7(c). For both ports we can change the frequency at which the response is null adding a phase term, $e^{-j \varphi}$, before $z^{-1}$ and the result of a phase term change is depicted in Figure 3.6. By adjusting the phase term, is possible to place the zeros at any point on the complex plane. In order to quantify an AMZI in terms of rejection, it is common to define the ratio of maximum to minimum transmission over one FSR, known as Extinction Ratio (ER). It is important to notice that the

\footnotetext{
${ }^{2}$ Trivial because of two main reasons: one reason is that they correspond only to a time shift. The other is that if a system has a pole outside the unit circle, then certain bounded inputs will produce an unbounded output (unstable). But a pole at zero does not cause this unstable behavior, which is why it is called trivial.
} 


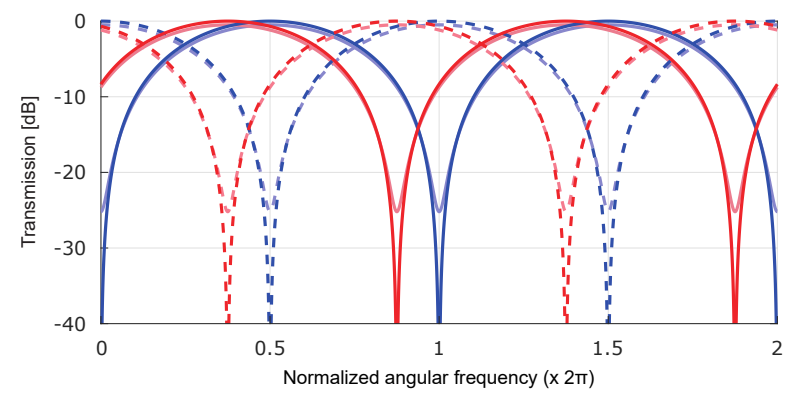

(a)

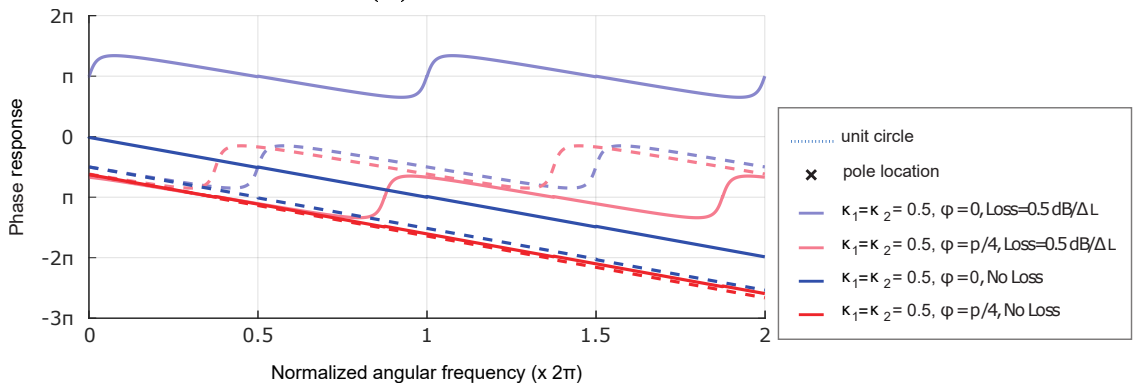

(b)
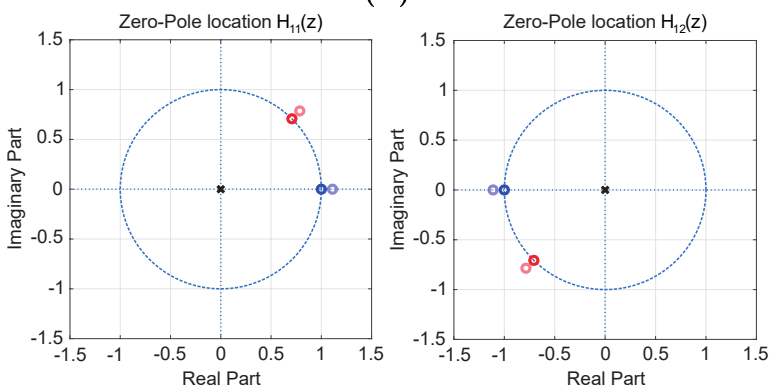

(c)

Figure 3.6: (a) Power transmission response, (b) phase response and zero-pole diagrams for an AMZI when $\kappa_{1}=\kappa_{2}=0.5$, considering the ideal lossless case and the lossy case. In the transmission response the solid line refers to the through-port, $H_{11}(f)$, while the dotted line is used for the cross-port, $H_{12}(f)$ 


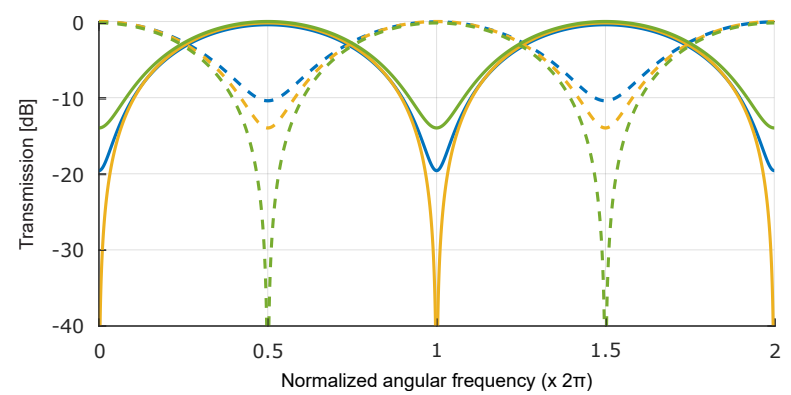

(a)

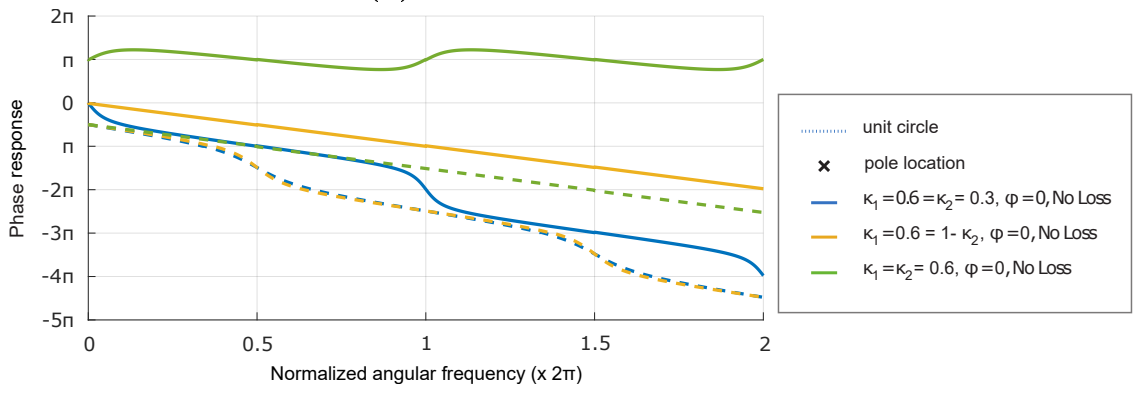

(b)
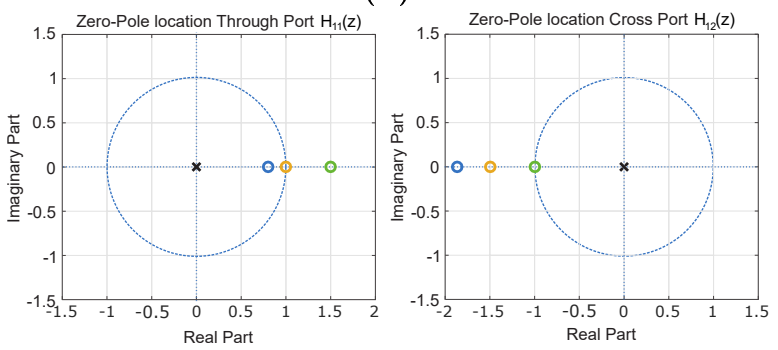

(c)

Figure 3.7: (a) Power transmission response, (b) phase response and zero-pole diagrams for an AMZI with different values of $\kappa_{1}$ and $\kappa_{2}$, considering the ideal lossless case. In the transmission response the solid line refers to the through-port, $H_{11}(f)$, while the dotted line is used for the crossport, $H_{12}(f)$ 


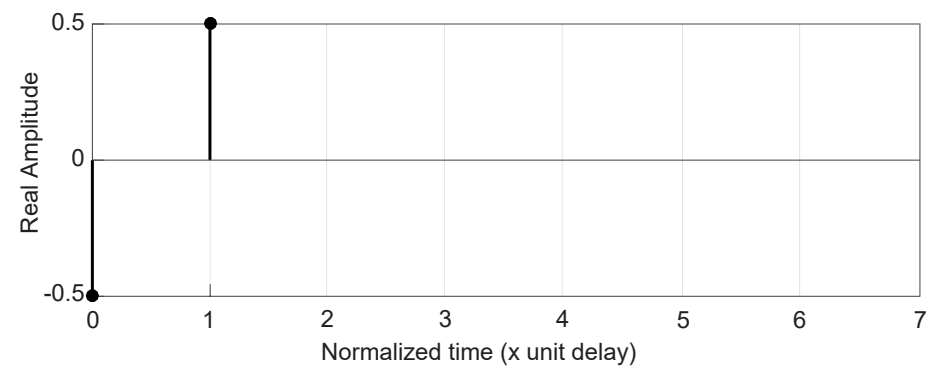

Figure 3.8: Impulsive Response of the through-port, $H_{11}(n)$ of an AMZI when $\kappa_{1}=$ $\kappa_{2}=0.5$

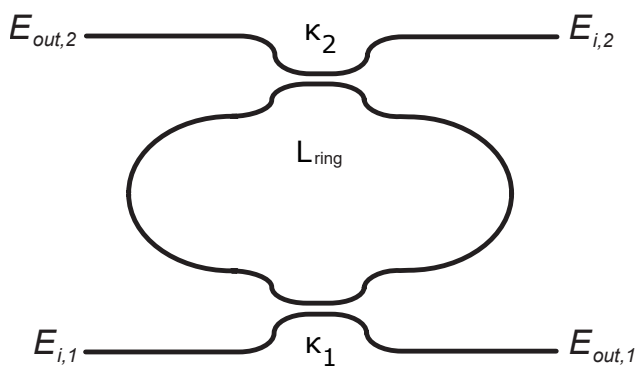

Figure 3.9: Schematic representation of a ring resonator

loss of the common path will lower the overall frequency response, while the loss of the differential path will also change the locations of poles and zeros, in both cases the ER will be lower than ideal case.

In terms of temporal behavior an AMZI provides a finite impulse response (FIR), which means that the response at the impulse has a limited number of taps, as shown in Figure 3.8. Here, the impulse response of the through-port, $H_{11}(n)$, is shown. The number of taps is two, one at 0 , which corresponds to the signal through the short branch, and the second tap at normalized time 1, which corresponds to the signal delayed by one differential length.

\subsubsection{Optical ring resonator}

A ring resonator (RR), shown in Figure 3.9 is an optical filter, which has two optical inputs, $E_{i, 1}$ and $E_{i, 2}$, and two optical outputs, $E_{\text {out }, 1}$ and $E_{\text {out }, 2}$. 
Assuming that only the input optical field $E_{i, 1}$ is applied, therefore $E_{i, 2}$ is zero, the $z$-transform for the so-called through-port response, $H_{11}(z)=E_{\text {out }, 1} / E_{i, 1}$, and the so-called drop-port response, $H_{21}(z)=E_{o u t, 2} / E_{i, 1}$, are calculated to be as follows

$$
\begin{aligned}
& H_{11}(z)=\frac{c_{1}-\gamma c_{2} e^{-j \varphi} z^{-1}}{1-c_{1} c_{2} \gamma e^{-j \varphi} z^{-1}}, \\
& H_{21}(z)=-\frac{s_{1} s_{2} \sqrt{\gamma e^{-j \varphi} z^{-1}}}{1-c_{1} c_{2} \gamma e^{-j \varphi} z^{-1}},
\end{aligned}
$$

where $c_{1,2}=\sqrt{1-\kappa_{1,2}}$ and $s_{1,2}=\sqrt{\kappa_{1,2}}$ are the through and cross field coefficients for the lower coupler and the upper coupler, being $\kappa$ the power coupling coefficient. Propagation and bend losses are incorporated in the parameter $\gamma$. The phase responses, considering $\varphi=0$, are calculated as

$$
\begin{aligned}
& \Phi\left(H_{11}(\Omega)\right)=\arctan \left[\frac{\left(c_{2} \gamma-c_{1}^{2} c_{2} \gamma\right) \sin (\Omega)}{c_{1}+c_{1} c_{2}^{2} \gamma^{2}-\left(c_{2} \gamma+c_{1}^{2} c_{2} \gamma\right) \cos (\Omega)}\right], \\
& \Phi\left(H_{21}(\Omega)\right)=\arctan \left[\tan \left(\frac{\Omega}{2}\right) \frac{c_{1} c_{2} \gamma+1}{c_{1} c_{2} \gamma-1}\right] .
\end{aligned}
$$

The offset of the resonance frequency of the RR can be adjusted by the additional phase shift, $\varphi$. The typical power transmission and phase response for the through-port and cross-port are depicted in Figure 3.10(a) and (b), as well as the zero-pole diagram on Figure 3.10(c).

Besides the frequency response or the $z$-transform, a ring resonator can be described by certain figure of merits, such as FSR, finesse, $\mathcal{F}$, and the quality factor $Q$. The FSR is the distance between two resonances peaks and is given in (3.1), where the $T_{\text {delay }}$ in the case of the ring resonator corresponds to the roundtrip time. Other two related parameters are the finesse, $\mathcal{F}$, defined as the ratio of the FSR to the width of a resonance for a specific wavelength, $F W H M$

$$
\mathcal{F}=\frac{F S R}{F W H M}
$$

and the quality factor, $Q$, defined as the ratio of the resonance frequency, $f_{0}$ to the width of a resonance for a specific wavelength, $F W H M$

$$
Q=\frac{f_{0}}{F W H M}
$$

The ring resonator can be described alternatively via the impulse reponse, as done for the AMZI in section 3.3.0.3. The response for the through-port of the ring resonator, $H_{21}(n)$, is shown in Figure 3.11. Note that unlike the AMZI, which has a finite impulse response, the optical ring resonator, because of the feed-back structure, has an infinite number impulse response output signals, and it can therefore be considered an infinite impulse response (IIR) filter. 


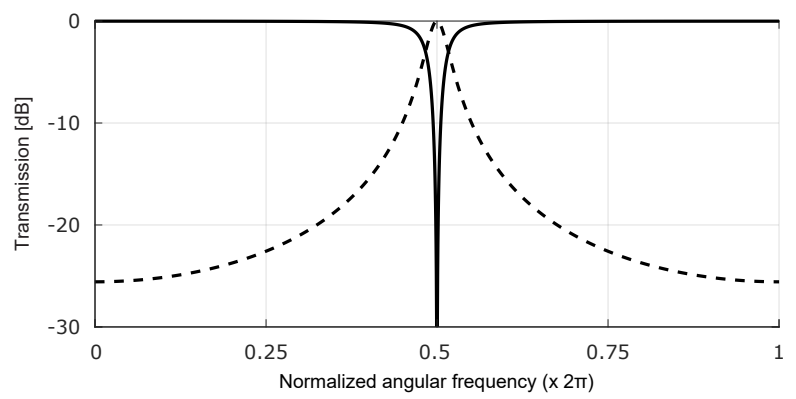

(a)

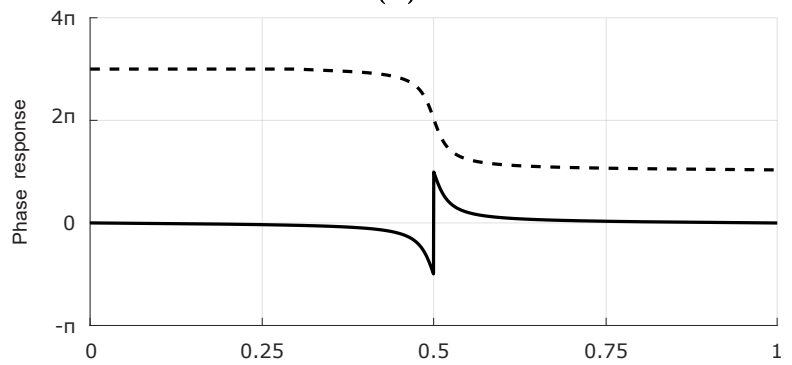

(b)

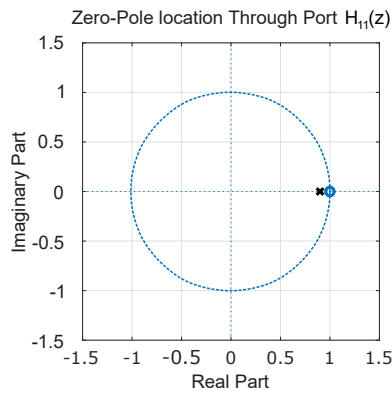

Zero-Pole location Drop Port $\mathrm{H}_{12}(\mathrm{z})$

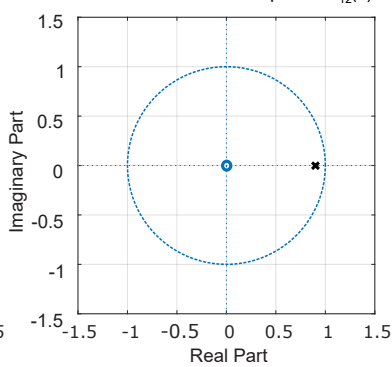

(c)

Figure 3.10: (a) Transmission response of a single ring resonator in ideal lossless case; (b) Phase response of a single ring resonator in ideal lossless. (c) Zero-pole diagram for a single ring resonator. (a), (b) and (c) are drawn for $\kappa_{1}=\kappa_{2}=0.1$ in the ideal lossless case. The solid line corresponds to the through-port, while the dotted line corresponds to the drop-port. In the zer-pole diagram the cross refers to the pole location, while the circle refers to a zero location. 


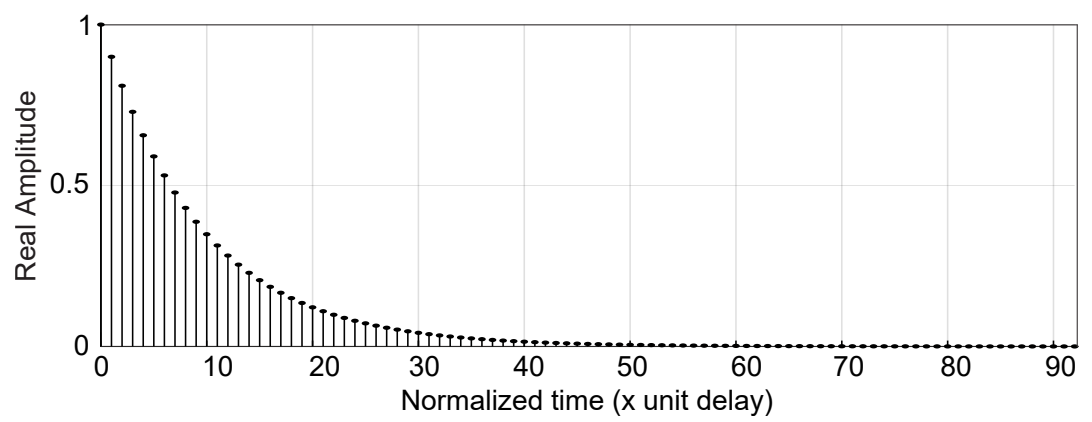

Figure 3.11: Impulse response of the through port of the ring resonator, $H_{21}(n)$.

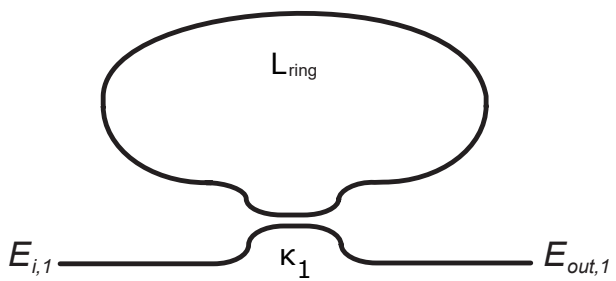

Figure 3.12: Schematic representation of a ring resonator as used in the OBFN

The ring resonator introduced here will be extensively used in the systems presented in this thesis, as building block for complex filters. One example is given in chapter chapter 5 , where eight RRs are coupled to each other to create a so-called coupled resonator optical waveguide (CROW) filter [22]. Another possibility of using such a building block is given in chapter 6 , where a somehow simpler version of the single ring resonator is used, as a building block for the optical beam-forming network (OBFN). A schematic representation of a RR, as used in the OBFN is given in Figure 3.12. Comparing Figure 3.12 with Figure 3.9, one can notice that the first is obtained from the second when taking the power coupling coefficient as $k_{2}=0$. The $z$-transform and the phase reponse can be calculated from (3.19) and (3.21) taking $c_{2}=1$. Such a resonator, or more often several cascaded RRs, are used in an OBFN because of the capability of manipulating the phase and consequently the group delay of a signal propagating through the RR. Figure 3.12 shows the normalized group delay response of the $\mathrm{RR}$ for different values of the power coupling coefficients. The normalized group delay can be calculated inserting (3.21) into (3.10), and it is expressed as

$$
\tau_{11}^{\prime}(\Omega)=\frac{\gamma c_{1} \cos (\Omega)-\gamma^{2} c_{1}}{1-2 \gamma c_{1} \cos (\Omega)+\gamma^{2} c_{1}}+\frac{\gamma^{2}-\gamma c_{1} \cos (\Omega)}{c_{1}^{2}-2 \gamma c_{1} \cos (\Omega)+\gamma^{2}} .
$$




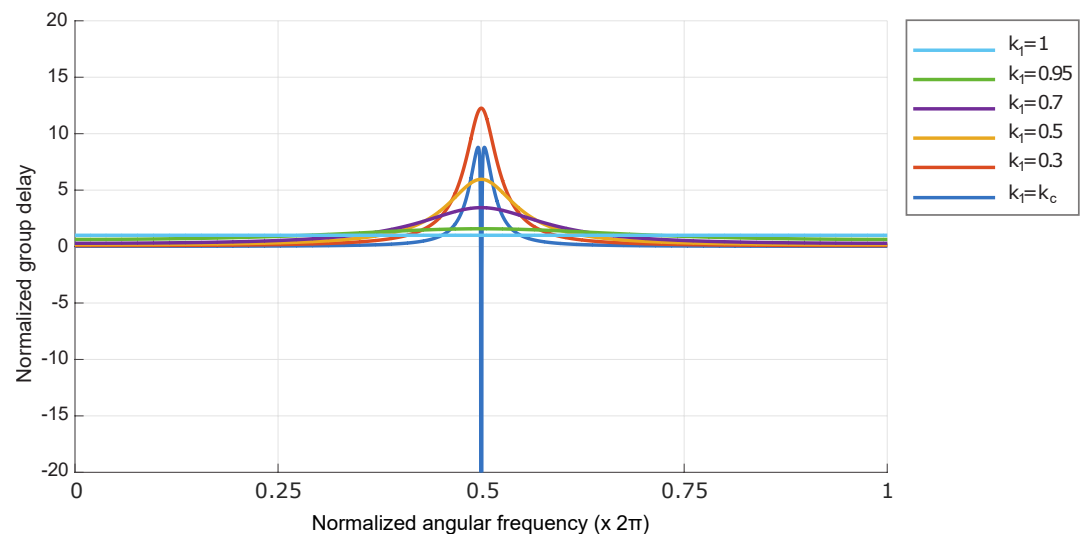

Figure 3.13: Group delay response of the through-port of a single ring resonator at different values of $\kappa_{1}$

The normalized group delay is constantly equal to one when $\kappa_{1}=1$. In this case the light is coupled to the RR and after exactly one roundtrip is coupled back to the output waveguide. As the power coupling coefficient is decreased to below unity, the group delay increases assuming a bell-shaped form centered at the resonance frequency until the so-called "critical coupling" value, $\kappa_{c}=1-\gamma^{2}$. Below this value, the group delay becomes partly negative within the resonance [23]. For the applications developed in this thesis is desirable to keep $\kappa_{1}$ in the range $\left\langle\kappa_{c}, 1\right]$ in order to have a positive delay.

Another important aspect that has to be taken into account is the bandwidth over which the group delay response is approximately constant. Figure 3.13 clearly shows that when the normalized group delay increases, the width of the resonance decreases. This occurs because the area under the group delay curve represents the total phase shift of the RR which is $2 \pi$ for one FSR. To increase the total phase shift as well, a combination of RRs can be used, which can achieve a certain almost constant group delay over a wider operational bandwidth [24]. Thus, having a single ring is simpler to realize and control, but might form a limit when used for wide-band applications. We will see in Chapter 6 how to cope with this limitation.

\subsubsection{Asymmetric Mach-Zehnder interferometer with two arms loaded with a ring resonator}

A combination of the basic elements described in the previous sections can provide devices that implement more complex MWP functionalities. In our systems an AMZI loaded with two ring resonators has been extensively used, to implement what we call an optical sideband filter (OSBF) as, for example, in chapter 6 . The name is due to the fact that it is used to achieve single-sideband 


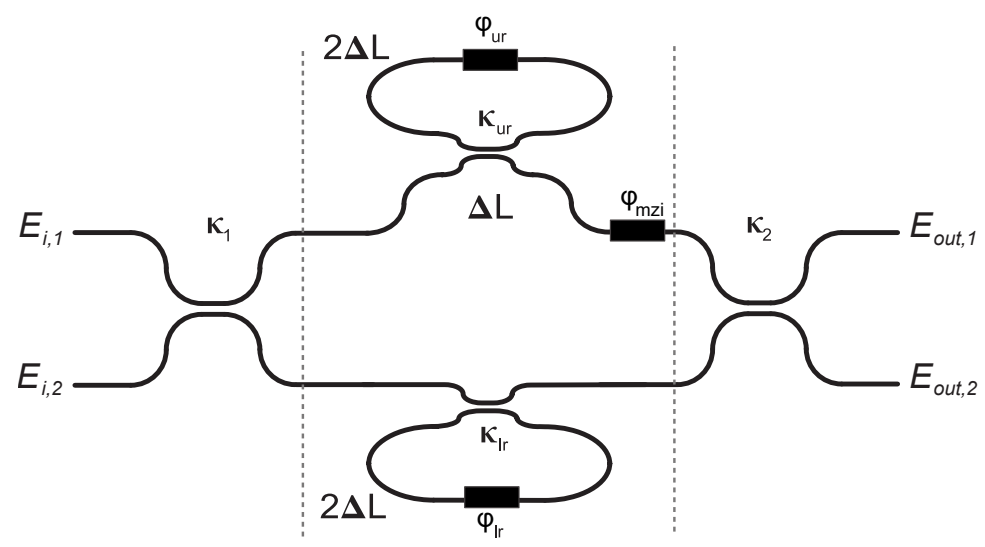

Figure 3.14: Schematic representation of an AMZI loaded with two ring resonators

(SSB) modulation. A closer look at the characteristics of this MWP filter is shown in Figure 3.14. The structure consists of an AMZI with a RR in each arm, where the differential path of the AMZI, $\Delta L$, sets the FSR of the filter and the length of the RRs, $L_{r}$, is chosen such that $L_{r}=2 \Delta L$. The role of the rings is to modify in a non-linear way the phase response of the two branches of the AMZI. The $z$-transform and from it the transfer function of the OSBF can be derived considering the transfer matrices of the three sections highlighted in Figure 3.14.

$$
\begin{aligned}
H(z) & =\eta\left[\begin{array}{cc}
c_{2} & -j s_{2} \\
-j s_{2} & c_{2}
\end{array}\right]\left[\begin{array}{cc}
H_{u r}(z) \gamma e^{-j \varphi_{m z i}} z^{-1} & 0 \\
0 & H_{l r}(z)
\end{array}\right]\left[\begin{array}{cc}
c_{1} & -j s_{1} \\
-j s_{1} & c_{1}
\end{array}\right] \\
& =\left[\begin{array}{ll}
H_{11}(z) & H_{12}(z) \\
H_{21}(z) & H_{22}(z)
\end{array}\right]
\end{aligned}
$$

where

$$
\begin{aligned}
& H_{u r}(z)=\frac{c_{u r}-\gamma^{2} e^{-j \varphi_{u r}} z^{-2}}{1-c_{u r} \gamma^{2} e^{-j \varphi_{u r}} z^{-2}} \\
& H_{l r}(z)=\frac{c_{l r}-\gamma^{2} e^{-j \varphi_{l r}} z^{-2}}{1-c_{l r} \gamma^{2} e^{-j \varphi_{l r}} z^{-2}}
\end{aligned}
$$

and where $c_{n}=\sqrt{1-\kappa_{n}}$ is the field coefficient for the $n$-th directional or tunable coupler and $\kappa_{n}$ is the relative power coupling coefficient, $\gamma$ is the amplitude transmission coefficient for an optical path equal to $\Delta L$, and $\eta$ takes into account the common path amplitude transmission and the corresponding phase shift. The through-port $z$-transform, $H_{11}(z)$, and the cross-port $z$-transform, 
$H_{12(z)}$, are express as follows

$$
H_{11}(z)=\frac{n_{t 0}+n_{t 1} z^{-1}+n_{t 2} z^{-2}+n_{t 3} z^{-3}+n_{t 4} z^{-4}+n_{t 5} z^{-5}}{d_{0}+d_{1} z^{-1}+d_{2} z^{-2}+d_{3} z^{-3}+d_{4} z^{-4}}
$$

and

$$
H_{12}(z)=-j \frac{n_{c 0}+n_{c 1} z^{-1}+n_{c 2} z^{-2}+n_{c 3} z^{-3}+n_{c 4} z^{-4}+n_{c 5} z^{-5}}{d_{0}+d_{1} z^{-1}+d_{2} z^{-2}+d_{3} z^{-3}+d_{4} z^{-4}} .
$$

The coefficients of the numerators of the two $z$-transforms are given by

$$
\begin{aligned}
& n_{t 0}=-s_{1} s_{2} c_{l r} \\
& n_{t 1}=c_{1} c_{2} c_{u r} e^{-j \varphi_{m z i}} \\
& n_{t 2}=s_{1} s_{2} \gamma^{2} e^{-j \varphi_{l r}}+s_{1} s_{2} c_{u r} c_{l r} \gamma^{2} e^{-j \varphi_{u r}} \\
& n_{t 3}=c_{1} c_{2} c_{u r} c_{l r} \gamma^{2} e^{-j\left(\varphi_{l r}+\varphi_{m z i}\right)}+c_{1} c_{2} \gamma^{2} e^{-j\left(\varphi_{u r}+\varphi_{m z i}\right)} \\
& n_{t 4}=-s_{1} s_{2} c_{u r} \gamma^{4} e^{-j\left(\varphi_{l r}+\varphi_{u r}\right)} \\
& n_{t 5}=c_{1} c_{2} c_{l r} \gamma^{4} e^{-j\left(\varphi_{l r}+\varphi_{u r}+\varphi_{m z i}\right)} \\
& n_{c 0}=c_{1} s_{2} c_{l r} \\
& n_{c 1}=s_{1} c_{2} e^{-j \varphi_{m z i}} \\
& n_{c 2}=-\left(c_{1} s_{2} \gamma^{2} e^{-j \varphi_{l r}}+c_{1} s_{2} c_{l r} c_{u r} \gamma^{2} e^{-j \varphi_{u r}}\right) \\
& n_{c 3}=-\left(s_{1} c_{2} c_{l r} \gamma^{2} e^{-j\left(\varphi_{l r}+\varphi_{m z i}\right)}+s_{1} c_{2} \gamma^{2} e^{-j\left(\varphi_{u r}+\varphi_{m z i}\right)}\right) \\
& n_{c 4}=c_{1} s_{2} \gamma^{4} e^{-j\left(\varphi_{l r}+\varphi_{u r}\right)} \\
& n_{c 5}=s_{1} c_{2} c_{l r} \gamma^{4} e^{-j\left(\varphi_{l r}+\varphi_{u r}+\varphi_{m z i}\right)} .
\end{aligned}
$$

The coefficients of the denominator are equal for both the $z$-transforms, and are expressed as follows

$$
\begin{aligned}
& d_{0}=1 \\
& d_{1}=0 \\
& d_{2}=-\left(c_{u r} \gamma^{2} e^{-j \varphi_{u r}}+c_{l r} \gamma^{2} e^{-j \varphi_{l r}}\right) \\
& d_{3}=0 \\
& d_{4}=c_{u r} c_{l r} \gamma^{4} e^{-j\left(\varphi_{l r}+\varphi_{u r}\right)} .
\end{aligned}
$$

Note that the other through- $\left(H_{22}() z\right)$ and cross- $\left(H_{21}(z)\right)$ port responses are the reverse polynomia of (3.29) and (3.30), respectively.

Such a filter is a $5^{\text {th }}$ order filter since both the $z$-transforms have five zeros and four poles (one pole is trivial). When the power coupling coefficients and phases of the filter are properly chosen, the transmission responses resemble the ones from a well-known digital filter, which is the IIR type II Chebyshev filter. This filter features flat pass-bands, equal-ripple stop-bands and steep 


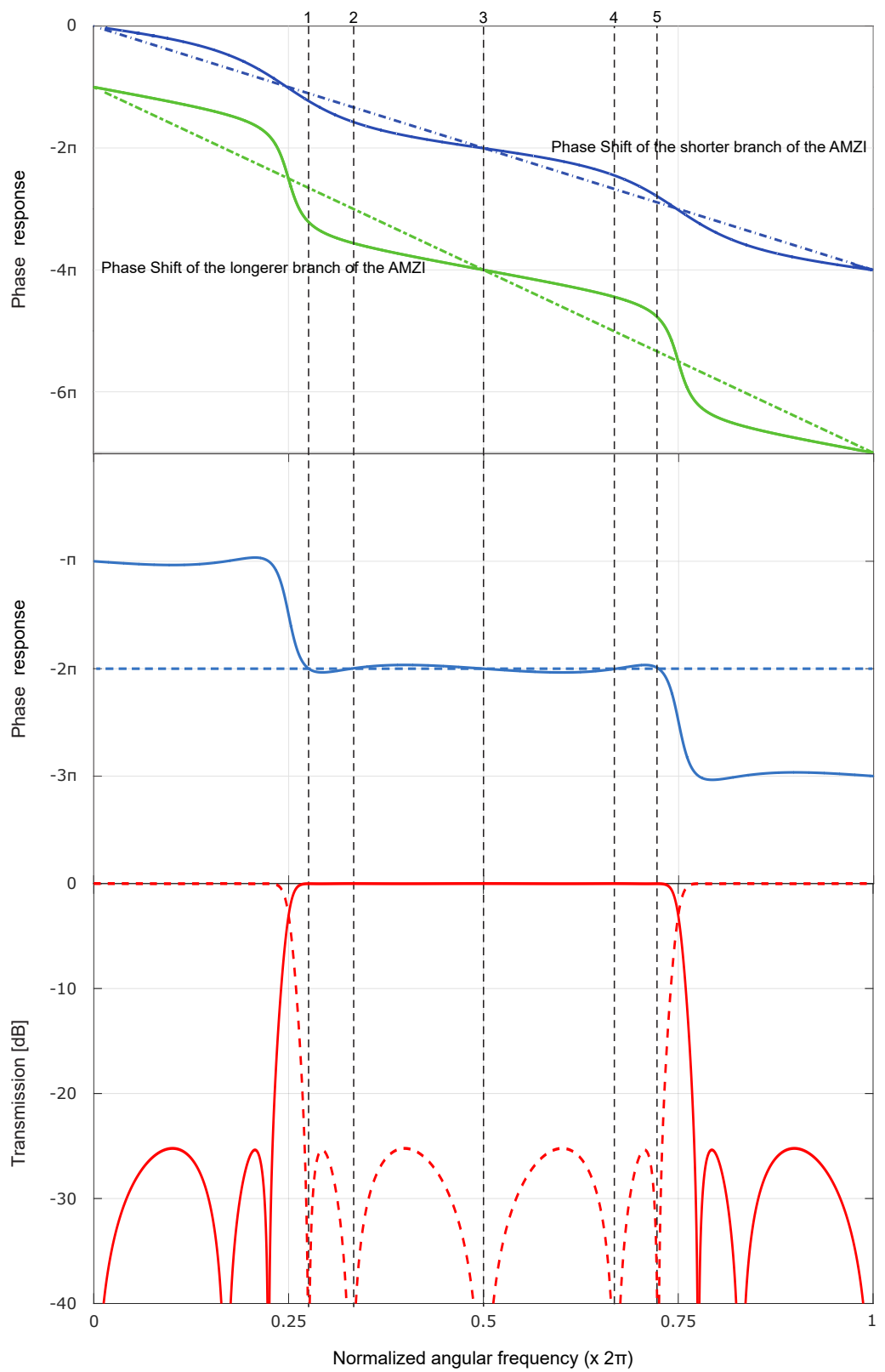

Figure 3.15: The top graph shows the phase response of the individual branches of the AMZI (solid lines) compared with linear phases which would produce the same overall phase shift (dotted line). The second graph gives the phase difference between the phase of the two branches is plotted. Last, the power transmission for through- (dotted line) and cross- (solid line) ports is shown, highlighting the location of the five zeros on the through-port response. 


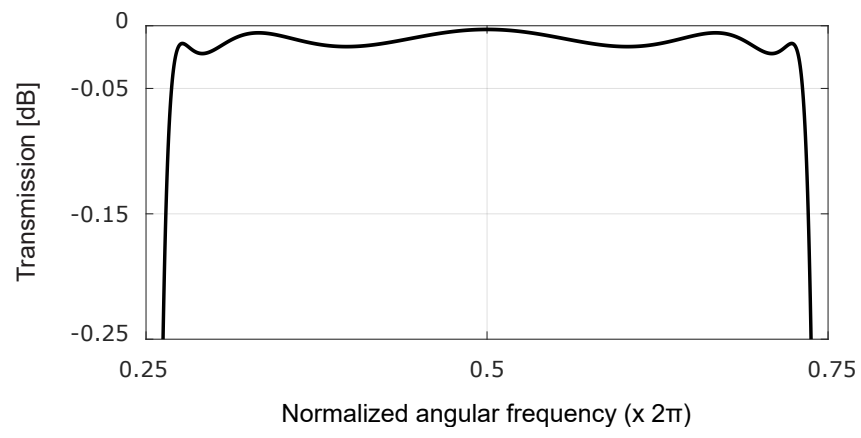

Figure 3.16: Zoom in of the transmission response for the passband, where the ripple caused by the five zeros in the $z$-transform is visible

transitions. Since the rings are added to both arms and the length of each ring is twice the length of the differential path of the AMZI, the phase shift for each arm will increase by $4 \pi$ in one period. Therefore for the short arm the overall phase shift in one period will be $4 \pi$, while for the long arm it will be $6 \pi$.

In Figure 3.15 the phase shifts for the long and short branches of the AMZI when the rings are fully coupled $\left(\kappa_{\text {ring }}=1\right)$ (solid lines) are compared to the case of proper setting of the coupling coefficients for the rings, such that the phase difference between the branches near the center of passbands and stopbands results in an approximately constant zero or $\pi$, as shown in the second plot of Figure 3.15. A zoom-in of the passband is given in Figure 3.17, where the ripple caused by the presence of the five zeros is visible. It has to be noticed that the value of the power coupling coefficients and the phases is very important, since a small change in one of this parameters drastically influences the transfer function. Figure 3.17 shows the impact of a $10 \%$ detuning for the phase of one ring $\left(\varphi_{u r}\right)$ on the transmission response. This is just an example, and ou calculations show that a change in any parameter will affect the transmission as well as the phase response of the filter so much that the filtering functionality might be compromised. In our case, those parameters can be actively adjusted after fabrication. 


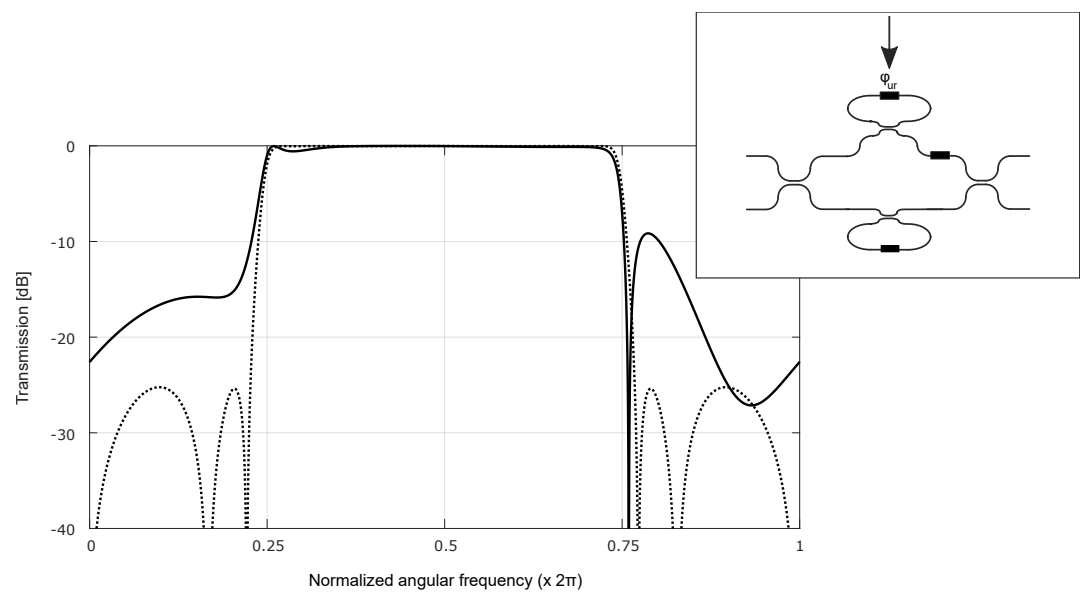

Figure 3.17: Transmission response of the cross-port of the AMZI loaded with two rings. The dotted line shows the ideal response of the filter, the solid line the transmission repsonse caused by a $10 \%$ detuning of the phase of the upper ring, $\varphi_{u r}$ 


\section{CHAPTER 4}

ANTENNA ARRAY THEORY

\subsection{Introduction}

An antenna is a device to convert guided electromagnetic waves, usually in the $\mathrm{RF}$ domain, into radiated (freely propagating) waves and vice versa [25]. The antenna design has to ensure that this conversion process is efficient. This means that the antenna should radiate as much power from the transmitter into a desired direction or that it collects as much power from a certain direction. In Figure 4.1 an example is shown where two antennas form a wireless link, one antenna sending out information encoded in RF waves and one for receiving the transmitted waves for decoding. Different figures of merit can be used to characterized an antenna. Here we present the parameters that are most commonly used for characterization.

A typical figure of merit for a transmitter is the effective isotropic radiated power (EIRP), which can be thought of as the amount of power that a perfectly isotropic antenna would need to radiate in order to achieve the same radiated power of the considered antenna in the direction of maximum gain,

$$
E I R P=P_{T} G_{T}[W],
$$

where $P_{T}$ is the input power fed into the transmitting antenna and $G_{T}$ is the so-called gain factor of the transmitting antenna. The latter is the factor by which the power transmitted into the desired direction is larger than with isotropic transmission from a non-directional antenna. Similarly, the antenna at the receiving side is characterized by a certain gain $G_{R}$. Assuming that the receiving wave has a power density $S\left[\frac{W}{m^{2}}\right]$, the received power can be 


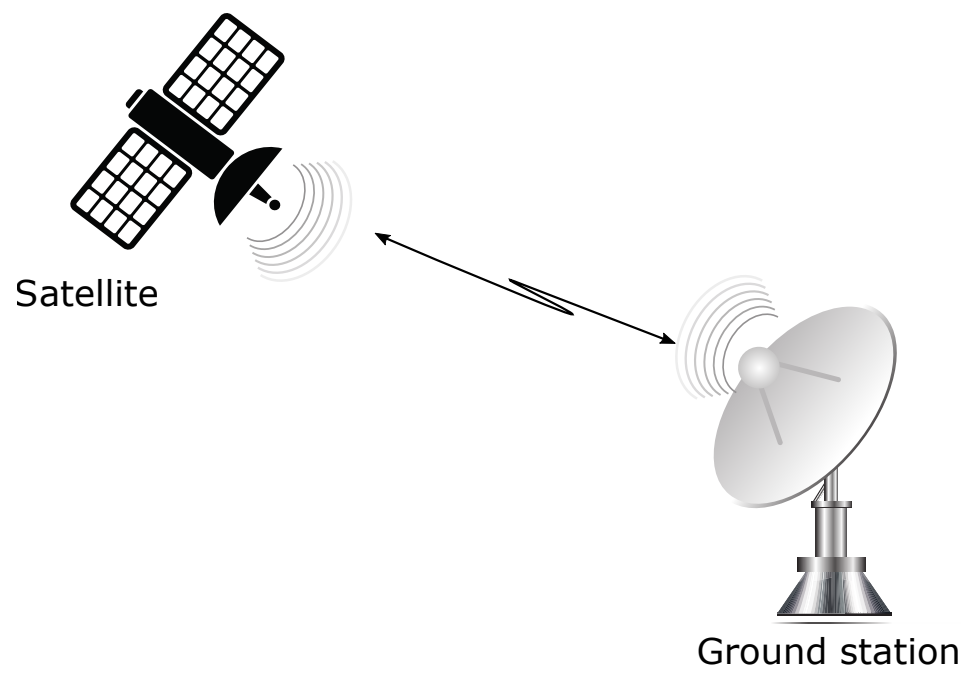

Figure 4.1: Example of a satellite communication link

expressed as

$$
P_{R}=A_{e} S[W],
$$

where $A_{e}\left[\mathrm{~m}^{2}\right]$ is the effective aperture of the antenna. For some antennas such as horn or dish antennas, the effective aperture might be close to the geometric area of the antenna projected onto the direction of reception. Nevertheless, the concept of an effective aperture can be applied to any kind of antenna, also if the effective aperture is much larger than the physical size of the antenna. As can be derived from the diffraction properties of electromagnetic waves, there is an approximate relation between the antenna gain and the effective aperture:

$$
G=\frac{4 \pi A_{e}}{\lambda^{2}},
$$

where $\lambda$ is the wavelength. In general the gain changes with direction and when the antenna is properly designed and aligned, it will develop a maximum gain and this gain is maximum in the direction of the receiver. For large distances, the transmitted power and the received power are approximately related via the well-known Friis transmission formula:

$$
P_{R}=P_{T} G_{T} G_{R}\left(\frac{\lambda}{4 \pi R}\right)^{2}=\operatorname{EIRPG}_{R}\left(\frac{\lambda}{4 \pi R}\right)^{2}[W],
$$

where $R$ is the distance between the transmitter and the receiver, $G_{T}$ and $G_{R}$ are the gain factors of the transmitting antenna and the receiving antenna respectively. (4.4) shows that an efficient link requires, for a fixed frequency 
and transmitted power, that the antenna design is optimum in terms of the antenna's gain parameters. From (4.3), the bigger the size of the antenna area compared to the wavelength $\left(A_{e} / \lambda^{2}\right)$, the higher is the gain. It can therefore be concluded that, to improve the reception of a signal, the antenna has to be large. This is an important conclusion to be used in the design of the MWP OBFN systems which will be fully described in the next chapters. Such systems are based on the concept of an antenna array, which is a form of what can be considered a large antenna, which is why antenna arrays are mostly used to increase the overall gain. A detailed analysis of antenna arrays is beyond the scope of this thesis and the reader can refer to [26]. Nevertheless, we will analyze the most important characteristics to understand how MWP OBFN systems, presented in chapter 6 and chapter 7 can enhance the existing array antennas.

An antenna array is a collection of individual radiating elements arranged in certain spatial configurations with respect to one another, for instance in a linear, planar or spherical arrangement, depending on what kind of directional coverage is required by the application [27]. The radiating elements are connected to a receiver or transmitter, depending on the kind of antenna, i.e., transmitting or receiving antenna, by a feeding network, whose role is to combine or split the signals keeping specific phase relationship. Such network is known as beamforming network. When an antenna array is used in a receiver, the RF signals at the individual elements have to be combined having the proper phase, to enhance signals received from the desired direction and cancel signals from other undesired directions. When instead an antenna array is used in a transmitter, the signals fed to the radiating elements have to have a certain mutual phase relationship in order to have constructive superposition of the radiated power in certain directions. In both cases, altering the phase relationship between the radiating elements means changing the direction at which reception or transmission of the antenna are maximum. In other words, the radiation pattern of the antenna, which briefly is the gain response of the antenna as a function of the angle (see section 4.2), is steerable. Usually the required manipulation of the phase relationship of the radiating elements is obtained by electronic control of the feeding network.

There are some clear and significant advantages of using antenna arrays over other conventional, mechanically controlled types of large antennas. First of all, wide-angle scanning without physically moving the antenna is possible [28] as well as adaptive beamforming [29]. Second, multiple independent beam directions can be simultaneously formed from a single aperture for a more efficient usage of power and bandwidth.

It is important to discern between two different possible kinds of antennas which are array-structured: phased-array antennas and timed-array antennas. In order to properly illustrate the difference between these two types of antennas, it is essential to clearly distinguish between the terms phase and time. For 


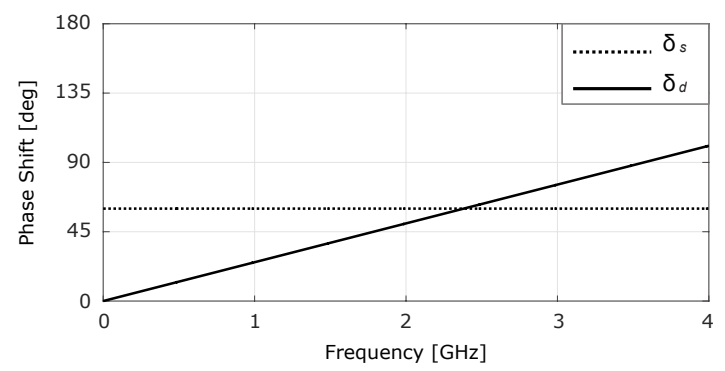

Figure 4.2: Difference between phase shifter (dotted line) and time delay (solid line) frequency behavior

this purpose, let us consider a sinusoidal signal

$$
s(t)=\sin \left[2 \pi f t+2 \pi f T_{d}+\delta_{s}\right]=\sin \left[2 \pi f\left(t+T_{d}\right)+\delta_{s}\right],
$$

where $t[s]$ is time, $T_{d}[s]$ is a time delay, whereas $\delta_{s}$ is a phase shift and has units of degrees or radians. Note that a given time delay can be uniquely (unambiguously) converted into a phase shift, using the relation

$$
\delta_{d}=2 \pi f T_{d}
$$

However, a given phase shift, because it is defined only modulo $2 \pi$, cannot be uniquely associated with a certain delay time. Instead, a phase shift corresponds to an infinite number of different time delays, as expressed by

$$
T_{s}=\frac{\delta_{s}}{2 \pi f}+n 2 \pi,
$$

and the individual values in this set of time delays depends on the frequency, $f$, to which one addresses the phase shift. This non-reciprocal relation between phase shift and time delay generates major problems when dealing with wideband signals. The consequence of this non-reciprocal relation can be appreciated when the terms in the argument of the sine function in (4.5) are evaluated with repect to frequency, namely the phase shift $\delta_{d}$, due to the time delay, $T_{d}$, as in (4.6) and the phase shift $\delta_{s}$, graphically shown in Figure 4.2. The first, $\delta_{d}$, depicted with solid line, is linearly related to the frequency, whereas the latter, $\delta_{s}$, in the dotted line, is simply a constant offset in the argument, independent of the frequency. This difference in dependence versus frequency, leads to a difference in array performance when the operating frequency changes with respect to the nominal center frequency for which the antenna system has been designed for, or also when wide-band signals, i.e., with multiple significant frequencies, are involved. 


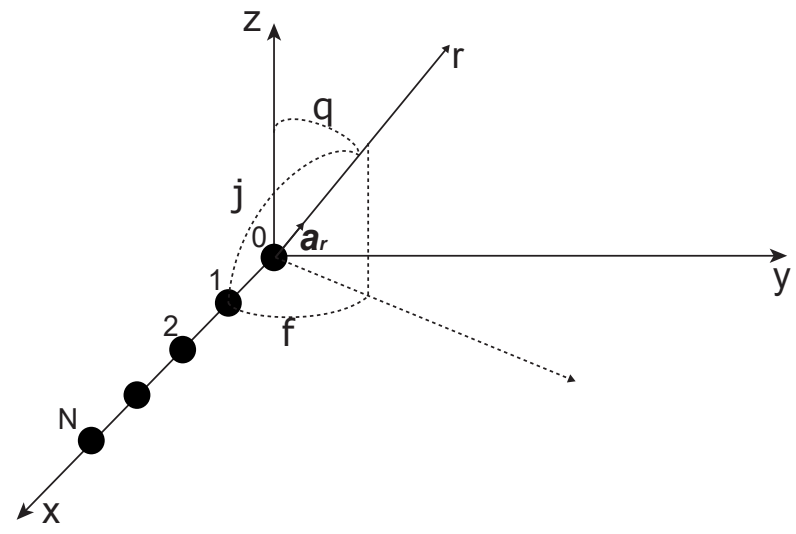

Figure 4.3: Representation of a linear antenna array with $\mathrm{N}+1$ identical antenna elements equally spaced

In a phase-shift-based array antenna, any different frequency around the nominal central frequency, will be steered with different deviations and this will change the direction of the maximum transmission or reception for different frequencies. This effect is known as beam-squint and will be further discussed in section 4.3. In time-delay-based array antenna, named true-time delay based, instead, all frequencies around the central frequency will be steered with the same deviation, and the antenna will not suffer any beam squint.

\subsection{Radiation pattern of array antennas}

In this section, a common parameter used for describing an antenna array is presented, namely the radiation pattern. Such parameter can be used to address the difference arised in the previous section between the phased array antennas and timed array antennas.

Let us introduce the antenna array depicted in Figure 4.3, where the dots indicates the locations of the $\mathrm{N}+1$ antenna elements. These antenna elements are equally spaced at a distance $d$ between each other along the $\mathrm{x}$ axis. Such configuration will be used as a reference for calculating the expressions of the radiation pattern in the case of phased-array antennas in section 4.3 and timedarray antennas in section 4.4 .

The normalized array radiation pattern of an antenna array in the far-field 
region $^{1}$ at the nominal central frequency, $f_{0}$, is given by

$$
\boldsymbol{E}(\theta, \phi)=\boldsymbol{f}(\theta, \phi) F(\theta, \phi)
$$

where $\boldsymbol{f}(\theta, \phi)$ is the radiation pattern for each element of the array expressed in spherical coordinates with $\theta$ and $\phi$ indicating elevation and azimuth angles respectively. For simplicity of description, it is assumed that $\boldsymbol{f}(\theta, \phi)$ is the same for all elements. $F(\theta, \phi)$ is the so-called array factor, that depends on the relative positioning of the elements and their mutual phasing or timing, and also on their relative radiation amplitude. The equation (4.8) is known as the principle of pattern multiplication. In order to compare the different kinds of antennas we will use the array factor, since it is the parameter that ultimately typifies and can be used to control the antenna array radiation pattern. As will be shown below, the array factor can be expressed as [30].

$$
F(\theta, \phi)=\sum_{i=0}^{N} C_{i} e^{j k \boldsymbol{a}_{r} \cdot \boldsymbol{r}_{i}},
$$

where $\boldsymbol{r}_{i}$ is the position of the $i$-th element with respect to the origin of the reference coordinate system and $\boldsymbol{a}_{\boldsymbol{r}}$ is the unit vector pointing towards the observation point under the assumption that the observation is done at large distance, i.e., in the far-field region of the radiation [31]. In (4.9), $k$ is the free-space wave number and it is expressed as

$$
k=\frac{2 \pi}{\lambda}=\frac{2 \pi f}{c},
$$

where $\lambda$ is the wavelength in free-space, $f$ is frequency and $c$ is the speed of light in vacuum. The array factor determines the shape of the radiation pattern and it can be controlled by changing the geometrical configuration of the array (given by $\boldsymbol{a}_{\boldsymbol{r}} \cdot \boldsymbol{r}_{i}$ ) and by properly setting the electrical excitation, in amplitude and phase, of the $i$-th element, represented by $C_{i}=\left|C_{i}\right| e^{j \delta_{i}}$. Thus, array antennas can have a reconfigurable radiation pattern by means of a beamforming network, that is used to set $\left|C_{i}\right|$ and $\delta_{i}$ for all the antenna elements such that the proper shaping of the radiation pattern is achieved.

Referring to Figure 4.3, the position vector of the $n$-th element is given by $\boldsymbol{r}_{n}=n d \hat{x}$, therefore, the scalar product in (4.9) becomes $\boldsymbol{a}_{\boldsymbol{r}} \cdot \boldsymbol{r}_{n}=n d \cos (\psi)=$ $n d \sin (\theta) \cos (\phi)$. The according array factor can be expressed as

$$
F(\psi)=F(\theta, \phi)=\sum_{n=0}^{N} C_{n} e^{j\left(\delta_{n}+n k d \cos (\psi)\right)}=\sum_{n=0}^{N} C_{n} e^{j\left(\delta_{n}+n k d \sin (\theta) \cos (\phi)\right)},
$$

\footnotetext{
${ }^{1}$ This region is known as the Fraunhofer region. Here, the radiation pattern does not depend on the distance. Moreover, in this region electric and magnetic fields are orthogonal to each other and the direction of propagation. Commonly the Fraunhofer region is considered to start from $R=\left(s D^{2}\right) / \lambda$, where $R$ is the distance from the antenna, $D$ is the maximum dimension of the antenna, and $\lambda$ is the wavelength.
} 
where $\psi$ indicates the angle between the $\mathrm{x}$ axis and the direction of the observer. This angle is also known as scanning angle. Suppose that the gain is to be maximized in the direction $\psi_{0}$, the coefficients $C_{n}$ have to be chosen to maximize $F\left(\Psi_{0}\right)$ as

$$
F\left(\psi_{0}\right)=\max _{\psi} F(\psi)=\sum_{n=0}^{N} C_{n} .
$$

In the next sections two different options to fulfill (4.12) will be presented, one for a phased array antenna and the second for a timed array antenna.

\subsection{Phased array antennas}

In the case of phased array antenna, the method for steering the beam direction in order to have maximum gain towards the desired direction, $\Psi_{0}$, is based on the use of phase shifters. Let us express the array factor of such phased array antenna, in order to evaluate the behaviour versus frequency.

The phase excitation at each element must be equal to $\delta_{n}$ expressed as

$$
\delta_{n}=-n k_{0} d \cos \left(\psi_{0}\right)=-n \frac{2 \pi d \cos \psi_{0}}{\lambda_{0}}
$$

where $k_{0}=2 \pi / \lambda_{0}$ for some frequency $f_{0}=c / \lambda_{0}$. Making the additional assumption of uniform amplitude excitation, that is $C_{n}=1$, and considering the phase excitation expressed in (4.13), the array factor in (4.11) becomes a geometrical series as

$$
\begin{aligned}
F(\psi) & =\sum_{n=0}^{N} e^{j n d\left(k \cos \psi-k_{0} \cos \psi_{0}\right)}= \\
& =\frac{1-e^{j(N+1) d\left(k \cos \psi-k_{0} \cos \psi_{0}\right)}}{1-e^{j d\left(k \cos \psi-k_{0} \cos \psi_{0}\right)}}= \\
& =e^{j \frac{N}{2} d\left(k \cos \psi-k_{0} \cos \psi_{0}\right)} \frac{\sin \left[\frac{N+1}{2} d\left(k \cos \psi-k_{0} \cos \psi_{0}\right)\right]}{\sin \left[\frac{d\left(k \cos \psi-k_{0} \cos \psi_{0}\right)}{2}\right]},
\end{aligned}
$$

By applying the substitution

$$
\begin{aligned}
u & =k d \cos (\psi), \\
u_{0} & =k_{0} d \cos \left(\psi_{0}\right),
\end{aligned}
$$

the magnitude of the array factor normalized to its peak value is expressed as

$$
F(u)=\frac{\sin \left[\frac{(N+1)}{2}\left(u-u_{0}\right)\right]}{\sin \left[\frac{\left(u-u_{0}\right)}{2}\right]} .
$$




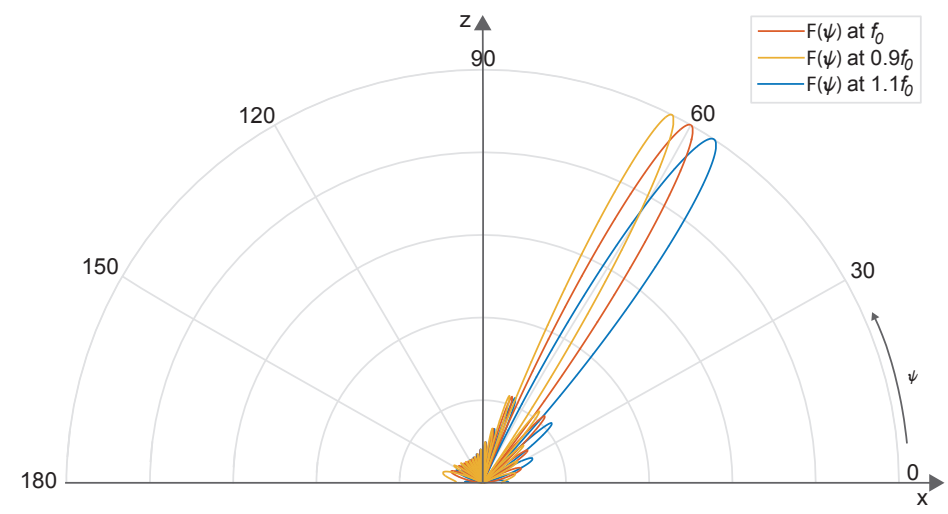

Figure 4.4: Radiation pattern as a function of $\psi$ for a 16-elements $\lambda / 2$-spaced linear array. The nominal pointing direction is $\psi_{0}=60^{\circ}$ at a center frequency of $1 \mathrm{GHz}$. When changing the central frequency, the beam squints to $56.2^{\circ}$ at $1.1 \mathrm{GHz}$ and moves to $63^{\circ}$ at $0.9 \mathrm{GHz}$.

Inserting (4.10) in (4.15), the array factor in (4.16) becomes

$$
F(\psi, f)=\frac{\sin \left[\frac{(N+1) \pi d}{c}\left(f \cos \psi-f_{0} \cos \psi_{0}\right)\right]}{\sin \left[\frac{\pi d}{c}\left(f \cos \psi-f_{0} \cos \psi_{0}\right)\right]} .
$$

It is evident (see Figure 4.4) that a beam is formed and that its direction points, as desired, for $f_{0}$ into the $\psi_{0}$-direction. However, because $(4.17)$ also depends on $f$, as the frequency increases or decreases from $f_{0}$, the beam will move (squint) from the desired steering angle $\psi_{0}$ to a new angle, $\psi_{s}$,

$$
\cos \psi_{s}=\frac{f_{0}}{f} \cos \psi_{0},
$$

as depicted in Figure 4.4. This means that a constant phase shift over all frequencies causes a squint, that increases with the bandwidth of the received or transmitted radiation, which imposes severe limitations in terms of bandwidth. When wide-band signals have to be transmitted (or received) it is thus necessary to use a better method of feeding the antenna elements, namely using timedelayed array antennas.

\subsection{Timed array antennas}

Following the same approach and using the same assumptions as in section 4.3, let us now consider an antenna array based on time delays instead of on phase shifters to be used to steer the beam direction. Let us consider intuitively the case of a receiver, without loss of generality, since the resulting array factor can 


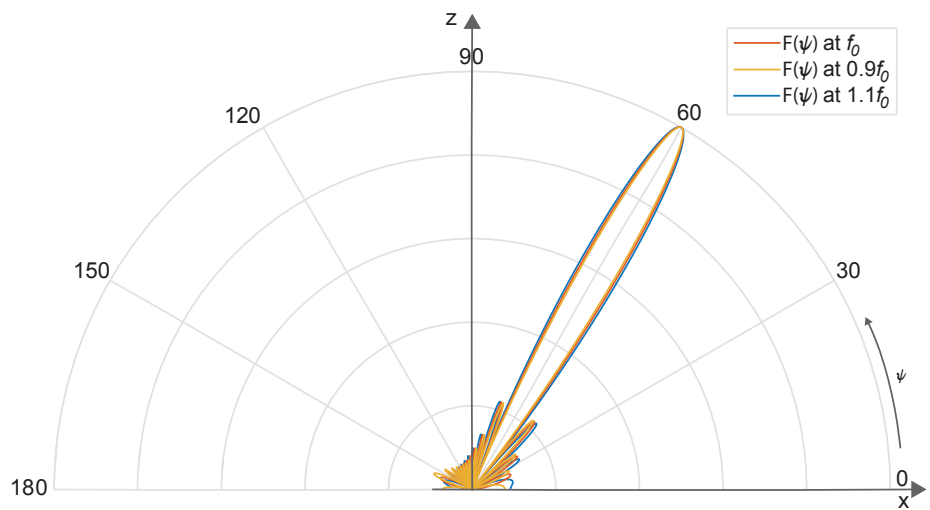

Figure 4.5: Radiation pattern as a function of $\psi$ for a 16-elements $\lambda / 2$-spaced linear array. The nominal pointing direction is $\psi_{0}=60^{\circ}$ at a center frequency of $1 \mathrm{GHz}$. When changing the central frequency, the beam pointing direction does not change.

be applied equally well to a receiving and a transmitting antenna, because of the reciprocity principle [32].

As the incoming signals arrive from a certain angle, $\psi_{0}$, to the antenna elements, then according to geometry in 4.3, the wave has to travel an additional distance equal to $d \cos \psi_{0}$ to arrive at each successive element. The time delay associated with the distance, assuming free-space is given by

$$
\Delta t=\frac{d \cos \psi_{0}}{c} .
$$

Using the conversion relation of (4.6), it is possible to express the time delay in terms of phase shift. Thus, the phase shift associated with $\Delta t$ is

$$
\delta_{n}=-2 \pi n f \Delta t=-\frac{2 \pi n d \cos \psi_{0}}{\lambda} .
$$

It is important to notice that the difference between (4.13) and (4.20) is the dependence of the second on $\lambda$. Substituting (4.20) in (4.11), the array factor for a timed array antenna can be expressed as follows

$$
F(\psi, f)=\frac{\sin \left[\frac{(N+1) \pi d f}{c}\left(\cos \psi-\cos \psi_{0}\right)\right]}{\sin \left[\frac{\pi d f}{c}\left(\cos \psi-\cos \psi_{0}\right)\right]} .
$$

A plot of the radiation pattern for the same three frequencies, as for the phased array radiation pattern, is given in Figure 4.5. The beams are now all pointing at the desired direction, $\psi_{0}=60^{\circ}$, independently of the frequency, and only the beamwidth varies slightly as the frequency varies. 
Timed array antennas are particularly important as context for this thesis since the systems developed in chapter 6 and chapter 7 are the processing cores of two beamforming networks, both based on time delays to achieve the desired radiation pattern. 


\section{CHAPTER 5 \\ COUPLED RING RESONATORS WAVEGUIDE FILTER}

\subsection{Introduction}

In this chapter, we present an MWP filter for RF signals. Filtering RF signals might be a trivial functionality to be achieved, however, realizing this functionality on an optical chip of small size, typically comprising only an area of a few square millimeters or centimeters, presents a significant challenge. The reason is that filtering or delaying $\mathrm{RF}$ signals after their up-conversion into the optical domain, requires optical filters that are extremely narrow-band and also well-controlled in spectral shape as compared to the optical carrier frequency. Typical, narrow filter bandwidths may amount to about $50 \mathrm{MHz}$, which is extremely small compared to the optical carrier frequency of about $200 \mathrm{THz}(1.5$ $\mu \mathrm{m}$ wavelength). This means that optical filters with huge quality factors, in the order of several millions, need to be realized. The latter requires to provide waveguide circuits with effective optical path lengths in the order of meters on a centimeter-sized photonic chip with low propagation loss, in order to retain a significant part of the optical power. In addition, the spectral shape of the transmission has to be configurable, e.g., to provide flat and dispersion-less passbands that can be set to any desired center frequency.

An important example where such functionalities are expected to form the key towards advanced performance are communications and data links between so-called smart satellites and within swarms of satellites. These systems would be based on performing onboard processing with flexible re-configurability. However, realizing these functions in the RF domain is problematic due to high energy consumption, large size and weight, sensitivity to electromagnetic 
interference, and also due to lack of flexibility, which is due to the need of handling predetermined data formats. A solution avoiding these disadvantages would be making use of an optically passive IMWP employing transparent integrated photonic circuits, e.g., as Input Multiplexers (IMUX) that accomplish partitioning of the satellite bandwidth into narrow sub-bands, an operation known as channelization. In multi-beam satellites, IMUX filters can also be integrated with routing networks and other signal processing functionalities.

However, integrated optical filters that can meet the highly challenging spectral and reconfigurational requirements, such as for channelization, have not been demonstrated so far. Specifically required is a filter bandwidth of 36 or $72 \mathrm{MHz}$ and a flat in-band response with small group delay variation $(<2 \mathrm{~ns})$ and low insertion loss $(<20 \mathrm{~dB})$. Another central requirement is a larger-than40-dB out-of-band rejection and a high steepness of the transmission function at the filter edges. This is quantitatively described with a small filter shape factor (SF), which is defined as the ratio of the -40-dB-bandwidth and the -6$\mathrm{dB}$-bandwidth. Desired values for SF lie in the order of $\mathrm{SF} \approx 2$, which is about 30 -times steeper than a Lorentzian filter $(\mathrm{SF}=58)$.

Here we demonstrate the first MWP filter suitable for broadband operation on board of a satellite due to its integrated photonic fabrication. The filter shows a flat-top passband of $72 \mathrm{MHz}$ width and a steepness given by $\mathrm{SF}=$ 1.9, an out-of-band rejection larger than $40 \mathrm{~dB}$ and a low ( $<2 \mathrm{~ns}$ ) group delay variation. The filter is fully reconfigurable in shape and center frequency. Passband flattening is obtained by forming a so-called CROW [22] with eight ring resonators, each having $21 \mathrm{~cm}$ optical length, with an adjustable resonance frequency and adjustable power coupling. The key to such performance is the waveguide platform based on stoichiometric $\mathrm{Si}_{3} \mathrm{~N}_{4} / \mathrm{SiO}_{2}$ fabricated via lowpressure chemical vapor deposition (LPCVD) [8], which offers an ideal combination of high index contrast, ultra-low propagation loss and high maturity for reliable fabrication of complex and reconfigurable circuitry on centimeter-sized chips. We note that MWP filters have been demonstrated by others as well, such as with cascaded silicon micro-ring resonators [33], stimulated Brillouin Scattering [34], [8], fiber Bragg gratings [35] or Mach-Zehnder interferometers [36]. These solutions are, however, relying on bulk components less suitable for application in satellites, require additional pump lasers to drive non-linear optical conversion, or they cannot match the spectral requirements in terms of passband bandwidth.

\subsection{Functional design of the coupled resonators optical waveguide filter}

A functional schematic of the fabricated filter is given in Figure 5.1. In the inset of Figure 5.1, a single stage waveguide schematic is highlighted, where 


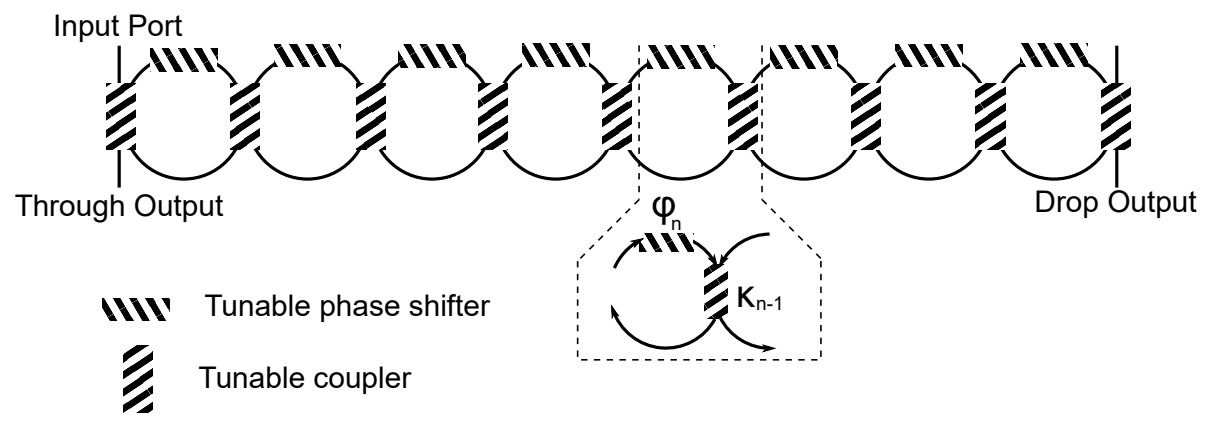

Figure 5.1: Functional schematic of the waveguide structure of the MWP filter

$\varphi_{n}$ denotes the phase shift of the $n$-th ring and $\kappa_{(n-1)}$ is the power coupling coefficient between the $n$ - 1 -th ring and the $n$-th ring. The spectral transmission functions of the circuit can be modeled via the $z$-transform, described in section 3.2, which yields the complex-valued field transfer function vs. light frequency within a FSR, in which the latter is mapped on the $2 \pi$-circumference of the unit circle in the complex z-plane. The $z$-transforms from the input to the drop output, and to the through output are obtained as

$$
\begin{aligned}
& H_{D R P}(z)=\frac{(-j)^{N+1} \sqrt{\sigma_{N}} e^{j \varphi_{T O T}} \gamma_{N} z^{-N}}{A_{N}(z)}, \\
& H_{T H R}(z)=\frac{B_{N}(z)}{A_{N}(z)} .
\end{aligned}
$$

In (5.1), $\mathrm{N}$ is the number of coupled RRs, $\sigma_{N}=\prod_{n=0}^{N} \kappa_{n}$ is the product of the power coupling coefficients, $\varphi_{T O T}=\sum_{n=1}^{N} \varphi_{n}$ is the total phase shift imposed by the resonators, and $\gamma$ is the ring amplitude transmission coefficient, which is determined by the waveguide loss. $A_{N}(z)$ and $B_{N}(z)$, in (5.2) are two $N$-th order polynomial in $z^{-1}$, with their coefficients depending on the values of $\kappa_{n}$ and $\varphi_{n}$ [37]. Factorizing the polynomial $A_{N}(z)$ into its roots, it is straightforward to see that $H_{D R P}(z)$ is a product solely of poles in the complex plane, while $H_{T H R}(z)$ is a product of both poles and zeros.

To demonstrate how to impose in the transfer functions (5.1) and (5.2) the desired spectral shape, with high steepness and rejection, we have calculated the settings of the coupling coefficients that provide a maximally flat peak in $H_{D R P}(z)$. Maximum flatness is obtained with a so-called Butterworth filter (here of $8^{\text {th }}$-order), and the according coefficients and phases were derived using the modified Levinson algorithm [37], with the coefficients displayed in Table 5.1. Looking at the values of the power coupling coefficients in the table, it is noticed that the ones in the center of the CROW are smaller with respect to the outer values. Deviations of any phase from $0^{\circ}$ lead to asymmetric spectra.

We note that the shown values provide the optimum transmission functions only for the ideal case of zero loss, while different values are expected depending 
Table 5.1: Optimum circuit parameters for a maximally flattened (Butterworth) microwave photonic filter realized as an $8^{\text {th }}$-order CROW calculated via [37]

\begin{tabular}{||c|c||}
\hline Parameter & Theoretical calue derived via [37] \\
\hline \hline$\kappa_{0}, \kappa_{8}$ & 0.343 \\
\hline$\kappa_{1}, \kappa_{7}$ & 0.025 \\
\hline$\kappa_{2}, \kappa_{6}$ & 0.011 \\
\hline$\kappa_{3}, \kappa_{5}$ & 0.009 \\
\hline$\kappa_{4}$ & 0.0085 \\
\hline$\phi_{1}$ to $\phi_{8}$ & $0^{\circ}$ \\
\hline
\end{tabular}

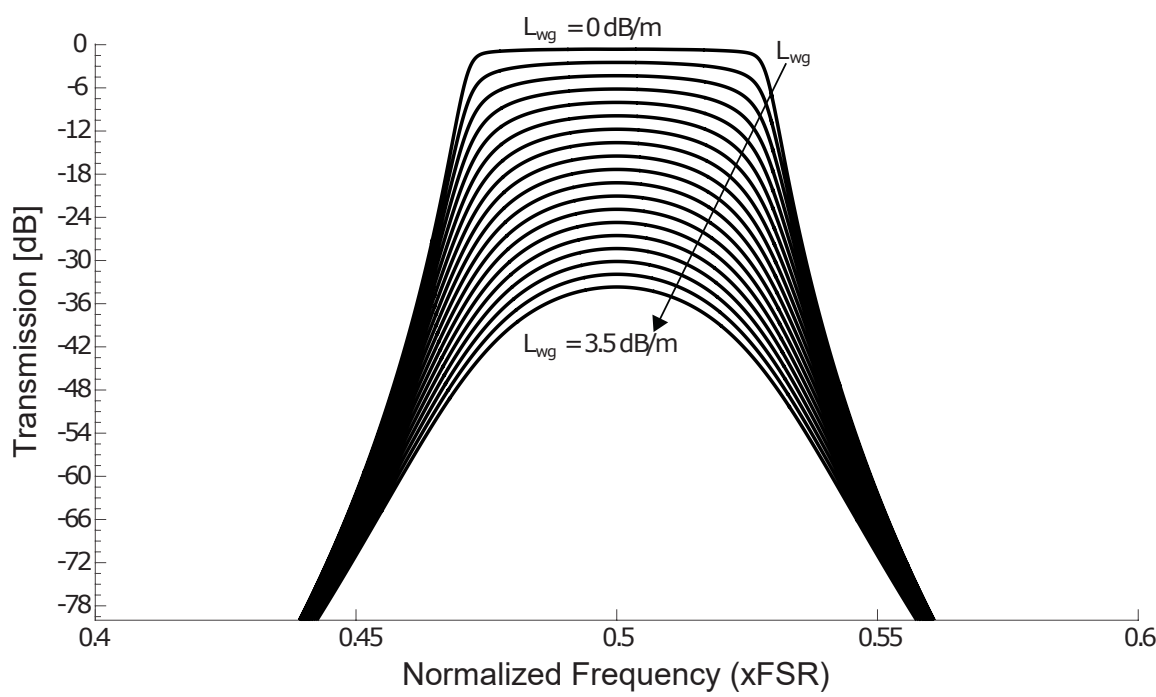

Figure 5.2: Power Transmission response for different values of waveguide loss

on the waveguide characteristics. The accordingly calculated power transmission to the drop output vs frequency in units of the FSR is shown in Figure 5.2, for various different values of the waveguide loss parameter. It can be seen the spectral shape deviates progressively from the desired flat shape (small SF) towards a rounded shape (high SF) if the waveguide loss parameter is increased, even if the assumed loss is extremely small $(<0.035 \mathrm{~dB} / \mathrm{cm})$. This limitation does not only affect the passband profile but also the group delay dispersion, as it is shown in Figure 5.3(a) and Figure 5.3(b). It can be seen, as the waveguide loss increases, that the $3 \mathrm{~dB}$ bandwidth becomes smaller through progressing curvature, whereas the group delay is least dispersive at some intermediate loss value. Similarly, the transmission band in the through output becomes rounded whereas intermediate losses yield the least dispersive shape. From the lowering of the peak transmission in the drop output one can see that the insertion loss 
- No Loss

$0.2 \mathrm{~dB} /$ roundtrip Loss

- $0.5 \mathrm{~dB} /$ roundtrip Loss
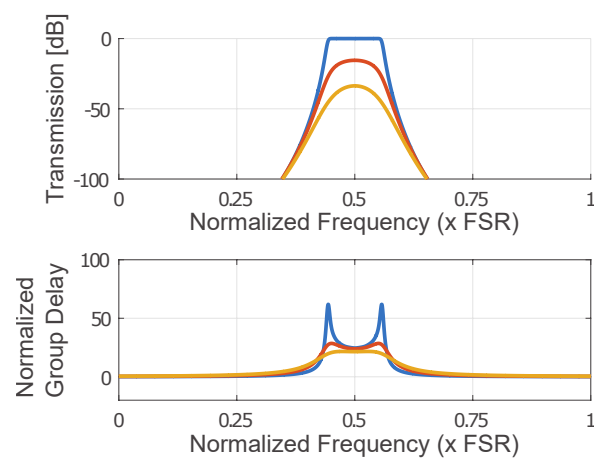

(a)
- No Loss

$0.2 \mathrm{~dB} /$ roundtrip Loss

- $0.5 \mathrm{~dB} /$ roundtrip Loss
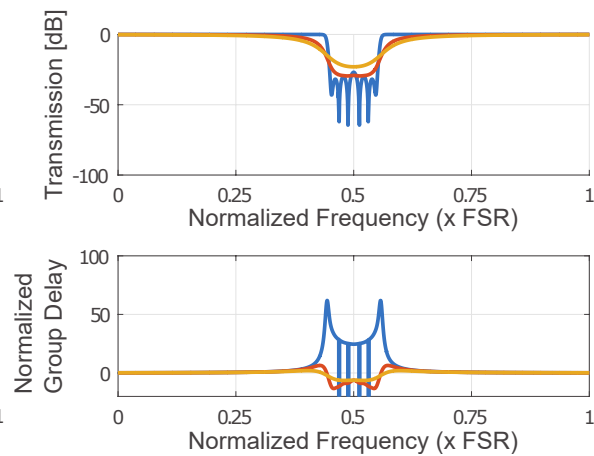

(b)

Figure 5.3: Calculated power transmission and normalized group delay for a) the Drop output and b) the Through output

increases noticeably with the waveguide loss parameter. We note that all of these calculations and considerations involve extremely low levels of waveguide loss, namely below $0.035 \mathrm{~dB} / \mathrm{cm}$. This underlines the central importance of selecting a waveguide platform that can offer such low loss and still allows to implement complex waveguide circuits on a small, chip-sized area.

\subsection{Fabrication and demonstration of the cou- pled resonators waveguide filter}

We have fabricated the circuit shown in Figure 5.1 using an advanced dielectric waveguide platform based on a combination of silicon nitride layer $\left(\mathrm{Si}_{3} \mathrm{~N}_{4}\right)$ as waveguide layer(s) and silica $\left(\mathrm{SiO}_{2}\right)$ as cladding layers [38], using a single-stripe geometry. A typical SEM picture of the single-stripe waveguide structure is given in Figure 5.4. The platform and particularly the single-stripe geometry was chosen because it allows extremely low propagation loss [9], because the circuit parameters can be electrically tuned, and because a high fabrication reliability can be achieved due to maturity of the platform. A full tunability of the device is obtained with resistive heater elements that control the power coupling coefficients and phase shifters.

The fabricated chip has been designed for an FSR of $1.4 \mathrm{GHz}$. A graphical impression of the fabricated chip is shown in Figure 5.5. The chip is bonded to a customized PCB connected to a flat cable and each heater is addressed by a custom developed control board via USB. The optical inputs and outputs are 


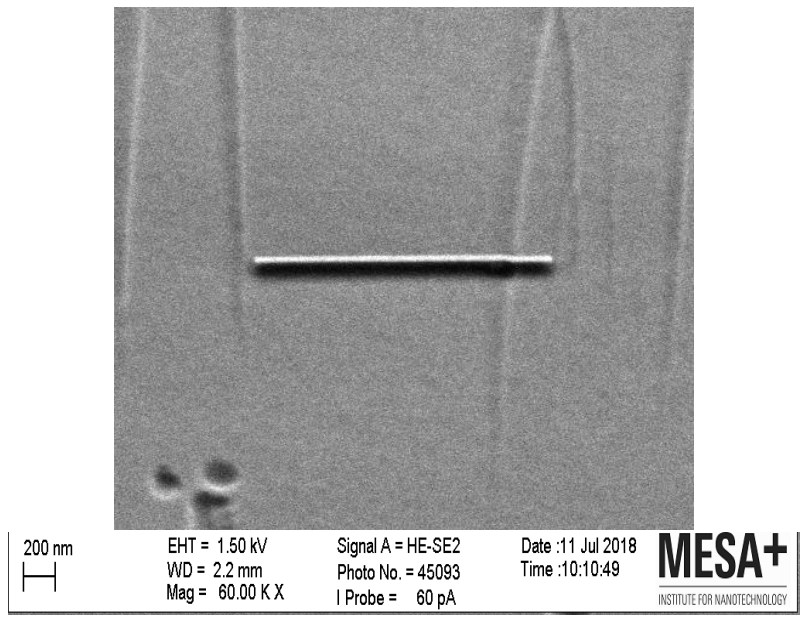

Figure 5.4: SEM picture of the cross-section of the single-stripe waveguide in TripleX

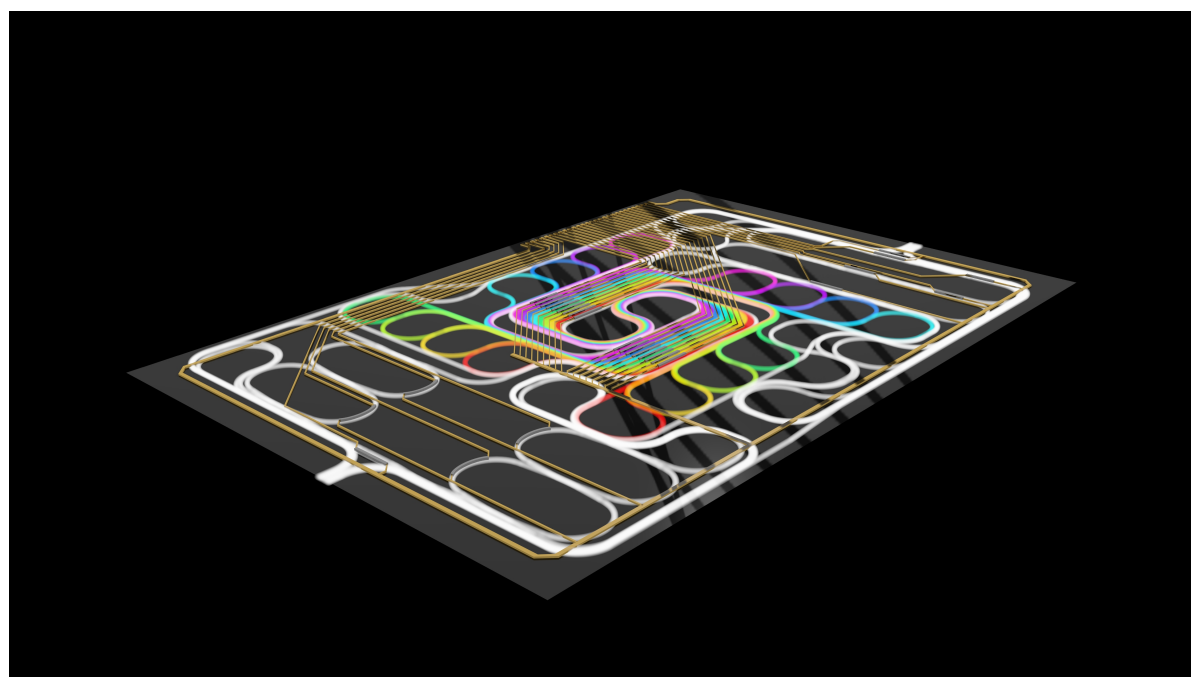

Figure 5.5: Graphical impression of the fabricated chip, where the waveguides are highlighted in different colors 


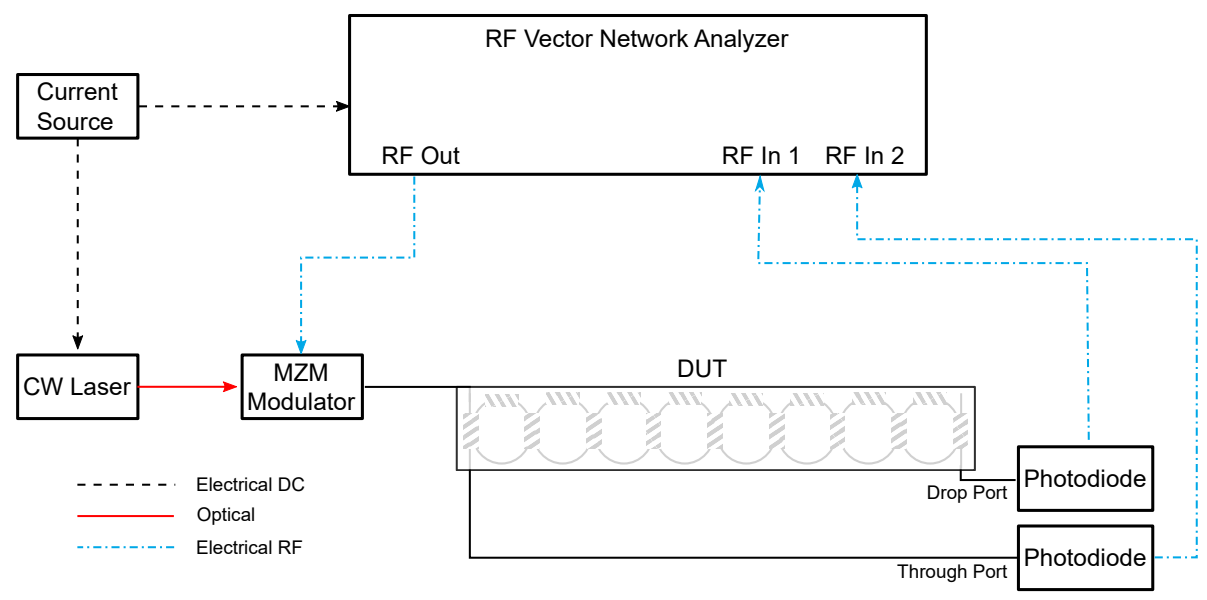

Figure 5.6: Scheme of the measurements setup

connected to fiber arrays. Using a straight test waveguide on the same chip, we measured a fiber to chip coupling loss of about $3 \mathrm{~dB} /$ facet.

In Figure 5.6 the experimental setup used for the characterization of the MWP filter is shown. A single-frequency laser (EM4-253-80-057, spectral bandwidth $1 \mathrm{MHz}$ ) is used as optical carrier and is intensity-modulated by a $10 \mathrm{MHz}$ signal from a vector network analyzer (VNA) (VNA, Agilent NA5230A PNA$\mathrm{L}$ ), before sending the light into the CROW filter. The driving current of the laser is increased linearly, causing a corresponding to a wavelength tuning to measure the frequency response across one or more FSRs. The outputs (drop and through) from the filter are detected by two photodiodes (Discovery Semiconductor DSC-R401 HG, bandwidth $20 \mathrm{GHz}$ ) and sent to the VNA for measuring power and phase of the transmitted signals vs light frequency. The transmission spectra were manually optimized to a best compromise of narrowest bandwidth, highest flatness and lowest group delay dispersion for the drop output. The accordingly required settings for the heater elements were found with separate experimental calibration of the circuit parameters of each ring resonator, before setting the parameters to the theoretically expected optimum values (see table 5.1). The adjustment of the phases was done by minimizing any spectral asymmetry in the transmission spectrum. Improvements in steepness, flatness and bandwidth narrowing were performed manually, although ultimately a computer control can be applied as well.

The measured power and group delay spectra after optimization are plotted in Figure 5.7(a) and (b). The measured width of the passband is $72 \mathrm{MHz}$, which is, to the best of our knowledge, the narrowest bandwidth obtained for an integrated MWP filter using only passive components. The measured maximum group delay variation in the passband is approximately $2 \mathrm{~ns}$. The measured filter transmission and delay are in excellent agreement with the 


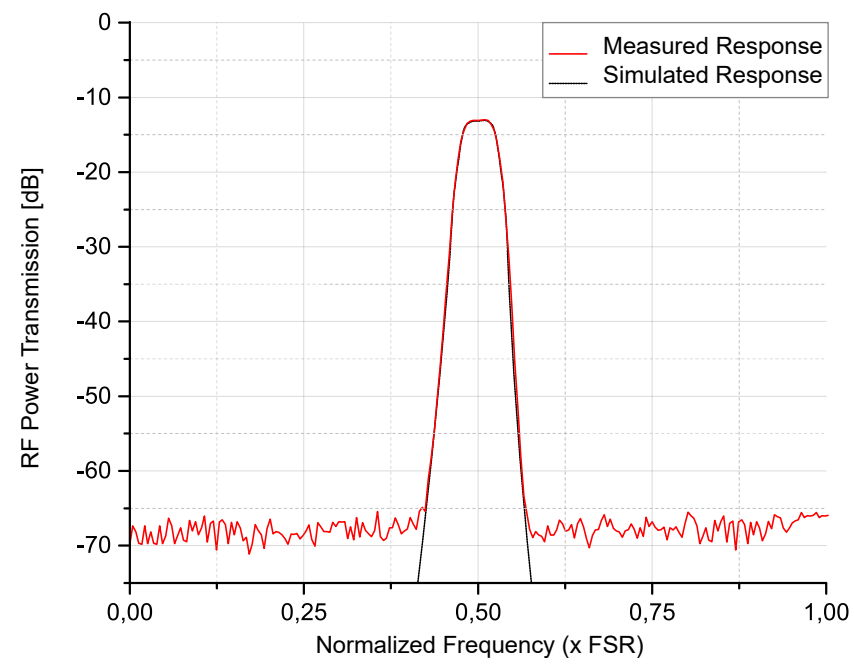

(a)

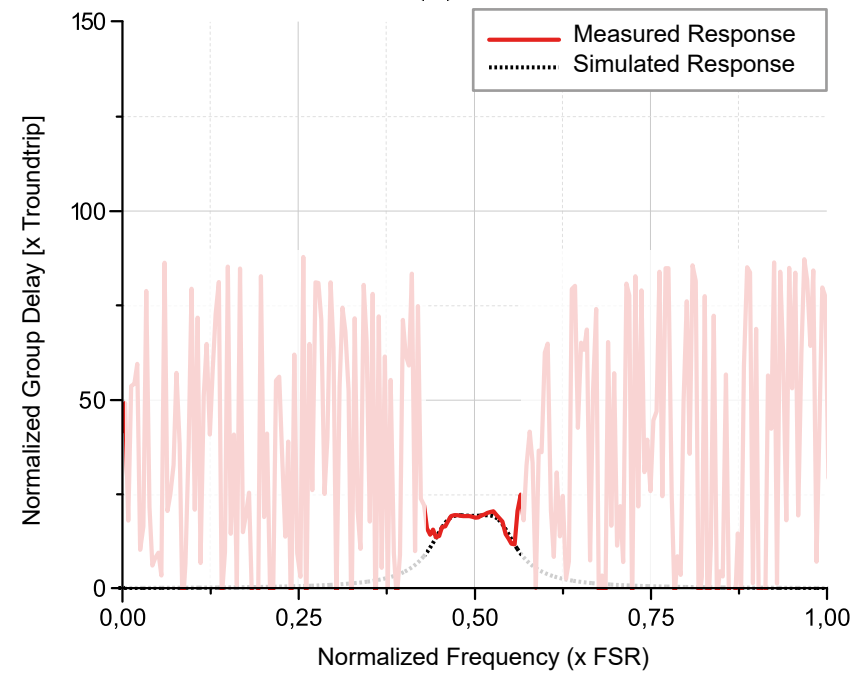

(b)

Figure 5.7: Measured and simulated a) RF transmission response and b) group delay of the Drop output of the fabricated CROW filter 


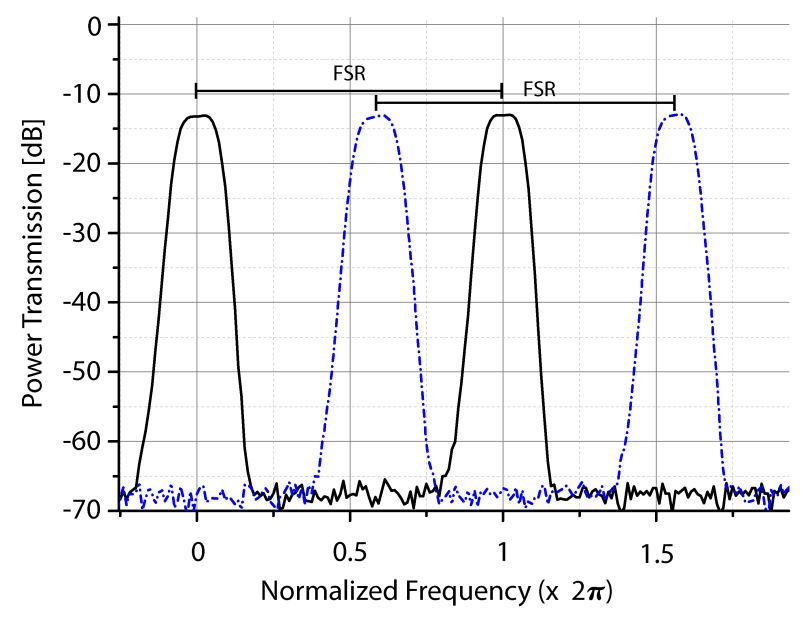

Figure 5.8: RF Power transmission response of the Drop output measured tuning the filter at two different center frequencies

theoretical model when using model parameters close to the ideal values. The measured shape factor of the filter, $\mathrm{SF}=1.93$, is rather close to the ultimate lossless limit $(\mathrm{SF}=1.34)$. The out-of-band rejection, particularly interesting when the filter is employed as IMUX to reject adjacent channels, is at least $51 \mathrm{~dB}$ (limited by the experimental noise floor of the equipment). The overall insertion loss of the filter is $13 \mathrm{~dB}$. From this value and the parameters in the theoretical curves in Figure 5.2, we estimate that the waveguide loss is as low as $0.014 \mathrm{~dB} / \mathrm{cm}(1.4 \mathrm{~dB} / \mathrm{m})$.

To demonstrate reconfigurability, we have tuned the transmission to an adjacent spectral interval by a larger distance that is about half of the FSR, in this case about $0.7 \mathrm{GHz}$. From Figure 5.8 can be seen that the shape, transmission and out-of-band rejection of the filter are reproduced with high fidelity. This feature allows an active change of the parameters in order to have the filter centered at any desired frequency within the FSR.

Also the transfer function to the through output was inspected. An example is shown in Figure 5.9, where the power transmission is displayed for the following three different settings of the optical couplers. The dotted blue curve is measured when setting the coupler $\kappa_{1}$ to zero (between the first ring and the second ring in Figure 5.1). In this case one obtains the transmission function of a simple ( $1^{\text {st }}$-order $)$ notch filter that can be tuned with the input coupler $\left(\kappa_{0}\right)$ between the bus waveguide and the first ring, as described in section 3.3.0.4. The measured full width at half maximum (FWH) for the notch filter is 14 $\mathrm{MHz}$, which corresponds to a finesse of about 100 from (3.23), confirming the waveguide loss value named above. Analogously, manipulating the coupler to 


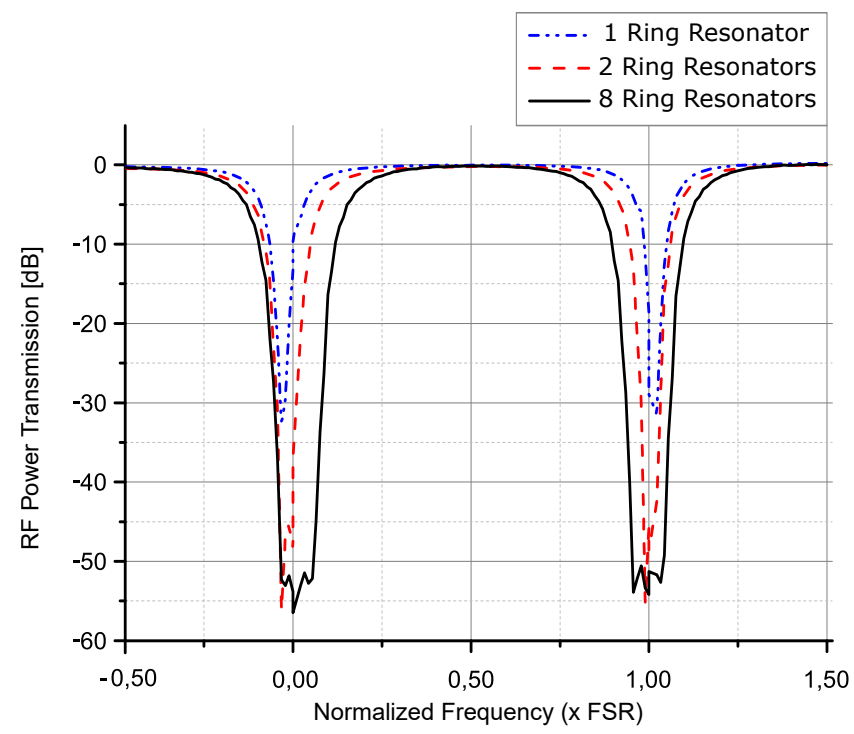

Figure 5.9: RF Power transmission response of the Through output measured involving a growing number of RRs

the second resonator generates a wider $\left(2^{\text {nd }}\right.$-order filter $)$ with similar slope at the filter edges, progressing towards an $8^{\text {th }}$-order filter.

\subsection{Conclusion}

We have demonstrated an integrated optical passband MWP filter based on a CROW structure comprising eight mutually coupled ring resonators. The filter has been fabricated using an ultra-low loss waveguide platform based on a $\mathrm{Si}_{3} \mathrm{~N}_{4}$ core in a $\mathrm{SiO}_{2}$ cladding. The filter shows a record narrow passband flattened bandwidth of $72 \mathrm{MHz}$ (filter shape factor 1.93) and an out-of-band rejection of at least $51 \mathrm{~dB}$. These values indicate a very low waveguide loss of around $0.014 \mathrm{~dB} / \mathrm{cm}$ and a large effective optical length in the order of several meters on a chip. The filter is fully tunable and reconfigurable in shape and center frequency. In combination with the through port output response of the same CROW filter it appears feasible to integrate multiple DMUX and MUX functionalities. The compact and rugged format and high energy efficiency of such microwave photonic processing devices appear suitable even for demanding applications, such as in smart satellite systems, and moreover it can be easily integrated with other functionalities, such as OBFN to provide a compact and robust system. 


\section{CHAPTER 6}

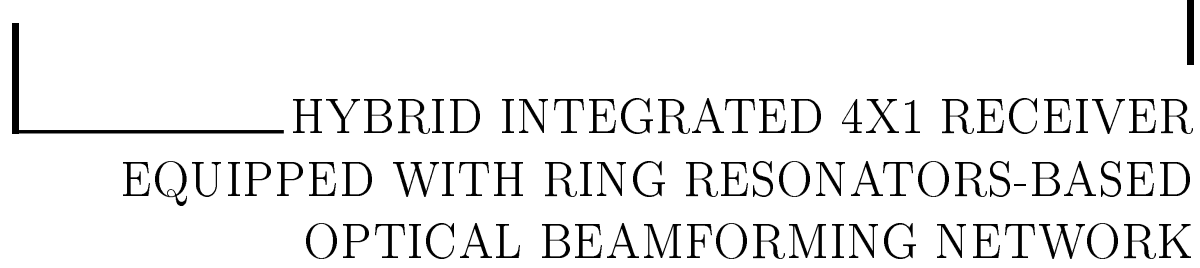

\subsection{Introduction}

In this chapter a hybrid integrated MWP OBFN system is presented, which is designed to be used at the receiving side of a satellite communication link. An MWP implementation of the OBFN using true-time delay (TTD) leads to significant advantages. Particularly, the system implemented here is based on ring resonators as the processing core. The choice of having an MWP system based on hybrid integration is mainly motivated by the required optical signal processing, namely the beamforming network, which must be characterized by extremely low-loss waveguide propagation since the ultimate goal is to have long delay lines in the order of several tens of centimeters. For this purpose, the waveguide technology based on $\mathrm{Si}_{3} \mathrm{~N}_{4} / \mathrm{SiO}_{2}$, namely TripleX ${ }^{\mathrm{TM}}$, has been selected as the platform for the OBFN system because of the combination of low propagation losses, as low as $0.1 \mathrm{~dB} / \mathrm{cm}$, and high refractive index contrast between core and cladding, the latter allowing small bend radii (of about 100 $\mu \mathrm{m}$ ) and thus a more compact layout. The waveguide technology used in this case is based on asymmetric double-stripe structure. A typical SEM picture of the cross-section of such a structure is given in Figure 6.1. Here, one can notice two rectangular waveguides with different widths, which are fabricated in silicon nitride $\left(\mathrm{Si}_{3} \mathrm{~N}_{4}\right)$ and surrounded by silicon oxide $\left(\mathrm{SiO}_{2}\right)$. More details about the waveguide technology can be found in [38].

Concerning the active devices ultimately needed to perform all the function- 


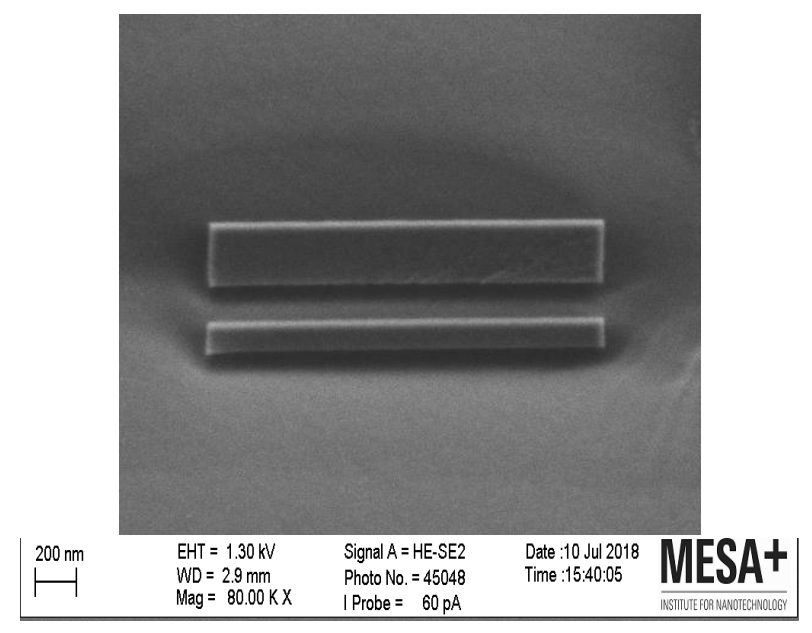

Figure 6.1: SEM picture of the cross-section of an asymmetric double-stripe waveguide in TripleX.

alities required to have a complete APL, namely light generation, modulation, and detection, the chosen active material is InP since, regarding integrated technologies, is the most efficient for performing the required active functionalities.

The hybrid integrated multichip system which will be analyzed here is a $4 \mathrm{x} 1$ receiver system, based on true-time delay OBFN for a linear antenna array consisting of four antenna element (AE)s. A picture of the assembled hybrid integrated microwave photonic $4 \times 1$ receiver is shown in Figure 6.2. The system comprises the TripleX chip, which is the biggest chip in the center of the picture, at which the InP chip is attached on the left side. The TripleX chip is then connected by wire-bonding to customized PCBs, used to drive the heating elements present on the chip. The PCB on the left-hand side is instead used for injecting the current to the gain diode, to apply RF modulation signals to the modulator and to collect RF signals from the photodiodes.

\subsection{Hybrid integrated laser}

The first necessary building block is a single-frequency light source, which will be used as optical carrier. In order to have an on-chip laser light with ultralow linewidth, the light source is implemented in the form of a hybrid diode laser as depicted in Figure 6.3, comprising a semiconductor gain diode for the generation and amplification of light and an external cavity for spectral control 


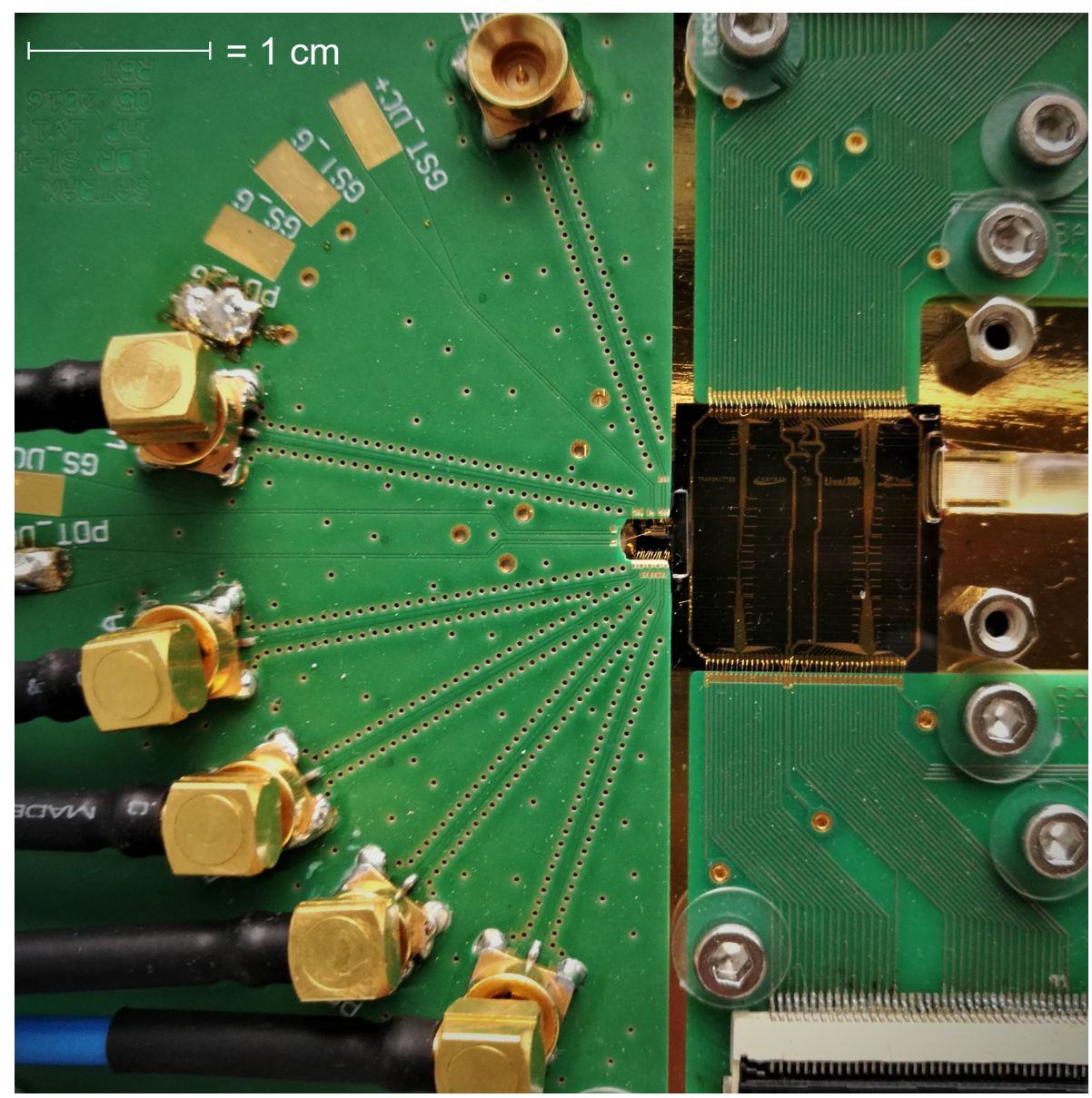

Figure 6.2: Picture of the hybrid integrated microwave photonic $4 x 1$ receiver 


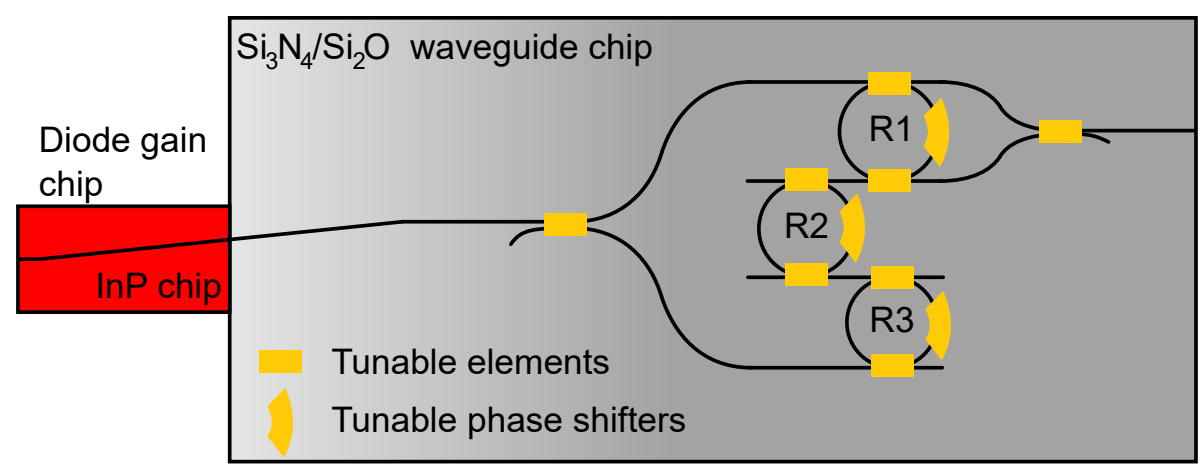

Figure 6.3: Schematic representation of the hybrid laser with an active gain section, the diode gain chip, coupled to a passive waveguide section, the $\mathrm{Si}_{3} \mathrm{~N}_{4} / \mathrm{SiO}_{2}$ waveguide chip, which comprises a ring resonators-based tunable reflector.

of the generated light.

The advantage of having a semiconductor-based laser is that it enables to generate coherent light directly with electrical pumping, which can provide a high electric-to-optical efficiency and an easy electronic control. Making use of an external cavity, that extends the photon lifetime and provides sharp and tunable spectral filtering, offers wider tuning range and a narrower spectral linewidth as compared with standard monolithic diode lasers.

The chosen semiconductor optical amplifier is based on InP that operates at around $1.55 \mathrm{\mu m}$, which is one of the wavelength used for telecom applications, while the optical feedback waveguide circuit that forms the external cavity arm has been fabricated using the $\mathrm{Si}_{3} \mathrm{~N}_{4} / \mathrm{SiO}_{2}$ waveguide platform. Hybrid semiconductor lasers based on this concept, called semiconductor-glass waveguide hybrid laser [39], can provide single-frequency oscillation with widest spectral coverage and record-narrow spectral linewidths from a few tens of $\mathrm{kHz}$ to even sub-kHz values [40]. Here, although the hybrid integrated laser is not further investigated in this thesis and rather adapted as an existing component, we give a brief description of its operational principle for completeness. The semiconductor diode laser amplifier is similar to a common semiconductor Fabry-Perot diode laser. For providing amplification in double-pass, the chip is equipped with a high-reflection coated back facet while the front facet is anti-reflection coated, with a sligthly tilted angle of the exit waveguide with regard to the surface normal. The external cavity consists of three ring resonators having slightly different radii (and thus also different free-spectral ranges) with respect to each other. The power coupled to each ring and the optical roundtrip phase in each ring can be adjusted by thermo-optical tuning. The consequence of this particular arrangement is a large overall FSR of the triplet of resonators, known as the Vernier effect. The power coupling coefficients to be selected as 


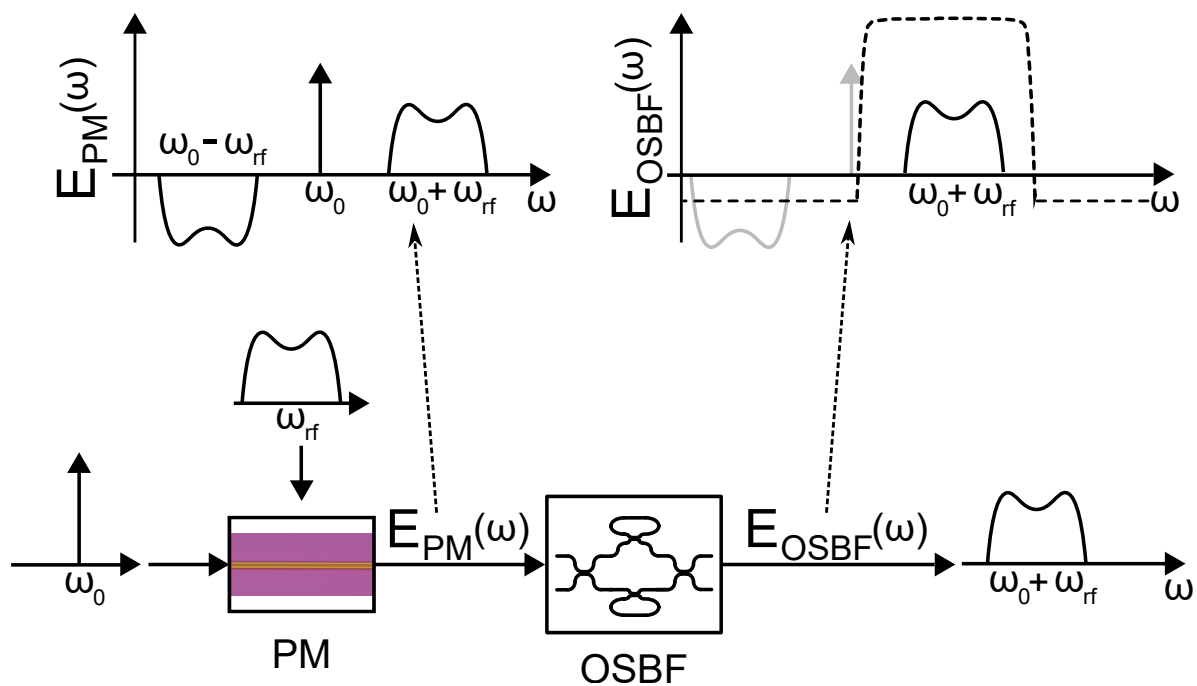

Figure 6.4: Schematic representation of single-sideband suppressed-carrier modulation achieved by optical spectral filtering. The spectra of the signals at different locations in the APL are illustrated to show the processing role of the phase modulator and the optical sideband filter

to limit oscillation to a single frequency within the amplifier gain bandwidth can be calculated via the method proposed in [41]. The described configuration ensures tunable single-mode operation where multiple roundtrips in the three resonators increases the effective cavity length to induce a narrow laser linewidth in the order of tens of $\mathrm{kHz}$ and a tunability range of more than 80 nanometers. For more details, one can refer to [41, 39].

\subsection{Optical single-sideband modulation and single-sideband filter}

When considering an MWP beamforming network, either working in transmission or reception, the information to be transmitted or received is present in the microwave RF domain. For processing in the optical domain it is thus necessary to translate them from the microwave RF domain into the optical domain using modulators for electrical-to-optical conversion and photodiodes as demodulators. In general, modulators form an essential building block of any APL, independently from particular processing functionality to be carried out. In the system presented here, having the choice between the two techniques described in section 2.1.2, we favored phase over intensity modulation due to the following benefits. Phase modulators can work under bias-free op- 
eration, a key advantage, since the bias drifting, eventually happening in the alternative, i.e., in intensity modulation based on MZMs, is eliminated [42]. Besides this, phase modulators can be implemented with a simpler waveguide structure which lowers the overall system complexity with smaller footprint. However, though a phase modulator simplifies the modulation process, it will also render the detection more elaborate. Namely, a phase-modulated signal possesses a constant envelope and power, and thus cannot be directly detected with a photodetector (PD). Instead, to recover the microwave signal, the phasemodulated signal needs to be converted such that it can be detected as power modulation. Different schemes to detect a phase-modulated signal have been proposed and they can be divided mainly into two broad categories: detection after optical spectral filtering [43], and interferometric detection [44].

The technique chosen in this work is based on optical spectral filtering. More specifically, the dual or multiple sideband spectrum generated by phase modulation is processed by a dedicated filter in order to have in the remaining spectrum only a single sideband present. Having SSB modulation is convenient from two points of view. The first is that the modulation is directly detectable as a power (intensity) modulation with a photodetector. The second is that the optical bandwidth to be processed by the OBFN can be relaxed with respect to double-sideband (DSB) modulation, as we will describe in section 6.4.

Figure 6.4 displays the working principle, on which the receiving system is based. The goal of the spectral filtering is to achieve single-sideband suppresedcarrier (SSB-SC) modulation by using an OSBF. Here, first the optical carrier is modulated by the phase modulator, denoted with PM. The spectrum after modulation, denoted with $E_{P M}(\omega)$, is shown as inset. The dedicated OSBF, after the modulator, processes the spectrum such that SSB-SC modulation is achieved. The spectrum after filtering, $E_{O S B F}(\omega)$, will consist of only one sideband, as observed in the inset, which can be either the upper or the lower one. In fact, setting the filter to pass only the upper sideband implies that the carrier is filtered out as well because the carrier and the other sideband are in the stopband of the filter, as shown in the inset. Note that the filter can be used also to achieve single-sideband full-carrier (SSB-FC), by shifting the frequency response.

\subsection{Optical beamforming networks}

The OBFN is the actual processing core of the IMWP system and it consists of delay elements and combiners. Here, the case of a receiving OBFN is described.

The schematic structure of the TTD receiver is shown in Figure 6.5. The AEs which collects the RF signals are shown on the left side of the figure. The signals from the AEs are fed into the modulators, denoted with $\mathrm{E} / \mathrm{O}$, for electrical-to-optical conversion. Here, the waveguides at which the optical 


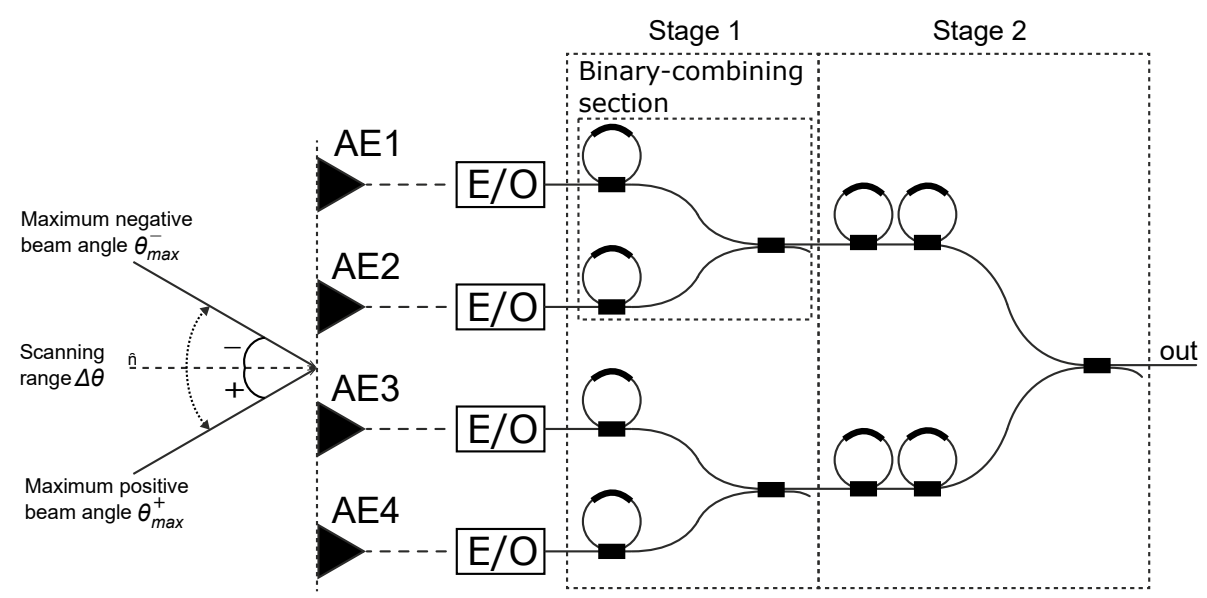

Figure 6.5: Schematic layout of a symmetrical binary-tree structured $4 \mathrm{x} 1$ receiving OBFN

carrier is injected are not shown. The modulated signals at optical frequencies enter the OBFN, where they are synchronized (brought into phase) and combined (superimposed). In this case, the OBFN has a symmetrical binarytree structure. Two stages can be identified, denoted with stage 1 and stage 2. Each stage consists of one or more binary-combining sections, one of them highlighted within stage 1 as surrounded by a dashed-line box in Figure 6.5. In each binary-combining section, two adjacent channels are first synchronized and then combined. The synchronization is achieved by using RRs as delay elements. The required number of rings depends ultimately on the angle of incidence of the RF signals on the AEs and the bandwidth. Having a symmetrical structure, with a delay element on both arms of each binary-combining section, enables to steer towards positive and negative beam angles. At each stage a new imaginary array of AEs is formed, where the number of elements is half while the inter-element distance is twice with respect to the previous stage. The combining process continues until the signals from all AEs are combined into a single output. For a given number of AEs, $M$ (being power of 2), the number of stages, $K$, can be calculated as $K=\log _{2} M$. The beam scanning angular range is defined symmetrical about the normal of the antenna plane: $\left[\theta_{\max }^{-}=-\Delta \theta / 2, \theta_{\max }^{+}=\Delta \theta / 2\right]$, as represented in Figure 6.5 and the upper and lower arms of each binary-combining stage have the same maximum time offset, considering that for positive angles, the lower arm has advanced-time offset when compared to the upper arm, while for negative angles it is the other way around. The maximum advanced-time offset of the $k$-th stage $M_{-} T_{k}$ can be calculated as

$$
M_{-} T_{k}=-\frac{\sin (\Delta \theta / 2) \cdot 2^{k-1} \cdot d}{c_{0}}
$$

The total number of RRs in the OBFN is the sum of the RRs of all binary- 
combining sections:

$$
N_{r r, t o t}=\sum_{k=1}^{K} \frac{M}{2^{k}} \cdot N_{k}
$$

Here $M / 2^{k}$ is the number of binary-combining sections per stage $k$ and $N_{k}$ is the number of RRs in a binary-combining section of stage $k$. The number of RRs per each arm of a binary-combining section, which is the same in each stage in a symmetrical structure, can be determined upon knowing the requirements for the system in terms of operating RF frequency, $f_{R F}$, antenna element spacing, $d$, the maximum beam steering angle, $\Delta \theta$, maximum allowed ripple in the phase, $\Delta \phi$, and the modulation scheme. In the case of SSB modulation, for instance, the number of RRs has to be such that the group delay response is approximately constant (within a certain specification) over the SSB bandwidth. Earlier research [24] has shown that, for a given phase ripple specification, the required number of RRs per delay element is roughly proportional to the product of required delay tuning range and the required optical bandwidth.

The calculation for the number of rings is irrespective of the FSR of the RRs, as long as the optical bandwidth to be processed is well below the FSR. In general, it is desirable to make the FSR as large as possible. As the FSR is inversely proportional to the circumference, the larger the FSR, the smaller the required total chip area. The upper bound for the FSR is ultimately given by the minimum bend radius allowed by the technology.

\subsection{Photodetection}

In order to keep the overall complexity of the system as low as possible, the OBFN is optimized to guarantee a constant delay response that covers only the remaining sideband bandwidth after the filtering to achieve SSB-SC modulation. In the receiver case, it is necessary to reinsert the unmodulated carrier prior to optical detection, as illustrated in Figure 6.6. For this purpose, the optical carrier is split-off before the modulation takes place, via controlling the value of $\kappa_{1}$. The unmodulated carrier will be available to be combined with the SSB-SC-modulated output signal of the OSBF by means of a second tunable coupler, with value of $\kappa_{2}$, just before the photo-detector, denoted with PD. To calculate the spectrum at the $\mathrm{PD}$, let us express the optical field at different locations, namely at $\mathrm{A}, \mathrm{B}$ and $\mathrm{C}$ in Figure 6.6. The carrier at point $\mathrm{A}$ to be re-inserted amounts to a certain fraction of the unmodulated carrier provided by the laser, and therefore it can be described by a complex bandpass signal $E_{c}$ with an optical angular frequency $\omega_{o}$ as follows

$$
E_{c}(t)=j \sqrt{\kappa_{1}} \gamma \sqrt{2 P_{o}} e^{j\left(\omega_{o} t\right)} .
$$

Here, $j$ is the imaginary unit, $\kappa_{1}$ is the power coupling coefficient of the first tunable coupler, and $\gamma$ is a constant relating the field and the average power 


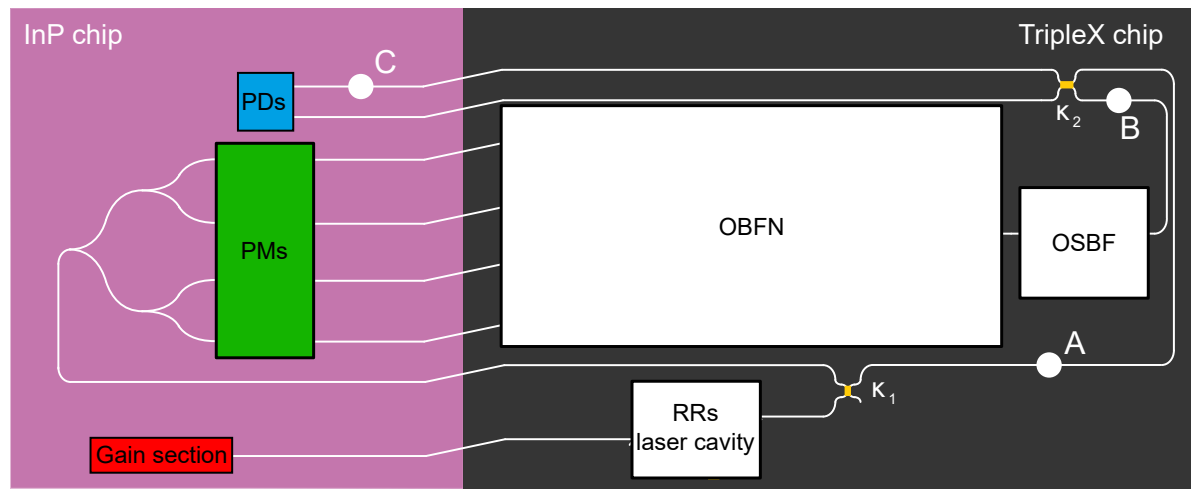

Figure 6.6: Photonic beamformer receiver architecture with optical SSBsuppressed-carrier (SC) modulation and single detection

such that $P_{o}=\left(E_{c}(t) \cdot E_{c}(t)^{*}\right) /\left(2 \gamma^{2}\right)$. For the optical field after the OSBF at point $\mathrm{B}$, for restricting to simple, first-order expressions, assumptions are made. First let us assume that the splitting of the unmodulated carrier is lossless. Let us name $\alpha_{P M}$ the field transmission coefficient related to the phase modulators. Small-index modulation is assumed, such that only the first order side-frequencies have to be considered. The output of the OBFN is a delayed replica of the received signal, assuming again the absence of losses in combining and phase synchronization of the signals coming from the phase modulators. After the filter, characterized by a field transmission coefficient equal to $\alpha_{F}$, only the upper first-order side-frequency is kept, as shown in Figure 6.4. With these assumptions, the optical filed at point B can be written as

$$
E_{o s b f}(t)=\sqrt{\left(1-\kappa_{1}\right)} \alpha_{P M} \alpha_{F} \gamma \sqrt{2 P_{o}} J_{1}\left(\frac{V_{R F} \pi}{V_{\pi, R F}}\right) e^{j\left(\omega_{o} t+\omega_{R F} t+\phi(t)\right)} .
$$

Here, $J_{1}$ is the Bessel function of the first kind, which determines the amplitude of the first order sidebands of the phase-modulated signal. This sideband amplitude depends on the amplitude of the modulating RF signal, expressed as the bandpass signal $V_{i n}(t)=V_{R F} \sin \left(\omega_{R F} t+\phi(t)\right)$, and the $V_{\pi}$ of the modulator. The two optical fields, $E_{c}(t)$ in (6.3) and $E_{\text {osbf }}(t)$ in (6.4), are combined by the lossless tunable coupler characterized with a power coupling coefficient equal to $\kappa_{2}$. Therefore, the optical field at point $\mathrm{C}$ can be written as

$$
E_{p d}(t)=\sqrt{\left(1-\kappa_{2}\right)} E_{o s b f}(t)+j \sqrt{\kappa_{2}} E_{c}(t) .
$$

The photocurrent $I_{p d}(t)$ at the photodiode PD is calculated as

$$
I_{p d}(t)=R_{p d} P_{p d}(t)=R_{p d} \frac{E_{p d}(t) \cdot E_{p d}(t)^{*}}{2 \gamma^{2}} .
$$

Here, $R_{p d}$ is the photodetector responsivity, which measures the electrical output current per optical input power. Inserting (6.5) into (6.6) and assuming 


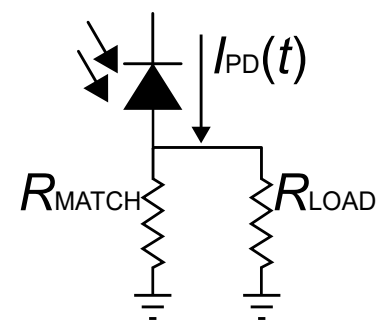

Figure 6.7: Schematic circuit for having single detection

the power coupling coefficients as $\kappa_{1}=\kappa_{2}=0.5$, the photocurrent $I_{p d}$ is given by

$$
\begin{aligned}
I_{p d}(t) & =\frac{1}{4} R_{p d} P_{o}+\frac{1}{4} R_{p d} \alpha_{P M}^{2} \alpha_{F}^{2} P_{o} J_{1}^{2}\left(\frac{V_{R F} \pi}{V_{\pi, R F}}\right) \\
& -\frac{1}{2} R_{p d} \alpha_{P M} \alpha_{F} P_{o} J_{1}\left(\frac{V_{R F} \pi}{V_{\pi, R F}}\right) \cos \left(\omega_{R F} t+\phi(t)\right) .
\end{aligned}
$$

From the photocurrent, referring to Figure 6.7 where the electric circuit for single-diode detection (as opposed to balanced detection with two diodes) is shown, the average RF power can be calculated as

$$
P_{R F}=\frac{1}{4}\left\langle I_{p d}(t)^{2}\right\rangle R_{\text {load }} .
$$

Here, $\left\langle I_{p d}(t)^{2}\right\rangle$ is the time average value of the photocurrent, that can be calculated as $\left(I_{\text {pd,peak }}^{2} / 2\right)$, through the load resistance $R_{\text {load }}$. The factor 4 in the denominator is due to the impedance matching circuitry, since the current flowing through the load resistor, $R_{\text {load }}$, is $50 \%$ of the photocurrent. Inserting eq. (6.7) into eq. (6.15), the average RF power at the fundamental frequency can be calculated and it is expressed as

$$
P_{R F}=\frac{1}{4} \frac{1}{8} R_{p d}^{2} \alpha_{P M}^{2} \alpha_{F}^{2} P_{o}^{2} J_{1}^{2}\left(\frac{V_{R F} \pi}{V_{\pi, R F}}\right) R_{\text {load }}
$$

By applying the small signal approximation for the Bessel function, $J_{n}(\beta) \approx$ $\beta^{n} /\left(2^{n} n\right.$ !), which holds for $\beta<<1$, and is valid if $V_{R F}<<V_{\pi, R F} / \pi$, the resulting small-signal output power at the fundamental is

$$
P_{R F, s s}=\frac{1}{4} \frac{1}{32} R_{p d}^{2} \alpha_{P M}^{2} \alpha_{F}^{2} P_{o}^{2} \frac{V_{R F}^{2} \pi^{2}}{V_{\pi, R F}^{2}} R_{l o a d} .
$$

At this point, the small-signal RF gain can be obtained from (6.10) by inserting $V_{R F}^{2}=2 P_{R F, i n} R_{i n}$, where $P_{R F, \text { in }}$ is the average $R F$ input power and $R_{i n}$ is the input resistance:

$$
g=\frac{P_{R F, s s}}{P_{R F, \text { in }}}=\frac{1}{4} \frac{1}{16} R_{p d}^{2} \alpha_{P M}^{2} \alpha_{F}^{2} P_{o}^{2} \frac{\pi^{2}}{V_{\pi, R F}^{2}} R_{\text {in }} R_{\text {load }}
$$




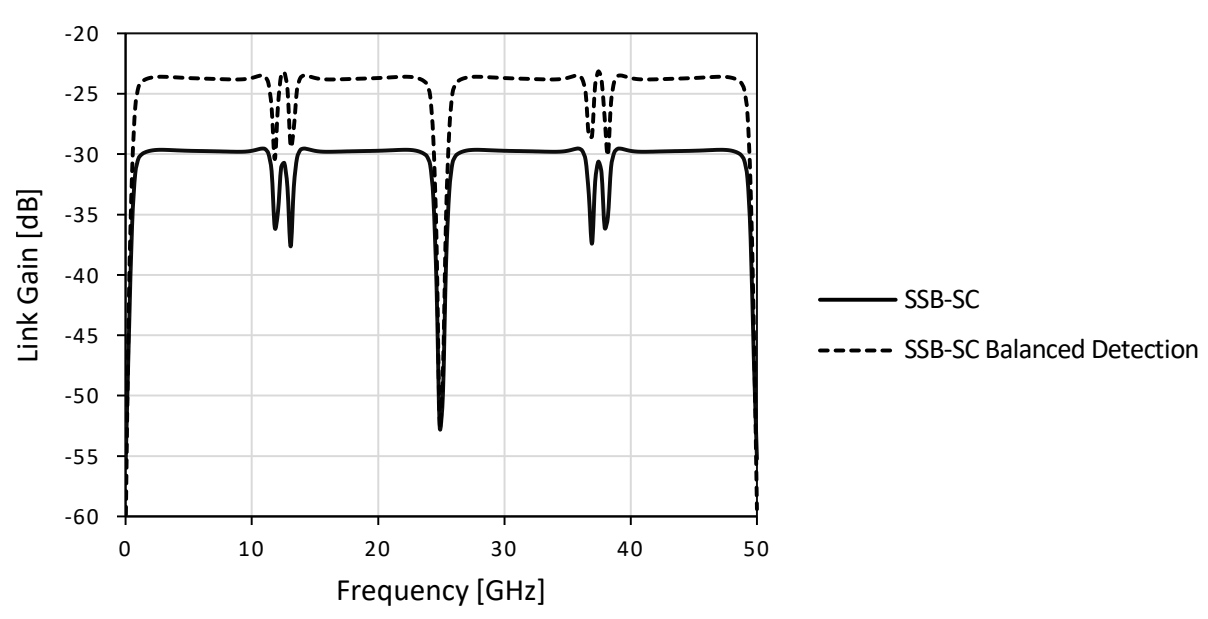

Figure 6.8: Comparison of calculated link gain in the case of SSB-SC with single detection (solid line) and SSB-SC with on-chip balanced detection. The following parameters have been used for running the simulation: $R_{p d}=$ $0.9 \mathrm{~A} / \mathrm{W}, P_{o}=10 \mathrm{dBm}, \alpha_{P M}=0,707(-3 \mathrm{~dB}), \alpha_{F}=1, R_{\text {in }}=R_{\text {load }}=$ $50 \Omega, V_{\pi}=3.8 \mathrm{~V}$.

As an illustration of the typically involved numbers, we show in Figure 6.8 the link gain calculated for an MWP system based on SSB-SC and carrier reinsertion with single detection, based on the schematic of Figure 6.6. The optical carrier is kept at a fixed frequency in the stopband of the filter, as was described in Figure 6.4, while the RF signal applied to the modulators is swept from $10 \mathrm{MHz}$ to $50 \mathrm{GHz}$. A matched load circuitry has been assumed for the modulator side. The following parameters have been used to perform the calculation, in the form of a simulaton using VPIphotonics software [45]: the $\mathrm{RF}$ input power available at the modulator, $P_{R F, \text { in }}$ is set to be $0 \mathrm{dBm}$, $R_{p d}=0.9 \mathrm{~A} / \mathrm{W}, P_{o}=10 \mathrm{dBm}, \alpha_{P M}=0,707(-3 \mathrm{~dB}), \alpha_{F}=1, R_{\text {in }}=R_{\text {load }}=$ $50 \Omega, V_{\pi, R F}=3.8 \mathrm{~V}$ and the FSR of the OSBF is $25 \mathrm{GHz}$. The solid line in the figure corresponds to single detection and its value is around $-29,6 \mathrm{~dB}$, which is the same value given by eq. (6.11). As a comparison, also the case of balanced detection is shown, as will be hereafter described. Using the SSB-SC modulation approach will lead to have a single beat between the sideband and the optical carrier and therefore a $6 \mathrm{~dB}$ average $\mathrm{RF}$ power decrease with respect to intensity modulation, where both first-order sidebands are kept. However, as explained above, this comes at the expense of an increased overall system complexity. 


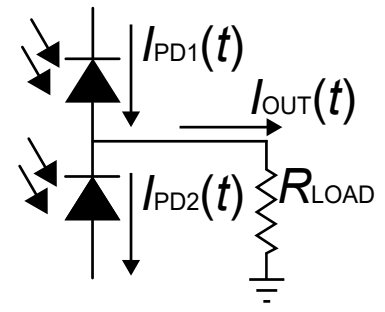

(a)

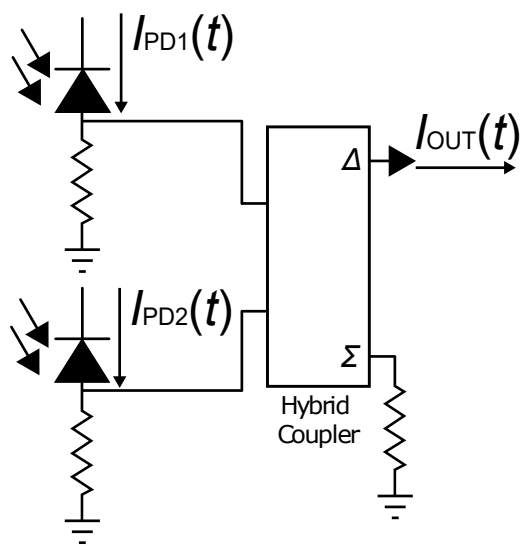

(b)

Figure 6.9: Schematic circuit for a) on-chip balanced detection, b) off-chip balanced detection in electrical domain using $180^{\circ}$ hybrid coupler

\subsubsection{Balanced detection}

The detection can be further improved in the receiver system presented in this chapter, by replacing the single-ended approach (Figure 6.7) with balanced detection. For this type of detection a second photodiode is used in the open output at the right-hand side of Figure 6.6. The advantage is that the amount of received information doubles with regard to single detection. This is achieved by subtracting the two photocurrents as shown in Figure 6.9. The figure shows two possible realizations: the on-chip balanced detection in Figure 6.9 (a) and the off-chip balanced detection in Figure 6.9 (b). In the off-chip option, the currents from the photodiodes are fed into an external RF device, while the on-chip balanced detection makes use of connecting the photodiodes in series, without any external device. In both cases the output current depends on the difference between the optical power incident to the first photodiode and the optical power incident to the second photodiode, which can be expressed as

$$
I_{o u t}(t)=R_{p d}\left(P_{P D 1}(t)-P_{P D 2}(t)\right)=\frac{R_{p d}}{2 \gamma^{2}}\left(\left|E_{p d 1}(t)\right|^{2}-\left|E_{p d 2}(t)\right|^{2}\right) .
$$

Here, it has been assumed for simplicity that the two photodiodes have the same responsivity $R_{p d}$.

$E_{p d 1}$ can be substituted by $(6.5)$, while $E_{p d 2}$ is expressed as follows

$$
E_{p d 2}(t)=\sqrt{\left(1-\kappa_{2}\right)} E_{c}(t)+j \sqrt{\kappa_{2}} E_{o s b f}(t)
$$


Inserting (6.5) and (6.13) into (6.12), the output current is obtained as

$$
I_{\text {out }}(t)=R_{p d} P_{0} \alpha_{P M} \alpha_{F} J_{1}\left(\frac{V_{R F} \pi}{V_{\pi, R F}}\right) \cos \left(\omega_{R F} t+\phi(t)\right) .
$$

The effect of the subtraction of photocurrents in the balanced detector is that the desired RF terms are added up, unlike for instance the DC terms or common mode fluctuations of power in both arms, which are subtracted from each other, improving the dynamic range. Moreover, only the desired RF term at $\omega_{R F}$ is left, doubled with respect to a single-ended detection. Therefore, balanced detection is preferred over single-ended detection.

The difference between the two schematics becomes more clear when the average $\mathrm{RF}$ power is calculated according to (6.15). In the presented system, we have not yet implemented on-chip balanced detection. Instead both photocurrents can be first guided separately off the chip and turned into two voltages before subtraction in a $180^{\circ}$ hybrid coupler. The latter is characterized by a transfer function $H_{h c}=1 / \sqrt{2}$, which together with the matched impedance circuitry will lead to an RF power that can be calculated as

$$
P_{R F, b a l}=\frac{1}{2} \frac{1}{4}\left\langle I_{p d}(t)^{2}\right\rangle R_{\text {load }}
$$

The average RF power for on-chip balanced detection, assuming a small-signal approximation as done in section 6.5 , is given by

$$
P_{R F, b a l, s s}=\frac{1}{4} \frac{1}{8} R_{p d}^{2} \alpha_{P M}^{2} \alpha_{F}^{2} P_{o}^{2} \frac{V_{R F}^{2} \pi^{2}}{V_{\pi, R F}^{2}} R_{l o a d} .
$$

The small-signal RF gain for on-chip balanced detection, and inserting $V_{R F}^{2}=$ $2 P_{R F, \text { in }} R_{i n}$, is given by

$$
g=\frac{P_{R F, b a l, s s}}{P_{R F, \text { in }}}=\frac{1}{4} \frac{1}{4} R_{p d}^{2} \alpha_{P M}^{2} \alpha_{F}^{2} P_{o}^{2} R_{\text {in }} R_{\text {load }} \frac{\pi^{2}}{V_{\pi, R F}^{2}} .
$$

From Figure 6.8, the reader can appreciate the advantage of using balanced on-chip detection over single detection, since the link gain (dotted line) in the first case is $6 \mathrm{~dB}$ higher. Instead, using off-chip balanced detection will reduce this advantage, being the average $\mathrm{RF}$ power $3 \mathrm{~dB}$ lower than the case of on-chip balanced detection.

\subsection{Functional design of the $4 \times 1$ optical beam- forming network receiver}

In this section the functional design of the receiving beamforming system described earlier is presented. The system has been developed in the framework of the Dutch project Promis2Day [46], which was part of the IOP (Innovatiegerichte Onderzoeksprogramma), focusing on Photonic Devices (PD). 


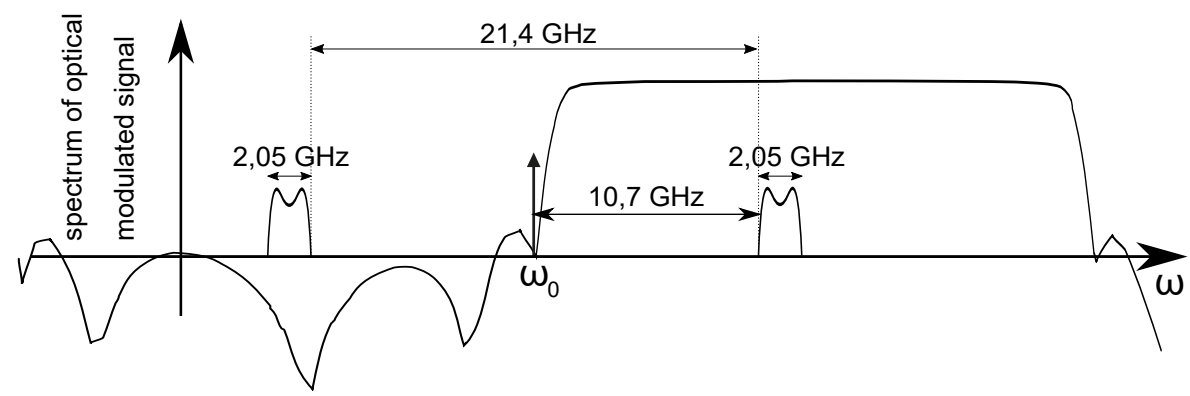

Figure 6.10: Spectrum of optical modulated signal and the desired OSBF for $\mathrm{K}_{\mathrm{u}^{-}}$ band modulating signal in the receiver

\subsubsection{System requirements}

The systems requirements used to develop the prototype described in this chapter are based on the system requirements detailed in the named Promis2Day project. The aim is to realize the first-ever prototype of a full hybrid integrated photonic TTD beamforming system. The prototype is aiming on the control of an $8 \times 8$ antenna tile, as a module of a full-scale antenna, whose total number of antenna tiles (determining the antenna aperture, can be selected depending on the frequency range of interest and the required signal-to-noise ratio. However, to reduce the risk associated with the scientific and technological challenges, we have restricted our system to a smaller structure, namely a $4 \mathrm{x} 1$ receiver.

For providing a better overview, in the following we give a more concrete example into what direction the investigated prototype can be developed. When a digital video signal broadcasted by a satellite (DVB-S) has to be received, the antenna aperture should be around $2300 \mathrm{~cm}^{2}$ and it would require around 25 tiles [24]. Most of the modern systems for mobile satellite services work with $\mathrm{K}_{\mathrm{u}}$ band frequencies. In Europe, the $\mathrm{K}_{\mathrm{u}}$ band is used from 10.7 to $12.75 \mathrm{GHz}$ for broadcasting satellite services such as those carried by the fleet of Astra satellites. The named interval is usually divided in two segments of frequencies, the first from 10.7 to $11.7 \mathrm{GHz}$ and the second from 11.7 to $12.5 \mathrm{GHz}$. Two signal processing approaches are possible.

The first is to convert the $\mathrm{K}_{\mathrm{u}}$ frequencies directly into the optical domain and process them through the OBFN and the OSBF. Figure 6.10 describes graphically this situation. The OBFN should provide a constant group delay response over the range from 10.7 to $12.75 \mathrm{GHz}$, which corresponds to a bandwidth of $2.05 \mathrm{GHz}$. From this requirement on the bandwidth, it is possible to define the number of RRs needed to guarantee the proper delay functionality. Considering the upper frequency limit of $12.75 \mathrm{GHz}$, the maximum interelement distance of the $8 \times 8$ antenna tile for avoiding grating lobes is $1.18 \mathrm{~cm}$, and the desired beam scanning range is $\left[-60^{\circ}, 60^{\circ}\right]$. Therefore, the maximum delay 
Table 6.1: Frequency requirements for the OSBF for the receiver in the case of $K_{u}$ frequency range

\begin{tabular}{||c|c|c|c||}
\hline System & Passband BW & Stopband BW & Transition BW \\
\hline \hline Receiver & $>2.05 \mathrm{GHz}$ & $>12.75 \mathrm{GHz}$ & $<10.7 \mathrm{GHz}$ \\
\hline
\end{tabular}

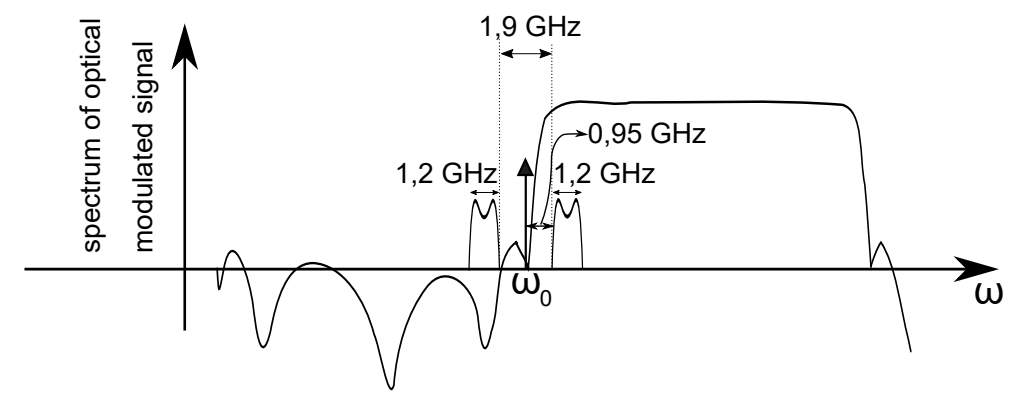

Figure 6.11: Spectrum of optical modulated signal and the desired OSBF for Lband modulating signal in the receiver

that the OBFN has to provide to synchronize the signals within one tile is 238 ps, according to Section 4.4.

The OSBF can be designed upon graphical inspection of Figure 6.10. From here, the values for the main parameters of the filter can be derived, such as the passband bandwidth, the stopband bandwidth and the transition bandwidth, as summarized in Table 6.1. These values are used for determining the FSR of the OSBF.

The second approach requires that the receiver down-converts the signals from $\mathrm{K}_{\mathrm{u}}$ to an intermediate frequency (IF) range, and processes the IF in the optical domain. The IF in a standard European transceiver extends from 950 $\mathrm{MHz}$ to $2.15 \mathrm{GHz}$. To cover this range, two different local oscillators are used with their frequencies at $9.75 \mathrm{GHz}$ and $10.6 \mathrm{GHz}$, down-converting respectively the first frequency block, $(10.70-11.70 \mathrm{GHz})$, and the second frequency block (11.70-12.75 GHz). This second approach is represented in the RF domain in Figure 6.11. In this case, since the signal bandwidth is $1.2 \mathrm{GHz}$, the optical bandwidth requirements to be fullfilled by the OBFN are relaxed with respect to the first approach. Nevertheless, the requirements on the OSBF are more stringent, especially for the transition bandwidth, which has to be small enough since the sidebands are much closer to the optical carrier. The requirements in terms of frequency specifications for the OSBF are summarized in Table 6.2 as well.

The named parameters associated with the two approaches will ultimately determine the number of rings needed in the OBFN and the FSR of the OSBF. However, even though the physical layouts of the according chips will be slightly 
Table 6.2: Frequency requirements for the OSBF for the receiver in the case of Lband intermediate frequency range

\begin{tabular}{||c|c|c|c||}
\hline System & Passband BW & Stopband BW & Transition BW \\
\hline \hline Receiver & $>1.2 \mathrm{GHz}$ & $>2.15 \mathrm{GHz}$ & $<0.95 \mathrm{GHz}$ \\
\hline
\end{tabular}

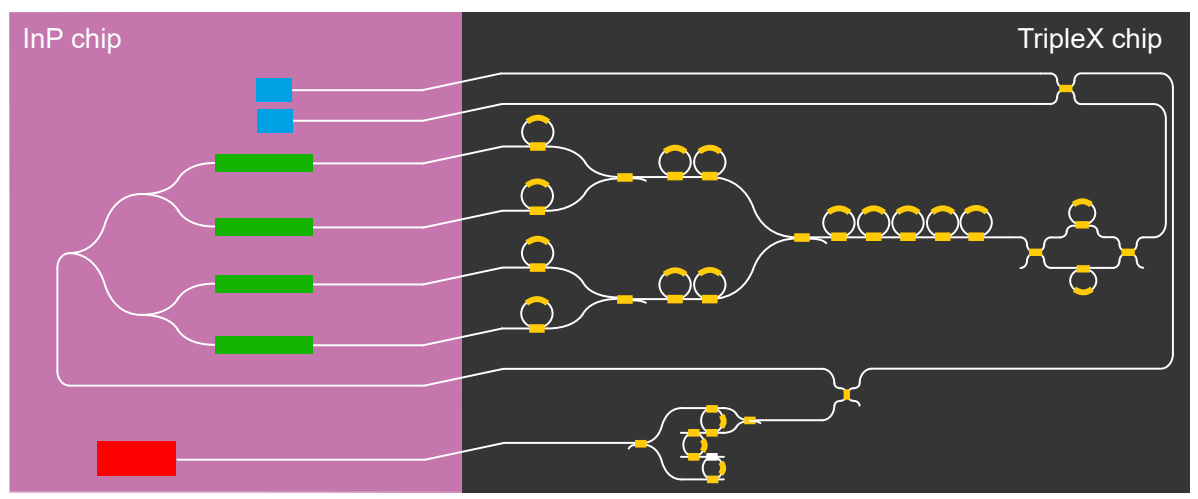

Figure 6.12: Functional design of the hybrid integrated OBFN receiver system

different, the same functional design shown in Figure 6.12 can apply to the receiver for processing the IF band as well as to the receiver for processing the $\mathrm{K}_{\mathrm{u}}$ band. The difference is due to the differential path length in the two arms of the OSBF, since the two OSBF are required to have a different FSR.

The functional design of the hybrid integrated photonic receivers is shown in Figure 6.12, where the building blocks described earlier are properly arranged. The hybrid integrated laser, comprising the gain diode (in red) receiving optical feedback from the external arm of the laser cavity with the three RRs, provides a very stable optical carrier with narrow linewidth. The optical carrier is split by means of a tunable coupler before entering modulation (in green), such that part of the carrier remains available for the re-insertion to perform coherent detection at the photodiode(s) (in blue). The part of the carrier used for modulation is split into four branches in the InP chip. The four phase-modulated signals are then processed by the $\mathrm{OBFN}$ in the $\mathrm{Si}_{3} \mathrm{~N}_{4}$ waveguide (TripleX ${ }^{\mathrm{TM}}$ ) chip. For the OBFN, the delay elements, i.e. the RRs, are required to provide the desired delay across a minimum bandwidth that covers the sideband that will be retained after the OSBF. Note that the OSBF would be needed after each phase modulator before the OBFN. However, in this case, there would be as many filters as AEs, which means complexity in the mask layout of the chip. A solution to this, after noticing that the OBFN and OSBF are linear devices, is that the order of components can be reversed and as a result only one common OSBF will be needed.

Overall, the delay ripple should be kept in the order of tens of picoseconds, 


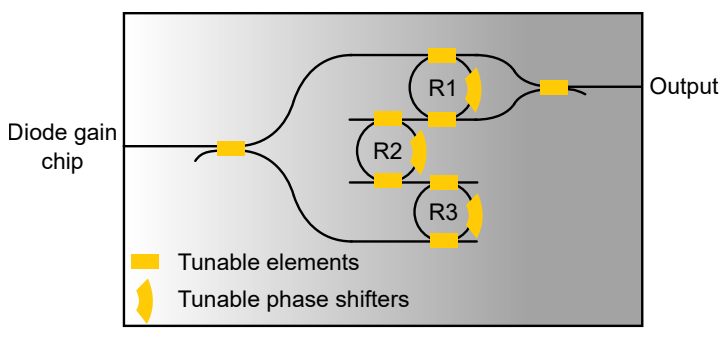

Figure 6.13: Functional design of the external cavity of the hybrid integrated laser

which corresponds to a phase error in the delayed signals lower than $\pi / 16$, such that the effect on the antenna pattern is negligible [47, 48]. The OSBFs should be designed according to specifications in Table 6.1 and in Table 6.2, while the insertion loss has to be kept as low as possible. Single detection can be performed as well as balanced detection (via an off-chip hybrid coupler), since two photo-detectors have been fabricated on the InP chip (depicted in blue).

\subsubsection{Design and measurements of the hybrid integrated laser}

The design of the feedback circuit for the hybrid laser is shown in Figure 6.13. Next to the three RRs use for frequency selection and tuning, the external cavity contains also two tunable couplers, one at the input side and the other at the output side of the cavity. These are used as preparation for later steps of investigation and optimization, such as for coherently adding the output power emerging from different ports, or for monitoring purposes. The lengths of the three rings are chosen as $L_{R 1}=9899 \mu \mathrm{m}, L_{R 2}=9690 \mu \mathrm{m}$, and $L_{R 1}=$ $9500 \mu \mathrm{m}$. Each of the three RRs is fully tunable, meaning that the power coupling coefficients are tunable as well as the phase shifters. Full tunability was implemented for imposing single-frequency oscillation and for exploring, at a later stage, the option of a trade off between narrow bandwidth and higher output power.

Figure 6.14 shows the output spectra of the hybrid integrated laser when operating at a driving current of $192 \mathrm{~mA}$ in two different cases. In Figure 6.14 (a), the RRs-based mirror has not been properly tuned and the emission spectrum of the hybrid integtrated laser is clearly multi-mode. However, with tuning the 11 heating elements present, the emission spectrum can be changed from multi-mode to single-mode, in this example at a wavelength of $1555 \mathrm{~nm}$. The output power is about $4 \mathrm{dBm}$ and the side mode suppression ratio (SMSR) is higher than $50 \mathrm{~dB}$, which satisfies the requirements on high spectral purity [49]. 


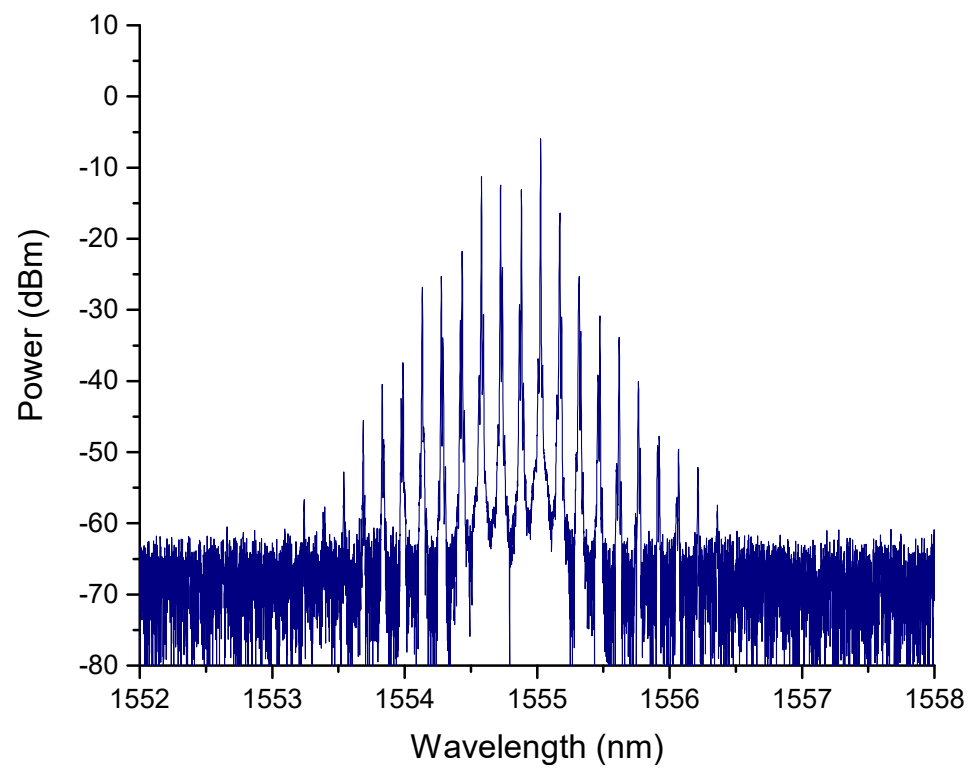

(a)

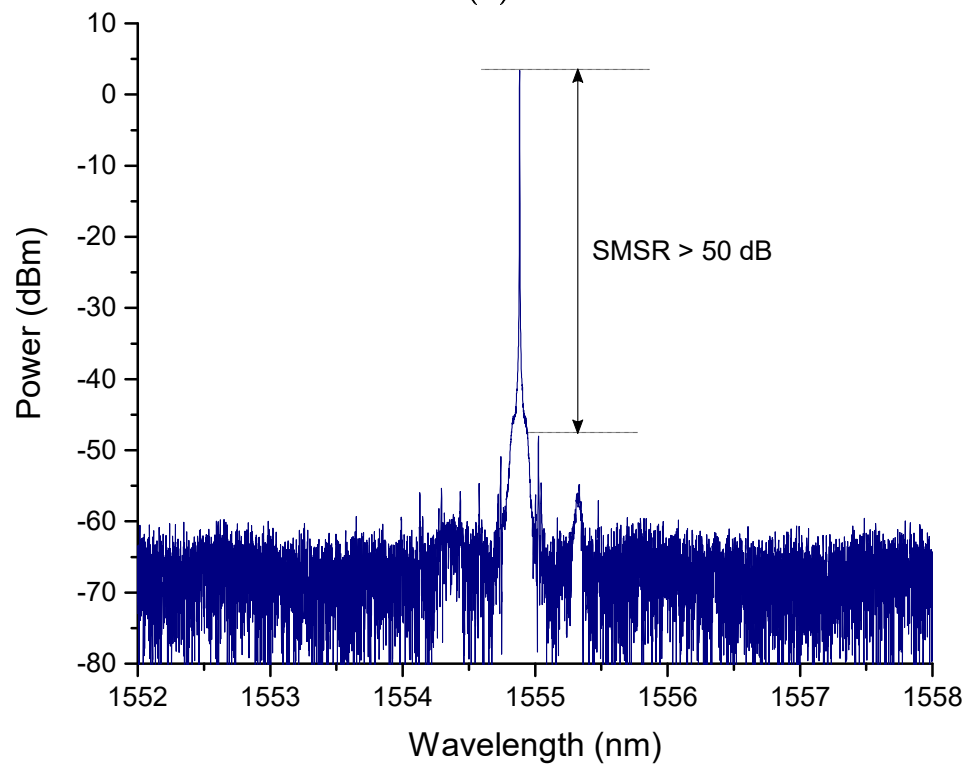

(b)

Figure 6.14: Measured emission spectrum of the hybrid integrated laser as a function of wavelength obtained when a driving current of $192 \mathrm{~mA}$ is applied to the gain diode. In a) the RRs are not properly tuned and the laser oscillates at multiple modes. In b) single-mode oscillation is seen after properly setting the 11 tunable elements. The measured side mode suppression ratio (SMSR) is larger than $50 \mathrm{~dB}$. 
Table 6.3: Calculated optical parameters for the OSBF filter

\begin{tabular}{||c|c||}
\hline Optical parameter & Value \\
\hline \hline$\kappa_{1}$ & 0.5 \\
\hline$\kappa_{2}$ & 0.5 \\
\hline$\kappa_{u r}$ & 0.32 \\
\hline$\kappa_{l r}$ & 0.87 \\
\hline$\phi_{m z i}$ & $0^{\circ}$ \\
\hline$\phi_{u r}$ & $180^{\circ}$ \\
\hline$\phi_{l r}$ & $180^{\circ}$ \\
\hline
\end{tabular}

\subsubsection{Design and measurement of optical sideband filter}

The structure of the filter used to transfer phase modulation into SSB-SC modulation has been already presented in section 3.3.0.5. For completeness, let us recall that it consists of an AMZI loaded with two rings, one at each branch, as shown in the inset of Figure 6.15. The roundtrip of the RRs is twice the differential path between the two branches. Also, the other optical parameters such as the power coupling coefficients for the directional couplers of the AMZI, the power coupling coefficients to the RRs as well as the phase of the RRs and the phase of the AMZI are tunable, i.e., it is possible to change the filter resonance frequencies after fabrication. This gives to the overall system a great flexibility. To give an example, if the optical carrier wavelength would show a drift, e.g., due to changing temperature, the filter could be shifted accordingly, without changing its other main properties such FSR, the stopband rejection, the passband bandwidth and the transition bandwidth. On top of this main feature for system flexibility, having a filter that is adjustable also with regard to its other parameters would have the advantage that the technological challenge of fabricating perfect directional couplers is much removed. Essentially, the challenge of fabricating the device right to its design value without deviations is replaced by proper tuning the device after fabrication. Thereby, deviations from ideal values, which cannot be avoided in any device fabrication, can be compensated.

In order to approach the described goals as good as possible, we started the design considerations with the definition of the FSR. The system requirements needed to select the FSR for operation in the $\mathrm{K}_{\mathrm{u}^{-}}$and $\mathrm{L}-$ bands have been described above and are summarized in Table 6.1 and Table 6.2. The theoretical description of the filter transmission spectrum was carried out by making use of the $z$-transform via specifying the order of the filter, the bandwidth, the allowed ripple in the passband and stopband, the stopband rejection, and the transition bandwidth. In our case, these requirements are the following: a flattened passband bandwidth corresponding to $46 \%$ of the FSR (defined at $-0.01 \mathrm{~dB}$ ), and at least $25 \mathrm{~dB}$ of suppression in the stopband with equiripples, resembling a type-2 Chebyshev filter. A $z$-transform which satisfies the aforementioned 


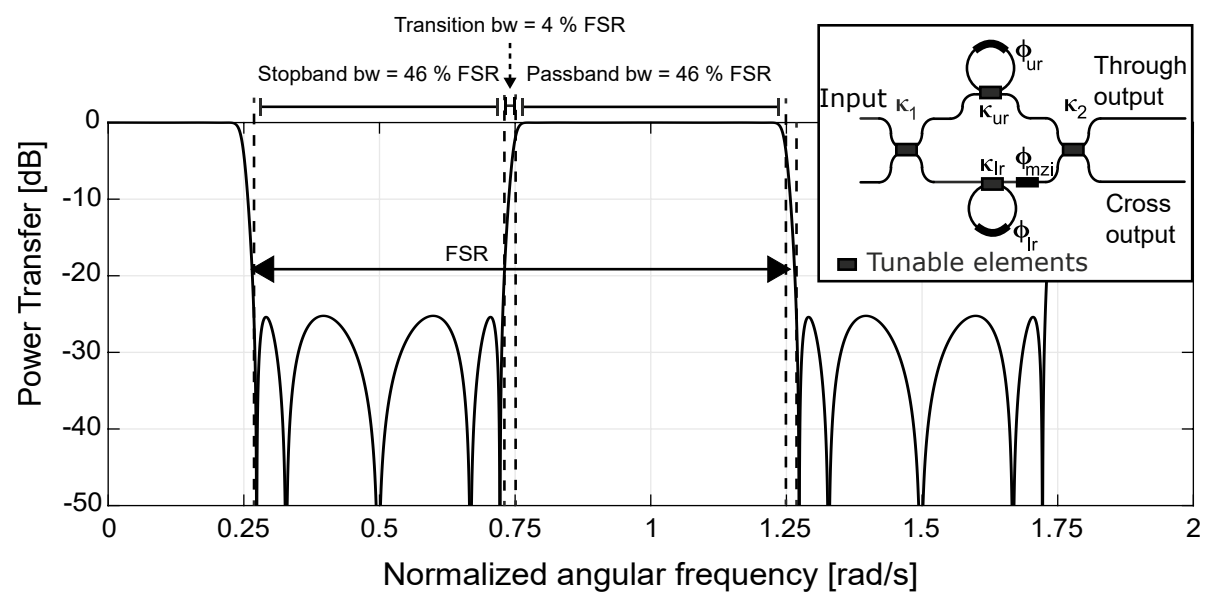

(a)

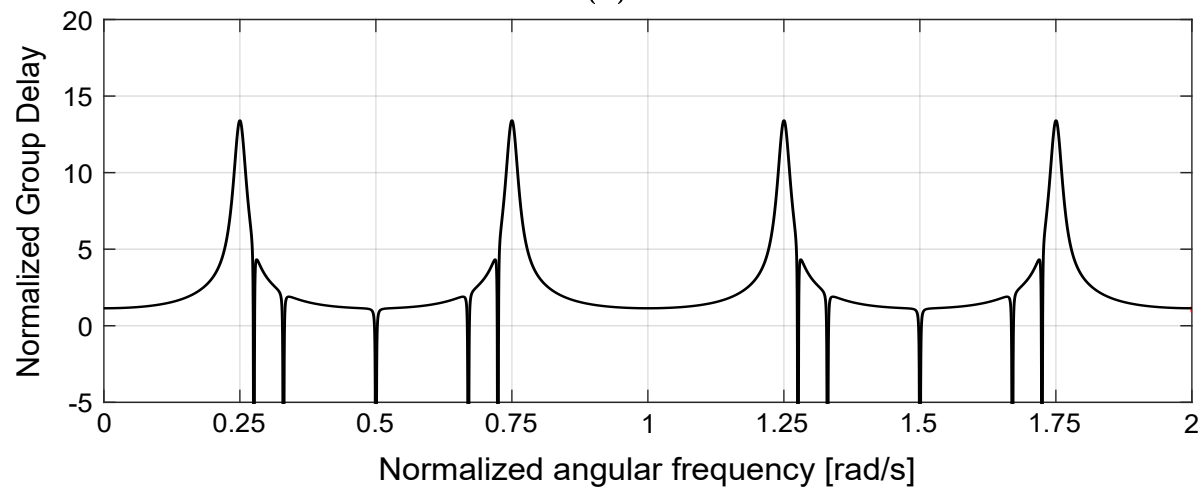

(b)

Figure 6.15: (a) Calculated power transfer function of the through port of the OSBF as designed. In the inset, the waveguide circuit structure of the filter is shown; (b) Group delay response of the through port normalized to the number of roundtrips 


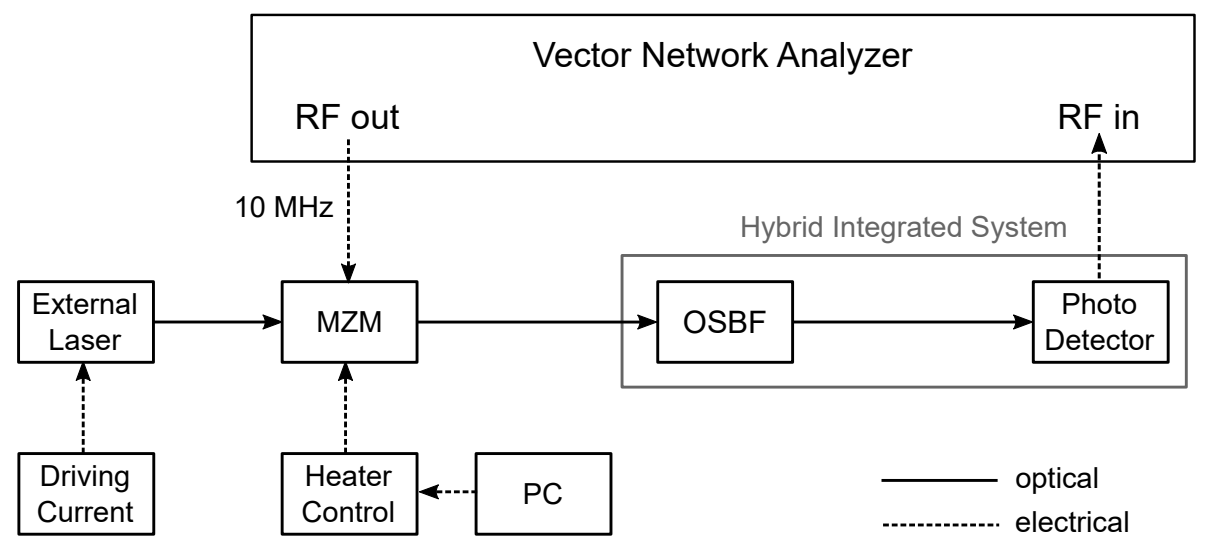

Figure 6.16: Measurement setup for OSBF characterization

requirements can be written as:

$$
\begin{aligned}
& H_{\text {through }}(z)= \\
& \frac{-0.1803+0.4123 z^{-1}-0.6487 z^{-2}+0.6487 z^{-3}-0.4123 z^{-4}+0.1803 z^{-5}}{1+1.852 z^{-2}+0.2973 z^{-4}}
\end{aligned}
$$

From the $z$-transform coefficients and a synthesis algorithm, as described in [37], the optical parameters of the AMZI loaded with the two RRs, can be calculated and are given in Table 6.3. In Figure 6.15(a) and Figure 6.15(b), the calculated power transfer spectrum and normalized group delay of the filter are depicted, respectively, using the optical parameters of Table 6.3. The spectra are plotted over an interval of two FSRs. A full tunability of the device is obtained by using thermo-optical tuning elements. In particular, such OSBF comprises two tunable couplers, two RRs, and three phase shifters, which means a total number of seven tuning elements. The chosen FSR for the $\mathrm{K}_{\mathrm{u}}$-band and L-band are $50 \mathrm{Ghz}$ and $25 \mathrm{GHz}$, respectively.

\subsubsection{Measurement setup for the OSBF characterization}

The OSBF power transfer response was measured using a wavelength-sweeping approach, the setup of which is graphically presented in Figure 6.16. The hybrid integrated system depicted in Figure 6.12 has been designed such that some parts of it can be accessed externally by means of test ports. The OSBF can be separately accessed using an external CW laser modulated by an external MZM modulator biased at quadrature point. This allows a fast preliminary check of the filter functionality, without having to rely on properly tuning the feedback circuit of the hybrid integrated laser. The wavelength-sweeping ap- 
proach is implemented as follows. First, the $\mathrm{CW}$ laser is modulated by an external modulator with a fixed-frequency, which in our case had an average power of $10 \mathrm{dBm}$ and a frequency of $10 \mathrm{MHz}$, generated by the VNA. Secondly, the wavelength of the $\mathrm{CW}$ laser is swept by ramping its driving current. Depending on the amplitude of the ramp, the wavelength of the laser will span a certain frequency range and the photodetector will produce a current which is proportional to the average output power at the detecting photodiode generated by the spectral triplet, made of the carrier, the upper side-frequency and lower side-frequency, assuming a small modulation index. The output of the photodiode is fed back into the VNA for performing a measurement of the link gain. In this way it is possible to record the RF amplitude and phase response of the OSBF.

In our measurement setup, the optical carrier is generated by a Gooch \& Housego high-power 14-pin distributed feedback laser (AA1406-195600-100PM900-FCA-50) with a maximum output of $100 \mathrm{~mW}$. The specified linewidth of the laser, $\Delta \nu$, is $1 \mathrm{MHz}$, which corresponds to a coherence time of $\tau_{c}=$ $1 /(\pi \Delta \nu)=0.32 \cdot 10^{(-6)} s$. The intensity of the optical carrier is modulated by means of a Covega Mach-Zehnder modulator, which is a lithium-niobate modulator with a $3 \mathrm{~dB}$ bandwidth of $10 \mathrm{GHz}$. The RF signal is generated by a Rohde \& Schwarz ZNB VNA with an operational bandwidth of $40 \mathrm{GHZ}$. All the optical fibers used in the setup are polarization mantaining since the waveguides of the chip are polarization-dependent. The OSBF is controlled by thermal tuning. Each heater of the OSBF can be addressed by a USB-controlled power supplies and a customized software via a laptop. The optical throughput is measured with the on-chip integrated photodetector from SMART Photonics, which has a bandwidth of about $10 \mathrm{GHz}$.

\subsubsection{Measurement results for the OSBF characterization}

Using the measurement setup and methods described in section 6.6.3.1, the frequency response of the OSBF filter has been characterized. In Figure 6.17(a) and Figure 6.17(b), the results of a measurement session on the OSBF designed for the L-band are shown in terms of power transfer and normalized group delay. The filter was chosen to provide an FSR of $25 \mathrm{GHz}$. The reason of the choice of the L-band system as example for the characterization was mainly limited by the speed of the modulators available at the time of the measurements. The measurements proved that the filter functions as expected, in good agreement with the simulation, showing a passband of $11.5 \mathrm{GHz}$ bandwidth, a steep transition bandwidth and a suppression of about $20 \mathrm{~dB}$. A remaining small discrepancy between the simulation and the measurement in the stopband can be addressed to deviations from optimum experimental parameter settings because tuning was carried out manually so far. We believe that further optimization can be done so that all stopband ripples are at the same height. However, a more appropriate approach for setting the optical parame- 


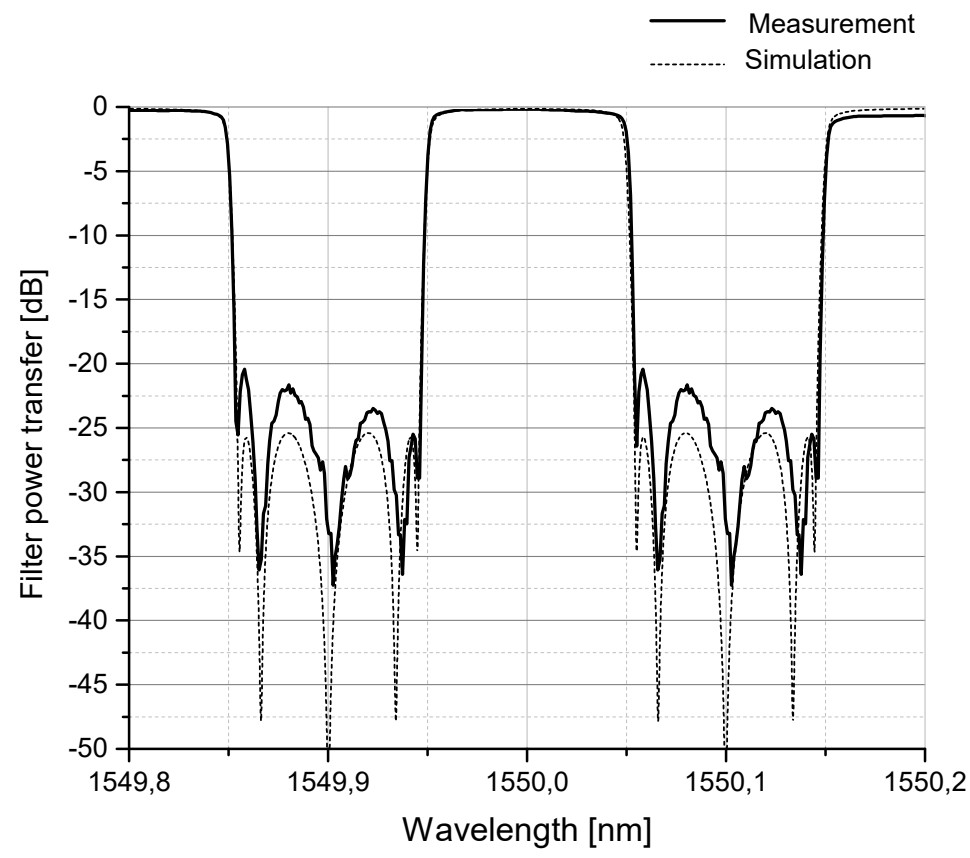

(a)

Measurement Simulation

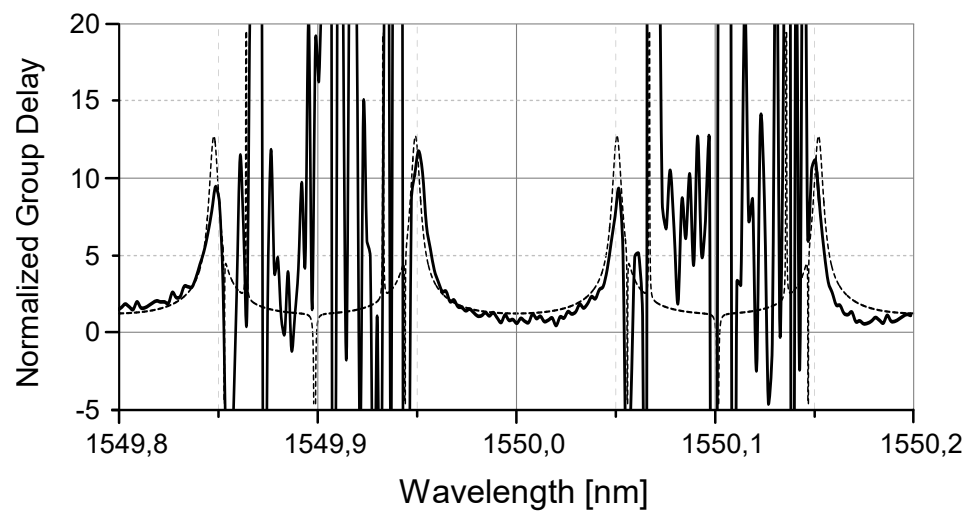

(b)

Figure 6.17: (a) Measured and calculated OSBF power transfer response spectrum of the OSBF and (b) the according normalized group delay. Calculation have been obtained by simulate the responses considering a waveguide propagation loss of $0.2 \mathrm{~dB} / \mathrm{cm}$ 


\section{Stage $1 \quad$ Stage 2}

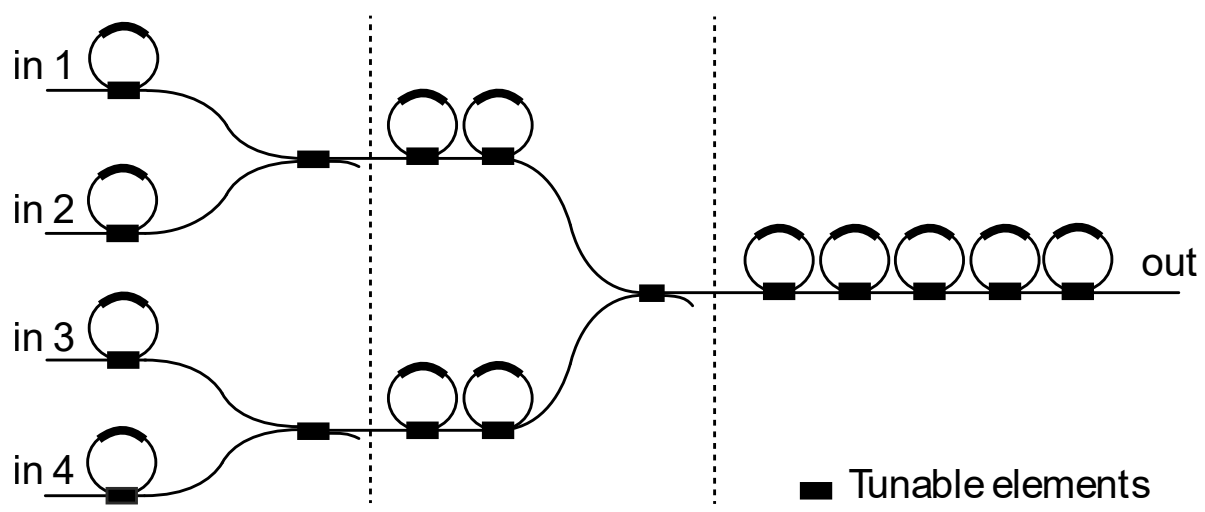

Figure 6.18: Structure of the receiving OBFN for the Promis2Day beamforming

ters of the OSBF would be making use of neural networks, as recently shown in $[50]$.

\subsubsection{Design and measurements of the optical beamform- ing network}

The OBFN design of the receiver is described in this section and comprises of a symmetrical binary-tree structure, as shown in Figure 6.18. Here, the OBFN has four inputs to be superimposed with two binary-combining stages. All the couplers are thermally tunable via heater currents, which allows the OBFN to be flexible and reconfigurable. These properties are important for the compensation of unavoidable fabrication errors and to perform its function, to set the direction of reception and to improve the directivity of the received radiation pattern. As was explained in section 6.4, a single $R R$ is required for each branch of the binary-combining sections in the first stage and two cascaded rings for each branch of the binary-combining section of the second stage. The first two stages are necessary to synchronize the signals within one tile, while the five cascaded RRs on the common branch after the last combiner, as shown in Figure 6.18, are used to synchronize the signals from different tiles. Since the delay between different tiles is bigger than within a single tile, more RRs are required to fulfill the delay and bandwidth requirements.

Overall, the receiving OBFN consists of 13 RRs and 3 optical combiners. In order to have the OBFN programmable and re-adjustable, in total 29 tuning elements are to be controlled, for setting power coupling to the rings, to adjust the resonance frequencies of the rings, and to regulate the power coupling of the optical combiners.

In order to keep the occupied area as small as possible, it is desired to have the 


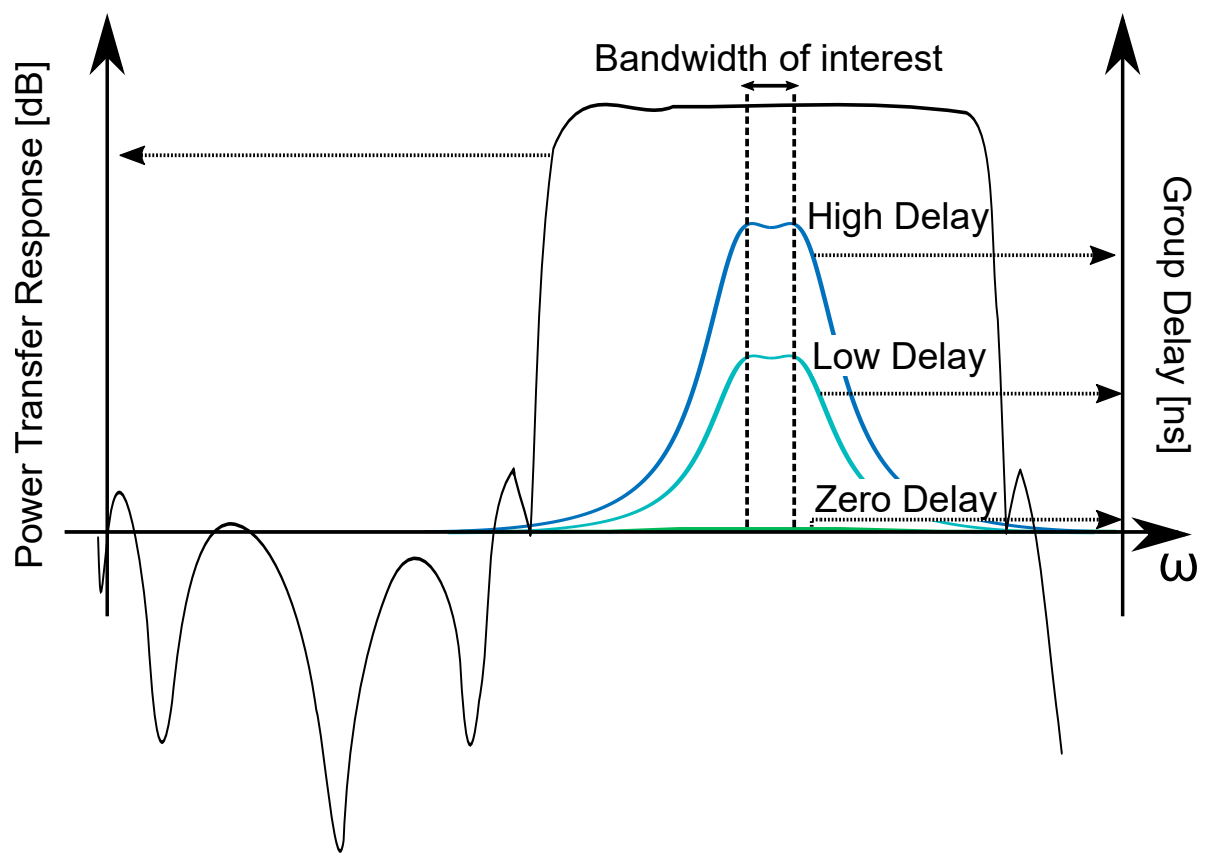

Figure 6.19: Example of how the filter frequency response and group delays produced by the RRs have to be placed with respect to each other

shortest ring achievable, which will in turn have the widest FSR. The RRs were selected to have an FSR of $25 \mathrm{GHz}$, corresponding to a circumference of about $7 \mathrm{~mm}$, which was the smallest value possible at the time of fabrication, where the radiation loss caused by waveguide bending is still small in comparison with the coupling parameters to be selected for operation.

In Figure 6.19, the relative alignment of the filter frequency response with the RRs group delay response versus angular frequency is shown. In order to achieve such alignement, in the experiments the parameter setting was done in the following sequence. First the OSBF was optimized to the desired type2 Chebyshev filter shape. Successively, the RRs are properly set such that the group delay over the bandwidth of interest is constant, besides a residual ripple (smaller than $\pi / 16$ from [24]), and is within the OSBF filter passband. In the case of the L-band receiver, the required group delay bandwidth is 1.2 GHz, as mentioned in section 6.6.1. In order to investigate wether the expected functionality is provided by the OBFN, one of the four possible inputto-output paths was selected as an example, which is the path from input 1 to the output in Figure 6.18. For the measurements, we have optimized the couplers (that combine signals from the other paths), in order to have maximum power in the selected path. The RRs in the path have been tuned to three different configurations. The first configuration is given by tuning all the 


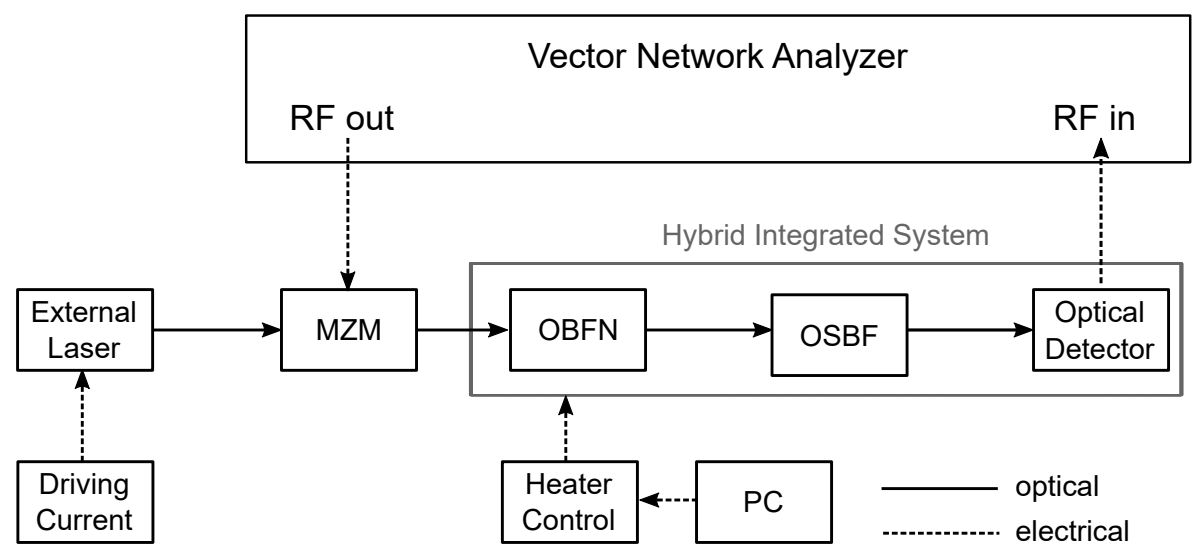

Figure 6.20: Measurement setup for OBFN characterization

tunable couplers of the rings to zero, meaning that the light does not couple to any ring. This setting with zero delay contribution from the rings serves as a reference. For the second and third configuration, two rings have been tuned to have a low and a high group delay within the desired bandwidth. These configurations are labelled as Zero Delay, Low Delay and High Delay in Figure 6.19, respectively.

\subsubsection{Measurement setup for the OBFN characterization}

Figure 6.20 shows the measurement setup that has been used, comprising an external CW laser followed by an external MZM, biased at its quadrature point. For recording a power transfer and delay spectrum, the wavelength of the optical carrier is kept at a fixed value, while the RF frequency of the modulating signal was swept (frequency-sweeping method). However, the relative positioning of the optical carrier with regard to the center frequency of the filter remains very important, since it determines the kind of modulation to be observed, as described in section 6.3 .

To illustrate this to more detail, Figure 6.21 shows the RF power transfer of the filter measured for various different positions of the optical carrier with respect to the filter. For example, in Figure 6.21(a) the wavelength of the optical carrier is set to exactly the middle of the passband of the filter, as shown in the inset. The resulting RF power transfer response then follows the behavioral result already obtained through the wavelength-sweeping, which is a confirmation of the frequency response of the filter. In the second carrier setting, where the wavelength is chosen to lie off the center frequency of the filter response, as shown in Figure 6.21(b), the two sidebands are both within the passband for a certain range of modulation frequencies. For higher modulation 

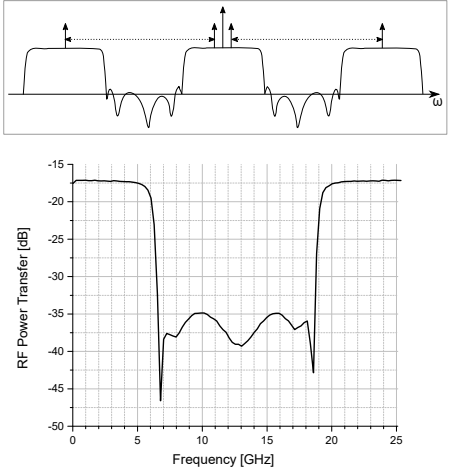

(a)
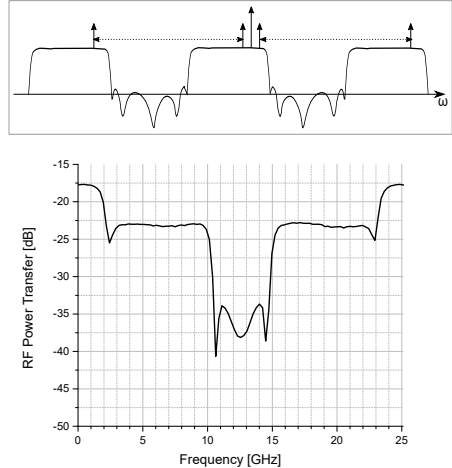

(b)
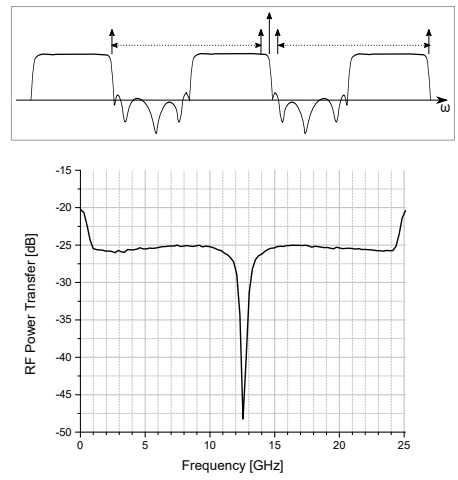

(c)

Figure 6.21: Measured RF power transfer response of the filter using the frequencysweeping method when the optical carrier is a) positioned in the center of the passband of the filter, b) aligned almost at the $3 \mathrm{~dB}$ bandwidth of the filter, and c) aligned at the $3 \mathrm{~dB}$ bandwidth of the filter. In the insets the spectra of the modulated carrier and the filter are depicted, highlighting the position with respect to each other. The dashed arrows indicate tuning of the sidebands by sweeping the RF modulation frequency from a lower to higher value. 


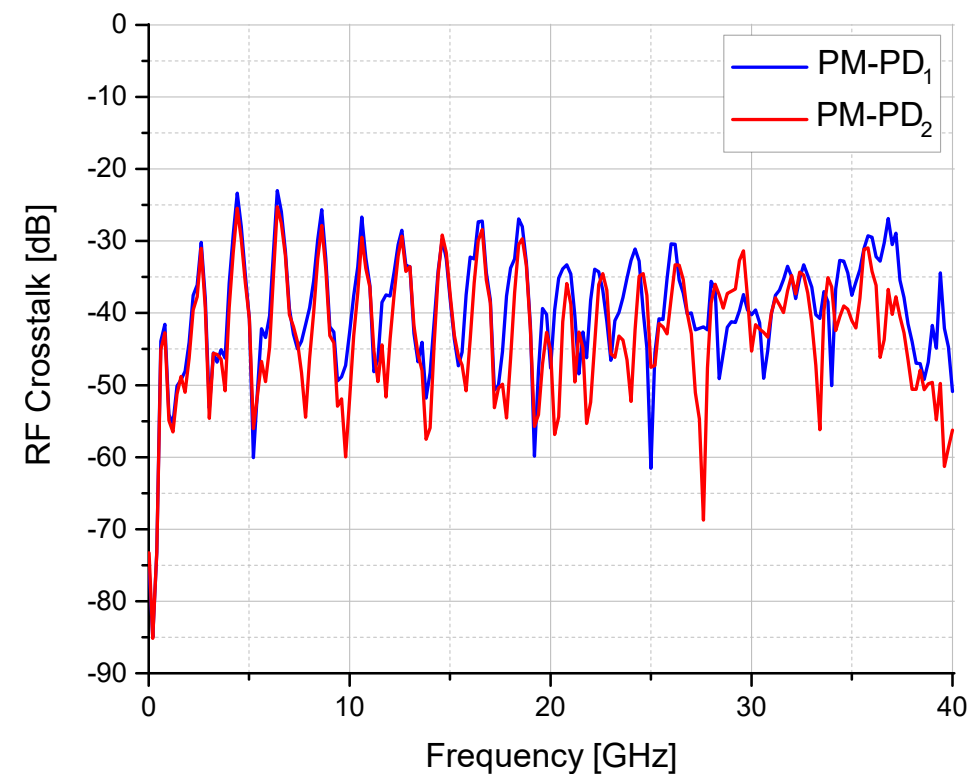

Figure 6.22: RF crosstalk obtained measuring the receiving IMWP system, where an RF sweeping signal is imposed on the closest PM to the photodiode and the detected signals from the 2 photodiodes have led into the VNA

frequencies, one sideband will remain in the stopband while the other will remain in the passband. This situation can be identified as a drop in the power transfer response by $6 \mathrm{~dB}$, because only one sideband is contributing to the beat at the photodiode. For even higher modulation frequencies, at some point both the sidebands will be in the stopband. A very important positioning of the optical carrier is the third one, shown in Figure 6.21(c), since it is used to achieve single sideband full carrier modulation, as there is always only one sideband in the passband while the other is in the stopband and viceversa, as clear looking at the inset. Here, the optical carrier is exactly at the $3 \mathrm{~dB}$ point of the bandwidth of the filter. For obtaining the desired situation of SSB-SC, the carrier was positioned exactly at the first zero of the stopband, to be fully suppressed.

Before presenting experimental results, it is important to mention why an external modulator was used instead of the integrated phase modulators. The reason is that we observed severe RF crosstalk, as shown in Figure 6.22, when two active components are integrated on the same InP chip. The RF signals from the photodiodes shown are due to the modulators that are located near the photodetectors. We measured that the modulator-photodiode crosstalk 


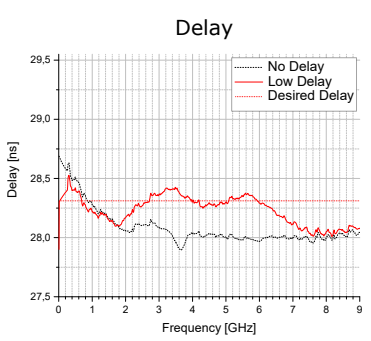

(a)

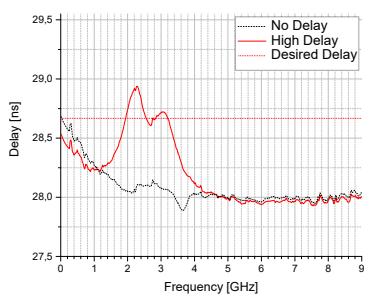

(d)

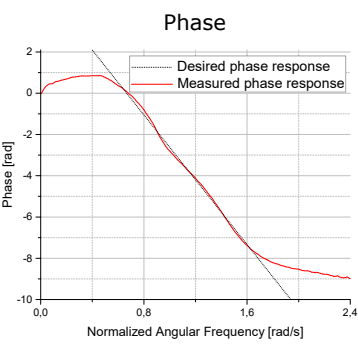

(b)

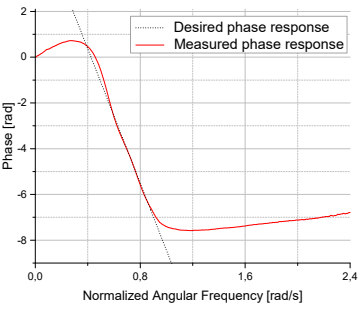

(e)

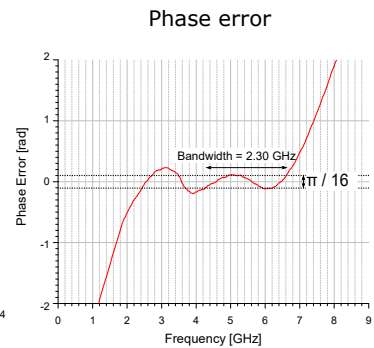

(c)

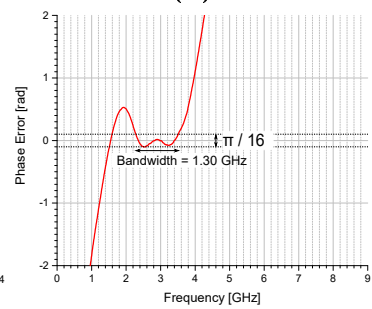

(f)

Figure 6.23: Measured group delays for two different settings of the RRs for achieving (a) low delay compared to the desired delay with the respective (b) RF phase response and (c) phase error when compared to the phase response of the desired delay; for achieving (d) high delay compared to the desired delay with the respective (e) RF phase response and (f) phase error when compared to the phase response of the desired delay

is as high or even higher than the estimated link gain (the latter could not be measured, but the estimated value was about $20 \mathrm{~dB}$ lower than the $\mathrm{RF}$ crosstalk). In the fabrication runs on which the presented results are based, a possible dominance of RF crosstalk was not taken into account. Certainly the next circuit designs as well as packaging considerations will have to put much emphasis also on the $\mathrm{RF}$ radiation, in addition to the optical radiation. However, the experiment has been performed using an external MZM modulator and integrated photodiodes on the InP.

\subsubsection{Experimental characterization of the OBFN}

After the positioning of the optical carrier to provide SSB-SC, the RF modulating signal has been swept from $10 \mathrm{MHz}$ to $9 \mathrm{GHz}$ and the group delay response has been measured. The measurements results in Figure 6.23(a) and Figure 6.23(d) show the cases of setting the RRs to the low delay and high delay, respectively, which correspond to about $0,3 \mathrm{~ns}$ and $0,5 \mathrm{~ns}$ when compared to the reference path, named no delay in the figure. The respective phases are depicted in Figure 6.23 (b) and (e). They are compared with the phase response the desired delay, which is a straight line having slope equal to the 
value of the desired delay. This straight line was used to calculate thephase error compared to the measured phase response given by the combination of RRs. Let us recall that the objective is to have a phase ripple smaller than $\pi / 16$ (from [24]) within the bandwidth of interest, which in our specific case is $1.2 \mathrm{GHz}$.

The calculated phase error for the two delays is plotted in Figure 6.23(c) and (f). Here, we can see that the low delay shows a phase ripple smaller than $\pi / 16$ for a bandwidth of $2.3 \mathrm{GHz}$, which is almost twice as wide than what required. For the high delay, however, the bandwidth of small ripple is somewhat narrower, about $1.3 \mathrm{GHz}$, which is still satisfying the system specifications. Overall, regarding the delay generation, the system performs as expected, meeting the requirements.

\subsection{Conclusion}

In this work we have presented the first hybrid integrated microwave photonic system and separately analyzed the performance of its essential building blocks. The realized system is an optical OBFN offering tunable true time delay of four signals from receiving microwaves antennas. The results show that every building block works as expected. However, the interplay of the building blocks revealed an issue of highest importance for future approaches, which is RF crosstalk between modulators and photodetectors fabricated on the same InP chip. This crosstalk was essentially the only severe limit for characterization of the device as a whole, while the optical performance was well within specifications. Several conclusions can be drawn as to what the most effective steps should be in the follow-up devices. Most urgent is to design the devices also for optimum electronic performance, specifically via properly shielded modulators, detectors and the according packaging. Moreover, having all the optical functionalities on one chip is sub-optimal also in terms of fabrication process. In fact, the fabrication processes for diode gains, modulators and photodetectors are different. Therefore, regrowth processes must be carried out in order to have them on one chip. The regrowth procedure is quite challenging, and the components obtained after the regrowth could have reduced quality.

A first step in this direction, in order to have optimum components and low RF crosstalk, is to place gain diode chips, modulators and detectors on separate chips to be attached to the TripleX chip.

Various improvements in the optical performance are to be addressed as well. The power of the on-chip hybrid laser should be raised to the $100-\mathrm{mW}$ level. This seems possible as based on prototype high-power amplifiers [51]. Another point of investigation is the undesired optical feedback into on-chip lasers, which is part of the program in a current EU research project [52]. Last, 
interface losses must be lowered, to have an efficient propagation of the optical signals among the different platforms. 
Hybrid integrated $4 x 1$ receiver equipped with ring resonators-based optical 


\section{CHAPTER 7 \\ HYBRID INTEGRATED 1X4 TRANSMITTER EQUIPPED WITH SWITCHED DELAY LINES-BASED OPTICAL BEAMFORMING NETWORK}

\subsection{Introduction}

In this chapter we present the first fully integrated MWP system, which is a $1 \mathrm{x} 4$ transmitter TTD-OBFN. Its working principle is based on stepwise TTD, called switched delay lines (SDL), as processing core, which will be described in section 7.3 .

The fully IMWP system presented here is based on hybrid integration of semiconductor and dielectric waveguide chips and is the result of an iterative process, in which several issues where empirically addressed at each iteration. During this process, the outcomes at each step of this iterative process have been inspected with growing insight to get a better understanding of the overall system and the way in which the individual components influence the performance of the RF-to-RF system.

In this chapter, the measurement results obtained by testing the IMWP OBFN demonstrator will be presented and used to conclude on what steps should be done for improving the next generation of hybrid integrated systems. The first demonstrator is possibly the most important milestone, already due to its "inspiring" impact in driving the field of IMWP, in the on-going iterative process towards high-performance MWP systems of all kinds, since, for the first time, it has been possible to measure the typical RF-to-RF system properties, 


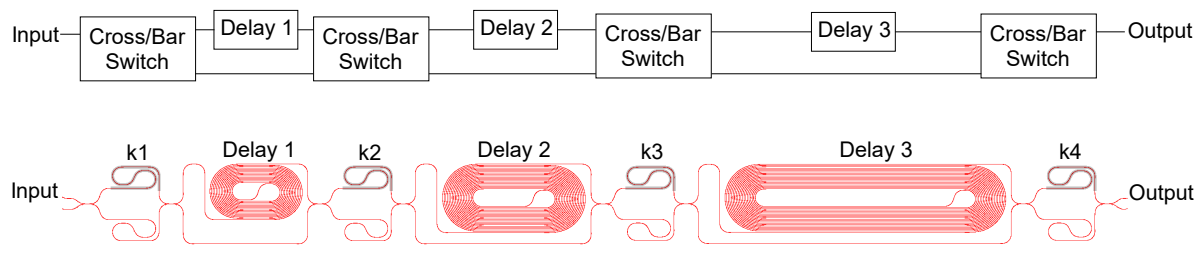

Figure 7.1: Top: Building blocks schematic for the switched delay line; Bottom: Waveguide layout of the first switched delay line where the parameters are highlighted

such as signal transfer, noise figure, and non-linearities.

\subsection{OBFN based on switched delay line}

We introduce here the structure of a SDL, which is the basic building block for the OBFN present in the demonstrator. A SDL comprises short and long paths between cross-bar switches, as shown in Figure 7.1. The cross-bar switches are implemented as tunable couplers (see section 3.3.0.2), and are labeled with $\kappa_{1}, \kappa_{2}, \kappa_{3}$, and $\kappa_{4}$ in the figure. The optical path length difference between the short and spiral-like long paths determines its contribution to the total time delay. The geometrical path length difference can be calculated from the desired time delay, $\Delta T$, as follows

$$
\Delta L=\frac{c \Delta T}{n_{g}}
$$

In particular, the long paths were designed such that the second delay is twice as much as the first delay, and the third delay is twice as much as the second one. The cross-bar switches of the SDL can be tuned to effectuate stepwise delays. The optical parameters, namely the power coupling coefficients required to route the optical signals through the desired delay, are listed in Table 7.1.

\subsection{Functional design and layouts}

A graphical impression of the full system is presented in Figure 7.2. The InP chip at the upper left contains an optical gain section which, together with the tunable feedback cavity arm based on RRs on the TripleX chip, provides a highly frequency-stable on-chip optical carrier. The generated light travels to an InP MZM chip to encode the incoming RF signal onto the optical carrier. In this case, an MZM was chosen to perform intensity modulation of the optical carrier, which is directly detected by the photodiode. Note that, in this case, there is no limitation in the bandwidth as compared to the system presented in 
Table 7.1: Power coupling coefficients needed to route the optical signals through the required stepwise delays, where a value of 1 indicates a cross-status while a value of 0 indicates a bar-status of the corresponding switch

\begin{tabular}{||c|c|c|c|c||}
\hline Delay & $k_{1}$ & $k_{2}$ & $k_{3}$ & $k_{4}$ \\
\hline \hline No Delay & 1 & 0 & 0 & 1 \\
\hline Delay 1 $(\bar{T})$ & 0 & 1 & 0 & 1 \\
\hline Delay 2 $(2 T)$ & 1 & 1 & 1 & 1 \\
\hline Delay 1 + Delay 2 $(3 \bar{T})$ & 0 & 0 & 1 & 1 \\
\hline Delay 3 $(4 \bar{T})$ & 1 & 0 & 1 & 0 \\
\hline Delay 1 + Delay 3 $(5 \bar{T})$ & 0 & 1 & 1 & 0 \\
\hline Delay 2 + Delay 3 $(6 \bar{T})$ & 1 & 1 & 0 & 0 \\
\hline Delay 1 + Delay 2 + Delay 3 $(7 \bar{T})$ & 0 & 0 & 0 & 0 \\
\hline
\end{tabular}

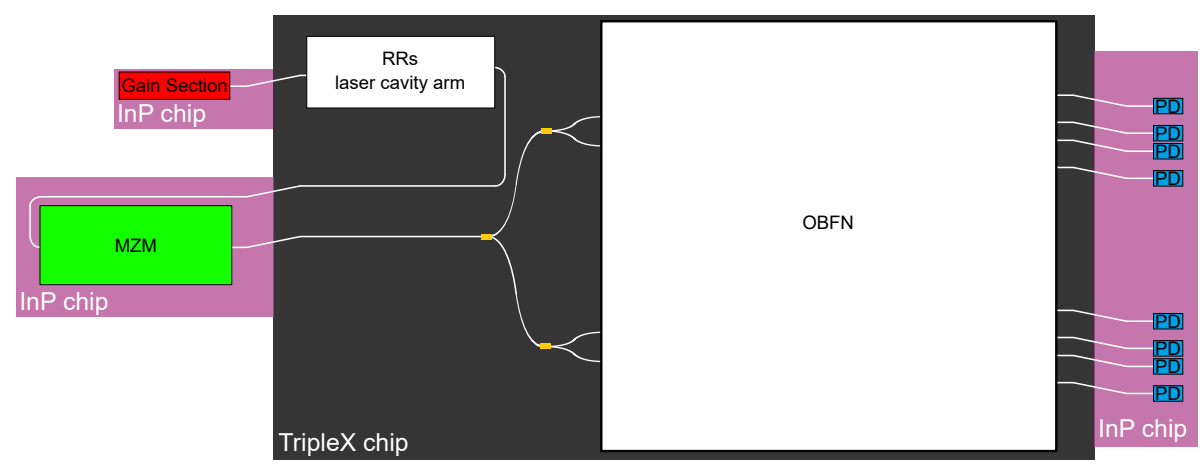

Figure 7.2: Functional design of the first demonstrator of the fully hybrid integrated OBFN system 

optical beamforming network

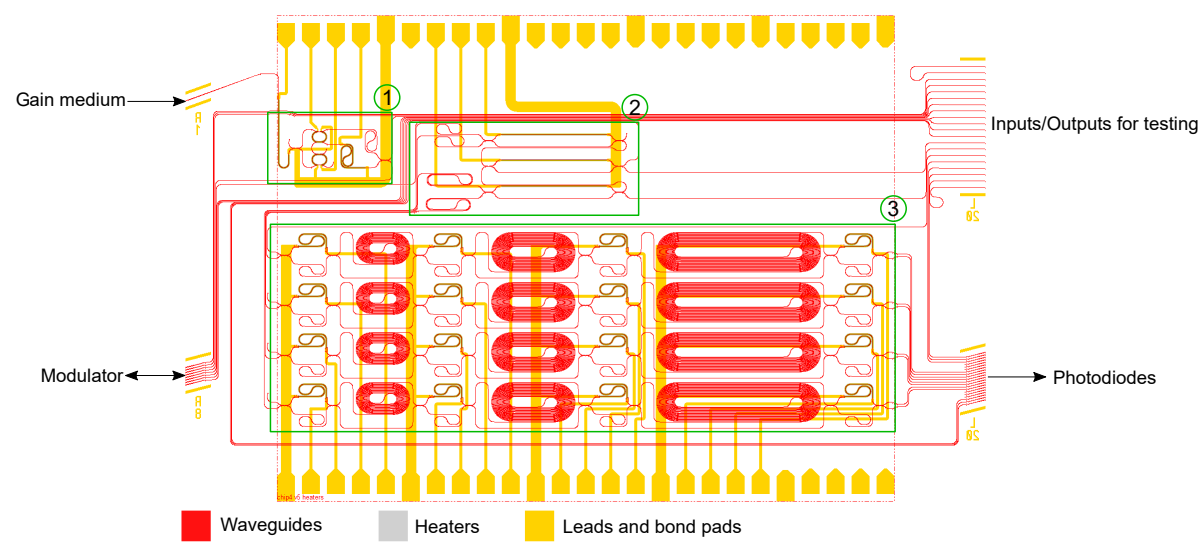

Figure 7.3: Waveguide layout of the TripleX chip. The functional building blocks are highlighted and labeled with different numbers: 1 for the RRs laser cavity structure, 2 for the $1 \mathrm{x} 4$ tunable splitter and 3 for the four SDLs.

chapter 6 (given by the RRs). When SDLs are used, both the side-frequencies will experience the same delay, and they can therefore both be kept.

The intensity-modulated light is guided then to the actual processing core of the IMWP system, which is the $1 \mathrm{x} 4$ OBFN based on SDLs, as described in section 7.2. The modulated light is split into four branches, which are the inputs to four SDLs. Each SDL has two outputs, which are both guided to photodiodes on an InP chip. Only one photodiode per SDL would be sufficient in a basic functional design, and we have used two photodiodes for back-up. All the passive components, namely the laser external cavity arm, the $1 x 4$ OBFN and path-compensation waveguides are integrated on a single chip realized using the TripleX technology $\left(\mathrm{Si}_{3} \mathrm{~N}_{4} / \mathrm{SiO}_{2}\right.$ technology). The active components, namely the laser gain medium, modulators and detectors, are fabricated using an InP platform as three separate chips. In fact, previous versions attempted an immediate integration of two or more active components on single chips but turned out to be less effective because of mutual perturbances. For example, RF crosstalk would be too high when modulators and detectors are on the same InP chip, as shown in section 6.6.4.1. Similarly, scattered light from a highpower gain section would saturate the photodetectors when placed together on a single InP chip. On the other hand, it becomes more challenging to integrate multiple InP chips with the TripleX chip.

\subsubsection{Delay line chip}

The actual waveguide layout of the TripleX chip is shown in Figure 7.3, where not only the waveguide structure is visible, but also the heaters used for thermooptical tuning, the electrical leads and bond pads. The functional build- 


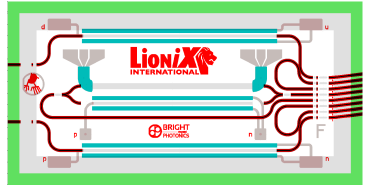

(a)
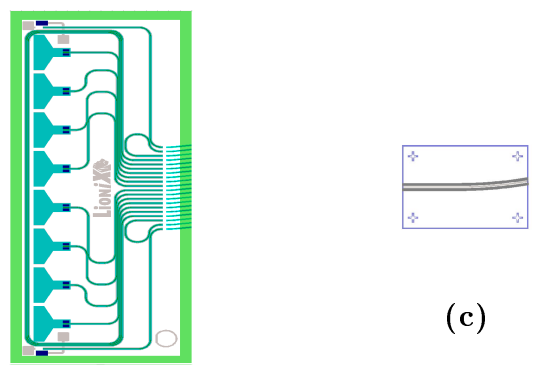

(c)

(b)

Figure 7.4: Waveguide layout of a) the MZM modulator, b) the 8-photodiodes array, and c) the gain chip diode

ing blocks presented earlier are highlighted and labeled with different numbers. Each functional block can be accessed for testing by the input/output waveguide ports at the upper right side if connected to a fiber array. The input/output waveguides are enclosed with waveguide loops which are used to monitor the chip-to-chip coupling loss during alignment. The TripleX chip dimensions are $1.6 \mathrm{~cm}$ in length and $0.98 \mathrm{~cm}$ in width. The waveguides are based on an asymmetric double-stripe (ADS) geometry, which shows a measured propagation loss of $0.1 \mathrm{~dB} / \mathrm{cm}$ [38]. One can refer to Figure 6.1, where a SEM picture of a typical ADS waveguide cross-section is shown. Moreover, the ADS geometry is beneficial for an optimized the coupling to external components such as fibers or semiconductor waveguide circuits.

\subsubsection{Modulator and photodiodes chips}

The layout of the InP modulator and detectors chips are shown in Figure 7.4(a) and Figure 7.4(b). The modulator and photodiode chips were fabricated by SMART Photonics. The modulator chip comprises a single MZM because the hybrid integrated system presented here is designed to work as an RF transmitter and therefore only one modulator is needed. The photodiode chip comprises eight detectors. Also, the InP chips are equipped with waveguide loops, to monitor the chip-to-chip insertion loss during alignment. These losses are highly important parameters that need to be determined since they influence the overall link gain of the system.

\subsubsection{InP gain chip}

The InP gain diode chip, whose schematic is shown in Figure 7.4(c), was fabricated by Fraunhofer HHI. The gain diode chip was designed to work in the C-band window $(1550 \mathrm{~nm})$. One side of the chip is anti-reflective coated, while 


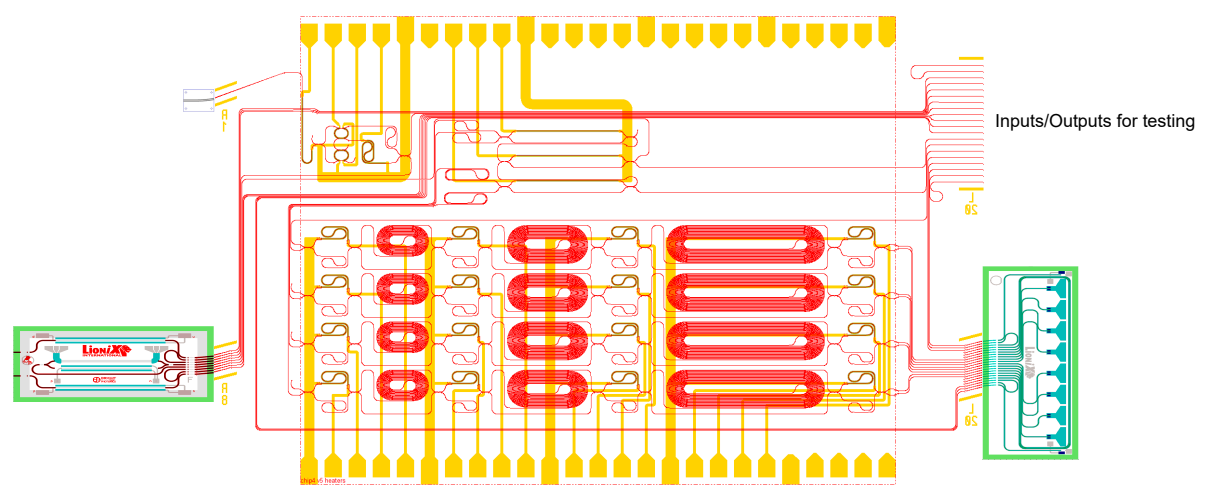

Figure 7.5: Waveguide layout of the $1 \mathrm{x} 4 \mathrm{OBFN}$ system, comprising the TripleX and the InP chips

the other side is highly reflective. The angle facet is 9 degrees to avoid backreflections.

Finally, the multi-chip system layout is shown in Figure 7.5, where the modulator chip and gain chip are placed on the left side, while the detectors chip is placed on the right side of the TripleX chip. The assembling and packaging has been performed by LioniX International.

\subsection{Fabrication and measurements of the hybrid integrated microwave photonic system}

A picture of the first fully hybrid integrated OBFN demonstrator is shown in Figure 7.6. The purpose of the picture is to show how the chips are coupled to each others and how dedicated PCBs are designed and connected to apply signals to the modulator, to collect signal from the detectors, to drive the thermo-tuning elements and to inject pump current into the gain medium. One can recognize the TripleX chip which is the central chip, at which the smaller InP chips are attached. The gain chip is attached on the top left side and is connected to the upper PCB for the current injection to generate light via the connector labeled $\mathrm{P} 1$ on the upper $\mathrm{PCB}$. On the bottom left side, the modulator is attached and the RF signal can be applied using the terminated $50 \Omega$ RF trace via the SMP connector, labeled P1 on the lower PCB. Since the modulator is an MZM type, a bias is needed in order to work in the linear regim, by biasing at its quadrature point (via the connector labeled P11 on the lower PCB). The chip with the 8-detector-array is placed on the bottom right side. The detectors can be accessed by the non-terminated $50 \Omega \mathrm{RF}$ traces via the SMP connectors labeled PD1 to PD8 on the lower PCB. The fiber array for testing purposes is placed on the top right side. Other connectors are present 


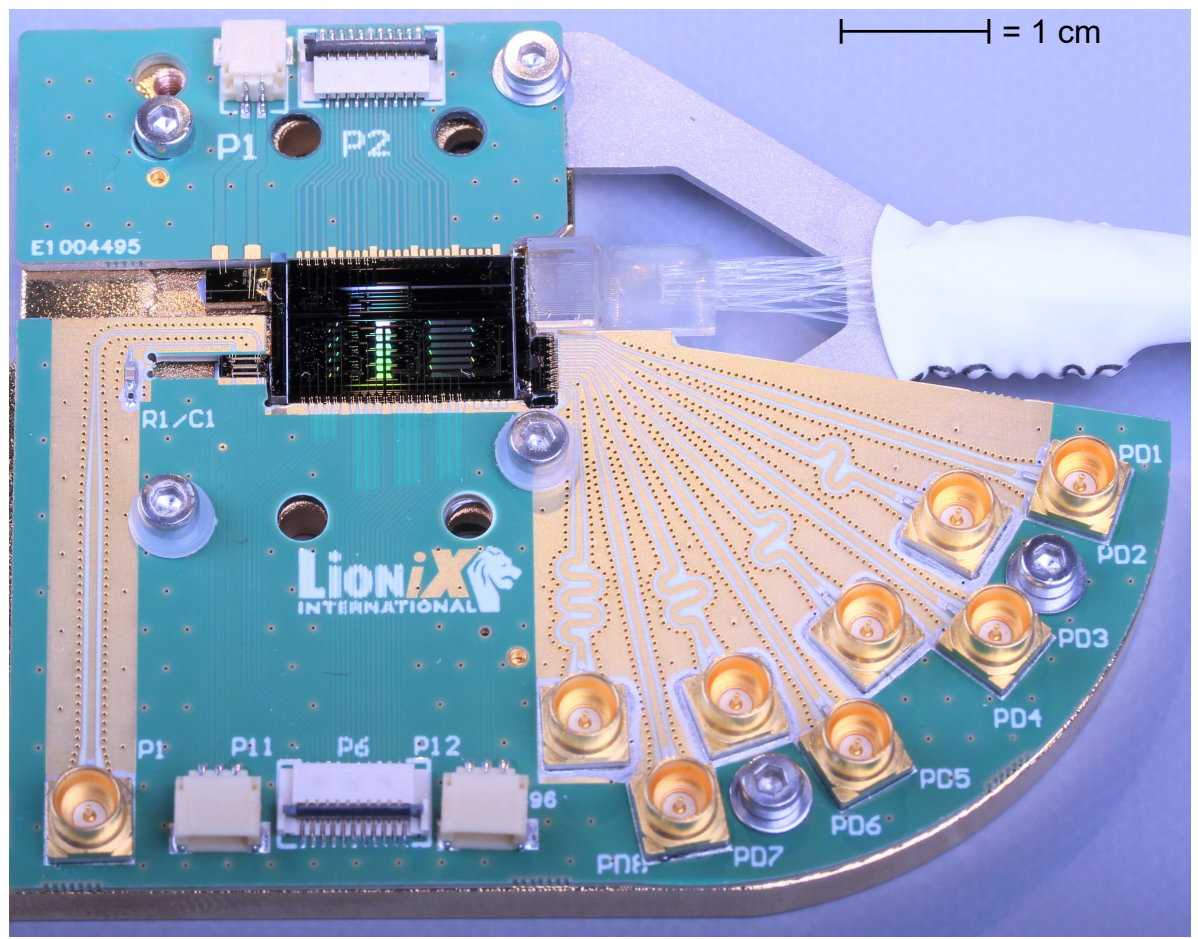

Figure 7.6: Picture of the first fully hybrid integrated OBFN demonstrator 


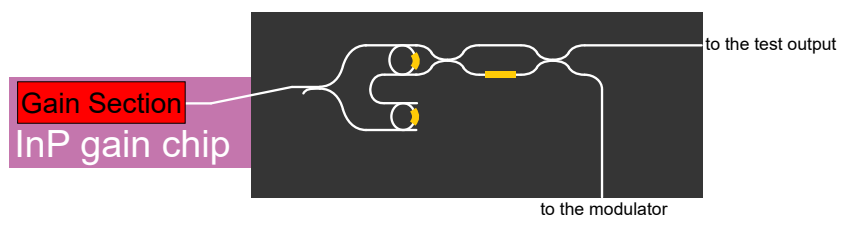

Figure 7.7: Functional layout of the hybrid integrated laser

on the upper and lower PCBs, namely the ones labeled P2 on the upper and $\mathrm{P} 6$ on the lower, and those are used for driving the thermo-tuning elements on the TripleX chip.

\subsubsection{Laser}

The first step for characterizing the integrated system is to verify the presence of a stable optical carrier. For this purpose, the RRs laser cavity arm is the first building block which is tuned until a frequency and power stable output is observed. For completeness, the functional layout of the hybrid laser is shown in Figure 7.7. In particular, the design of the laser cavity arm which makes use of RRs is shown, which comprises three 50/50 splitters and phase shifters acting on the light in the two RRs and in one branch of the Symmetric MZI. All the phase shifters are continuously tunable elements. That property makes the whole laser cavity tunable as is described in [39]. In particular, the laser central wavelength can be tuned by matched tuning of the roundtrip phase of the ring resonators, whose coupling coefficients to the bus waveguides are fixed. By tuning the phase shifter in the MZI, as described in section 3.3.0.1, the output of the laser can be either directed to the test fiber on the right, which is solely for evaluation of the output of the laser, or it can be directed to the modulator, for proper functioning of the system. The measured output power using the output test fiber connected to the optical power meter (Thorlabs PM100D) was $10.8 \mathrm{dBm}$ with an injected current of $300 \mathrm{~mA}$. The measured linewidth is as narrow as $50 \mathrm{kHz}$. This is extremally small when compared to other commercial integrated lasers, e.g., DFB lasers, that typically have a linewidth in the MHz-range. The RIN of such a laser has been measured and it is $-160 \mathrm{dBc} / \mathrm{Hz}$. RIN is an important parameter which influences the output noise of the entire system, as shown in section 2.2.2.

\subsubsection{Link gain}

Once the laser was found properly working, a characterization of the basic APL was performed. The basic building blocks forming an APL are the laser, the modulator and the detector. Figure 7.8 shows the setup for a measurement of the link gain. The internal laser is driven by a current source set to about 


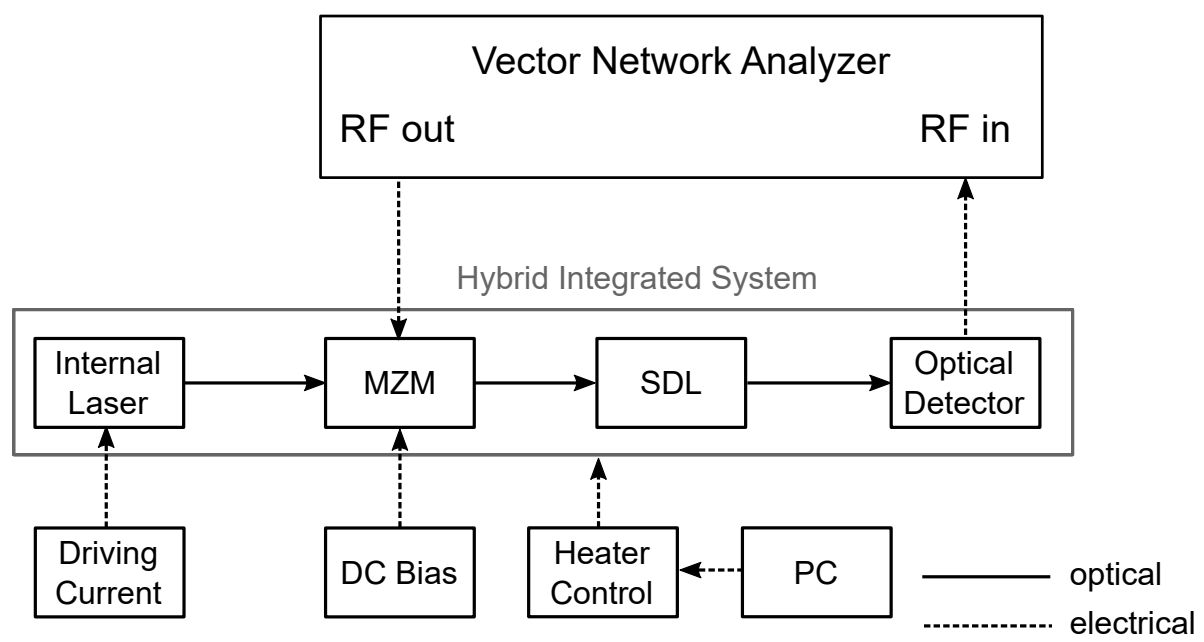

Figure 7.8: APL setup for link gain measurements

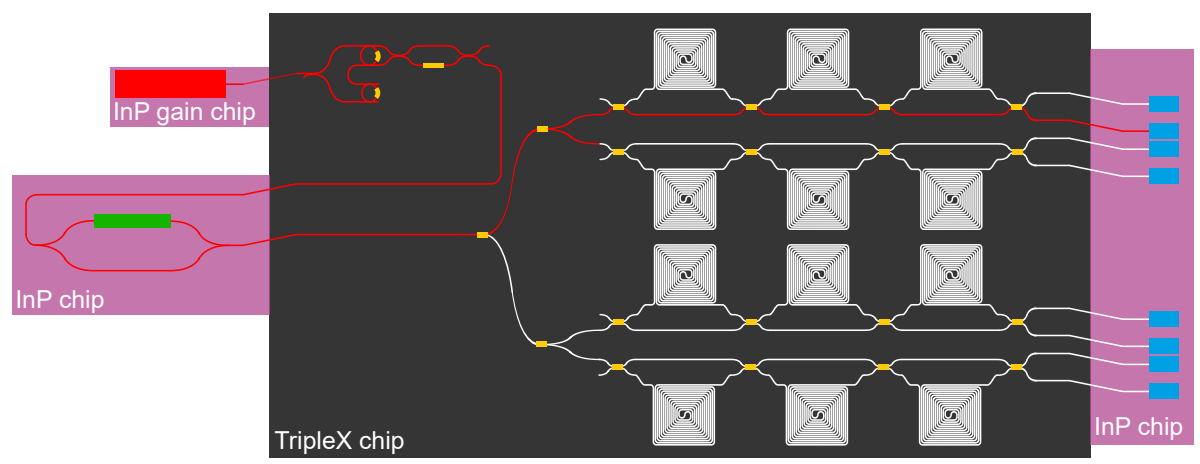

Figure 7.9: System functional design where the path used for the APL measurement is highlighted in red 


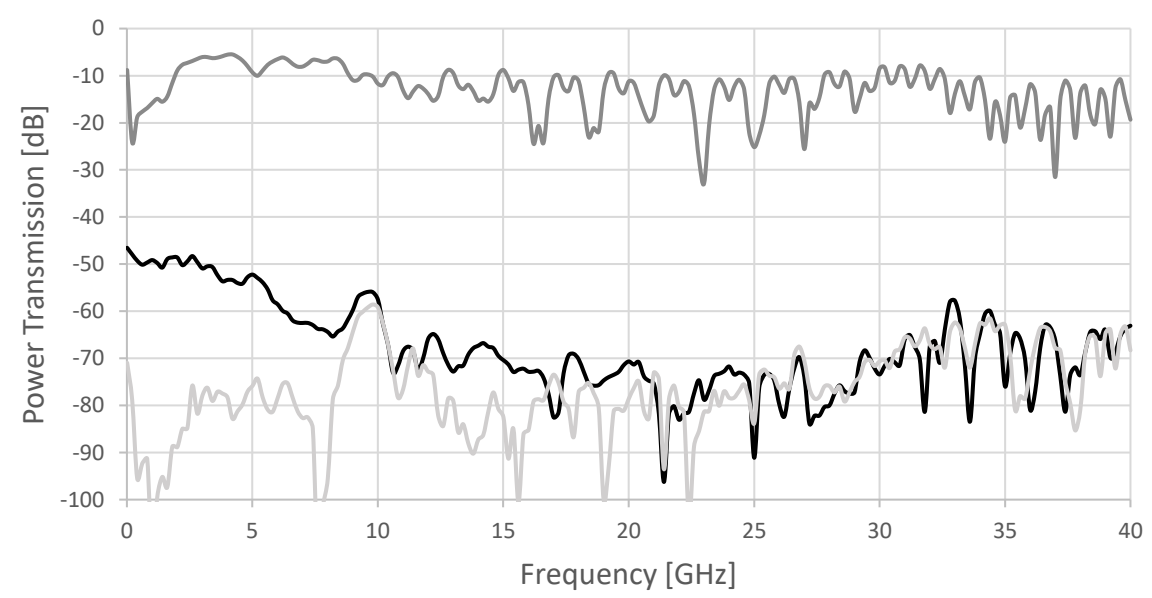

Figure 7.10: Link gain (black) measurement of the APL in dB, the input return loss (dark grey) in $\mathrm{dB}$, and the RF crosstalk (light grey) measured over a frequency range from $\mathrm{DC}$ to $40 \mathrm{GHz}$

$300 \mathrm{~mA}$ to generate $10.8 \mathrm{dBm}$ optical power from the laser. For an easier understanding of the multi-chip setting for the APL measurement, Figure 7.9 displays the total path from the laser to the photodiode as highlighted in red. The laser light is directed towards the MZM by adjusting the tunable coupler at the output of the laser cavity as described above. The MZM is biased at its quadrature point as described above. After modulation, the optical signal consists of three spectrally narrow lines, namely the optical carrier and two sidefrequencies, as is expected in the small signal approximation, where higher order side-frequencies are small when compared to the fundamental. The modulated light goes back to the TripleX chip. Here the $1 \times 4$ splitter, which consists of three tunable couplers, is adjusted such that all the power goes into a single SDL, for which we chose the first one in the experiment. The first SDL is tuned such that the signal propagates along the shortest path, as achieved by setting the tunable couplers to bypass the spiral-like delay lines. Ultimately, the optical signal is collected at the second photodiode from the top for opticalto-electrical conversion. The overall chip assembly is kept at a temperature of $30^{\circ} \mathrm{C}$.

An RF signal input, generated by a VNA in the form of a pure sine wave, is fed into the modulator. The RF output signal from the detector is collected and sent to the input port of the VNA. Comparison of the signals in the VNA yields the link gain parameter. The results of the measurement are displayed in Figure 7.10 and show a link gain of $-46 \mathrm{~dB}$ at low frequencies. The gain is approximately constant until $3 \mathrm{GHz}$. At higher frequencies the gain decreases linearly up to around $8 \mathrm{GHz}$. Around $8 \mathrm{GHz}$ the RF crosstalk becomes as high as the link gain. The background floor of the gain measurement is given by 
the RF crosstalk between modulator and detectors, also shown in Figure 7.10, which is indeed $-70 \mathrm{~dB}$. The main limitation in this specific case is the bandwidth of the MZM. Together with the link gain measurement, also the input return loss is shown. As was described in section 7.4, the $50 \Omega \mathrm{RF}$ trace to the modulator are matched to a load resistance of $50 \Omega$ for having impedance matching with the modulator, whose impedance is indeed $50 \Omega$. This characteristic can be observed by inspecting the input return loss, which is below -10 $\mathrm{dB}$ at low frequencies, while it stays around $-5 \mathrm{~dB}$ between $2 \mathrm{GHz}$ and $10 \mathrm{GHz}$ and then drops to $-10 \mathrm{~dB}$. Note that the input return should also be improved in order to have a constant value of $-10 \mathrm{~dB}$ within the operational bandwidth.

\subsubsection{Delay generation}

The measurement setup used for a characterization of the delay lines is the same as used for the link gain measurement, shown in Figure 7.8. Again, the first delay line was selected for the measurements, and the waveguide layout is depicted in Figure 7.1. In particular, the SDL was fabricated such that Delay 1 corresponds to $190 \mathrm{ps}$, Delay 2 to $380 \mathrm{ps}$ and Delay 3 to $760 \mathrm{ps}$. The maximum delay that can be achieved by the SDL, by tuning all the cross-bar switches in a bar state, is $1.33 \mathrm{~ns}$.

The measurements to prove the delay generation functionality are shown in Figure 7.11. Each curve corresponds to a combination of setting of the tunable couplers as indicated in Table 7.1. Figure 7.11(a) shows the RF power transmission in $\mathrm{dB}$. It can be seen that the frequency dependence of the transmission is approximately flat up to about $3 \mathrm{GHz}$. At higher frequencies, the various combinations of delays show a linear decrease in transmission (in the $\mathrm{dB}$ scale) up to $5 \mathrm{GHz}$. The power transmission amounts to around $-50 \mathrm{~dB}$ when the light goes through the shortest path, and to $-55 \mathrm{~dB}$ for the longest delay, the latter having an optical length on-chip of $39 \mathrm{~cm}$, and the corresponding pysical length in this case is $22.5 \mathrm{~cm}$. This is given by the optical length devided by the group index, $n_{g}$, which for asymmetric double-stripe waveguides in TripleX is 1.77. When carefully analyzing the loss and delay length, we find excellent agreement with the supposed low propagation loss of $0.1 \mathrm{~dB} / \mathrm{cm}$ of the chosen waveguide platform. The quick degradation in terms of frequency is found to be caused by the modulator and therefore it is expected that such OBFN can perform already much better when only improving the modulator. Furthermore, some ripples of about $100 \mathrm{MHz}$ repetition are visible. These can not be explained by optical interference and they are likely due to reflections in the coaxial cable because of the impedance mismatch at the photodiode.

In Figure 7.11(b) the measured group delay in ns is represented. The curve denoted with No Delay is due to the shortest path, that was highlighted in Figure 7.9. This path can be considered the reference path. The curves are separated by $190 \mathrm{ps}$, which is a confirmation of the desired delay generation, i.e., the averaged curves are at the expected values, as listed in Table 7.2. 


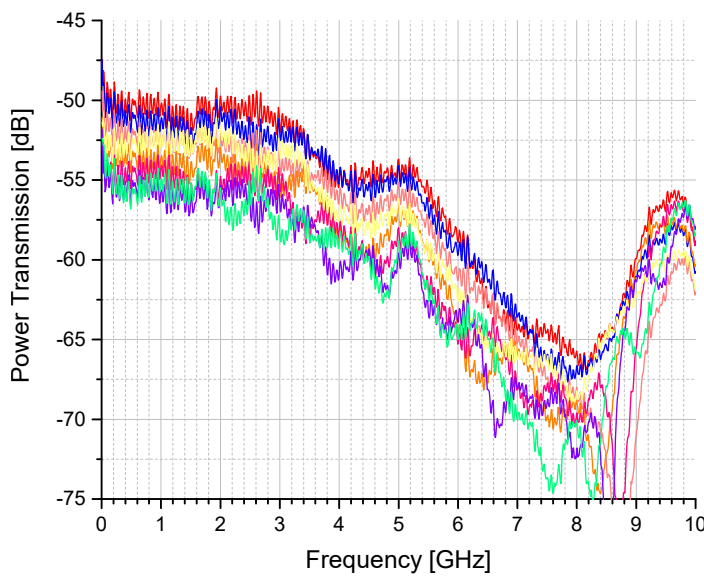

(a)

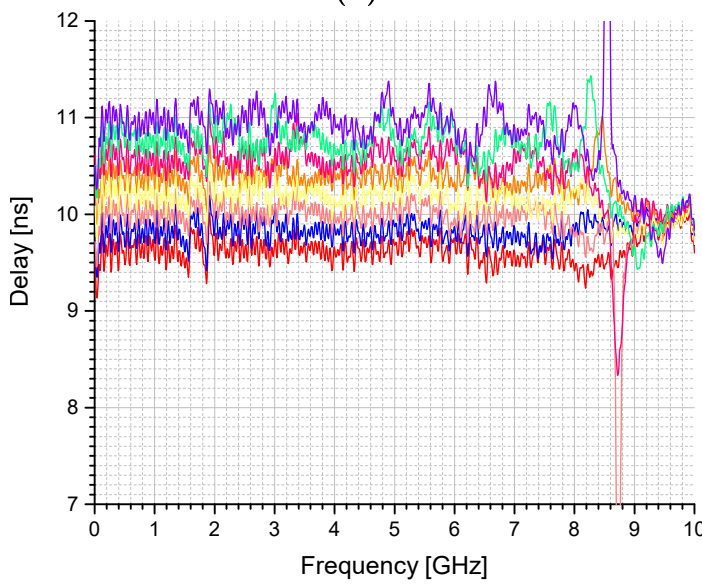

(b)

\begin{tabular}{|l|}
\hline No Delay \\
Delay 1 \\
Delay 2 \\
Delay 3 \\
Delay $1+$ Delay 2 \\
Delay $1+$ Delay 3 \\
Delay $2+$ Delay 3 \\
Delay $1+$ Delay 2 + Delay 3 \\
\hline
\end{tabular}

Figure 7.11: (a) Measured power transmission vs frequency with the different setting listed in Table 7.1; (b) accordingly measured group delay. 
Table 7.2: Desired delays compared to the reference delay. The difference between subsequent delays is $190 \mathrm{ps}$.

\begin{tabular}{||c|c|}
\hline Delay & Desired value \\
\hline \hline No Delay & Reference Delay \\
\hline Delay 1 & Reference Delay $+190 \mathrm{ps}$ \\
\hline Delay 2 & Reference Delay $+380 \mathrm{ps}$ \\
\hline Delay 1 + Delay 2 & Reference Delay $+570 \mathrm{ps}$ \\
\hline Delay 3 & Reference Delay $+760 \mathrm{ps}$ \\
\hline Delay 1 + Delay 3 & Reference Delay $+950 \mathrm{ps}$ \\
\hline Delay $2+$ Delay 3 & Reference Delay $+1.14 \mathrm{~ns}$ \\
\hline Delay 1 + Delay 2 + Delay 3 & Reference Delay $+1.33 \mathrm{~ns}$ \\
\hline
\end{tabular}

However, similarly to the power transmission response, also here a significant systematic ripple $( \pm 100 \mathrm{ps})$ is present.

Further investigations are required to point the cause of the ripple present in the power transmission and group delay responses. A closer analysis of unexpected characteristics encountered with the measurements at this point is difficult, i.e., when everything is integrated and assembled. In this configuration, it is extremely complex to uniquely separate different effects from each others, also because many non-optical components are involved, such as PCBs, RF traces and coaxial cables.

\subsubsection{Modulators}

The most relevant parameter to determine for a modulator is the half-wave voltage, $V_{\pi}$, which is the frequency-dependent voltage required to produce a $\pi$ phase shift. The value of the half-wave voltage in the stationary case, $V_{\pi, D C}$, will determine the bias voltage that has to be applied in order to have the modulator working at its quadrature point. The value of the half-wave voltage evaluated at higher frequencies, $V_{\pi, R F}$, influences the link gain of the overall system.

Two different methods were used to measure the half-wave voltage in the stationary case and the half-wave voltage at RF.

The first, $\left(V_{\pi, D C}\right)$, is measured by applying a sweeping DC voltage to the modulator and measuring the output optical power using an optical power meter. Looking at the maxima and minima, one can retrieve the bias voltage difference to have a maximum and a subsequent minimum. In our case, the $V_{\pi, D C}$ turned out to be $10.2 \mathrm{~V}$, therefore, a bias voltage equal to $5.1 \mathrm{~V}$ is required to have the modulator at its quadrature point.

In the case of the half-wave voltage at RF, the link shown in Figure 7.9 was used. The modulator is kept at its quadrature point. Such APL is known as 


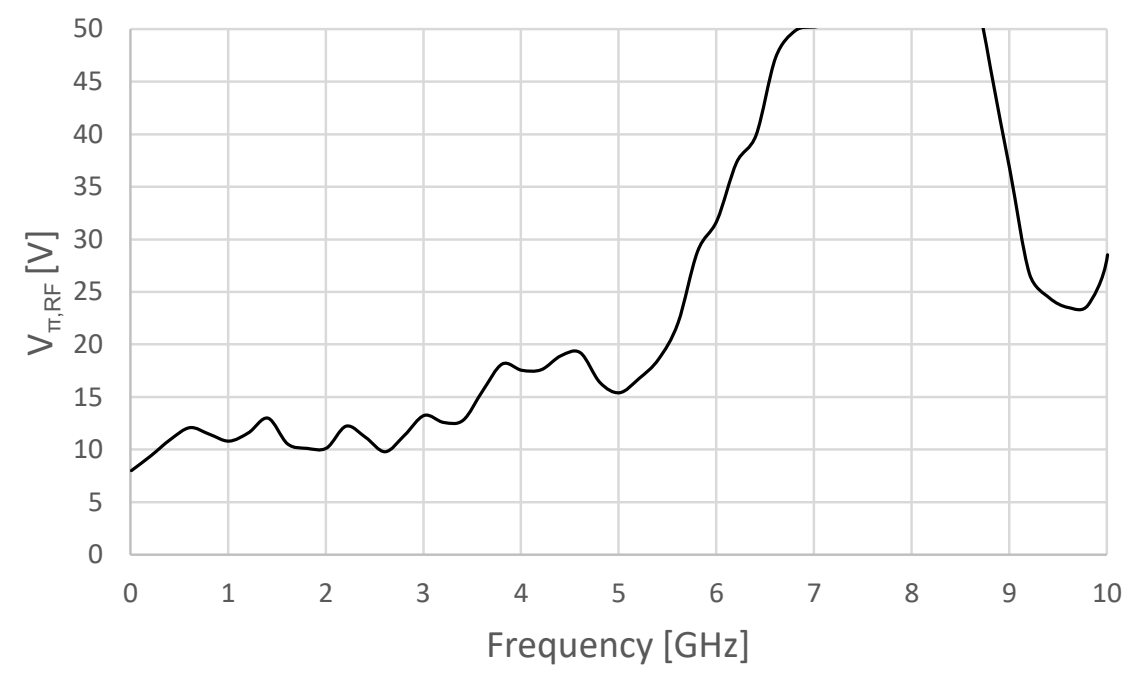

Figure 7.12: $V_{\pi, R F}$ of the MZM modulator shown in Figure 7.4(a) as a function of frequency

intensity modulation - direct detection, IM-DD. For this type of links, considering that in our case at the photodetector side there is not an impedance matching circuitry, the frequency-dependent link gain can be expressed as [15]:

$$
g(f)=\frac{I_{d c}^{2} \pi^{2} R_{i} R_{o}}{V_{\pi, R F}^{2}}
$$

where $I_{d c}$ is the average current at the photodiode, $R_{i}$ and $R_{o}$ are the input and the output load resistors, respectively. In our case, the average photocurrent was measured to be $260 \mu \mathrm{A}$. By measuring the link gain, upon knowing the parameters just described, it is possible to retrieve the frequency-dependence of the half-wave voltage at $\mathrm{RF}, V_{\pi, R F}$, of the modulator expressed as:

$$
V_{\pi, R F}(f)=\sqrt{\frac{I_{d c}^{2} \pi^{2} R_{i} R_{o}}{g(f)}} .
$$

In Figure 7.12 the $V_{\pi, R F}$ versus frequency is shown. It can be seen that $V_{\pi, R F}$ is less than $10 \mathrm{~V}$ for very low frequencies $(<200 \mathrm{MHz})$, while it stays at about $10 \mathrm{~V}$ for frequencies of up to $3 \mathrm{GHz}$ and then it increases linearly in the range up to $6 \mathrm{GHz}$. For higher frequencies, the link gain becomes lower than the RF crosstalk, and therefore the $V_{\pi, R F}$ cannot be considered at those frequencies. It is important to have the $V_{\pi, R F}$ as low as possible since, the higher $V_{\pi, R F}$ is, the lower the link gain is, for the same link setup and the same average current. In our case, a value of $10 \mathrm{~V}$ for the half-wave voltage in the frequency range of interest is certainly too high to have a good overall RF-to-RF link. 


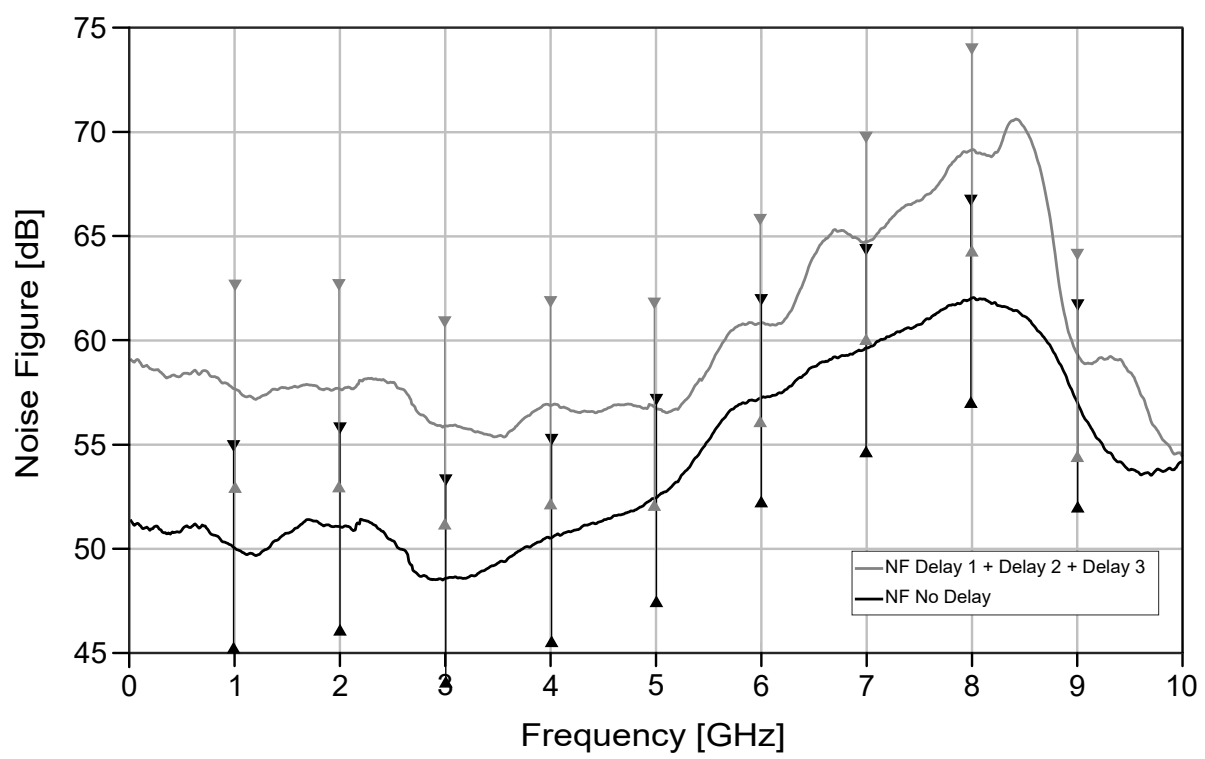

Figure 7.13: Noise Figure measurements of the first SDL for the shortest path in yellow and the longest path in blue

\subsubsection{Noise and non-linear distorsions}

The noise factor, $F$ (or the noise figure, $N F$, which is the noise factor expressed in $\mathrm{dB}$ as shown in section 2.2.2), is an absolutely central parameter used to characterize the ability of a system to process low-level signals. An IMWP system externally looks like a standard electronic system and, similarly, there will be components in it that add noise and will tend to obscure weak signals. Analogously, the noise factor is defined as the ratio of the electronic signal-to-noise ratio at the input $\left(S N R_{i n}\right)$, where the input noise power is due to thermal noise at room temperature, and the electronic signal-to-noise ratio at the output $\left(S N R_{\text {out }}\right)$ of the IMWP system, assuming a matched input source. Practically, we have measured the noise figure of the system with a VNA. The technique uses the continuous wave signal from one of the VNA sources. Different detectors in the VNA measure the "signal" respectively and the "signal+noise" output power of the device under test, which in our case is the hybrid IMWP system. The measurements results are given in Figure 7.13. The two curves represent two measurements, one for the shortest path, (named No Delay in Table 7.1), and one for the longest path, (named Delay $1+$ Delay $2+$ Delay 3 in Table 7.1).

The measured values of the noise figure vary from $51 \mathrm{~dB}$ to $59 \mathrm{~dB}$, with an error of \pm 5.75 . The error estimation was evaluated by a commercial tool, where one can specify the used setup for the noise figure measurement and the tool calculates the RMS of the error value. The expected value for the shortest 


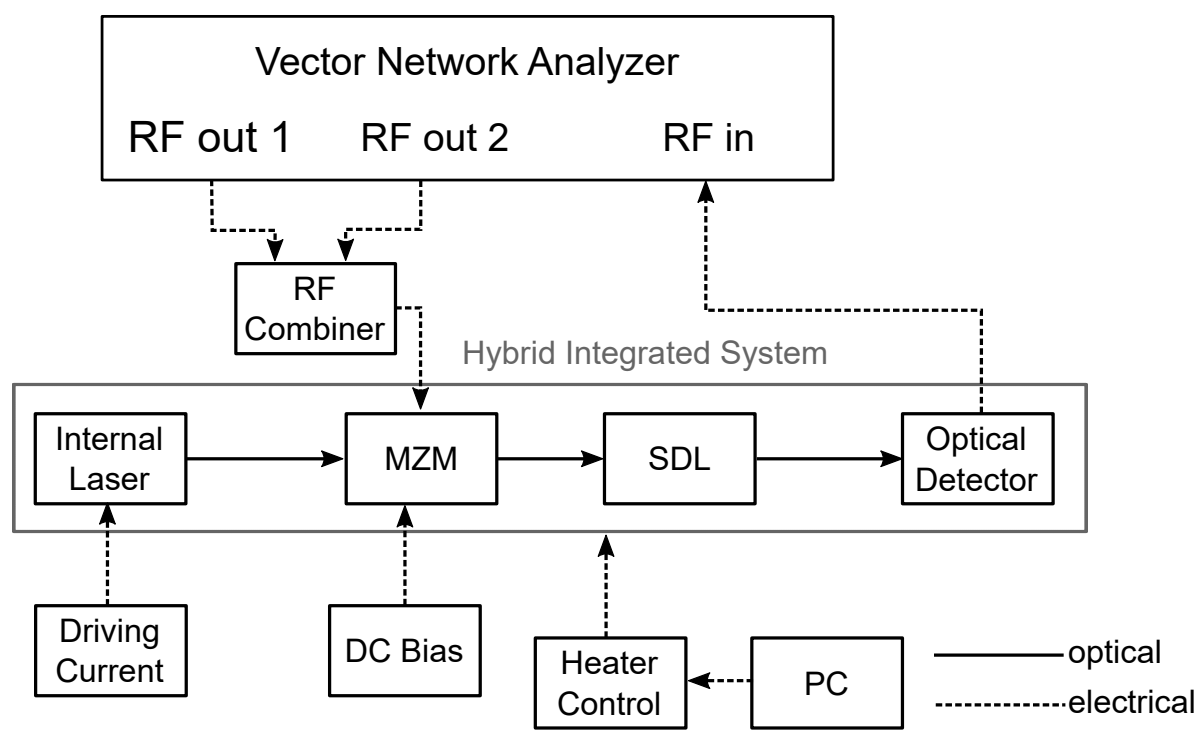

Figure 7.14: Setup for the output intercept point of the third order (OIP3) measurement by using the VNA

path, from (2.30), is $49.6 \mathrm{~dB}$. The power spectral density can be calculated from (2.24), considering that the measured average photocurrent was $260 \mu \mathrm{A}$. Therefore, the value is calculated to be $N_{\text {out }}=-170.694 \mathrm{dBm} / \mathrm{Hz}$.

There are two options to reduce the uncertainty on the $N F$ measurement. The first possibility is using a pre-amplifier, placed before the VNA receiver input, to lessen the receiver noise figure. However, a preamplifier with certain specifications, such as a gain in the order of $10 \mathrm{~dB}$ and working for a wide range of frequencies (from $1 \mathrm{GHz}$ to $20 \mathrm{GHz}$ ) is highly expensive. The second possibility is to increase the link gain of the overall system, and since we believe that the next generation of hybrid IMWP systems will perform much better, we decided to wait for the next available assemblies. The required and feasible improvements are addressed in the final chapter of this thesis.

The second measurement performed in the context of noise and distorsion was the characterization of non-linearities in the APL depicted in Figure 7.9. The objective of this measurement is to determine the $\mathrm{SFDR}_{3}$ (one can refer to section 2.2.3.2), which is commonly used to derive the input power range in which the received signal can be detected in the presence of noise and amplified without non-linear interferences.

The measurement setup is shown in Figure 7.14. Here, two RF signals, also named two tones (named RF out 1 and RF out 2), with equal power but different frequencies, which are $1.0 \mathrm{GHz}$ and $1.1 \mathrm{GHz}$, being $100 \mathrm{MHz}$ apart from each other, are generated by the VNA. The two RF signals are combined 


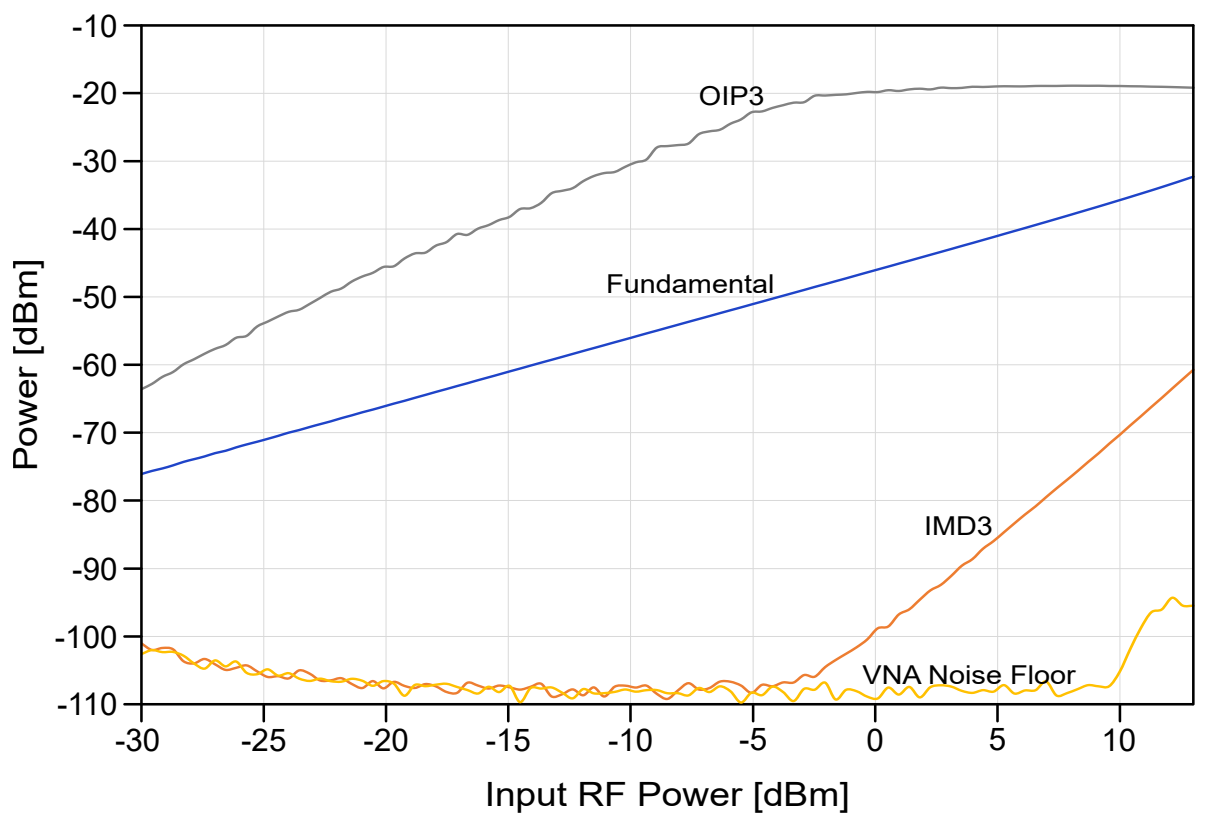

Figure 7.15: Measurement results for the non linear distorsions characterization. In blue the fundamental power (whose slope is 1 ) with a frequency of $1 \mathrm{GHz}$ and increasing power is visible, in orange the IMD3 product power (whose slope is 3 ) is visible, in yellow the noise floor of the VNA and in gray the OIP3, all versus the input power in $\mathrm{dBm}$

by an RF combiner to be used as input to the MZM. The link is brought into operation and the SDL is set as shown in Figure 7.9. The RF output signal from the photodetector is then fed into the VNA, such that the fundamental $\mathrm{RF}$ power (at $\mathrm{RF}_{1}$ and at $\mathrm{RF}_{2}$ ) as well as the third-order intermodulation distorsion (IMD3) power (at $2 \mathrm{RF}_{1}-\mathrm{RF}_{2}$ and at $2 \mathrm{RF}_{2}-\mathrm{RF}_{1}$ ) can be measured, as shown in section 2.2.3.2. The input power of the two signals generated by the VNA is swept between $-30 \mathrm{dBm}$ and $13 \mathrm{dBm}$.

In Figure 7.15 the results of the measurement are shown. Here, it can be seen that the output power of the fundamental tone (at $1 \mathrm{GHz}$ ) increases from -75 $\mathrm{dBm}$ to approximately $-30 \mathrm{dBm}$, when the input power is increased from -30 $\mathrm{dBm}$ to $-13 \mathrm{dBm}$. The yellow curve represents the noise floor of the VNA. When the input power is sligthly more than $-3 \mathrm{dBm}$, the intermodulation product IMD3, in orange, rises above the noise floor. In gray, the OIP3 is shown, which settles at $-18.7 \mathrm{dBm}$. The extracted input intercept point is $27.3 \mathrm{dBm}$. Using Figure 7.15 to evaluate the $\mathrm{SFDR}_{3}$ would lead to a mistaken value. The error is due to the fact that the noise of the hybrid IMWP system is lower than the noise floor of the VNA. Therefore, using the noise floor of Figure 7.15 yields to a value for the $\mathrm{SFDR}_{3}$ that is lower than the actual value. Our approach 


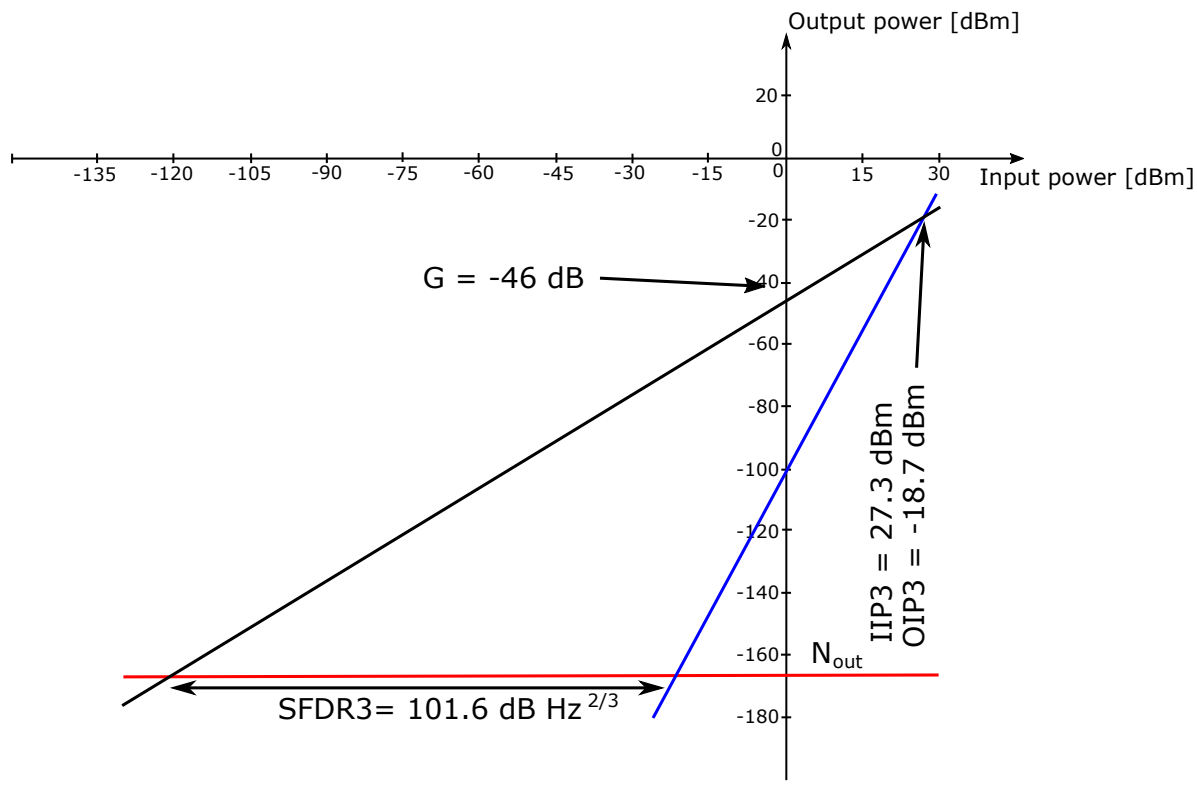

Figure 7.16: Extracted SFDR and intercept point between OIP3 and IIP3. The black line is the fundamental response. The blue line is the IMD3 and the red line is the calculated output noise

to determine the $\mathrm{SFDR}_{3}$ was to extrapolate the curves of the fundamental and the IMD3 from the measurements to evaluate the OIP3 and the input intercept point of the third order (IIP3) and to calculate the power spectral density from (2.24), considering that the measured average photocurrent was $260 \mu \mathrm{A}$. In Figure 7.16, the extrapolated curves of the fundamental, the IMD3 and the output noise PSD are shown. The output noise power spectral density is calculated as $-170.694 \mathrm{dBm} / \mathrm{Hz}$. Therefore, the $\mathrm{SFDR}_{3}$ is calculated from (2.41) or (2.42) and has a value of $101.6 \mathrm{dBm}$ in $1 \mathrm{~Hz}$ bandwidth.

\subsection{Discussion and conclusion}

In this chapter we have presented the very first fully hybrid integrated TTD OBFN system, where the OBFN is based on SDLs. The total system consists of three InP chips to achieve the active functionalities and one TripleX chip, which is the processing core of the system. The different basic building blocks have been inspected, such as the hybrid laser providing an average power of $10.8 \mathrm{dBm}$, the MZM with respect to the half-wave voltage, $V_{\pi, R F}$, and the link gain for the basic APL. Finally, the delay generation functionality was proven, which showed good agreement with the expected values. 
Table 7.3: Performance summary the OBFN based on SDLs, compared to the desired values for better performing APL

\begin{tabular}{|c|c|c|c|}
\hline Parameter & Symbol & Value & Desired achievable value \\
\hline Link Type & & IM-DD & IM-DD \\
\hline Laser Power & $P_{0}$ & $12 \mathrm{~mW}(10.8 \mathrm{dBm})$ & $40 \mathrm{~mW}(16 \mathrm{dBm})$ \\
\hline Relative Intensity noise & RIN & $<160 \mathrm{dBc} / \mathrm{Hz}$ & $<160 \mathrm{dBc} / \mathrm{Hz}$ \\
\hline Frequency & $f$ & $1 \mathrm{GHz}$ & $12-40 \mathrm{GHz}$ \\
\hline Half-wave voltage modulator & $V_{\pi, R F}$ & $8.5 \mathrm{~V}$ & $<3 \mathrm{~V}$ \\
\hline Responsivity photodetector & $R_{p d}$ & $0.8 \mathrm{~A} / \mathrm{W}$ & $0.9 \mathrm{~A} / \mathrm{W}$ \\
\hline Average photocurrent & $I_{d c}$ & $260 \mu A$ & $3 \mathrm{~mA}$ \\
\hline Optical Link Loss & $\mathrm{L}$ & $12.8 \mathrm{~dB}$ & $11 \mathrm{~dB}$ \\
\hline No matched Load at photodiode & & & \\
\hline RF link gain & $\mathrm{G}$ & $-46 \mathrm{~dB}$ & $-16 \mathrm{~dB}$ \\
\hline Output Intercept Point & OIP 3 & $-18.7 \mathrm{dBm}$ & $2.55 \mathrm{dBm}$ \\
\hline Input Intercept Point & IIP3 & $27.3 \mathrm{dBm}$ & $18.55 \mathrm{dBm}$ \\
\hline Output noise power spectral density & $N_{\text {out }}$ & $-170.684 \mathrm{dBm} / \mathrm{Hz}$ & $-160 \mathrm{dBm} / \mathrm{Hz}$ \\
\hline Noise Figure & NF & $49.6 \mathrm{~dB}$ & $30 \mathrm{~dB}$ \\
\hline Spurious Free Dynamic Range & SFDR & $101.6 \mathrm{~dB} \cdot H z^{2 / 3}$ & $108.4 \mathrm{~dB} \cdot H z^{2 / 3}$ \\
\hline
\end{tabular}

Overall, the system functions as expected. However, various improvements are to be addressed to have a better performing IMWP system.

In Table 7.3 we have collected all the meaningful measured parameters which describe the presented IMWP system, together with the desired achievable values, to have a better performing link. Comparing the last two columns of the table, it is easy to deduce the necessary improvements.

First of all, the optical power provided by the hybrid laser has to be raised. Based on the current gain diodes, an optical power of $40 \mathrm{~mW}$ seems to be achievable. The RIN should be as low as possible, and we find that the RIN of the hybrid integrated laser is already pretty low, but research is still on-going.

We have already mentioned that there is a severe bandwidth limitation, which is given by the modulator. Therefore, different types of modulators have to be investigated, which would fit the requirements on the bandwidth, especially for the higher frequencies such as 20 and $30 \mathrm{GHz}$, and on the halfwave voltage at $R F$, which needs to be in the order of few volts.

In the table we compared the performance of the available system presented in this chapter with the performance of a link considering better components, mantaining the same type of modulation (IM), in order to show the impact of the improvemenets. However, one could also use an alternative modulation option, i.e., phase modulation, as done in the previous chapter. In this case, the phase modulator can be combined with a $R R$ to rotate the phase of the carrier, such that the spectrum resembles the intensity-modulated one, to be able to transform phase variation into intensity modulation of the optical carrier, keeping both the sidefrequencies. This would increase the link gain of 6 dB. More details can be found in [53].

Another possible improvements is in the link loss, which is the combination of the chip-to-chip coupling losses and propagation losses. Propagation losses are actually already extremely low, especially in the TripleX platform. From this point of view, the designing approach in our case has been to have the majority of the passive functionalities, such as delay lines, splitters and path- 
compensation waveguides on the TripleX, to avoid significant path-length in the InP chips. Instead, the chip-to-chip coupling losses have a bigger margin of improvements, and there is an on-going study in this sense to match the beamprofile of the two platforms by a "smart" tapering of the TripleX waveguides at the interfaces.

Concerning the photodetection, it can be improved by using photodiodes with higher responsivity. Moreover, instead of using an impedance matching circuitry, as it is in most of the cases, one could replace it with a transimpedance amplifier, which is used to convert current to voltage.

Once all these values are as desired, the rest of the parameters are automatically calculated, namely the link gain would have a value of $-16 \mathrm{~dB}$. The noise figure is then expected to be $30 \mathrm{~dB}$, and the SFDR is expected to be 108.4 $\mathrm{dBm}$. Those values tell that with the aforementioned improvements, an APL shows a comparable performance as the purely electronic solutions. 


\section{CHAPTER 8}

CONCLUSION AND OUTLOOK

\subsection{Conclusion}

In this thesis we have explored and proved the feasibility of novel integrated microwave photonic systems, which can be used as optical beamforming networks for antenna arrays, based on hybrid integration exploiting the combined properties of $\mathrm{InP}$ and $\mathrm{Si}_{3} \mathrm{~N}_{4} / \mathrm{SiO}_{2}$ as waveguides platforms.

Parallel to the hybrid integration, we have also investigated and demonstrated complex signal processing functionalities obtained by combining basic building blocks, such as splitters, combiners, ring resonators and phase shifters on the ultra low-loss $\mathrm{Si}_{3} \mathrm{~N}_{4} / \mathrm{SiO}_{2}$ waveguide platform, and that can eventually be considered as a part to be integrated in the processing core of a more generalized integrated microwave photonic system.

In Chapter 5, we have experimentally demonstrated an $8^{\text {th }}$-order MWP filter fabricated on $\mathrm{Si}_{3} \mathrm{~N}_{4} / \mathrm{SiO}_{2}$ waveguide platform, using a single-stripe waveguide structure, which ensures an extremely low-loss propagation, as low as 0.01 $\mathrm{dB} / \mathrm{cm}$, which could be used as multiplexer or demultiplexer in a satellite payload to filter single or a combination of channels. The filter comprises 8 coupled ring resonators, forming a structure known in literature as coupled resonators optical waveguide (CROW) [4]. The drop-port of such a filter can be used as passband filter, whereas the through-port can be used as stopband filter. The great advantage of having the CROW filter on a $\mathrm{Si}_{3} \mathrm{~N}_{4} / \mathrm{SiO}_{2}$ substrate is that the filter is completely reconfigurable, meaning that all the optical parameters, i.e., couplers and phase shifters, are tunable. As the frequency responses of the drop- and through- port depend on these optical parameters, this means that ultimately the filter can be reconfigured. The choice of the most suitable 
platform, namely $\mathrm{Si}_{3} \mathrm{~N}_{4} / \mathrm{SiO}_{2}$, has been crucial, since having an optical filter with bandwidth in the order of MHzs requires very long path lengths. The experimental verification showed a drop-port power transmission response with a flat-top profile passband filter, with a bandwidth of $72 \mathrm{MHz}$, an insertion loss of $13 \mathrm{~dB}$ and a stopband rejection of at least $51 \mathrm{~dB}$. The through-port power transmission response functions as stopband filter with adjustable bandwidth, which is determined by the number of rings brought into operation. The center frequency of the two filters can be changed by tuning the phases of the ring resonators.

Chapter 6 and Chapter 7 show both the state-of-the-art of hybrid integrated microwave photonic systems. Both systems are designed to be the feeding networks to antenna arrays for performing true-time delay operation. The difference is that the system presented in Chapter 6 is a receiver based on ring resonators to provide a continously-tunable delay, while in Chapter 7 the delay generation for a transmitter is based on switched delay lines, meaning that the possible delay values are stepwise. Besides the way the delay can be generated, the two systems are the milestones for the on-going process whose aim is to have a competitive integrated microwave photonic system which can be actually part of a telecommunication link.

Therefore, the essential building blocks for both systems were separately analyzed, together with the most important RF-to-RF parameters which fully describe an analog photonic link. The results obtained from the measurements of the system in Chapter 6 show that every building block works as expected. However, the interplay of the building blocks revealed an issue of highest importance for future approaches, which is RF crosstalk between modulators and photodetectors fabricated on the same InP chip. The conclusion was that the most urgent step was to design the active devices on separate InP chips. Moreover, various improvements in the optical performance are to be addressed as well. Certainly, the power of the on-chip hybrid laser should be raised to approaching the $100-\mathrm{mW}$-level. It was very difficult to draw qualitative conclusions on required improvements for other parameters since it was not possible to reliably measure parameters such as link gain, noise figure, and non-linearities.

In Chapter 7 we have presented the very first fully hybrid integrated true-time delay OBFN system. In this device the OBFN is based on switched delay lines. The total system consists of three InP chips to achieve the active functionalities and one TripleX chip, which is the processing core of the system. The different basic building blocks have been inspected, such as the hybrid laser providing an average power of $10.8 \mathrm{dBm}$, the MZM with respect to the half-wave voltage, $V_{\pi, R F}$, and the link gain for the basic analog photonic link. Finally, the delay generation functionality was proven, which showed good agreement with the expected values.

Overall, the system functions as expected. However, various improvements are to be addressed to have a better performing integrated microwave pho- 
tonic system. In the next section, some directions for further reasearch will be suggested.

\subsection{Outlook}

In this section, some practical suggestions are given in order to come to a hybrid integrated microwave photonic system which could effectively be used in a telecommunication link. It is important to highlight that the starting point is to realize hybrid integration between $\mathrm{InP}$ and TripleX, the former being a very reliable and mature platform and the latter being characterized by high maturity and extremely low propagation loss, necessary for having high delays or optical filters with bandwidths comparable with common microwave filters. However, even if the hybrid integration seems to be particularly promising, various improvements are to be addressed. In particular, looking at the typical RF-to-RF metrics, the link gain, the noise figure and the spurious-free dynamic range need to be improved to better values in the next step of development.

A most important point is to have a higher laser power in the order of 16 $\mathrm{dBm}$ at least. This seems to be feasible, based on the current available gain diodes. Concerning the modulation, irrespective of the type of modulation, modulators with lower half-wave voltage are required, since lowering this value increases the overall link gain. Moreover, other modulation techniques should be implemented and compared. A promising technique is based on phase modulation combined with a ring resonator, to have phase modulation to intensity modulation conversion, by rotating the phase of the optical carrier, as shown in [53].

When the laser power will be higher, the half-wave voltage of the modulators lower, it will be necessary to consider high power handling photodetectors, to avoid severe intermodulation distortions.

Besides building block improvements, it is also important to minimize the losses due to the chip-to-chip coupling. Several European projects and international industries are focusing their work on having a reliable automatic assembling process, to minimize errors due to manual alignment.

Another important aspect that is to be addressed is about the tuning technique. It was shown that, among the others, the possibility of having all the devices completely tunable, and hence reconfigurable, is one of major strengths of the MWP systems. The standard technique that was used in all the devices presented in this thesis was based on thermo-optical tuning, which requires a significant amount of power. However, in a satellite, or in general in any antenna, power is often a constraint. Therefore, there is an on-going research in order to explore other tuning techniques, with higher efficiency, requiring less power. A promising method seems to be the one based on deposition of thin layers of piezoelectric material to be used as stress-inducer to the waveguides 
[14].

Last, for future real applications, an efficient algorithm would be needed in order to tune automatically all the building blocks, such as couplers, RRs and filters present in the IMWP system, possibly via remoting, such that the same system does not need to be bound to a specific telecommunication link, but can be adjusted according to the need and re-used for meeting different requirements. An important step was already done in this direction, applying a customized algorithm as shown in [50].

To conclude, when all the improvements are addressed, the performance of integrated microwave photonic systems will become comparable, or even compete favourably with entirely electronic counterparts. In this way, the existing telecommunications infrastructures can be, whenever needed, enhanced by introducing IMWP systems in the most critical parts. 
[1] S.E. Schoenherr. The digital Revolution. 2004. URL: https : / / web . archive.org/web/20081007132355/http://history . sandiego.edu/ gen/recording/digital.html.

[2] K. C. Kao and G. A. Hockham. "Dielectric-fibre surface waveguides for optical frequencies". In: Proceedings of the Institution of Electrical Engineers 113.7 (July 1966), pp. 1151-1158. ISSN: 0020-3270. DOI: 10.1049/ piee.1966.0189.

[3] Richard Williamson and Ronald Esman. "RF Photonics". In: J Lightwave Technol 26.9 (2008), pp. 1145-1153. ISSN: 0733-8724. DOI: 10.1109/JLT. 2008.923627.

[4] J. Capmany and N. Dalma. "Microwave Photonics combines two worlds". In: Nat Photon 1 (2007), pp. 319-330. DOI: 10.1038/nphoton.2007.89.

[5] S. Iezekiel et al. "RF Engineering Meets Optoelectronics: Progress in Integrated Microwave Photonics". In: IEEE Microwave Magazine 16.8 (2015), pp. 28-45. ISSN: 1527-3342. DOI: 10.1109/MMM . 2015. 2442932.

[6] J. S. Kilby. "Invention of the integrated circuit". In: IEEE Transactions on Electron Devices 23.7 (1976), pp. 648-654. ISSN: 0018-9383. DOI: 10. 1109/T-ED.1976.18467.

[7] David Marpaung et al. "Integrated microwave photonics". In: Laser $\mathscr{6}$ Photonics Reviews 7.4 (2013), pp. 506-538. ISSN: 1863-8899.

[8] Kerstin Wörhoff et al. "TriPleX: a versatile dielectric photonic platform". In: Advanced Optical Technologies 4 (2015), pp. 189-207.

[9] Jared F. Bauters et al. "Planar waveguides with less than $0.1 \mathrm{~dB} / \mathrm{m}$ propagation loss fabricated with wafer bonding". In: Opt. Express 19.24 (Nov. 2011), pp. 24090-24101. DOI: 10.1364/OE. 19.024090. 
[10] Javier S. Fandiño et al. "A monolithic integrated photonic microwave filter". In: Nature Photonics 11 (2016).

[11] A. Spott et al. "Heterogeneous Integration for Mid-infrared Silicon Photonics". In: IEEE Journal of Selected Topics in Quantum Electronics 23.6 (2017), pp. 1-10.

[12] COST-European Network for High Performance Integrated Microwave Photonics. 2017. URL: http : / / www . cost . eu / COST _ Actions / ca / CA16220.

[13] Photonic Hybrid Assembly Through Flexible Waveguides. 2014. URL: http: //www . phastflex.eu/.

[14] Naser Hosseini et al. "Stress-optic modulator in TriPleX platform using a piezoelectric lead zirconate titanate (PZT) thin film". In: Opt. Express 23.11 (June 2015), pp. 14018-14026. DOI: 10.1364/0E.23.014018.

[15] V. Urick, J. McKinney, and K. Williams. Fundamentals of Microwave Photonics. Wiley Series in Microwave and Optical Engineering, 2015.

[16] K. J. Williams, L. T. Nichols, and R. D. Esman. "Externally-modulated $3 \mathrm{GHz}$ fibre optic link utilising high current and balanced detection". In: Electronics Letters 33.15 (July 1997), pp. 1327-1328. ISSN: 0013-5194. DOI: $10.1049 / \mathrm{el}: 19970856$.

[17] Xiuyou Han, Xiang Chen, and Jianping Yao. "Simultaneous even- and third-order distortion suppression in a microwave photonic link based on orthogonal polarization modulation, balanced detection, and optical sideband filtering". In: Opt. Express 24.13 (June 2016), pp. 14812-14827. DOI: $10.1364 /$ OE . 24.014812.

[18] C. H. Cox. Analog Optical Links: Theory and Practice. Cambridge University Press, 2004.

[19] David Marpaung. High Dynamic Range Analog Photonic Links - Design E Implementation, PhD thesis, University of Twente, 2009.

[20] H. Al-Raweshidy and S. Komaki. Radio over Fiber Technologies for Mobile Communications Networks. Boston: Artech House, 2002.

[21] John G. Proakis and Dimitris G. Manolakis. Digital Signal Processing (3rd Ed.): Principles, Algorithms, and Applications. Upper Saddle River, NJ, USA: Prentice-Hall, Inc., 1996. ISBN: 0-13-373762-4.

[22] F. Morichetti et al. "The first decade of coupled resonator optical waveguides: bringing slow light to applications". In: Laser 8 Photonics Reviews 6.1 (2011), pp. 74-96.

[23] H. P. Uranus et al. "Pulse advancement and delay in an integratedoptical two-port ring-resonator circuit: direct experimental observations". In: Opt. Lett. 32.17 (2007), pp. 2620-2622. DOI: 10.1364/OL . 32. 002620.

[24] Leimeng Zhuang. Ring Resonaotr-Based Broadband Photonic Beam Former for Phased Array Antennas. PhD thesis, University of Twente, 2010. 
[25] S. R. Saunders and A. Aragón-Zavala. Antennas and Propagation for Wireless Communication Systems, 2nd Edition. John Wiley \& Sons, Inc., 2005.

[26] R. J. Mailloux. Phased Array Antenna Handbook, Second Edition. Artech House Publisher, 2005.

[27] M. Skolnik. Introduction to Radar Systems, 3rd Edition. McGraw-Hill, 2001.

[28] B. Allen et al. Ultra Wideband Antennas and Propagation for Communications, Radar and Imaging. John Wiley \& Sons, Inc, 2006.

[29] R. A. Monzingo, R. L. Haupt, and T. W. Miller. Introduction to Adaptive Arrays, Second Edition. SciTech Publishing, 2011.

[30] R. C. Hansen and Inc John Wiley \& Sons. Phased Array Antennas. 1st ed. Wiley-Interscience, 1998. ISBN: 0471224219 (electronic bk.) URL: http: //dx.doi.org/10.1002/0471224219.

[31] T. Rappaport. Wireless Communications: Principles and Practice, Second Edition. Prentice Hall PTR Upper Saddle River, NJ, USA, 2001.

[32] M. S. Neiman. "The Principle of Reciprocity in Antenna Theory". In: Proceedings of the IRE 31.12 (Dec. 1943), pp. 666-671. ISSN: 0096-8390. DOI: 10.1109/JRPROC. 1943.233683.

[33] Liu Li, Yang Ting, and Dong Jian-Ji. "Microwave photonic filter with a continuously tunable central frequency using an SOI high- Q microdisk resonator". In: Chinese Physics B 23.9 (2014), p. 093201.

[34] X. Zou et al. "Photonic-Assisted Microwave Channelizer With Improved Channel Characteristics Based on Spectrum-Controlled Stimulated Brillouin Scattering". In: IEEE Transactions on Microwave Theory and Techniques 61.9 (Sept. 2013), pp. 3470-3478.

[35] L. Gao, X. Chen, and J. Yao. "Tunable Microwave Photonic Filter With a Narrow and Flat-Top Passband". In: IEEE Microwave and Wireless Components Letters 23.7 (July 2013), pp. 362-364. ISSN: 1531-1309. DOI: 10.1109/LMWC. 2013.2262263.

[36] J. Mora et al. "Photonic microwave tunable single-bandpass filter based on a Mach-Zehnder interferometer". In: Journal of Lightwave Technology 24.7 (July 2006), pp. 2500-2509. ISSN: 0733-8724. DOI: 10 . $1109 /$ JLT . 2006.874652 .

[37] C. K. Madsen and J. H. Zhao. Optical Filter Design and Analysis: A Signal Processing Approach. John Wiley \& Sons, Inc., 2001.

[38] C. G. H. Roeloffzen et al. "Low-Loss $\mathrm{Si}_{3} \mathrm{~N}_{4}$ TriPleX Optical Waveguides: Technology and Applications Overview". In: IEEE Journal of Selected Topics in Quantum Electronics 24.4 (July 2018), pp. 1-21. ISSN: 1077260X. DOI: 10.1109/JSTQE. 2018.2793945. 
[39] Youwen Fan. Semiconductor-glass Waveguide Hybrid Lasers with Ultrahigh Spectral Purity. PhD thesis, University of Twente, 2017.

[40] Y. Fan et al. "290 Hz intrinsic linewidth from an integrated optical chipbased widely tunable $\mathrm{InP}-\mathrm{Si}_{3} \mathrm{~N}_{4}$ hybrid laser". In: 2017 Conference on Lasers and Electro-Optics (CLEO). May 2017, pp. 1-2.

[41] Ruud M. Oldenbeuving. Spectral control of Diode Lasers Using External Waveguide Circuits. PhD thesis, University of Twente, 2013.

[42] M. J. LaGasse and S. Thaniyavaru. "Bias-free high-dynamic-range phasemodulated fiber-optic link". In: IEEE Photonics Technology Letters 9.5 (1997), pp. 681-683. ISSN: 1041-1135. DOI: 10.1109/68.588207.

[43] Leimeng Zhuang et al. "On-chip microwave photonic beamformer circuits operating with phase modulation and direct detection". In: Opt. Express 22.14 (), pp. 17079-17091.

[44] V. J. Urick et al. "Phase Modulation With Interferometric Detection as an Alternative to Intensity Modulation With Direct Detection for AnalogPhotonic Links". In: IEEE Transactions on Microwave Theory and Techniques 55.9 (2007), pp. 1978-1985. ISSN: 0018-9480. DOI: 10.1109/TMTT. 2007.904087.

[45] VPIphotonics software. http://www.vpiphotonics.com.

[46] Promis2Day proposal. https : //www.narcis.nl/research/RecordID/ OND1352190.

[47] A. Meijerink et al. "Novel Ring Resonator-Based Integrated Photonic Beamformer for Broadband Phased Array Receive Antennas;Part I: Design and Performance Analysis". In: Journal of Lightwave Technology 28.1 (2010), pp. 3-18. ISSN: 0733-8724. DOI: 10.1109/JLT .2009. 2029705.

[48] L. Zhuang et al. "Novel Ring Resonator-Based Integrated Photonic Beamformer for Broadband Phased Array Receive Antennas;Part II: Experimental Prototype". In: Journal of Lightwave Technology 28.1 (2010), pp. 19-31. ISSN: 0733-8724. DOI: 10.1109/JLT.2009.2032137.

[49] F. Morichetti et al. "Box-Shaped Dielectric Waveguides: A New Concept in Integrated Optics?" In: Journal of Lightwave Technology 25.9 (Sept. 2007), pp. 2579-2589. ISSN: 0733-8724. DOI: 10.1109/JLT . 2007.903601.

[50] L. Bliek et al. "Automatic Tuning of a Novel Ring Resonator-based Photonic Beamformer for a Transmit Phased Array Antenna". In: ArXiv eprints (Aug. 2018). arXiv: 1808.04814.

[51] Y. Fan et al. "Optically Integrated $\mathrm{InP}-\mathrm{Si}_{3} \mathrm{~N}_{4}$ Hybrid Laser". In: IEEE Photonics Journal 8.6 (Dec. 2016), pp. 1-11. ISSN: 1943-0655. DOI: 10. 1109/JPHOT . 2016.2633402.

[52] EU-Project 3PEAT. http://ict-3peat.eu. 
[53] L. Zhuang et al. "Ring resonator-based on-chip PM-IM convertor for high-performance microwave photonic links". In: 2013 IEEE International Topical Meeting on Microwave Photonics (MWP). Oct. 2013, pp. 123126. DOI: $10.1109 /$ MWP. 2013.6724035. 


\section{Journal Papers}

- Chris G.H. Roeloffzen, Leimeng Zhuang, Caterina Taddei, Arne Leinse, René G. Heideman, Paulus W.L. van Dijk, Ruud M. Oldenbeuving, David A.I. Marpaung, Maurizio Burla, Klaus-J. Boller, "Silicon nitride microwave circuits", Optics Express, vol. 21, no. 19, pp. 22937 - 22961, September 2013.

- Leimeng Zhuang, Caterina Taddei, Marcel Hoekman, Arne Leinse, René G. Heideman, Paulus van Dijk, Chris G.H. Roeloffzen, "Ring resonator-based on-chip modulation transformer for high-performance phase-modulated microwave photonic links", Optics Express, vol. 21, no. 22, pp. 25999 - 26013, November 2013.

- Leimeng Zhuang, Marcel Hoekman, Caterina Taddei, Arne Leinse, René G. Heideman, Adriaan Hulzinga, Jaco Verpoorte, Ruud M. Oldenbeuving, Paulus W.L. van Dijk, Klaus-J. Boller and others, "On-chip microwave photonic beamformer circuits operating with phase modulation and direct detection", Optics Express, vol. 22, no. 14, pp. 17079 17091, July 2014.

- Leimeng Zhuang, Maurizio Burla, Caterina Taddei, Chris G.H. Roeloffzen, Marcel Hoekman, Arne Leinse, Klaus-J. Boller, Arthur J. Lowery, "Integrated microwave photonic splitter with reconfigurable amplitude, phase, and delay offsets", Optics Express, vol. 40, no. 23, pp. 5618 5621, December 2015.

- C Xiong, X Zhang, A Mahendra, J He, D-Y Choi, CJ Chae, D Marpaung, Arne Leinse, RG Heideman, M Hoekman, CGH Roeloffzen, RM Olden- 
beuving, PWL van Dijk, C Taddei, PHW Leong, BJ Eggleton, "Compact and reconfigurable silicon nitride time-bin entanglement circuit", Optica, vol. 2, no. 8, pp. $724-727$, August 2015.

- Yiwei Xie, Zihan Geng, Leimeng Zhuang, Maurizio Burla, Caterina Taddei, Marcel Hoekman, Arne Leinse, Chris G.H. Roeloffzen, Klaus-J. Boller, Arthur J. Lowery, "Programmable optical processor chips: toward photonic RF filters with DSP-level flexibility and MHz-band selectivity", Nanophotonics, vol. 7, no. 2, pp. 421 - 454, October 2017.

- Chris G.H. Roeloffzen, Marcel Hoekman, Edwin J. Klein, Lennart S. Wevers, Roelof Bernardus Timens, Denys Marchenko, Dimitri Geskus, Ronald Dekker, Andrea Alippi, Robert Grootjans, Albert van Rees, Ruud M. Oldenbeuving, Jörn P. Epping, René G. Heideman, Kerstin Wörhoff, Arne Leinse, Douwe Geuzebroek, Erik Schreuder, Paulus W.L. van Dijk, Ilka Visscher, Caterina Taddei, Youwen Fan, Caterina Taballione, Yang Liu, David Marpaung, Leimeng Zhuang, Meryem Benelajla, Klaus-J. Boller, "Low-loss Si3N4 TriPleX optical waveguides: Technology and applications overview", IEEE journal of selected topics in quantum electronics, vol.24, no. 4, pp. 1 - 21, July 2018.

- Laurens Bliek, Sander Wahls, Ilka Visscher, Caterina Taddei, Roelof Bernardus Timens, Ruud Oldenbeuving, Chris G.H. Roeloffzen, M. Verhaegen, "Automatic Tuning of a Novel Ring Resonator-based Photonic Beamformer for a Transmit Phased Array Antenna", arXiv preprint arXiv:1808.04814, August 2018.

- Caterina Taddei, Leimeng Zhuang, Chris G.H. Roeloffzen, Marcel Hoekman, Klaus-J. Boller, "Very-high-selectivity on-chip optical bandpass filter with sub-100 MHz flat-top and under-2 shape factor", submitted to IEEE Photonics Technology Letters, October 2018.

\section{Conference Papers}

- Leimeng Zhuang, Caterina Taddei, Marcel Hoekman, Arne Leinse, René G. Heideman, Paul van Dijk, Chris G.H. Roeloffzen, "Ring resonator-based on-chip PM-IM convertor for high-performance microwave photonic links", International Topical Meeting on Microwave Photonics (MWP), Washington D.C., USA, October 2013, pp. 123 126.

- Caterina Taddei, Nguyen T.H. Yen, Leimeng Zhuang, Marcel Hoekman, Arne Leinse, René G. Heideman, Paul van Dijk, Chris G.H. Roeloffzen, "Waveguide filter-based on-chip differentiator for microwave photonic signal processing", International Topical Meeting on Microwave 
Photonics (MWP), Washington D.C., USA, October 2013, pp. 210 213.

- Leimeng Zhuang, Caterina Taddei, Marcel Hoekman, Ruud Oldenbeuving, Klaus-J. Boller, Chris G.H. Roeloffzen, "Recent Progress in Silicon Nitride Waveguide-based Integrated Microwave Photonics", Progress in Electromagnetics Research Symposium (PIERS), Guangzhou, China, September 2014.

- Caterina Taddei, Leimeng Zhuang, Marcel Hoekman, Arne Leinse, Ruud Oldenbeuving, Paul van Dijk, Chris G.H. Roeloffzen, "Fully reconfigurable coupled ring resonator-based bandpass filter for microwave signal processing", International Topical Meeting on Microwave Photonics (MWP) and the 2014 9th Asia-Pacific Microwave Photonics Conference (APMP), Sapporo, Japan, October 2014, pp.44 - 47.

- Caterina Taddei, Leimeng Zhuang, Marcel Hoekman, Chris G.H. Roeloffzen, Klaus-J. Boller, "Reconfigurable coupled ring resonator waveguide-based bandpass filter for signal processing", The European Conference on Lasers and Electro-Optics, Munich, Germany, June 2015, pp. CI_4_5.

- Chris G.H. Roeloffzen, Ruud M. Oldenbeuving, Roelof Bernardus Timens, Paulus van Dijk, Caterina Taddei, Arne Leinse, Marcel Hoekman, René G. Heideman, Leimeng Zhuang, David Marpaung, "Integrated optical beamformers", Optical Fiber Communication Conference, Los Angeles, California, March 2015, pp. Tu3F - 4.

- Caterina Taddei, Marcel Hoekman, Chris G.H. Roeloffzen, Klaus-J. Boller, "Design of Zero-Dispersion Optical Lattice Filters for Wavelength Division Multiplexing Systems", Symposium IOP Photonic Devices, Amsterdam, The Netherlands, 2015.

- Caterina Taddei, Chris G.H. Roeloffzen, Marcel Hoekman, Arne Leinse, Klaus-J. Boller, "Integrated microwave photonics filters", Physics@FOM, Veldhoven, The Netherlands, 2016.

- Chris Roeloffzen, Paul van Dijk, Ruud Oldenbeuving, Caterina Taddei, Dimitri Geskus, Ilka Dove, Roelof Bernardus Timens, Jörn Epping, Arne Leinse, René Heideman, "Enhanced coverage though optical beamforming in fiber wireless networks", International Conference on Transparent Optical Networks (ICTON), 2017 19th, Girona, Spain, July 2017, pp. 1 -4 .

- Caterina Taddei, "Hybrid Integrated Beamforming Network", Appplied NanoPhotonics Meeting, Enschede, The Netherlands, 2017.

- Chris Roeloffzen, Ilka Visscher, Caterina Taddei, Dimitri Geskus, Ruud Oldenbeuving, Jörn Epping, Roelof Bernardus Timens, Paul van Dijk, 
René Heideman, Marcel Hoekman, Robert Grootjans, Laurens Bliek, Sander Wahls, Michel Verhaegen, "Integrated microwave photonics for 5G", CLEO: Applications and Technology, San Jose, California, May 2018, pp. JTh3D - 2.

- Caterina Taddei, Chris Roeloffzen, Ilka Visscher, Marcel Hoekman, Lennart Wevers, Robert Grootjans, Paul Kapteijn, Dimitri Geskus, Andrea Alippi, Ronald Dekker, Ruud Oldenbeuving, Jörn Epping, Roelof Bernardus Timens, Edwin Klein, Arne Leinse, Paul Van Dijk, René Heideman, "Hybrid Integrated Microwave Photonic Broadband Beamformers for Ka-Band Array Antennas", International Topical Meeting on Microwave Photonics, Toulouse, France, October 2018. 


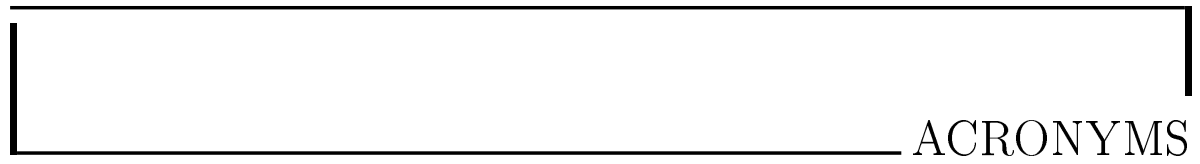

AE antenna element.

AMZI asymmetric Mach-Zehnder interferometer.

APL analog photonic link.

CDR compression dynamic range.

CROW coupled resonator optical waveguide.

CW continuous wave.

DBR distributed Bragg reflector.

DFB distributed-feedback.

DSB double-sideband.

DTFT discrete-time Fourier transform.

FIR finite impulse response.

FSR free spectral range.

FWH full width at half maximum.

HD harmonic distorsion.

IF intermediate frequency.

IIP input intercept point. 
IIP3 input intercept point of the third order.

IIR infinite impulse response.

IMD intermodulation distorsion.

IMD3 third-order intermodulation distorsion.

IMWP integrated microwave photonics.

InP Indium Phosphide.

MWP microwave photonics.

MZI Mach-Zehnder interferometer.

MZM Mach-Zehnder modulator.

OBFN optical beam-forming network.

OIP output intercept point.

OIP3 output intercept point of the third order.

OSBF optical sideband filter.

PD photodetector.

PLCs planar light wave circuits.

PSD power spectral density.

$\mathbf{R F}$ radio frequency.

RIN relative intensity noise.

$\mathbf{R R}$ ring resonator.

SC suppressed-carrier.

SDL switched delay lines.

SF shape factor.

SFDR spurious-free dynamic range.

SNR signal-to-noise ratio.

SSB single-sideband.

SSB-FC single-sideband full-carrier. 
SSB-SC single-sideband suppresed-carrier.

TTD true-time delay.

VCSELs vertical-cavity surface-emitting lasers.

VNA vector network analyzer. 
Italia, Perugia, Tuoro sul Trasimeno.

This is the place where, sitting and deeply breathing, every action is absorbed and every big decision in my life is taken, and so do I, now. Sitting here, writing this very last part of my thesis, absorbing every single bit of this past 5 years and something.

"A very long time for JUST a thesis", someone might say.

"I agree, but totally worthwhile", I would answer.

February 2013, extremely cold for my taste, but $t j a^{1} \ldots$

The first smile I meet in the corridor of the University of Twente: Chris. I am deeply thankful and proud that you have chosen me among many. Thanks for this great opportunity that has changed my prospect. Thanks for the support, tecnical and moral, especially during the tough time of the writing of this thesis. Thanks for the long discussions to make sure I would understand things for ever, don't worry, one day or another the light will burn! Thanks for having faith in me, it is so important to me. Last but not least thanks for the awesome dish antenna you gave me for watching my beloved Italian soccer! There would be many other reasons, but I have to thank many others and I am sure I will have still chances to thank you many more times!

With the same depth, I thank Klaus, my supervisor, my mentor, the person who had to deal with Caterina, better known as stubborn engineer. Thanks for supporting me in this not very easy journey. You, with your calm, your love for science and for clarity, your politeness and perseverance made me a better person, not only as a researcher, but also in a broader sense, and I will treasure

\footnotetext{
${ }^{1}$ typical dutch exclamation of resignation and uncertainty, you have to say such word shrugging your shoulder, otherwise the effect is not as stressed as it should be!
} 
it, always. A special, enormous gratitude towards you for the time you spent correcting my thesis.

The TE group, my first acquired family. First, I would deeply thank Lilian, you are such a lovely woman, always ready to help, to listen, to support, you are precious! Thanks for everything you have done for me. Leimeng, dude, you have been the best guide I could ever expect to have, a mix of explosive fun and pure science. Competence, patience and generosity, all in one, there is nothing more I could have asked for! Arjan, without you the first months could have been so difficult, but you, with your kindness, helped me to feel home, thanks! Stefan, Robert, Ibrahim, Olga, thanks guys for the laughs, for the shared multi-ethnic lunches, for the serious discussions and for sharing with me part of your paths. Frits, thanks for the nice gifts, and the chats at the coffee corner. Alex, my friend, my supporter, my paranymph. You have been the good friend that is so rare to find, and I was so lucky to find you just there, in the office! I have so many nice memories with you and I keep them for ever with me. Thanks for being the way you are.

The LPNO group, my second acquired family (just considering the time line!). It was not an easy time, to change group in the middle of my $\mathrm{PhD}$. However, you welcomed me with such spontaneity, that I did not get lost. Thanks to all of you, Peter, Bert, for the useful technical discussions as well as the food and wine related discussions! Thanks Carla for taking care of my last steps towards the big day! I have to thank my office mates, Yin, Casper, Jonathan, and Jesse, since one day I simply popped up in the office and you guys have been so nice in welcoming me that the transition from TE group to LPNO group was unexpectedly easy and smooth. Youwen, thanks for always kindly answer to my existential doubts and sharing your expertise. Marco and Eliana, it was nice to share a couple of years with you, the climbing, the rock metal gothic music at the Loch, the evenings at the Rocks, I wish you the best in Valencia! Caterina, ma che te devo di! I am extremely happy that one day you came in and you started cheering me up in this last part of the Great Adventure, also know as PhD! I can tell you, it seems to be a never ending story, but, well, at the top there is such a view!

The hard core group. Aga, Matteo, Marco, Riccardo, Giuseppe, Gerard, Niloofar, Giorgio, Virginia. I have uncountable memories, so many adventures, evenings that were supposed to be calm and somehow ended up at 6 in the morning, endless time spent on cooking like if we were just one big family. A special thanks to my friend, Virginia. Thanks for teaching me that one of the bet "spot" where to sit is the unstable equilibrium point.

The life after $\mathrm{PhD}$. There is a life indeed. I have to thank all the people of LioniX International, especially Hans and René, who did not think twice about giving me the chance to be part of LioniX. I would like to thank especially the people of the "karakterisatie" team, Ruud, Jörn, Paul, Robert. Thanks for the chats, for the advices, for the knowledge sharing, for the support, it is a 
great privilege to work with so many talented people. Ilka, a special thanks to you. You are incredibly strong, motivated, tireless and committed colleague. Thanks for being always willing to help, support me and to share your time with me. It meant a lot.

The friends for ever. Giulia, Giacomo, Fabrizio, Mattia, thanks for being there, always, unconditionally.

A special thank to all the committee members, who decided to accept the invitation to the $\mathrm{PhD}$ defence and invested time in reading my thesis.

My family. Mamma, babbo, Francesca, Zio Riccardo, Giulia, Andrea, Catiuscia, nonna Amabilia, you are my strength. You have always let me free to decide and to even make mistakes, to grow, and to become the person I am. A profound thanks, without you I could not be here. My gratitude goes also to my parents in law, Mara and Alessandro, who welcomed me as a daughter.

Federico and Ettore, my everything. Only a "thank you" is not enough. Federico, you have been necessary as air, a strong support, a delicate push, pure joy. My deepest gratitude to you. You have shown me a world of kindness and respect. Ettore, pure love. I do not even recall how was life without that super smile!

November 2018, warm autumnal sunny day. It is almost time for the big day, and with a little help from all of you it will definitely be a big day! 


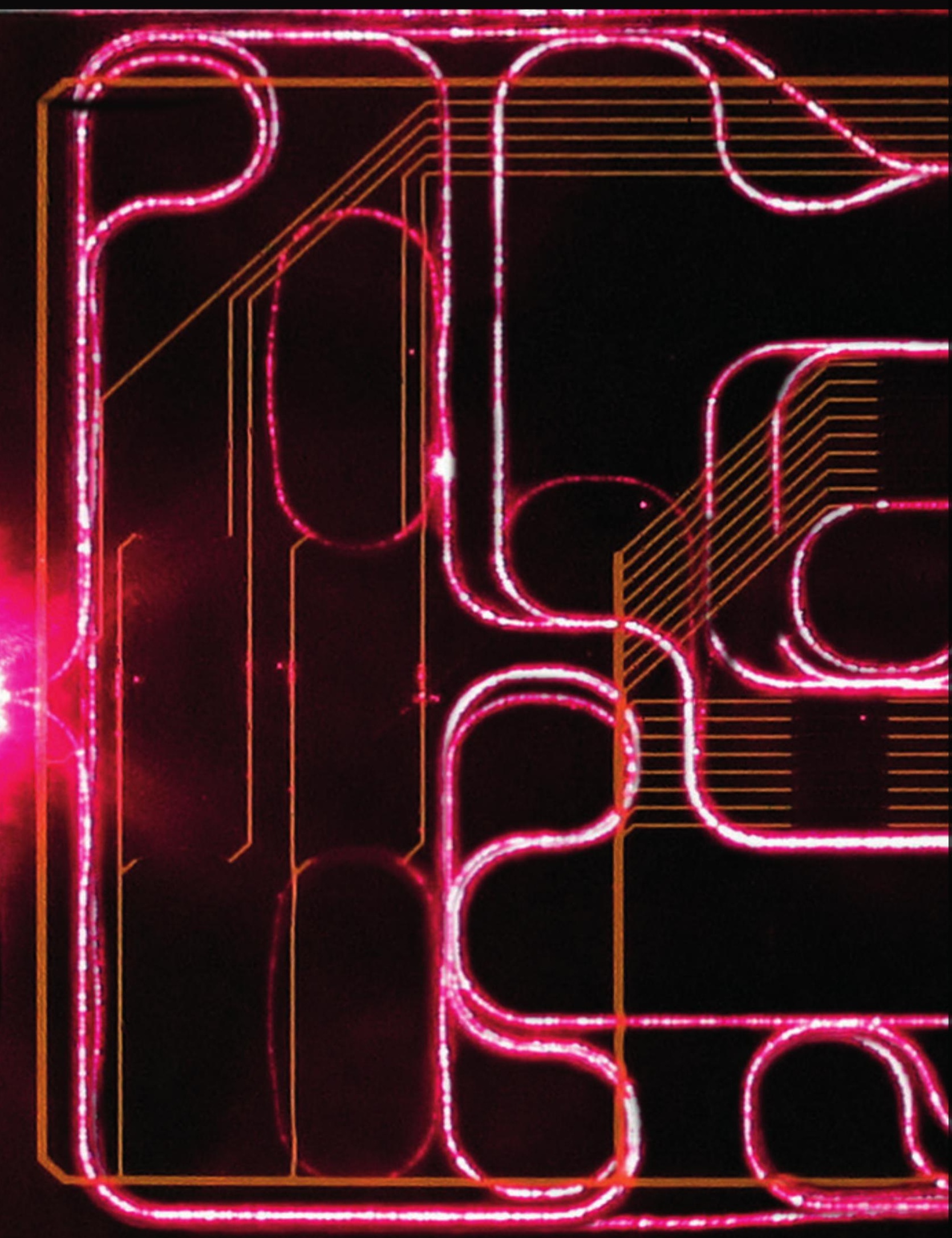

